\title{
Multi-Component Bifunctionalization of Methyl Ketones Enabled by Heterogeneous Catalysis and Solar Photocatalysis in Water
}

\author{
Jun $\mathrm{Xu},{ }^{\dagger}$ Lei He,${ }^{\dagger}$ Chenfeng Liang, ${ }^{\dagger}$ Xiaoguang Yue,${ }^{\dagger}$ Yani Ouyang, ${ }^{\dagger}$ and Pengfei Zhang ${ }^{\dagger}, *$ \\ $\dagger$ College of Material, Chemistry and Chemical Engineering, Key Laboratory of Organosilicon Chemistry and \\ Material Technology, Ministry of Education, Hangzhou Normal University, Hangzhou, 311121, China. \\ *Email: pfzhang@hznu.edu.cn
}

Number of pages: 80

Number of figures: 115

Number of tables: 6 


\section{Table of contents}

General Information

$\begin{array}{ll}\text { 1. Experimental Section } & \mathrm{S} 2\end{array}$

2. Characterization of the Products $\quad$ S9

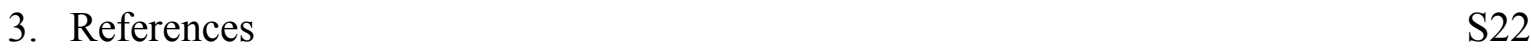

4. Copies of NMR Spectra $\quad$ S23

5. Copies of HRMS Spectra S63 


\section{General Information}

All reagents and deuterated solvents were commercially available and used without further purification. All products were separated by silica gel (200-300 mesh) column chromatography with petroleum ether $(\mathrm{PE})\left(60-90{ }^{\circ} \mathrm{C}\right)$ and ethyl acetate $(\mathrm{EA}) .{ }^{1} \mathrm{H},{ }^{13} \mathrm{C}$ and ${ }^{19} \mathrm{~F}$ NMR spectra were recorded on Bruker Advance 500 spectrometer at ambient temperature with $\mathrm{CDCl}_{3}$ or $\mathrm{CD}_{3} \mathrm{SOCD}_{3}$ as solvent and tetramethylsilane (TMS) as the internal standard. Melting points were determined on an X-5 Data microscopic melting point apparatus. Analytical thin layer chromatography (TLC) was performed on Merk precoated TLC (silica gel 60 F254) plates. Compounds for HRMS were analyzed by positive mode electrospray ionization (ESI) using Agilent 6530 QTOF.

\section{Experimental Section}

\subsection{General procedure for the performance of multi-component reaction}

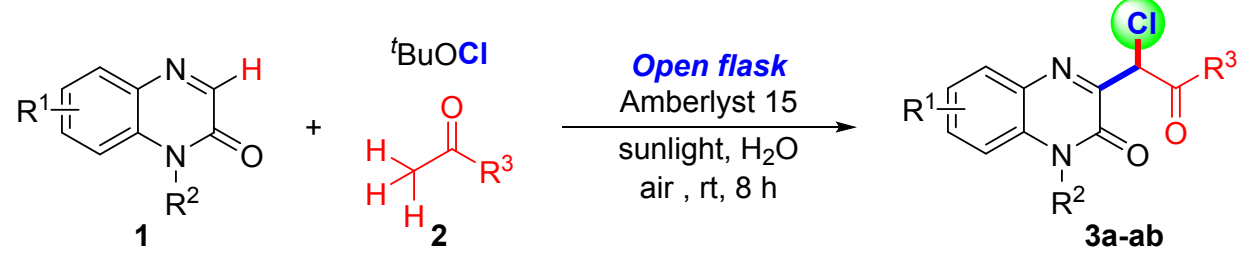

To a $15 \mathrm{~mL}$ tube was added quinoxalinones (1) $(0.2 \mathrm{mmol})$, methyl ketones (2) (0.6 mmol), Amberlyst $15(50 \mathrm{mg})$ and $\mathrm{H}_{2} \mathrm{O}(3 \mathrm{~mL}) .0 .24 \mathrm{mmol}$ of ${ }^{t} \mathrm{BuOCl}$ (as batches of $0.06 \mathrm{mmol}$ each with an interval of $2.0 \mathrm{~h}$ ) was then added to the above mixture under vigorous stirring with the irradiation of sunlight for 8 hours. After the completion (as indicated by TLC), the resulting aqueous phase was extracted with ethyl acetate and the collected organic layer was washed with brine, dried with $\mathrm{MgSO}_{4}$. The solvent was removed under reduced pressure, and the crude product was further purified by silica gel column chromatography (200-300 mesh silica gel, $\mathrm{PE}(v) / \mathrm{EA}(v)=3: 1,200 \mathrm{~mL})$ to afford the target product. Of note, during the purification of products, the eluent was recycled through vacuum distillation and was reused for further purification.

\subsection{General procedure for the gram-scale synthesis of compound 3a}

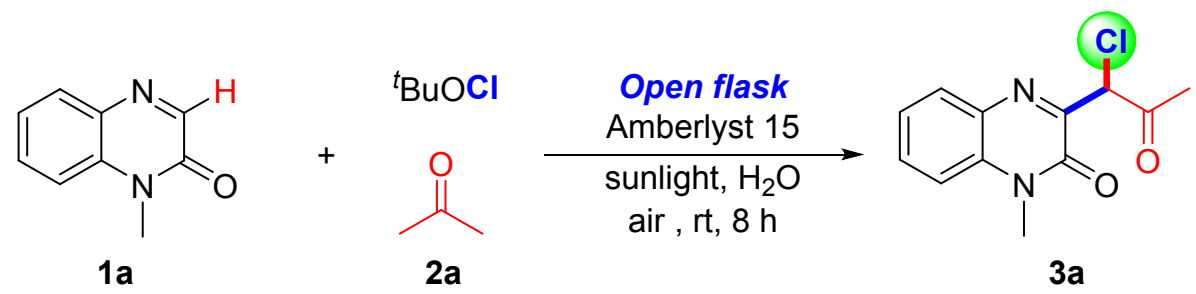

To a $250 \mathrm{~mL}$ flask was added quinoxalinone (1a) $(8.0 \mathrm{mmol})$, acetone (2a) $(24.0 \mathrm{mmol})$, Amberlyst $15(2.0 \mathrm{~g})$ and $\mathrm{H}_{2} \mathrm{O}(120 \mathrm{~mL}) .9 .6 \mathrm{mmol}$ of ${ }^{t} \mathrm{BuOCl}$ (as batches of $2.4 \mathrm{mmol}$ each with an interval of $2.0 \mathrm{~h}$ ) was then added to the above mixture under vigorous stirring with the irradiation of sunlight for 8 hours. After the completion (as indicated by TLC), the resulting aqueous phase was extracted with ethyl acetate and the collected organic layer was washed with brine, dried with $\mathrm{MgSO}_{4}$. The solvent was removed under reduced pressure, and the crude 
product was further purified by silica gel column chromatography (200-300 mesh silica gel, $\mathrm{PE}(v) / \mathrm{EA}(v)=3: 1,800 \mathrm{~mL})$ to afford the target product. Of note, during the purification of products, the eluent was recycled through vacuum distillation and was reused for further purification.

\subsection{General procedure for the synthesis of 4a using 3a as starting material}

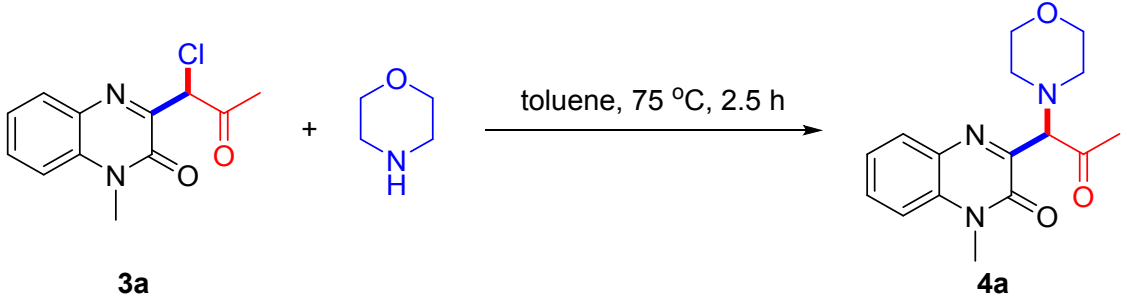

A mixture of compound 3a $(0.2 \mathrm{mmol})$, morpholine (1.5 equiv.), and toluene $(2.0 \mathrm{~mL})$ in a 15 $\mathrm{mL}$ tube was stirred at $75{ }^{\circ} \mathrm{C}$ for 2.5 hours. After the completion (as indicated by TLC), the reaction was quenched with saturated $\mathrm{NaHCO}_{3}$. The mixture was then extracted with ethyl acetate and the collected organic layer was washed with brine, dried with $\mathrm{MgSO}_{4}$. The solvent was removed under reduced pressure, and the crude product was further purified by silica gel column chromatography (200-300 mesh silica gel, $\operatorname{PE}(v) / \mathrm{EA}(v)=4: 1,200 \mathrm{~mL})$ to afford the target product 4a. Of note, during the purification of products, the eluent was recycled through vacuum distillation and was reused for further purification.

\subsection{General procedure for the synthesis of $4 \mathrm{~b}$ using $3 \mathrm{a}$ as starting material}<smiles>CC(=O)C(Cl)c1nc2ccccc2n([13CH3])c1=O</smiles><smiles>CCOP(=O)(OCC)OCC</smiles>

toluene, $120^{\circ} \mathrm{C}, 4 \mathrm{~h}$<smiles>CCOP(=O)(OCC)C(C(C)=O)c1nc2ccccc2n(C(C)(C)C)c1=O</smiles>

A mixture of compound $3 \mathrm{a}(0.2 \mathrm{mmol})$, triethyl phosphite $(1.5$ equiv. $)$, and toluene $(2.0 \mathrm{~mL})$ in a $15 \mathrm{~mL}$ tube was stirred at $120{ }^{\circ} \mathrm{C}$ for 4 hours. After the completion (as indicated by TLC), the reaction was quenched with saturated $\mathrm{NaHCO}_{3}$. The mixture was then extracted with ethyl acetate and the collected organic layer was washed with brine, dried with $\mathrm{MgSO}_{4}$. The solvent was removed under reduced pressure, and the crude product was further purified by silica gel column chromatography (200-300 mesh silica gel, $\operatorname{PE}(v) / \mathrm{EA}(v)=4: 1,200 \mathrm{~mL})$ to afford the target product $\mathbf{4 b}$. Of note, during the purification of products, the eluent was recycled through vacuum distillation and was reused for further purification.

\subsection{General procedure for the synthesis of $4 \mathrm{c}$ using 3a as starting material}

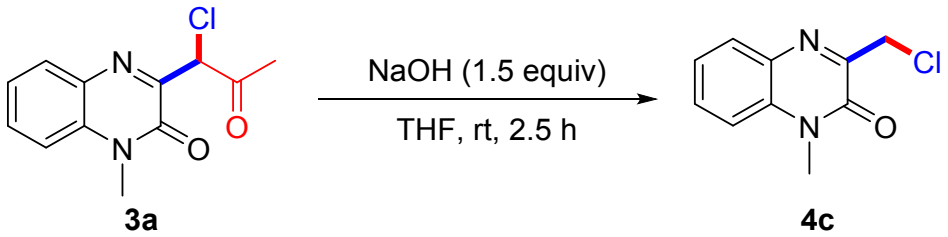


A mixture of compound $3 \mathbf{a}(0.2 \mathrm{mmol}), \mathrm{NaOH}$ (1.5 equiv.), and THF (2.0 mL) in a $15 \mathrm{~mL}$ tube was stirred at room temperature for 2.5 hours. After the completion (as indicated by TLC), the reaction was quenched with saturated $\mathrm{NaHCO}_{3}$. The mixture was then extracted with ethyl acetate and the collected organic layer was washed with brine, dried with $\mathrm{MgSO}_{4}$. The solvent was removed under reduced pressure, and the crude product was further purified by silica gel column chromatography (200-300 mesh silica gel, $\mathrm{PE}(v) / \mathrm{EA}(v)=4: 1,200 \mathrm{~mL})$ to afford the target product 4c. Of note, during the purification of products, the eluent was recycled through vacuum distillation and was reused for further purification.

\subsection{General procedure for the synthesis of $4 \mathrm{~d}$ using $3 \mathrm{a}$ as starting material}

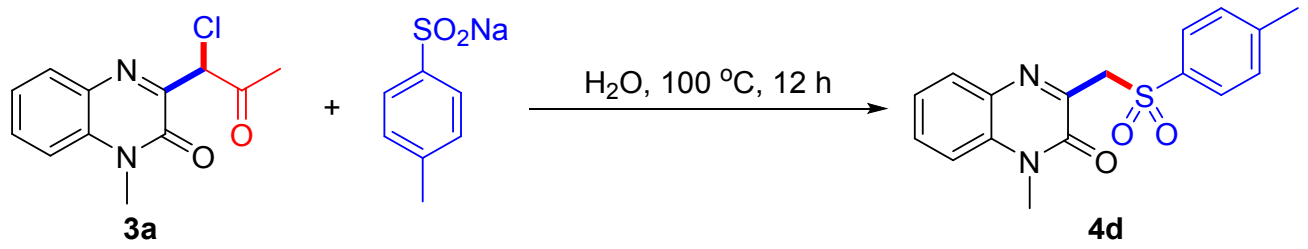

A mixture of compound 3a $(0.2 \mathrm{mmol})$, sodium $p$-toluenesulfinate (1.5 equiv.), and $\mathrm{H}_{2} \mathrm{O}(2.0$ $\mathrm{mL}$ ) in a $15 \mathrm{~mL}$ tube was stirred at $100{ }^{\circ} \mathrm{C}$ for 12 hours. After the completion (as indicated by TLC), the reaction was quenched with saturated $\mathrm{NaHCO}_{3}$. The mixture was then extracted with ethyl acetate and the collected organic layer was washed with brine, dried with $\mathrm{MgSO}_{4}$. The solvent was removed under reduced pressure, and the crude product was further purified by silica gel column chromatography (200-300 mesh silica gel, $\mathrm{PE}(v) / \mathrm{EA}(v)=4: 1,200 \mathrm{~mL})$ to afford the target product 4d. Of note, during the purification of products, the eluent was recycled through vacuum distillation and was reused for further purification.

\subsection{Testing of the recyclability of catalytic system.}

To a $15 \mathrm{~mL}$ tube was added quinoxalinone (1a) $(0.2 \mathrm{mmol})$, acetone (2a) $(0.6 \mathrm{mmol})$, Amberlyst $15(50 \mathrm{mg})$ and $\mathrm{H}_{2} \mathrm{O}(3 \mathrm{~mL}) .0 .24 \mathrm{mmol}$ of ${ }^{t} \mathrm{BuOCl}$ (as batches of $0.06 \mathrm{mmol}$ each with an interval of $2.0 \mathrm{~h}$ ) was then added to the above mixture under vigorous stirring with the irradiation of sunlight for 8 hours. After the completion (as indicated by TLC), the resulting aqueous phase was extracted with ethyl acetate and the collected organic layer was washed with brine, dried with $\mathrm{MgSO}_{4}$. The solvent was removed under reduced pressure, and the crude product was further purified by silica gel column chromatography $(200-300$ mesh silica gel, PE/EA = 3:1) to afford the target product. The resulting aqueous solution containing Amberlyst 15 catalyst was reutilized to catalyze the transformation by directly adding starting materials.

\subsection{The regeneration of Amberlyst 15 catalyst}

After it was recycled for four times, Amberlyst 15 catalyst was first washed with ethanol, ethyl acetate and 1,2-dichloromethane continuously to remove the organic impurities. Then, the catalyst was washed with $\mathrm{HCl}(2 \mathrm{M})$, distilled water and $\mathrm{NaOH}(2 \mathrm{M})$ for three times to remove the water-soluble impurities. After that, the catalyst was immersed into $\mathrm{HCl}(2 \mathrm{M})$ for $12 \mathrm{~h}$, and the resulting catalyst was washed with distilled water repeatedly until the $\mathrm{pH}$ value is about 7 . Finally, it was dried under vacuum condition to give the regenerated Amberlyst 15 catalyst. 
Table S1. Ineffective substrates for the multi-component bifunctionalization of methyl ketones<smiles>O=C1CC(=O)N(Cl)C1=O</smiles><smiles>O=C1CCC(=O)N1Br</smiles><smiles>O=C1CCC(=O)N1I</smiles><smiles>O=c1cnc2ccccc2[nH]1</smiles><smiles>O=c1cnc2ccccc2n1-c1ccccn1</smiles><smiles>c1ccc2ncccc2c1</smiles><smiles>c1ccc2cnccc2c1</smiles><smiles></smiles>

1 ba $1 \mathrm{bb}$ 1 bc $1 \mathrm{bd}$<smiles>CC(=O)CC(C)=O</smiles>

2ba<smiles>CCOC(=O)CC(C)=O</smiles>

2bb<smiles>CCOC(C)=O</smiles>

2bc

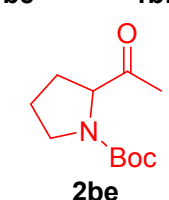

Table S2. Optimization of solvents ${ }^{a}$

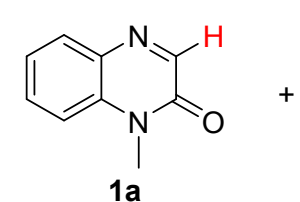

Open flask

${ }^{t} \mathrm{BuOCl}$

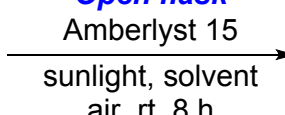
air, rt, $8 \mathrm{~h}$

$2 \mathrm{a}$<smiles>CC(=O)C(Cl)(Cl)c1nc2ccccc2n(C)c1=O</smiles>

$3 a$

\begin{tabular}{ccc}
\hline entry & solvent & yield $(\%)^{b}$ \\
\hline 1 & $\mathrm{H}_{2} \mathrm{O}$ & 78 \\
2 & $\mathrm{PEG} \mathrm{200}$ & trace \\
3 & EtOH & 19 \\
4 & $\mathrm{MeOH}$ & 15 \\
5 & acetone & 85 \\
6 & MeCN & 83 \\
\hline
\end{tabular}

${ }^{a}$ Reaction conditions: 1a (0.2 mmol), 2 a (3.0 equiv.), Amberlyst $15(50 \mathrm{mg}),{ }^{t} \mathrm{BuOCl}$ (1.2 equiv.), solvent $(3.0 \mathrm{~mL})$, sunlight, open flask, air, room temperature, $8 \mathrm{~h} .{ }^{\mathrm{b}}$ Isolated yields.

Table S3. The formation of compounds 7 and 8

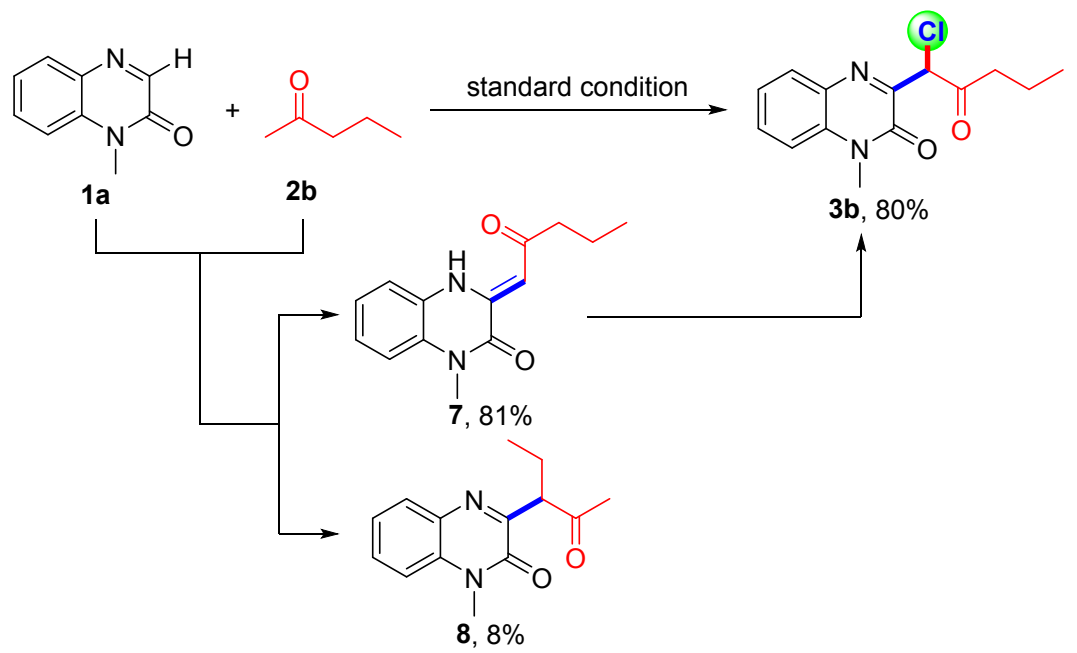


Table S4. Calculation of green chemistry metrics

\begin{tabular}{|c|c|c|c|c|c|c|c|}
\hline Reagent 1 & Reagent 2 & Reagent 3 & Reagent 4 & Reagent 5 & Solvent & Yield & Product \\
\hline $\begin{array}{c}\text { 1a, } 0.2 \\
\text { mmol, } \\
\text { MW: } \\
160.2 \text {, } \\
32.0 \mathrm{mg}\end{array}$ & $\begin{array}{c}\mathbf{2 a}, 0.6 \\
\text { mmol, } \\
\text { MW: } 58.1, \\
34.9 \mathrm{mg}\end{array}$ & $\begin{array}{c}{ }^{t} \mathrm{BuOCl} \\
0.24 \\
\mathrm{mmol}, \\
\mathrm{MW}: \\
108.6 \\
26.1 \mathrm{mg}\end{array}$ & $\begin{array}{c}\text { Amberlyst } \\
15,50.0 \\
\mathrm{mg}\end{array}$ & $\begin{array}{c}\mathrm{O}_{2}, \mathrm{MW}: \\
32.0\end{array}$ & $\begin{array}{l}\text { Water, } \\
3.0 \mathrm{~mL}\end{array}$ & $80 \%$ & $\begin{array}{c}3,0.16 \\
\text { mmol, } \\
\text { MW: } \\
250.7 \text {, } \\
40.1 \\
\text { mg }\end{array}$ \\
\hline
\end{tabular}

$$
\begin{gathered}
\text { Atom Economy }=\frac{\text { MW of desired product }}{\sum \text { of MWof stoichiometric reactants }} \times 100 \% \\
E-\text { Factor }=\frac{\sum \text { of mass of inputs }- \text { mass yield of desired product }}{\text { mass yield of desired product }} \\
\text { Atom Economy }=\frac{250.7}{160.2+58.1+108.6+32.0} \times 100 \%=69.9 \%
\end{gathered}
$$

With catalytic system recycling

$$
E-\text { factor }=\frac{\sum 32.0+34.9+26.1-40.1}{40.1}=1.3
$$

Without catalytic system recycling

$$
E-\text { factor }=\frac{\sum 32.0+34.9+26.1+50.0-40.1}{40.1}=2.6
$$

Table S5. UV-vis absorption spectroscopy of $\mathbf{1 a}$

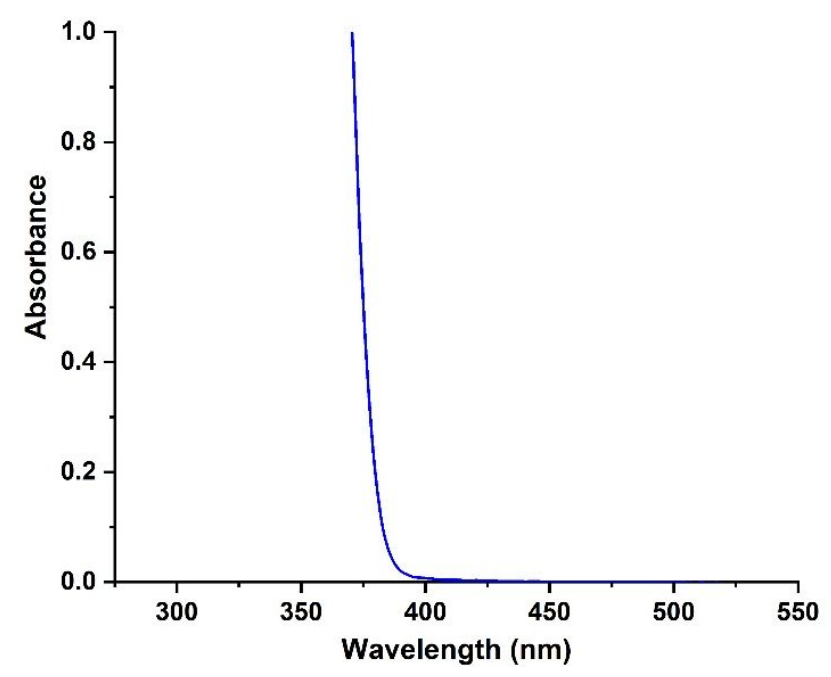


Note: the sample was prepared by mixing compounds $1 \mathbf{a}$ with acetone. The reaction mixture was stirred for $10 \mathrm{~min}$, and the reaction mixture was filtered with a filter. The resulted solution was stored in a light path quartz fluorescence cuvette, and UV-vis absorption spectrum was recorded. 
Table S6. ESR spectra of ${ }^{1} \mathrm{O}_{2}$ triggerred by $1 \mathrm{a}$

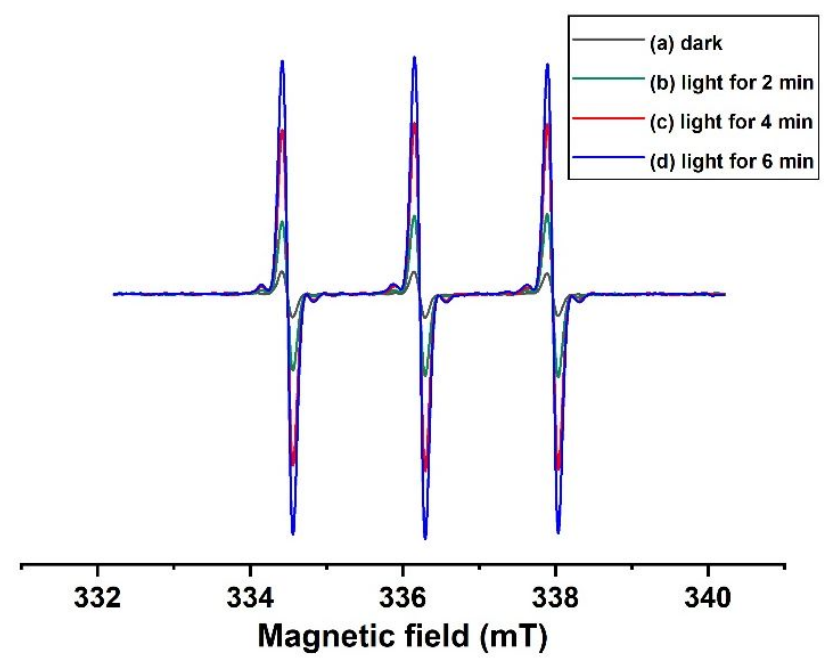

Note: a mixture of 1a and TEMP in acetone was transferred in a capillary tube, then the capillary tube was transferred to a ESR sample tube, the ESR signal was recorded in dark (a); then the tube was irradiated under sunlight, the spectrum was recorded in $2 \mathrm{~min}(\mathbf{b}), 4 \mathrm{~min}(\mathbf{c})$, and 6 min (c) separately. 


\section{Characterization of the Products}

\section{3-(1-Chloro-2-oxopropyl)-1-methylquinoxalin-2(1H)-one (3a)}<smiles>CC(=O)C(Cl)(Cl)c1nc2ccccc2n(C)c1=O</smiles>

Purified by column chromatography using the mixture of petroleum ether and ethyl acetate $(\mathrm{PE} / \mathrm{EA}=3: 1)$ as eluant, white solid $(39 \mathrm{mg}, 78 \%$ yield $)$, m.p. $159-160{ }^{\circ} \mathrm{C} .{ }^{1} \mathrm{H}$ NMR $(500 \mathrm{MHz}$, $\left.\mathrm{CDCl}_{3}\right) \delta 7.91(\mathrm{dd}, J=8.0,1.3 \mathrm{~Hz}, 1 \mathrm{H}), 7.63(\mathrm{ddd}, J=8.6,7.5,1.5 \mathrm{~Hz}, 1 \mathrm{H}), 7.42-7.38(\mathrm{~m}$, $1 \mathrm{H}), 7.35(\mathrm{~d}, J=8.4 \mathrm{~Hz}, 1 \mathrm{H}), 5.68(\mathrm{~s}, 1 \mathrm{H}), 3.72(\mathrm{~s}, 3 \mathrm{H}), 2.56(\mathrm{~s}, 3 \mathrm{H}) ;{ }^{13} \mathrm{C}$ NMR $(126 \mathrm{MHz}$, $\left.\mathrm{CDCl}_{3}\right) \delta 200.2,154.3,153.3,133.7,132.3,131.7,130.8,124.2,113.9,62.3,29.3,27.8$; HRMS (ESI) $\mathrm{m} / \mathrm{z}$ : $[\mathrm{M}+\mathrm{Na}]^{+}$Calcd for $\mathrm{C}_{12} \mathrm{H}_{11} \mathrm{ClN}_{2} \mathrm{O}_{2} \mathrm{Na} 273.0401$; Found 273.0399.

\section{3-(1-Chloro-2-oxopentyl)-1-methylquinoxalin-2(1H)-one (3b)}<smiles>CCCC(=O)C(C)(Cl)c1nc2ccccc2n(C)c1=O</smiles>

Purified by column chromatography using the mixture of petroleum ether and ethyl acetate $(\mathrm{PE} / \mathrm{EA}=3: 1)$ as eluant, white solid (43 mg, 77\% yield), m.p. $153-154{ }^{\circ} \mathrm{C} .{ }^{1} \mathrm{H}$ NMR $(500 \mathrm{MHz}$, $\left.\mathrm{CDCl}_{3}\right) \delta 7.90(\mathrm{dd}, J=8.0,1.0 \mathrm{~Hz}, 1 \mathrm{H}), 7.66-7.58(\mathrm{~m}, 1 \mathrm{H}), 7.39(\mathrm{t}, J=7.3 \mathrm{~Hz}, 1 \mathrm{H}), 7.34(\mathrm{~d}, J$ $=8.4 \mathrm{~Hz}, 1 \mathrm{H}), 5.71(\mathrm{~s}, 1 \mathrm{H}), 3.71(\mathrm{~s}, 3 \mathrm{H}), 2.94(\mathrm{dt}, J=17.8,7.3 \mathrm{~Hz}, 1 \mathrm{H}), 2.79(\mathrm{dt}, J=17.8,7.2$ $\mathrm{Hz}, 1 \mathrm{H}), 1.77-1.69(\mathrm{~m}, 2 \mathrm{H}), 0.98(\mathrm{t}, J=7.4 \mathrm{~Hz}, 3 \mathrm{H}) ;{ }^{13} \mathrm{C} \mathrm{NMR}\left(126 \mathrm{MHz}, \mathrm{CDCl}_{3}\right) \delta 201.9$, 154.3, 153.4, 133.6, 132.3, 131.6, 130.8, 124.2, 113.9, 62.0, 42.0, 29.3, 17.1, 13.6; HRMS (ESI) $\mathrm{m} / \mathrm{z}:[\mathrm{M}+\mathrm{Na}]^{+}$Calcd for $\mathrm{C}_{14} \mathrm{H}_{15} \mathrm{ClN}_{2} \mathrm{O}_{2} \mathrm{Na} 301.0714$; Found 301.0718.

\section{3-(1-Chloro-2-oxoheptyl)-1-methylquinoxalin-2(1H)-one (3c)}<smiles>CCCCCC(=O)C(C)(C)c1nc2ccccc2n(C)c1=O</smiles>

Purified by column chromatography using the mixture of petroleum ether and ethyl acetate $(\mathrm{PE} / \mathrm{EA}=3: 1)$ as eluant, white solid $\left(43 \mathrm{mg}, 70 \%\right.$ yield), m.p. $163-164{ }^{\circ} \mathrm{C} .{ }^{1} \mathrm{H}$ NMR $(500 \mathrm{MHz}$, $\left.\mathrm{CDCl}_{3}\right) \delta 7.90(\mathrm{dd}, J=8.0,1.2 \mathrm{~Hz}, 1 \mathrm{H}), 7.68-7.58(\mathrm{~m}, 1 \mathrm{H}), 7.43-7.37(\mathrm{~m}, 1 \mathrm{H}), 7.34(\mathrm{~d}, J=$ $8.4 \mathrm{~Hz}, 1 \mathrm{H}), 5.71(\mathrm{~s}, 1 \mathrm{H}), 3.71(\mathrm{~s}, 3 \mathrm{H}), 2.95(\mathrm{dt}, J=17.7,7.4 \mathrm{~Hz}, 1 \mathrm{H}), 2.81(\mathrm{dt}, J=17.8,7.3 \mathrm{~Hz}$, $1 \mathrm{H}), 1.75-1.65(\mathrm{~m}, 2 \mathrm{H}), 1.34(\mathrm{dd}, J=7.2,3.6 \mathrm{~Hz}, 4 \mathrm{H}), 0.90(\mathrm{t}, J=7.0 \mathrm{~Hz}, 3 \mathrm{H}) ;{ }^{13} \mathrm{C} \mathrm{NMR}(126$ $\left.\mathrm{MHz}, \mathrm{CDCl}_{3}\right) \delta 202.1,154.3,153.4,133.6,132.4,131.6,130.8,124.2,113.9,62.0,40.1,31.2$, 29.3, 23.3, 22.5, 13.9; HRMS (ESI) m/z: $[\mathrm{M}+\mathrm{Na}]^{+}$Calcd for $\mathrm{C}_{16} \mathrm{H}_{19} \mathrm{ClN}_{2} \mathrm{O}_{2} \mathrm{Na} 329.1027$; Found 329.1028 . 
<smiles>CC(C)CCC(=O)C(C)(C)c1nc2ccccc2n(C)c1=O</smiles>

Purified by column chromatography using the mixture of petroleum ether and ethyl acetate $(\mathrm{PE} / \mathrm{EA}=3: 1)$ as eluant, white solid (40 mg, 65\% yield), m.p. $131-132{ }^{\circ} \mathrm{C} .{ }^{1} \mathrm{H}$ NMR $(500 \mathrm{MHz}$, $\left.\mathrm{CDCl}_{3}\right) \delta 7.91(\mathrm{dd}, J=8.0,1.4 \mathrm{~Hz}, 1 \mathrm{H}), 7.66-7.60(\mathrm{~m}, 1 \mathrm{H}), 7.42-7.37(\mathrm{~m}, 1 \mathrm{H}), 7.35(\mathrm{~d}, J=$ $8.4 \mathrm{~Hz}, 1 \mathrm{H}), 5.72(\mathrm{~s}, 1 \mathrm{H}), 3.72(\mathrm{~s}, 3 \mathrm{H}), 3.02-2.92(\mathrm{~m}, 1 \mathrm{H}), 2.88-2.79(\mathrm{~m}, 1 \mathrm{H}), 1.64-1.57(\mathrm{~m}$, $3 \mathrm{H}), 0.92(\mathrm{dd}, J=6.2,2.9 \mathrm{~Hz}, 6 \mathrm{H}) ;{ }^{13} \mathrm{C} \mathrm{NMR}\left(126 \mathrm{MHz}, \mathrm{CDCl}_{3}\right) \delta 202.4,154.3,153.4,133.6$, 132.4, 131.6, 130.8, 124.2, 113.9, 61.9, 38.2, 32.4, 29.3, 27.6, 22.4; HRMS (ESI) m/z: [M+Na] $]^{+}$ Calcd for $\mathrm{C}_{16} \mathrm{H}_{19} \mathrm{ClN}_{2} \mathrm{O}_{2} \mathrm{Na} 329.1027$; Found 329.1027.

\section{3-(1-Chloro-2-oxoundecyl)-1-methylquinoxalin-2(1H)-one (3e)}<smiles>CCCCCCCCCC(=O)C(C)(C)c1nc2ccccc2n(C)c1=O</smiles>

Purified by column chromatography using the mixture of petroleum ether and ethyl acetate $(\mathrm{PE} / \mathrm{EA}=3: 1)$ as eluant, white solid $\left(49 \mathrm{mg}, 68 \%\right.$ yield), m.p. $168-169^{\circ} \mathrm{C} .{ }^{1} \mathrm{H} \mathrm{NMR}(500 \mathrm{MHz}$, $\left.\mathrm{CDCl}_{3}\right) \delta 7.90(\mathrm{~d}, J=8.0 \mathrm{~Hz}, 1 \mathrm{H}), 7.62(\mathrm{dd}, J=11.5,4.2 \mathrm{~Hz}, 1 \mathrm{H}), 7.39$ (t, $\left.J=7.6 \mathrm{~Hz}, 1 \mathrm{H}\right), 7.34$ $(\mathrm{d}, J=8.4 \mathrm{~Hz}, 1 \mathrm{H}), 5.71(\mathrm{~s}, 1 \mathrm{H}), 3.71(\mathrm{~s}, 3 \mathrm{H}), 2.95(\mathrm{dt}, J=17.6,7.4 \mathrm{~Hz}, 1 \mathrm{H}), 2.80(\mathrm{dt}, J=17.7$, $7.3 \mathrm{~Hz}, 1 \mathrm{H}), 1.72-1.66(\mathrm{~m}, 2 \mathrm{H}), 1.35-1.23(\mathrm{~m}, 12 \mathrm{H}), 0.88(\mathrm{t}, J=6.8 \mathrm{~Hz}, 3 \mathrm{H}) ;{ }^{13} \mathrm{C} \mathrm{NMR}(126$ $\left.\mathrm{MHz}, \mathrm{CDCl}_{3}\right) \delta 202.1,154.3,153.4,133.6,132.4,131.6,130.8,124.2,113.9,61.9,40.2,31.9$, 29.4, 29.4, 29.3, 29.3, 29.0, 23.6, 22.7, 14.1; HRMS (ESI) m/z: $[\mathrm{M}+\mathrm{Na}]^{+}$Calcd for $\mathrm{C}_{20} \mathrm{H}_{27} \mathrm{ClN}_{2} \mathrm{O}_{2} \mathrm{Na} 385.1653$; Found 385.1653.

\section{3-(1-Chloro-2-cyclopropyl-2-oxoethyl)-1-methylquinoxalin-2(1H)-one (3f)}<smiles>Cn1c(=O)c(C(C)(Cl)C(=O)C2CC2)nc2ccccc21</smiles>

Purified by column chromatography using the mixture of petroleum ether and ethyl acetate $(\mathrm{PE} / \mathrm{EA}=3: 1)$ as eluant, white solid $(34 \mathrm{mg}, 61 \%$ yield $)$, m.p. $117-118^{\circ} \mathrm{C} .{ }^{1} \mathrm{H} \mathrm{NMR}(500 \mathrm{MHz}$, $\left.\mathrm{CDCl}_{3}\right) \delta 7.91(\mathrm{~d}, J=7.9 \mathrm{~Hz}, 1 \mathrm{H}), 7.62(\mathrm{t}, J=7.6 \mathrm{~Hz}, 1 \mathrm{H}), 7.39(\mathrm{t}, J=7.6 \mathrm{~Hz}, 1 \mathrm{H}), 7.35(\mathrm{~d}, J=$ $8.3 \mathrm{~Hz}, 1 \mathrm{H}), 5.96(\mathrm{~s}, 1 \mathrm{H}), 3.72(\mathrm{~s}, 3 \mathrm{H}), 2.49-2.37(\mathrm{~m}, 1 \mathrm{H}), 1.23(\mathrm{~d}, J=21.2 \mathrm{~Hz}, 2 \mathrm{H}), 1.10-$ $0.95(\mathrm{~m}, 2 \mathrm{H}) ;{ }^{13} \mathrm{C} \mathrm{NMR}\left(126 \mathrm{MHz}, \mathrm{CDCl}_{3}\right) \delta 201.3,154.0,153.4,133.7,132.4,131.6,130.8$, 124.1, 113.9, 62.3, 29.4, 18.9, 12.8, 12.5; HRMS (ESI) m/z: $[\mathrm{M}+\mathrm{Na}]^{+}$Calcd for $\mathrm{C}_{14} \mathrm{H}_{13} \mathrm{ClN}_{2} \mathrm{O}_{2} \mathrm{Na} 299.0558$; Found 299.0559. 
<smiles>Cn1c(=O)c(C(C)(C)C(=O)C2CCCCC2)nc2ccccc21</smiles>

Purified by column chromatography using the mixture of petroleum ether and ethyl acetate $(\mathrm{PE} / \mathrm{EA}=3: 1)$ as eluant, white solid (41 mg, 64\% yield), m.p. $140-141^{\circ} \mathrm{C} .{ }^{1} \mathrm{H}$ NMR $(500 \mathrm{MHz}$, $\left.\mathrm{CDCl}_{3}\right) \delta 7.91(\mathrm{dd}, J=8.0,1.3 \mathrm{~Hz}, 1 \mathrm{H}), 7.64-7.59(\mathrm{~m}, 1 \mathrm{H}), 7.41-7.36(\mathrm{~m}, 1 \mathrm{H}), 7.34(\mathrm{~d}, J=$ $8.4 \mathrm{~Hz}, 1 \mathrm{H}), 5.91(\mathrm{~s}, 1 \mathrm{H}), 3.71(\mathrm{~s}, 3 \mathrm{H}), 2.98(\mathrm{tt}, J=11.4,3.4 \mathrm{~Hz}, 1 \mathrm{H}), 2.06(\mathrm{~d}, J=12.9 \mathrm{~Hz}, 1 \mathrm{H})$, $1.99(\mathrm{~d}, J=11.6 \mathrm{~Hz}, 1 \mathrm{H}), 1.81(\mathrm{dd}, J=12.8,3.3 \mathrm{~Hz}, 2 \mathrm{H}), 1.68(\mathrm{~d}, J=11.3 \mathrm{~Hz}, 1 \mathrm{H}), 1.49$ (dd, $J$ $=24.6,12.7 \mathrm{~Hz}, 2 \mathrm{H}), 1.37-1.28(\mathrm{~m}, 2 \mathrm{H}), 1.23(\mathrm{~d}, J=12.2 \mathrm{~Hz}, 1 \mathrm{H}) ;{ }^{13} \mathrm{C}$ NMR $(126 \mathrm{MHz}$, $\left.\mathrm{CDCl}_{3}\right) \delta 204.3,154.2,153.4,133.6,132.4,131.5,130.8,124.2,113.9,60.6,48.1,29.3,28.9$, 28.7, 25.7, 25.6, 25.5; HRMS (ESI) $\mathrm{m} / \mathrm{z}$ : $[\mathrm{M}+\mathrm{Na}]^{+}$Calcd for $\mathrm{C}_{17} \mathrm{H}_{19} \mathrm{ClN}_{2} \mathrm{O}_{2} \mathrm{Na}$ 341.1027; Found 341.1027 .

\section{3-(1-Chloro-2-oxo-2-phenylethyl)-1-methylquinoxalin-2(1H)-one (3h)}<smiles>Cn1c(=O)c(C(Cl)(Cl)C(=O)c2ccccc2)nc2ccccc21</smiles>

Purified by column chromatography using the mixture of petroleum ether and ethyl acetate $(\mathrm{PE} / \mathrm{EA}=3: 1)$ as eluant, white solid $\left(39 \mathrm{mg}, 62 \%\right.$ yield), m.p. $148-149^{\circ} \mathrm{C} .{ }^{1} \mathrm{H}$ NMR $(500 \mathrm{MHz}$, $\left.\mathrm{CDCl}_{3}\right) \delta 8.05(\mathrm{~d}, J=7.3 \mathrm{~Hz}, 2 \mathrm{H}), 7.93(\mathrm{dd}, J=8.0,1.3 \mathrm{~Hz}, 1 \mathrm{H}), 7.61(\mathrm{t}, J=7.9 \mathrm{~Hz}, 1 \mathrm{H}), 7.57$ $(\mathrm{t}, J=7.4 \mathrm{~Hz}, 1 \mathrm{H}), 7.46(\mathrm{t}, J=7.7 \mathrm{~Hz}, 2 \mathrm{H}), 7.37(\mathrm{t}, J=8.2 \mathrm{~Hz}, 1 \mathrm{H}), 7.33(\mathrm{~d}, J=8.4 \mathrm{~Hz}, 1 \mathrm{H})$, $6.87(\mathrm{~s}, 1 \mathrm{H}), 3.71(\mathrm{~s}, 3 \mathrm{H}) ;{ }^{13} \mathrm{C} \mathrm{NMR}\left(126 \mathrm{MHz}, \mathrm{CDCl}_{3}\right) \delta 190.2,153.6,153.5,134.6,133.8$, 133.5, 132.6, 131.6, 131.0, 129.0, 128.9, 124.2, 113.8, 58.2, 29.4; HRMS (ESI) m/z: [M+Na $]^{+}$ Calcd for $\mathrm{C}_{17} \mathrm{H}_{13} \mathrm{ClN}_{2} \mathrm{O}_{2} \mathrm{Na} 335.0558$; Found 335.0558.

\section{3-(2-(3-Bromophenyl)-1-chloro-2-oxoethyl)-1-methylquinoxalin-2(1H)-one (3i)}<smiles>Cn1c(=O)c(C(C)(C)C(=O)c2cccc(Br)c2)nc2ccccc21</smiles>

Purified by column chromatography using the mixture of petroleum ether and ethyl acetate $(\mathrm{PE} / \mathrm{EA}=3: 1)$ as eluant, white solid (38 mg, 49\% yield), m.p. $159-160{ }^{\circ} \mathrm{C} .{ }^{1} \mathrm{H}$ NMR $(500 \mathrm{MHz}$, $\left.\mathrm{CDCl}_{3}\right) \delta 8.21(\mathrm{~s}, 1 \mathrm{H}), 7.96(\mathrm{~d}, J=7.8 \mathrm{~Hz}, 2 \mathrm{H}), 7.70(\mathrm{~d}, J=7.9 \mathrm{~Hz}, 1 \mathrm{H}), 7.63(\mathrm{t}, J=7.8 \mathrm{~Hz}, 1 \mathrm{H})$, 7.40 (t, $J=7.7 \mathrm{~Hz}, 1 \mathrm{H}), 7.35(\mathrm{t}, J=7.6 \mathrm{~Hz}, 2 \mathrm{H}), 6.75(\mathrm{~s}, 1 \mathrm{H}), 3.72(\mathrm{~s}, 3 \mathrm{H}) ;{ }^{13} \mathrm{C}$ NMR $(126 \mathrm{MHz}$, $\left.\mathrm{CDCl}_{3}\right) \delta 189.1,153.6,153.0,136.6,136.4,133.5,132.6,132.0,131.7,131.0,130.4,127.4$, 124.3, 123.2, 113.9, 58.0, 29.4; HRMS (ESI) m/z: $[\mathrm{M}+\mathrm{Na}]^{+}$Calcd for $\mathrm{C}_{17} \mathrm{H}_{12} \mathrm{BrClN}_{2} \mathrm{O}_{2} \mathrm{Na}$ 412.9663; Found 412.9655. 
<smiles>Cn1c(=O)c(C(Cl)(Cl)C(=O)c2ccc(F)cc2)nc2ccccc21</smiles>

Purified by column chromatography using the mixture of petroleum ether and ethyl acetate $(\mathrm{PE} / \mathrm{EA}=3: 1)$ as eluant, white solid (39 mg, 59\% yield), m.p. 163-164 ${ }^{\circ} \mathrm{C} .{ }^{1} \mathrm{H}$ NMR $(500 \mathrm{MHz}$, $\left.\mathrm{CDCl}_{3}\right) \delta 8.09(\mathrm{dd}, J=8.8,5.3 \mathrm{~Hz}, 2 \mathrm{H}), 7.95(\mathrm{dd}, J=8.0,1.1 \mathrm{~Hz}, 1 \mathrm{H}), 7.66-7.59(\mathrm{~m}, 1 \mathrm{H}), 7.39$ $(\mathrm{dd}, J=11.3,4.1 \mathrm{~Hz}, 1 \mathrm{H}), 7.35(\mathrm{~d}, J=8.4 \mathrm{~Hz}, 1 \mathrm{H}), 7.14(\mathrm{t}, J=8.6 \mathrm{~Hz}, 2 \mathrm{H}), 6.80(\mathrm{~s}, 1 \mathrm{H}), 3.72$ $(\mathrm{s}, 3 \mathrm{H}) ;{ }^{13} \mathrm{C}$ NMR $\left(126 \mathrm{MHz}, \mathrm{CDCl}_{3}\right) \delta 188.8,166.1(\mathrm{~d}, J=257.0 \mathrm{~Hz}), 153.6,153.2,133.5$, $132.6,131.8(\mathrm{~d}, J=8.8 \mathrm{~Hz}), 131.6,131.0,130.9(\mathrm{~d}, J=3.8 \mathrm{~Hz}), 124.3,116.1(\mathrm{~d}, J=21.4 \mathrm{~Hz})$, 113.9, 58.0, 29.4; ${ }^{19} \mathrm{~F} \mathrm{NMR} \mathrm{(471} \mathrm{MHz,} \mathrm{CDCl}_{3}$ ) $\delta$-103.59; HRMS (ESI) m/z: [M+Na] ${ }^{+} \mathrm{Calcd}$ for $\mathrm{C}_{17} \mathrm{H}_{12} \mathrm{ClFN}_{2} \mathrm{O}_{2} \mathrm{Na} 353.0464$; Found 353.0457.

\section{3-(1-Chloro-2-(furan-2-yl)-2-oxoethyl)-1-methylquinoxalin-2(1H)-one (3k)}<smiles>Cn1c(=O)c(C(Cl)(Cl)C(=O)c2ccco2)nc2ccccc21</smiles>

Purified by column chromatography using the mixture of petroleum ether and ethyl acetate $(\mathrm{PE} / \mathrm{EA}=3: 1)$ as eluant, white solid $(28 \mathrm{mg}, 46 \%$ yield $)$, m.p. $152-153{ }^{\circ} \mathrm{C} .{ }^{1} \mathrm{H}$ NMR $(500 \mathrm{MHz}$, $\left.\mathrm{CDCl}_{3}\right) \delta 7.94(\mathrm{dd}, J=8.0,1.2 \mathrm{~Hz}, 1 \mathrm{H}), 7.62(\mathrm{t}, J=7.2 \mathrm{~Hz}, 1 \mathrm{H}), 7.59(\mathrm{~d}, J=1.1 \mathrm{~Hz}, 1 \mathrm{H}), 7.42$ $(\mathrm{d}, J=3.6 \mathrm{~Hz}, 1 \mathrm{H}), 7.39$ (dd, $J=11.3,4.0 \mathrm{~Hz}, 1 \mathrm{H}), 7.35$ (d, $J=8.4 \mathrm{~Hz}, 1 \mathrm{H}), 6.67(\mathrm{~s}, 1 \mathrm{H}), 6.57$ $(\mathrm{dd}, J=3.6,1.6 \mathrm{~Hz}, 1 \mathrm{H}), 3.73(\mathrm{~s}, 3 \mathrm{H}) ;{ }^{13} \mathrm{C} \mathrm{NMR}\left(126 \mathrm{MHz}, \mathrm{CDCl}_{3}\right) \delta 178.9,153.6,153.1,150.4$, 147.3, 133.5, 132.6, 131.6, 131.0, 124.2, 119.4, 113.8, 112.8, 58.1, 29.4; HRMS (ESI) m/z: $[\mathrm{M}+\mathrm{Na}]^{+}$Calcd for $\mathrm{C}_{15} \mathrm{H}_{11} \mathrm{ClN}_{2} \mathrm{O}_{3} \mathrm{Na} 325.0350$; Found 325.0351 .

\section{3-(1-Chloro-2-oxo-2-(thiophen-3-yl)ethyl)-1-methylquinoxalin-2(1H)-one (3I)}<smiles>Cn1c(=O)c(C(Cl)(Cl)C(=O)c2ccsc2)nc2ccccc21</smiles>

Purified by column chromatography using the mixture of petroleum ether and ethyl acetate $(\mathrm{PE} / \mathrm{EA}=3: 1)$ as eluant, white solid $\left(27 \mathrm{mg}, 42 \%\right.$ yield), m.p. $130-132{ }^{\circ} \mathrm{C} .{ }^{1} \mathrm{H}$ NMR $(500 \mathrm{MHz}$, $\left.\mathrm{CDCl}_{3}\right) \delta 8.27(\mathrm{~d}, J=1.6 \mathrm{~Hz}, 1 \mathrm{H}), 7.96(\mathrm{~d}, J=8.0 \mathrm{~Hz}, 1 \mathrm{H}), 7.63(\mathrm{dd}, J=9.8,5.9 \mathrm{~Hz}, 2 \mathrm{H}), 7.40$ $(\mathrm{t}, J=7.6 \mathrm{~Hz}, 1 \mathrm{H}), 7.34(\mathrm{dd}, J=10.1,5.7 \mathrm{~Hz}, 2 \mathrm{H}), 6.70(\mathrm{~s}, 1 \mathrm{H}), 3.73(\mathrm{~s}, 3 \mathrm{H}) ;{ }^{13} \mathrm{C} \mathrm{NMR}(126$ $\left.\mathrm{MHz}, \mathrm{CDCl}_{3}\right) \delta 184.0,153.6,153.3,139.1,133.8,133.5,132.7,131.6,131.1,127.6,126.7,124.3$, 113.8, 59.1, 29.4; HRMS (ESI) m/z: $[\mathrm{M}+\mathrm{Na}]^{+}$Calcd for $\mathrm{C}_{15} \mathrm{H}_{11} \mathrm{ClN}_{2} \mathrm{O}_{2} \mathrm{SNa}$ 341.0122; Found 341.0118 . 
1-Butyl-3-(1-chloro-2-oxopropyl)quinoxalin-2(1H)-one (3m)<smiles>CCCCn1c(=O)c(C(Cl)(Cl)C(C)=O)nc2ccccc21</smiles>

Purified by column chromatography using the mixture of petroleum ether and ethyl acetate $(\mathrm{PE} / \mathrm{EA}=3: 1)$ as eluant, white solid (43 mg, 73\% yield), m.p. $148-149^{\circ} \mathrm{C} .{ }^{1} \mathrm{H}$ NMR $(500 \mathrm{MHz}$, $\left.\mathrm{CDCl}_{3}\right) \delta 7.91(\mathrm{dd}, J=8.0,1.4 \mathrm{~Hz}, 1 \mathrm{H}), 7.62(\mathrm{ddd}, J=8.6,7.3,1.5 \mathrm{~Hz}, 1 \mathrm{H}), 7.41-7.33(\mathrm{~m}$, $2 \mathrm{H}), 5.68(\mathrm{~s}, 1 \mathrm{H}), 4.28-4.21(\mathrm{~m}, 2 \mathrm{H}), 2.56(\mathrm{~s}, 3 \mathrm{H}), 1.75(\mathrm{dd}, J=11.6,6.1 \mathrm{~Hz}, 2 \mathrm{H}), 1.48(\mathrm{dd}, J$ $=15.1,7.5 \mathrm{~Hz}, 2 \mathrm{H}), 1.00(\mathrm{t}, J=7.4 \mathrm{~Hz}, 3 \mathrm{H}) ;{ }^{13} \mathrm{C} \mathrm{NMR}\left(126 \mathrm{MHz}, \mathrm{CDCl}_{3}\right) \delta 200.2,154.2,153.1$, 132.9, 132.6, 131.6, 131.1, 124.0, 113.9, 62.3, 42.5, 29.3, 27.8, 20.3, 13.7; HRMS (ESI) m/z: $[\mathrm{M}+\mathrm{Na}]^{+}$Calcd for $\mathrm{C}_{15} \mathrm{H}_{17} \mathrm{ClN}_{2} \mathrm{O}_{2} \mathrm{Na} 315.0871$; Found 315.0870 .

\section{3-(1-Chloro-2-oxopropyl)-1-(cyclopropylmethyl)quinoxalin-2(1H)-one (3n)}<smiles>CC(=O)C(C)(Cl)c1nc2ccccc2n(CC2CC2)c1=O</smiles>

Purified by column chromatography using the mixture of petroleum ether and ethyl acetate $(\mathrm{PE} / \mathrm{EA}=3: 1)$ as eluant, white solid $(42 \mathrm{mg}, 72 \%$ yield $)$, m.p. $141-142{ }^{\circ} \mathrm{C} .{ }^{1} \mathrm{H}$ NMR $(500 \mathrm{MHz}$, $\left.\mathrm{CDCl}_{3}\right) \delta 7.91(\mathrm{dd}, J=8.0,1.4 \mathrm{~Hz}, 1 \mathrm{H}), 7.67-7.59(\mathrm{~m}, 1 \mathrm{H}), 7.47(\mathrm{~d}, J=7.9 \mathrm{~Hz}, 1 \mathrm{H}), 7.41-$ $7.35(\mathrm{~m}, 1 \mathrm{H}), 5.70(\mathrm{~s}, 1 \mathrm{H}), 4.20(\mathrm{~d}, J=7.0 \mathrm{~Hz}, 2 \mathrm{H}), 2.54(\mathrm{~s}, 3 \mathrm{H}), 1.31-1.25(\mathrm{~m}, 1 \mathrm{H}), 0.55(\mathrm{t}, J$ $=5.8 \mathrm{~Hz}, 4 \mathrm{H}) ;{ }^{13} \mathrm{C} \mathrm{NMR}\left(126 \mathrm{MHz}, \mathrm{CDCl}_{3}\right) \delta 200.1,154.4,153.4,133.1,132.5,131.5,131.0$, 124.0, 114.2, 62.3 , 46.5, 27.8, 9.6, 4.2; HRMS (ESI) m/z: $[\mathrm{M}+\mathrm{Na}]^{+}$Calcd for $\mathrm{C}_{15} \mathrm{H}_{15} \mathrm{ClN}_{2} \mathrm{O}_{2} \mathrm{Na}$ 313.0714; Found 313.0710.

\section{Methyl-2-(3-(1-chloro-2-oxopropyl)-2-oxoquinoxalin-1(2H)-yl)acetate (3o)}<smiles>COC(=O)Cn1c(=O)c(C(C)(Cl)C(C)=O)nc2ccccc21</smiles>

Purified by column chromatography using the mixture of petroleum ether and ethyl acetate $(\mathrm{PE} / \mathrm{EA}=3: 1)$ as eluant, white solid (43 mg, 70\% yield), m.p. $141-142{ }^{\circ} \mathrm{C} .{ }^{1} \mathrm{H}$ NMR $(500 \mathrm{MHz}$, $\left.\mathrm{CDCl}_{3}\right) \delta 7.94(\mathrm{dd}, J=8.0,1.3 \mathrm{~Hz}, 1 \mathrm{H}), 7.63-7.58(\mathrm{~m}, 1 \mathrm{H}), 7.44-7.39(\mathrm{~m}, 1 \mathrm{H}), 7.12(\mathrm{~d}, J=$ $8.0 \mathrm{~Hz}, 1 \mathrm{H}), 5.70(\mathrm{~s}, 1 \mathrm{H}), 5.05(\mathrm{~s}, 2 \mathrm{H}), 3.79(\mathrm{~s}, 3 \mathrm{H}), 2.54(\mathrm{~s}, 3 \mathrm{H}) ;{ }^{13} \mathrm{C} \mathrm{NMR}\left(126 \mathrm{MHz}, \mathrm{CDCl}_{3}\right)$ $\delta$ 199.9, 167.1, 154.0, 153.0, 132.8, 132.4, 131.9, 131.2, 124.6, 113.3, 62.0, 53.0, 43.6, 27.8; HRMS (ESI) m/z: [M+Na] ${ }^{+}$Calcd for $\mathrm{C}_{14} \mathrm{H}_{13} \mathrm{ClN}_{2} \mathrm{O}_{4} \mathrm{Na} 331.0456$; Found 331.0458. 
Tert-butyl-2-(3-(1-chloro-2-oxopropyl)-2-oxoquinoxalin-1(2H)-yl)acetate (3p)<smiles>CC(=O)C(Cl)c1nc2ccccc2n(CC(=O)OC(C)(C)C)c1=O</smiles>

Purified by column chromatography using the mixture of petroleum ether and ethyl acetate $(\mathrm{PE} / \mathrm{EA}=3: 1)$ as eluant, white solid (50 mg, 71\% yield), m.p. $131-132{ }^{\circ} \mathrm{C} .{ }^{1} \mathrm{H}$ NMR $(500 \mathrm{MHz}$, $\left.\mathrm{CDCl}_{3}\right) \delta 7.92(\mathrm{dd}, J=8.0,1.3 \mathrm{~Hz}, 1 \mathrm{H}), 7.62-7.57(\mathrm{~m}, 1 \mathrm{H}), 7.42-7.37(\mathrm{~m}, 1 \mathrm{H}), 7.12(\mathrm{~d}, J=$ $8.4 \mathrm{~Hz}, 1 \mathrm{H}), 5.72(\mathrm{~s}, 1 \mathrm{H}), 4.93(\mathrm{~d}, J=2.6 \mathrm{~Hz}, 2 \mathrm{H}), 2.52(\mathrm{~s}, 3 \mathrm{H}), 1.44(\mathrm{~s}, 9 \mathrm{H}) ;{ }^{13} \mathrm{C}$ NMR $(126$ $\left.\mathrm{MHz}, \mathrm{CDCl}_{3}\right) \delta 199.6,165.6,154.0,153.0,132.9,132.4,131.8,131.1,124.4,113.4,83.5,62.1$, 44.4, 27.9, 27.8; HRMS (ESI) m/z: [M+Na] ${ }^{+}$Calcd for $\mathrm{C}_{17} \mathrm{H}_{19} \mathrm{ClN}_{2} \mathrm{O}_{4} \mathrm{Na}$ 373.0926; Found 373.0937 .

\section{1-Benzyl-3-(1-chloro-2-oxopropyl)quinoxalin-2(1H)-one (3q)}<smiles>CC(=O)C(Cl)(Cl)c1nc2ccccc2n(Cc2ccccc2)c1=O</smiles>

Purified by column chromatography using the mixture of petroleum ether and ethyl acetate $(\mathrm{PE} / \mathrm{EA}=3: 1)$ as eluant, white solid $\left(45 \mathrm{mg}, 69 \%\right.$ yield), m.p. $175-176{ }^{\circ} \mathrm{C} .{ }^{1} \mathrm{H}$ NMR $(500 \mathrm{MHz}$, $\left.\mathrm{CDCl}_{3}\right) \delta 7.91(\mathrm{dd}, J=8.0,1.1 \mathrm{~Hz}, 1 \mathrm{H}), 7.51-7.46(\mathrm{~m}, 1 \mathrm{H}), 7.33(\mathrm{dd}, J=17.7,7.8 \mathrm{~Hz}, 3 \mathrm{H})$, $7.29-7.26(\mathrm{~m}, 2 \mathrm{H}), 7.22(\mathrm{~d}, J=7.4 \mathrm{~Hz}, 2 \mathrm{H}), 5.74(\mathrm{~s}, 1 \mathrm{H}), 5.49(\mathrm{~s}, 2 \mathrm{H}), 2.58(\mathrm{~s}, 3 \mathrm{H}) ;{ }^{13} \mathrm{C} \mathrm{NMR}$ $\left(126 \mathrm{MHz}, \mathrm{CDCl}_{3}\right) \delta 200.3,154.4,153.5,134.6,133.0,132.6,131.6,130.9,129.1,127.9,126.8$, 124.3, 114.7, 62.2, 46.2, 27.9; HRMS (ESI) m/z: $[\mathrm{M}+\mathrm{Na}]^{+}$Calcd for $\mathrm{C}_{18} \mathrm{H}_{15} \mathrm{ClN}_{2} \mathrm{O}_{2} \mathrm{Na}$ 349.0714; Found 349.0711.

\section{3-(1-Chloro-2-oxopropyl)-1-(2-fluorobenzyl)quinoxalin-2(1H)-one (3r)}<smiles>CC(=O)C(C)(Cl)c1nc2ccccc2n(Cc2ccccc2F)c1=O</smiles>

Purified by column chromatography using the mixture of petroleum ether and ethyl acetate $(\mathrm{PE} / \mathrm{EA}=3: 1)$ as eluant, white solid (38 mg, 55\% yield), m.p. $168-169^{\circ} \mathrm{C} .{ }^{1} \mathrm{H}$ NMR $(500 \mathrm{MHz}$, $\left.\mathrm{CDCl}_{3}\right) \delta 7.92(\mathrm{dd}, J=8.0,1.4 \mathrm{~Hz}, 1 \mathrm{H}), 7.54-7.50(\mathrm{~m}, 1 \mathrm{H}), 7.38-7.34(\mathrm{~m}, 1 \mathrm{H}), 7.28(\mathrm{dd}, J=$ 
12.3, 3.7 Hz, 2H), $7.13-7.09(\mathrm{~m}, 1 \mathrm{H}), 7.06-7.00(\mathrm{~m}, 2 \mathrm{H}), 5.73(\mathrm{~s}, 1 \mathrm{H}), 5.55(\mathrm{~s}, 2 \mathrm{H}), 2.59(\mathrm{~s}$, $3 \mathrm{H}) ;{ }^{13} \mathrm{C}$ NMR $\left(126 \mathrm{MHz}, \mathrm{CDCl}_{3}\right) \delta 200.3,160.3(\mathrm{~d}, J=247.0 \mathrm{~Hz}), 154.3,153.6,132.6(\mathrm{~d}, J=$ $11.3 \mathrm{~Hz}), 131.8,131.0,129.7(\mathrm{~d}, J=8.8 \mathrm{~Hz}), 128.5(\mathrm{~d}, J=3.8 \mathrm{~Hz}), 124.9$ (d, $J=3.8 \mathrm{~Hz}), 124.4$, 121.8, 121.7, $115.6(\mathrm{~d}, J=21.4 \mathrm{~Hz}), 114.3(\mathrm{~d}, J=2.5 \mathrm{~Hz}), 62.2,39.6(\mathrm{~d}, J=5.0 \mathrm{~Hz}), 27.9 ;{ }^{19} \mathrm{~F}$ NMR (471 MHz, $\mathrm{CDCl}_{3}$ ) $\delta$-118.33; HRMS (ESI) m/z: [M+Na] $]^{+}$Calcd for $\mathrm{C}_{18} \mathrm{H}_{14} \mathrm{ClFN}_{2} \mathrm{O}_{2} \mathrm{Na}$ 367.0620; Found 367.0649.

\section{3-(1-Chloro-2-oxopropyl)-1-(2-chlorobenzyl)quinoxalin-2(1H)-one (3s)}<smiles>CC(=O)C(C)(Cl)c1nc2ccccc2n(Cc2ccccc2Cl)c1=O</smiles>

Purified by column chromatography using the mixture of petroleum ether and ethyl acetate $(\mathrm{PE} / \mathrm{EA}=3: 1)$ as eluant, white solid (38 mg, 53\% yield), m.p. $138-139{ }^{\circ} \mathrm{C} .{ }^{1} \mathrm{H}$ NMR $(500 \mathrm{MHz}$, $\left.\mathrm{CDCl}_{3}\right) \delta 7.94(\mathrm{dd}, J=8.0,1.4 \mathrm{~Hz}, 1 \mathrm{H}), 7.52-7.47(\mathrm{~m}, 1 \mathrm{H}), 7.45(\mathrm{~d}, J=8.9 \mathrm{~Hz}, 1 \mathrm{H}), 7.37(\mathrm{t}, J$ $=7.2 \mathrm{~Hz}, 1 \mathrm{H}), 7.22(\mathrm{t}, J=7.1 \mathrm{~Hz}, 1 \mathrm{H}), 7.12(\mathrm{dd}, J=17.6,8.2 \mathrm{~Hz}, 2 \mathrm{H}), 6.73(\mathrm{~d}, J=7.7 \mathrm{~Hz}, 1 \mathrm{H})$, $5.73(\mathrm{~s}, 1 \mathrm{H}), 5.59(\mathrm{~s}, 2 \mathrm{H}), 2.60(\mathrm{~s}, 3 \mathrm{H}) ;{ }^{13} \mathrm{C} \mathrm{NMR}\left(126 \mathrm{MHz}, \mathrm{CDCl}_{3}\right) \delta 200.4,154.4,153.5$, 132.7, 132.6, 132.6, 131.9, 131.7, 131.0, 129.9, 129.1, 127.6, 126.8, 124.5, 114.6, 62.1, 43.8, 27.9; HRMS (ESI) m/z: [M+Na] ${ }^{+}$Calcd for $\mathrm{C}_{18} \mathrm{H}_{14} \mathrm{Cl}_{2} \mathrm{~N}_{2} \mathrm{O}_{2} \mathrm{Na} 383.0325$; Found 383.0320.

\section{3-(1-Chloro-2-oxopropyl)-1-(3-fluorobenzyl)quinoxalin-2(1H)-one (3t)}<smiles>CC(=O)C(Cl)(Cl)c1nc2ccccc2n(Cc2cccc(F)c2)c1=O</smiles>

Purified by column chromatography using the mixture of petroleum ether and ethyl acetate $(\mathrm{PE} / \mathrm{EA}=3: 1)$ as eluant, white solid $\left(40 \mathrm{mg}, 58 \%\right.$ yield), m.p. $165-166^{\circ} \mathrm{C} .{ }^{1} \mathrm{H}$ NMR $(500 \mathrm{MHz}$, $\left.\mathrm{CDCl}_{3}\right) \delta 7.91(\mathrm{dd}, J=8.0,1.2 \mathrm{~Hz}, 1 \mathrm{H}), 7.50(\mathrm{t}, J=7.9 \mathrm{~Hz}, 1 \mathrm{H}), 7.35(\mathrm{t}, J=7.6 \mathrm{~Hz}, 1 \mathrm{H}), 7.29$ (dd, $J=13.0,7.1 \mathrm{~Hz}, 1 \mathrm{H}), 7.23$ (d, $J=8.4 \mathrm{~Hz}, 1 \mathrm{H}), 7.00$ (d, $J=7.7 \mathrm{~Hz}, 1 \mathrm{H}), 6.97-6.90$ (m, $2 \mathrm{H}), 5.73(\mathrm{~s}, 1 \mathrm{H}), 5.47(\mathrm{~s}, 2 \mathrm{H}), 2.58(\mathrm{~s}, 3 \mathrm{H}) ;{ }^{13} \mathrm{C} \mathrm{NMR}\left(126 \mathrm{MHz}, \mathrm{CDCl}_{3}\right) \delta 200.2,163.1(\mathrm{~d}, J=$ $248.2 \mathrm{~Hz}), 154.3,153.4,137.1(\mathrm{~d}, J=7.6 \mathrm{~Hz}), 132.8,132.6,131.8,131.0,130.8$ (d, $J=7.6 \mathrm{~Hz})$, 124.4, 122.4 (d, $J=3.8 \mathrm{~Hz}), 115.0(\mathrm{~d}, J=21.4 \mathrm{~Hz}), 114.5,113.9$ (d, $J=22.7 \mathrm{~Hz}), 62.2,45.7$ (d, $J=1.3 \mathrm{~Hz}$ ), 27.9; ${ }^{19} \mathrm{~F} \mathrm{NMR}\left(471 \mathrm{MHz}, \mathrm{CDCl}_{3}\right) \delta-111.68$; HRMS (ESI) m/z: [M+Na] ${ }^{+} \mathrm{Calcd}$ for $\mathrm{C}_{18} \mathrm{H}_{14} \mathrm{ClFN}_{2} \mathrm{O}_{2} \mathrm{Na}$ 367.0620; Found 367.0620. 
<smiles>CC(=O)C(C)(Cl)c1nc2ccccc2n(Cc2ccc(F)cc2)c1=O</smiles>

Purified by column chromatography using the mixture of petroleum ether and ethyl acetate $(\mathrm{PE} / \mathrm{EA}=3: 1)$ as eluant, white solid $(43 \mathrm{mg}, 62 \%$ yield $)$, m.p. $127-128{ }^{\circ} \mathrm{C} .{ }^{1} \mathrm{H}$ NMR $(500 \mathrm{MHz}$, $\left.\mathrm{CDCl}_{3}\right) \delta 7.92(\mathrm{dd}, J=8.0,1.4 \mathrm{~Hz}, 1 \mathrm{H}), 7.51(\mathrm{ddd}, J=8.6,7.4,1.5 \mathrm{~Hz}, 1 \mathrm{H}), 7.38-7.34(\mathrm{~m}$, $1 \mathrm{H}), 7.27$ (d, $J=9.5 \mathrm{~Hz}, 1 \mathrm{H}), 7.22(\mathrm{dd}, J=8.6,5.2 \mathrm{~Hz}, 2 \mathrm{H}), 7.01(\mathrm{t}, J=8.6 \mathrm{~Hz}, 2 \mathrm{H}), 5.72$ (s, 1H), $5.46(\mathrm{~s}, 2 \mathrm{H}), 2.59(\mathrm{~s}, 3 \mathrm{H}) ;{ }^{13} \mathrm{C} \mathrm{NMR}\left(126 \mathrm{MHz}, \mathrm{CDCl}_{3}\right) \delta 200.3,162.3(\mathrm{~d}, J=247.0 \mathrm{~Hz})$, 154.4, 153.4, 132.8, 132.6, 131.7, 131.0, 130.4 (d, $J=2.5 \mathrm{~Hz}), 128.7$ (d, $J=7.6 \mathrm{~Hz}), 124.4$, $116.0(\mathrm{~d}, J=21.4 \mathrm{~Hz}), 114.5,62.2,45.5,27.9 ;{ }^{19} \mathrm{~F} \mathrm{NMR}\left(471 \mathrm{MHz}, \mathrm{CDCl}_{3}\right) \delta-113.91 ; \mathrm{HRMS}$ (ESI) $\mathrm{m} / \mathrm{z}$ : $[\mathrm{M}+\mathrm{Na}]^{+}$Calcd for $\mathrm{C}_{18} \mathrm{H}_{14} \mathrm{ClFN}_{2} \mathrm{O}_{2} \mathrm{Na} 367.0620$; Found 367.0617.

\section{3-(1-Chloro-2-oxopropyl)-1-(4-methoxybenzyl)quinoxalin-2(1H)-one (3v)}<smiles>COc1ccc(Cn2c(=O)c(C(Cl)(Cl)C(C)=O)nc3ccccc32)cc1</smiles>

Purified by column chromatography using the mixture of petroleum ether and ethyl acetate $(\mathrm{PE} / \mathrm{EA}=3: 1)$ as eluant, white solid $(50 \mathrm{mg}, 70 \%$ yield $)$, m.p. $142-143{ }^{\circ} \mathrm{C} .{ }^{1} \mathrm{H} \mathrm{NMR}(500 \mathrm{MHz}$, $\left.\mathrm{CDCl}_{3}\right) \delta 7.90(\mathrm{~d}, J=7.8 \mathrm{~Hz}, 1 \mathrm{H}), 7.50(\mathrm{t}, J=7.6 \mathrm{~Hz}, 1 \mathrm{H}), 7.34(\mathrm{t}, J=7.0 \mathrm{~Hz}, 2 \mathrm{H}), 7.18(\mathrm{~d}, J=$ $8.4 \mathrm{~Hz}, 2 \mathrm{H}), 6.84(\mathrm{~d}, J=8.5 \mathrm{~Hz}, 2 \mathrm{H}), 5.73(\mathrm{~s}, 1 \mathrm{H}), 5.43(\mathrm{~s}, 2 \mathrm{H}), 3.76(\mathrm{~s}, 3 \mathrm{H}), 2.58(\mathrm{~s}, 3 \mathrm{H}) ;{ }^{13} \mathrm{C}$ NMR $\left(126 \mathrm{MHz}, \mathrm{CDCl}_{3}\right) \delta 200.3,159.3,154.4,153.5,133.0,132.6,131.6,130.9,128.4,126.7$, 124.2, 114.7, 114.4, 62.2, 55.3, 45.7, 27.9; HRMS (ESI) $\mathrm{m} / \mathrm{z}:[\mathrm{M}+\mathrm{Na}]^{+}$Calcd for $\mathrm{C}_{19} \mathrm{H}_{17} \mathrm{ClN}_{2} \mathrm{O}_{3} \mathrm{Na}$ 379.0820; Found 379.0829.

\section{4-((3-(1-Chloro-2-oxopropyl)-2-oxoquinoxalin-1(2H)-yl)methyl)benzonitrile (3w)}<smiles>CC(=O)C(Cl)c1nc2ccccc2n(Cc2ccc(C#N)cc2)c1=O</smiles>

Purified by column chromatography using the mixture of petroleum ether and ethyl acetate $(\mathrm{PE} / \mathrm{EA}=3: 1)$ as eluant, white solid (37 mg, 53\% yield), m.p. $143-144{ }^{\circ} \mathrm{C} .{ }^{1} \mathrm{H}$ NMR $(500 \mathrm{MHz}$, 
$\left.\mathrm{CDCl}_{3}\right) \delta 7.94(\mathrm{dd}, J=8.0,1.1 \mathrm{~Hz}, 1 \mathrm{H}), 7.62(\mathrm{~d}, J=8.3 \mathrm{~Hz}, 2 \mathrm{H}), 7.54-7.50(\mathrm{~m}, 1 \mathrm{H}), 7.39(\mathrm{t}, J$ $=7.4 \mathrm{~Hz}, 1 \mathrm{H}), 7.33(\mathrm{~d}, J=8.2 \mathrm{~Hz}, 2 \mathrm{H}), 7.16(\mathrm{~d}, J=8.4 \mathrm{~Hz}, 1 \mathrm{H}), 5.70(\mathrm{~s}, 1 \mathrm{H}), 5.54(\mathrm{~s}, 2 \mathrm{H}), 2.59$ (s, 3H); ${ }^{13} \mathrm{C}$ NMR $\left(126 \mathrm{MHz}, \mathrm{CDCl}_{3}\right) \delta 200.4,154.4,153.3,140.0,132.9,132.6,132.6,131.9$, 131.3, 127.5, 124.7, 118.3, 114.1, 112.1, 62.1, 45.8, 27.9; HRMS (ESI) m/z: [M+Na] ${ }^{+}$Calcd for $\mathrm{C}_{19} \mathrm{H}_{14} \mathrm{ClN}_{3} \mathrm{O}_{2} \mathrm{Na}$ 374.0667; Found 374.0669.

\section{3-(1-Chloro-2-(4-methoxyphenyl)-2-oxoethyl)-1-phenylquinoxalin-2(1H)-one (3x)}<smiles>COc1ccc(C(=O)C(Cl)(Cl)c2nc3ccccc3n(-c3ccccc3)c2=O)cc1</smiles>

Purified by column chromatography using the mixture of petroleum ether and ethyl acetate $(\mathrm{PE} / \mathrm{EA}=3: 1)$ as eluant, white solid $\left(36 \mathrm{mg}, 45 \%\right.$ yield), m.p. $172-173{ }^{\circ} \mathrm{C} .{ }^{1} \mathrm{H}$ NMR $(500 \mathrm{MHz}$, $\left.\mathrm{CDCl}_{3}\right) \delta 8.06(\mathrm{~d}, J=8.9 \mathrm{~Hz}, 2 \mathrm{H}), 8.01(\mathrm{dd}, J=7.9,1.5 \mathrm{~Hz}, 1 \mathrm{H}), 7.63-7.58(\mathrm{~m}, 2 \mathrm{H}), 7.55(\mathrm{t}, J$ $=7.4 \mathrm{~Hz}, 1 \mathrm{H}), 7.42-7.37(\mathrm{~m}, 1 \mathrm{H}), 7.35(\mathrm{td}, J=7.7,1.4 \mathrm{~Hz}, 1 \mathrm{H}), 7.32-7.27(\mathrm{~m}, 2 \mathrm{H}), 6.93(\mathrm{~d}$, $J=8.9 \mathrm{~Hz}, 2 \mathrm{H}), 6.87(\mathrm{~s}, 1 \mathrm{H}), 6.73(\mathrm{dd}, J=8.2,1.2 \mathrm{~Hz}, 1 \mathrm{H}) ;{ }^{13} \mathrm{C} \mathrm{NMR}\left(126 \mathrm{MHz}, \mathrm{CDCl}_{3}\right) \delta$ $188.8,164.1,154.2,153.4,135.1,134.2,132.6,131.5,131.0,130.4,129.8,128.2,128.0,127.4$, 124.3, 115.6, 114.1, 57.0, 55.6; HRMS (ESI) m/z: $[\mathrm{M}+\mathrm{Na}]^{+}$Calcd for $\mathrm{C}_{23} \mathrm{H}_{17} \mathrm{ClN}_{2} \mathrm{O}_{3} \mathrm{Na}$ 427.0820; Found 427.0825.

\section{3-(1-Chloro-2-oxopropyl)-1,5-dimethylquinoxalin-2(1H)-one (3y)}<smiles>CC(=O)C(C)(Cl)c1nc2c(C)cccc2n(C)c1=O</smiles>

Purified by column chromatography using the mixture of petroleum ether and ethyl acetate $(\mathrm{PE} / \mathrm{EA}=3: 1)$ as eluant, white solid (38 mg, 72\% yield), m.p. $113-114{ }^{\circ} \mathrm{C} .{ }^{1} \mathrm{H}$ NMR $(500 \mathrm{MHz}$, $\left.\mathrm{CDCl}_{3}\right) \delta 7.50(\mathrm{t}, J=7.9 \mathrm{~Hz}, 1 \mathrm{H}), 7.24(\mathrm{~d}, J=7.3 \mathrm{~Hz}, 1 \mathrm{H}), 7.17(\mathrm{~d}, J=8.4 \mathrm{~Hz}, 1 \mathrm{H}), 5.69(\mathrm{~s}, 1 \mathrm{H})$, $3.71(\mathrm{~s}, 3 \mathrm{H}), 2.66(\mathrm{~s}, 3 \mathrm{H}), 2.57(\mathrm{~s}, 3 \mathrm{H}) ;{ }^{13} \mathrm{C} \mathrm{NMR}\left(126 \mathrm{MHz}, \mathrm{CDCl}_{3}\right) \delta 200.6,153.2,152.4$, $139.9,133.89,131.5,130.9,125.5,111.7,62.5,29.5,27.9,17.4 ;$ HRMS (ESI) m/z: $[\mathrm{M}+\mathrm{Na}]^{+}$ Calcd for $\mathrm{C}_{13} \mathrm{H}_{13} \mathrm{ClN}_{2} \mathrm{O}_{2} \mathrm{Na} 287.0558$; Found 287.0555.

\section{5-Chloro-3-(1-chloro-2-oxopropyl)-1-methylquinoxalin-2(1H)-one (3z)}<smiles>CC(=O)C(C)(C)c1nc2c(Cl)cccc2n(C)c1=O</smiles>

Purified by column chromatography using the mixture of petroleum ether and ethyl acetate $(\mathrm{PE} / \mathrm{EA}=3: 1)$ as eluant, white solid $(40 \mathrm{mg}, 70 \%$ yield $)$, m.p. $117-118^{\circ} \mathrm{C} .{ }^{1} \mathrm{H}$ NMR $(500 \mathrm{MHz}$, DMSO) $\delta 7.72-7.68(\mathrm{~m}, 1 \mathrm{H}), 7.64-7.59(\mathrm{~m}, 2 \mathrm{H}), 6.07(\mathrm{~s}, 1 \mathrm{H}), 3.67(\mathrm{~s}, 3 \mathrm{H}), 2.35(\mathrm{~s}, 3 \mathrm{H}) ;{ }^{13} \mathrm{C}$ 
NMR (126 MHz, DMSO) $\delta$ 198.7, 155.0, 153.0, 135.9, 133.9, 132.6, 128.4, 125.0, 115.1, 63.7, 30.3, 27.9; HRMS (ESI) m/z: [M+Na] ${ }^{+}$Calcd for $\mathrm{C}_{12} \mathrm{H}_{10} \mathrm{Cl}_{2} \mathrm{~N}_{2} \mathrm{O}_{2} \mathrm{Na}$ 307.0012; Found 307.0013. 3-(1-Chloro-2-oxopropyl)-6-fluoro-1-methylquinoxalin-2(1H)-one (3aa)<smiles>CC(=O)C(C)(C)c1nc2cc(F)ccc2n(C)c1=O</smiles>

Purified by column chromatography using the mixture of petroleum ether and ethyl acetate $(\mathrm{PE} / \mathrm{EA}=3: 1)$ as eluant, white solid (39 mg, 73\% yield), m.p. 136-137 ${ }^{\circ} \mathrm{C} .{ }^{1} \mathrm{H}$ NMR $(500 \mathrm{MHz}$, $\left.\mathrm{CDCl}_{3}\right) \delta 7.62(\mathrm{dd}, J=8.4,2.8 \mathrm{~Hz}, 1 \mathrm{H}), 7.42-7.36(\mathrm{~m}, 1 \mathrm{H}), 7.32(\mathrm{dd}, J=9.2,4.7 \mathrm{~Hz}, 1 \mathrm{H}), 5.65$ (s, 1H), $3.72(\mathrm{~s}, 3 \mathrm{H}), 2.57(\mathrm{~s}, 3 \mathrm{H}) ;{ }^{13} \mathrm{C}$ NMR $\left(126 \mathrm{MHz}, \mathrm{CDCl}_{3}\right) \delta 200.2,158.9(\mathrm{~d}, J=245.7 \mathrm{~Hz})$, 155.8, 153.0, 132.9 (d, $J=11.3 \mathrm{~Hz}), 130.4,119.5(\mathrm{~d}, J=24.0 \mathrm{~Hz}), 116.2(\mathrm{~d}, J=22.7 \mathrm{~Hz}), 115.1$ $(\mathrm{d}, J=7.6 \mathrm{~Hz}), 62.0,29.6,27.9 ;{ }^{19} \mathrm{~F}$ NMR $\left(471 \mathrm{MHz}, \mathrm{CDCl}_{3}\right) \delta-117.75 ; \mathrm{HRMS}(\mathrm{ESI}) \mathrm{m} / \mathrm{z}$ : $[\mathrm{M}+\mathrm{Na}]^{+}$Calcd for $\mathrm{C}_{12} \mathrm{H}_{10} \mathrm{ClFN}_{2} \mathrm{O}_{2} \mathrm{Na} 291.0307$; Found 291.0306 .

\section{6-Bromo-3-(1-chloro-2-oxopropyl)-1-methylquinoxalin-2(1H)-one (3ab)}<smiles>CC(=O)C(C)(Cl)c1nc2cc(Br)ccc2n(C)c1=O</smiles>

Purified by column chromatography using the mixture of petroleum ether and ethyl acetate $(\mathrm{PE} / \mathrm{EA}=3: 1)$ as eluant, white solid $\left(45 \mathrm{mg}, 68 \%\right.$ yield), m.p. $160-161{ }^{\circ} \mathrm{C} .{ }^{1} \mathrm{H}$ NMR $(500 \mathrm{MHz}$, $\left.\mathrm{CDCl}_{3}\right) \delta 8.07(\mathrm{~d}, J=2.2 \mathrm{~Hz}, 1 \mathrm{H}), 7.72(\mathrm{dd}, J=8.9,2.2 \mathrm{~Hz}, 1 \mathrm{H}), 7.23(\mathrm{~d}, J=8.9 \mathrm{~Hz}, 1 \mathrm{H}), 5.64$ $(\mathrm{s}, 1 \mathrm{H}), 3.70(\mathrm{~s}, 3 \mathrm{H}), 2.56(\mathrm{~s}, 3 \mathrm{H}) ;{ }^{13} \mathrm{C} \mathrm{NMR}\left(126 \mathrm{MHz}, \mathrm{CDCl}_{3}\right) \delta 200.1,155.6,153.0,134.4$, 133.1, 133.1, 132.8, 116.8, 115.3, 61.9, 29.5, 27.8; HRMS (ESI) $\mathrm{m} / \mathrm{z}:[\mathrm{M}+\mathrm{Na}]^{+} \mathrm{Calcd}$ for $\mathrm{C}_{12} \mathrm{H}_{10} \mathrm{BrClN}_{2} \mathrm{O}_{2} \mathrm{Na} 350.9506$; Found 350.9510.

\section{3-(1-Chloro-2-oxopropyl)-7-methoxy-1-methylquinoxalin-2(1H)-one (3ac)}<smiles>COc1ccc2nc(C(C)(Cl)C(C)=O)c(=O)n(C)c2c1</smiles>

Purified by column chromatography using the mixture of petroleum ether and ethyl acetate $(\mathrm{PE} / \mathrm{EA}=3: 1)$ as eluant, white solid $(41 \mathrm{mg}, 73 \%$ yield $)$, m.p. $112-113{ }^{\circ} \mathrm{C} .{ }^{1} \mathrm{H}$ NMR $(500 \mathrm{MHz}$, $\left.\mathrm{CDCl}_{3}\right) \delta 7.37(\mathrm{~d}, J=2.5 \mathrm{~Hz}, 1 \mathrm{H}), 7.27-7.23(\mathrm{~m}, 2 \mathrm{H}), 5.71(\mathrm{~s}, 1 \mathrm{H}), 3.89(\mathrm{~s}, 3 \mathrm{H}), 3.71(\mathrm{~s}, 3 \mathrm{H})$, $2.55(\mathrm{~s}, 3 \mathrm{H}) ;{ }^{13} \mathrm{C}$ NMR $\left(126 \mathrm{MHz}, \mathrm{CDCl}_{3}\right) \delta 200.1,156.4,154.6,153.0,133.2,127.9,121.3$, 114.8, 111.8, 62.2, 55.9, 29.5, 27.9; HRMS (ESI) m/z: $[\mathrm{M}+\mathrm{Na}]^{+}$Calcd for $\mathrm{C}_{13} \mathrm{H}_{13} \mathrm{ClN}_{2} \mathrm{O}_{3} \mathrm{Na}$ 303.0507; Found 303.0521. 


\section{3-(1-Chloro-2-oxopropyl)-1,6,7-trimethylquinoxalin-2(1H)-one (3ad)}<smiles>CC(=O)c1nc2cc(C)c(C)cc2n(C)c1=O</smiles>

Purified by column chromatography using the mixture of petroleum ether and ethyl acetate $(\mathrm{PE} / \mathrm{EA}=3: 1)$ as eluant, white solid $(39 \mathrm{mg}, 70 \%$ yield $)$, m.p. $156-157{ }^{\circ} \mathrm{C} .{ }^{1} \mathrm{H}$ NMR $(500 \mathrm{MHz}$, $\left.\mathrm{CDCl}_{3}\right) \delta 7.65(\mathrm{~s}, 1 \mathrm{H}), 7.11(\mathrm{~s}, 1 \mathrm{H}), 5.68(\mathrm{~s}, 1 \mathrm{H}), 3.69(\mathrm{~s}, 3 \mathrm{H}), 2.54(\mathrm{~s}, 3 \mathrm{H}), 2.44(\mathrm{~s}, 3 \mathrm{H}), 2.36(\mathrm{~s}$, $3 \mathrm{H}) ;{ }^{13} \mathrm{C} \mathrm{NMR}\left(126 \mathrm{MHz}, \mathrm{CDCl}_{3}\right) \delta 200.3,153.5,152.9,142.0,133.3,131.8,130.9,130.8,114.4$, 62.5, 29.2, 27.8, 20.8, 19.2; HRMS (ESI) m/z: $[\mathrm{M}+\mathrm{Na}]^{+}$Calcd for $\mathrm{C}_{14} \mathrm{H}_{15} \mathrm{ClN}_{2} \mathrm{O}_{2} \mathrm{Na}$ 301.0714; Found 301.0715 .

\section{3-(1-Chloro-2-(4-isobutylphenyl)-2-oxoethyl)-1-methylquinoxalin-2(1H)-one (3ae)}<smiles>CC(C)Cc1ccc(C(=O)C(C)(Cl)c2nc3ccccc3n(C)c2=O)cc1</smiles>

Purified by column chromatography using the mixture of petroleum ether and ethyl acetate $(\mathrm{PE} / \mathrm{EA}=3: 1)$ as eluant, white solid (34 mg, 46\% yield), m.p. $150-151{ }^{\circ} \mathrm{C} .{ }^{1} \mathrm{H}$ NMR $(500 \mathrm{MHz}$, $\left.\mathrm{CDCl}_{3}\right) \delta 7.97(\mathrm{~d}, J=8.2 \mathrm{~Hz}, 2 \mathrm{H}), 7.94(\mathrm{dd}, J=8.0,1.0 \mathrm{~Hz}, 1 \mathrm{H}), 7.63-7.56(\mathrm{~m}, 1 \mathrm{H}), 7.37(\mathrm{t}, J$ $=7.3 \mathrm{~Hz}, 1 \mathrm{H}), 7.35-7.32(\mathrm{~m}, 1 \mathrm{H}), 7.24(\mathrm{~d}, J=8.2 \mathrm{~Hz}, 2 \mathrm{H}), 6.86(\mathrm{~s}, 1 \mathrm{H}), 3.71(\mathrm{~s}, 3 \mathrm{H}), 2.51(\mathrm{~d}$, $J=7.2 \mathrm{~Hz}, 2 \mathrm{H}), 1.93-1.84(\mathrm{~m}, 1 \mathrm{H}), 0.89(\mathrm{~d}, J=6.6 \mathrm{~Hz}, 6 \mathrm{H}) ;{ }^{13} \mathrm{C} \mathrm{NMR}\left(126 \mathrm{MHz}, \mathrm{CDCl}_{3}\right) \delta$ $189.8,153.6,153.6,148.5,133.5,132.6,132.2$, 131.5, 131.0, 129.6, 129.0, 124.2, 113.8, 58.0, 45.5, 30.1 , 29.4, 22.4; HRMS (ESI) m/z: [M+Na] $]^{+}$Calcd for $\mathrm{C}_{21} \mathrm{H}_{21} \mathrm{ClN}_{2} \mathrm{O}_{2} \mathrm{Na}$ 391.1184; Found 391.1189 .

\section{1-Methyl-3-(1-morpholino-2-oxopropyl)quinoxalin-2(1H)-one (4a)}<smiles>CC(=O)C(c1nc2ccccc2n(C)c1=O)N1CCOCC1</smiles>

Purified by column chromatography using the mixture of petroleum ether and ethyl acetate $(\mathrm{PE} / \mathrm{EA}=4: 1)$ as eluant, yellow solid (56 mg, 93\% yield), m.p. $157-158{ }^{\circ} \mathrm{C} .{ }^{1} \mathrm{H} \mathrm{NMR}(500 \mathrm{MHz}$, $\left.\mathrm{CDCl}_{3}\right) \delta 7.92(\mathrm{dd}, J=8.0,1.4 \mathrm{~Hz}, 1 \mathrm{H}), 7.60(\mathrm{ddd}, J=8.6,7.4,1.5 \mathrm{~Hz}, 1 \mathrm{H}), 7.40-7.36(\mathrm{~m}$, $1 \mathrm{H}), 7.34(\mathrm{~d}, J=8.4 \mathrm{~Hz}, 1 \mathrm{H}), 5.07(\mathrm{~s}, 1 \mathrm{H}), 3.79-3.74(\mathrm{~m}, 4 \mathrm{H}), 3.72(\mathrm{~s}, 3 \mathrm{H}), 2.87$ (dd, $J=8.4$, $3.7 \mathrm{~Hz}, 4 \mathrm{H}), 2.31(\mathrm{~s}, 3 \mathrm{H}) ;{ }^{13} \mathrm{C} \mathrm{NMR}\left(126 \mathrm{MHz}, \mathrm{CDCl}_{3}\right) \delta 204.6,155.4,154.7,133.1,132.4$, 
131.0, 130.7, 124.0, 113.8, 74.0, 67.5, 50.9, 29.3, 28.9; HRMS (ESI) m/z: $[\mathrm{M}+\mathrm{Na}]^{+}$Calcd for $\mathrm{C}_{16} \mathrm{H}_{19} \mathrm{~N}_{3} \mathrm{O}_{3} \mathrm{Na} 324.1319$; Found 324.1320.

\section{Diethyl-(1-(4-methyl-3-oxo-3,4-dihydroquinoxalin-2-yl)-2-oxopropyl)phosphonate (4b)}<smiles>CCOP(=O)(OCC)C(C(C)=O)c1nc2ccccc2n(C)c1=O</smiles>

Purified by column chromatography using the mixture of petroleum ether and ethyl acetate $(\mathrm{PE} / \mathrm{EA}=4: 1)$ as eluant, yellow solid (60 mg, 85\% yield), m.p. $166-167{ }^{\circ} \mathrm{C} .{ }^{1} \mathrm{H}$ NMR $(500 \mathrm{MHz}$, $\left.\mathrm{CDCl}_{3}\right) \delta 7.80(\mathrm{t}, J=8.8 \mathrm{~Hz}, 1 \mathrm{H}), 7.51(\mathrm{t}, J=7.8 \mathrm{~Hz}, 1 \mathrm{H}), 7.33(\mathrm{t}, J=7.6 \mathrm{~Hz}, 1 \mathrm{H}), 7.28(\mathrm{~d}, J=$ $8.4 \mathrm{~Hz}, 1 \mathrm{H}), 7.05(\mathrm{~s}, 1 \mathrm{H}), 4.27(\mathrm{dd}, J=14.5,7.2 \mathrm{~Hz}, 4 \mathrm{H}), 3.70(\mathrm{~s}, 3 \mathrm{H}), 2.64(\mathrm{~s}, 3 \mathrm{H}), 1.41(\mathrm{t}, J=$ $7.1 \mathrm{~Hz}, 6 \mathrm{H}) ;{ }^{13} \mathrm{C}$ NMR $\left(126 \mathrm{MHz}, \mathrm{CDCl}_{3}\right) \delta 200.7,164.5,164.2,161.9,157.3,157.2,156.3$, 154.9, 154.8, 152.7, 132.9, 129.8, 129.7, 123.7, 113.6, 108.9, 64.7, 64.6, 29.3, 19.2, 16.2, 16.1; ${ }^{31} \mathrm{P}$ NMR $\left(202 \mathrm{MHz}, \mathrm{CDCl}_{3}\right) \delta$-7.5; HRMS (ESI) m/z: $[\mathrm{M}+\mathrm{Na}]^{+}$Calcd for $\mathrm{C}_{16} \mathrm{H}_{21} \mathrm{~N}_{2} \mathrm{O}_{5} \mathrm{PNa}$ 375.1080; Found 375.1081.

\section{3-(Chloromethyl)-1-methylquinoxalin-2(1H)-one (4c)}<smiles>Cn1c(=O)c(CCl)nc2ccccc21</smiles>

Purified by column chromatography using the mixture of petroleum ether and ethyl acetate $(\mathrm{PE} / \mathrm{EA}=4: 1)$ as eluant, yellow solid $(38 \mathrm{mg}, 91 \%$ yield $)$, m.p. $99-100{ }^{\circ} \mathrm{C} .{ }^{1} \mathrm{H} \mathrm{NMR}(500 \mathrm{MHz}$, $\left.\mathrm{CDCl}_{3}\right) \delta 7.91(\mathrm{dd}, J=8.0,1.1 \mathrm{~Hz}, 1 \mathrm{H}), 7.65-7.58(\mathrm{~m}, 1 \mathrm{H}), 7.42-7.37(\mathrm{~m}, 1 \mathrm{H}), 7.34(\mathrm{~d}, J=$ $8.4 \mathrm{~Hz}, 1 \mathrm{H}), 4.79(\mathrm{~s}, 2 \mathrm{H}), 3.74(\mathrm{~s}, 3 \mathrm{H}) ;{ }^{13} \mathrm{C} \mathrm{NMR}\left(126 \mathrm{MHz}, \mathrm{CDCl}_{3}\right) \delta 154.5,153.9,133.8$, 132.4, 131.3, 130.6, 124.1, 113.8, 42.9, 29.2; HRMS (ESI) $\mathrm{m} / \mathrm{z}:[\mathrm{M}+\mathrm{Na}]^{+}$Calcd for $\mathrm{C}_{10} \mathrm{H}_{9} \mathrm{ClN}_{2} \mathrm{ONa} 231.0296$; Found 231.0304.

\section{1-Methyl-3-(tosylmethyl)quinoxalin-2(1H)-one (4d)}<smiles>Cc1ccc(S(=O)(=O)Cc2nc3ccccc3n(C)c2=O)cc1</smiles>

Purified by column chromatography using the mixture of petroleum ether and ethyl acetate (PE/EA $=4: 1)$ as eluant, yellow solid (51 mg, 78\% yield), m.p. $127-128{ }^{\circ} \mathrm{C} .{ }^{1} \mathrm{H} \mathrm{NMR}(500 \mathrm{MHz}$, $\left.\mathrm{CDCl}_{3}\right) \delta 7.79(\mathrm{t}, J=8.7 \mathrm{~Hz}, 3 \mathrm{H}), 7.61(\mathrm{t}, J=7.8 \mathrm{~Hz}, 1 \mathrm{H}), 7.36(\mathrm{t}, J=7.6 \mathrm{~Hz}, 1 \mathrm{H}), 7.34-7.29$ $(\mathrm{m}, 3 \mathrm{H}), 4.83(\mathrm{~s}, 2 \mathrm{H}), 3.66(\mathrm{~s}, 3 \mathrm{H}), 2.45(\mathrm{~s}, 3 \mathrm{H}) ;{ }^{13} \mathrm{C} \mathrm{NMR}\left(126 \mathrm{MHz}, \mathrm{CDCl}_{3}\right) \delta 154.4,148.9$, $144.9,136.9,133.6,132.6,131.5,130.7,129.7,128.6,124.0,113.8,59.4,29.5,21.7$; HRMS (ESI) $\mathrm{m} / \mathrm{z}$ : $[\mathrm{M}+\mathrm{Na}]^{+}$Calcd for $\mathrm{C}_{17} \mathrm{H}_{16} \mathrm{~N}_{2} \mathrm{O}_{3} \mathrm{SNa} 351.0774$; Found 351.0774. 


\section{1-Methyl-3-(2-oxopropylidene)-3,4-dihydroquinoxalin-2(1H)-one (5) ${ }^{1}$}<smiles>CC(=O)/C=C1\Nc2ccccc2N(C)C1=O</smiles>

Purified by column chromatography using the mixture of petroleum ether and ethyl acetate $(\mathrm{PE} / \mathrm{EA}=3: 1)$ as eluant, yellow solid (34 mg, 79\% yield). ${ }^{1} \mathrm{H} \mathrm{NMR}\left(500 \mathrm{MHz}, \mathrm{CDCl}_{3}\right) \delta 13.32$ (s, 1H), $7.14(\mathrm{~d}, J=14.5 \mathrm{~Hz}, 4 \mathrm{H}), 6.27(\mathrm{~s}, 1 \mathrm{H}), 3.63$ (s, 3H), 2.27 (s, 3H).

\section{1-Methyl-3-(2-oxopropyl)-3,4-dihydroquinoxalin-2(1H)-one $(6)^{2}$}<smiles>CC(=O)CC1Nc2ccccc2N(C)C1=O</smiles>

Purified by column chromatography using the mixture of petroleum ether and ethyl acetate $(\mathrm{PE} / \mathrm{EA}=3: 1)$ as eluant, yellow liquid $\left(18 \mathrm{mg}, 41 \%\right.$ yield). ${ }^{1} \mathrm{H} \mathrm{NMR}\left(500 \mathrm{MHz}, \mathrm{CDCl}_{3}\right) \delta 6.91$ $(\mathrm{dd}, J=13.3,7.1 \mathrm{~Hz}, 2 \mathrm{H}), 6.87-6.79(\mathrm{~m}, 1 \mathrm{H}), 6.70(\mathrm{~d}, J=7.5 \mathrm{~Hz}, 1 \mathrm{H}), 4.68(\mathrm{~s}, 1 \mathrm{H}), 4.28(\mathrm{~d}, J$ $=9.2 \mathrm{~Hz}, 1 \mathrm{H}), 3.32(\mathrm{~d}, J=24.8 \mathrm{~Hz}, 4 \mathrm{H}), 2.79(\mathrm{dd}, J=18.5,10.2 \mathrm{~Hz}, 1 \mathrm{H}), 2.21(\mathrm{~s}, 3 \mathrm{H})$.

1-Methyl-3-(2-oxopentylidene)-3,4-dihydroquinoxalin-2(1H)-one $(7)^{3}$<smiles>CCCC(=O)/C=C1\Nc2ccccc2N(C)C1=O</smiles>

Purified by column chromatography using the mixture of petroleum ether and ethyl acetate $\left(\mathrm{PE} / \mathrm{EA}=3: 1\right.$ ) as eluant, yellow solid (40 mg, 82\% yield). ${ }^{1} \mathrm{H} \mathrm{NMR}\left(500 \mathrm{MHz}, \mathrm{CDCl}_{3}\right) \delta 13.35$ $(\mathrm{s}, 1 \mathrm{H}), 7.13(\mathrm{~d}, J=2.7 \mathrm{~Hz}, 3 \mathrm{H}), 7.10-7.06(\mathrm{~m}, 1 \mathrm{H}), 6.24(\mathrm{~s}, 1 \mathrm{H}), 3.60(\mathrm{~s}, 3 \mathrm{H}), 2.47(\mathrm{t}, J=7.5$ $\mathrm{Hz}, 2 \mathrm{H}), 1.69$ (dd, $J=14.8,7.4 \mathrm{~Hz}, 2 \mathrm{H}), 0.96$ (t, $J=7.4 \mathrm{~Hz}, 3 \mathrm{H})$.

\section{1-Methyl-3-(2-oxopentan-3-yl)quinoxalin-2(1H)-one (8) ${ }^{1}$}<smiles>CCC(C(C)=O)c1nc2ccccc2n(C)c1=O</smiles>

Purified by column chromatography using the mixture of petroleum ether and ethyl acetate $(\mathrm{PE} / \mathrm{EA}=3: 1)$ as eluant, white solid (4 mg, 8\% yield). ${ }^{1} \mathrm{H} \mathrm{NMR}\left(500 \mathrm{MHz}, \mathrm{CDCl}_{3}\right) \delta 7.89(\mathrm{~d}, J$ $=7.9 \mathrm{~Hz}, 1 \mathrm{H}), 7.56(\mathrm{t}, J=7.7 \mathrm{~Hz}, 1 \mathrm{H}), 7.36(\mathrm{t}, J=7.6 \mathrm{~Hz}, 1 \mathrm{H}), 7.32(\mathrm{~d}, J=8.4 \mathrm{~Hz}, 1 \mathrm{H}), 4.31(\mathrm{t}$, 
$J=6.9 \mathrm{~Hz}, 1 \mathrm{H}), 3.71(\mathrm{~s}, 3 \mathrm{H}), 2.33(\mathrm{~s}, 3 \mathrm{H}), 2.10(\mathrm{dq}, J=20.3,6.8 \mathrm{~Hz}, 2 \mathrm{H}), 1.02(\mathrm{t}, J=7.4 \mathrm{~Hz}$, $3 \mathrm{H})$. 


\section{References}

1. Xu, J.; Yang, H.; He, L.; Huang, L.; Shen, J.; Li, W.; Zhang, P. Org. Lett. 2021, 23, 195.

2. Rostoll-Berenguer, J.; Blay, G.; Muñoz, M. C.; Pedro, J. R.; Vila, C. Org. Lett. 2019, 21, 6011.

3. Xu, J.; Huang, L.; He, L.; Ni, Z.; Shen, J.; Li, X.; Chen, K.; Li, W.; Zhang, P. Green Chem. 2021, 23, 2123. 


\section{Copies of NMR Spectra}

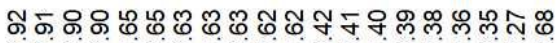

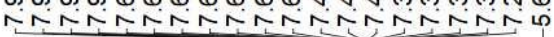<smiles>CC(=O)C(Cl)(Cl)c1nc2ccccc2n(C)c1=O</smiles>

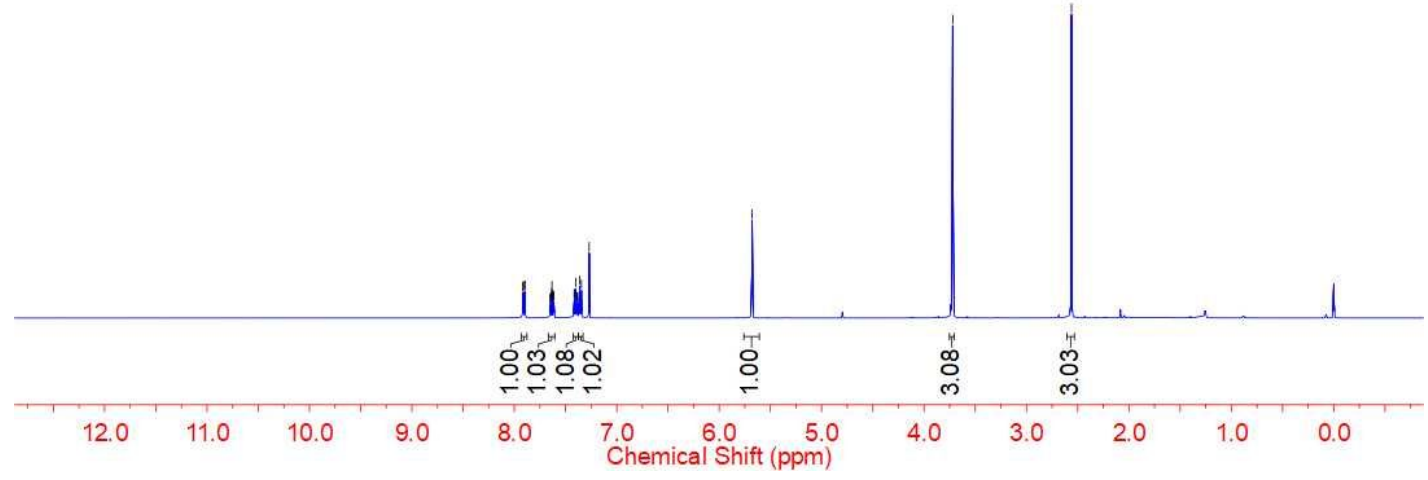

Figure S1. ${ }^{1} \mathrm{H} \mathrm{NMR}\left(500 \mathrm{MHz}, \mathrm{CDCl}_{3}\right)$ spectrum of $\mathbf{3 a}$.

กั่

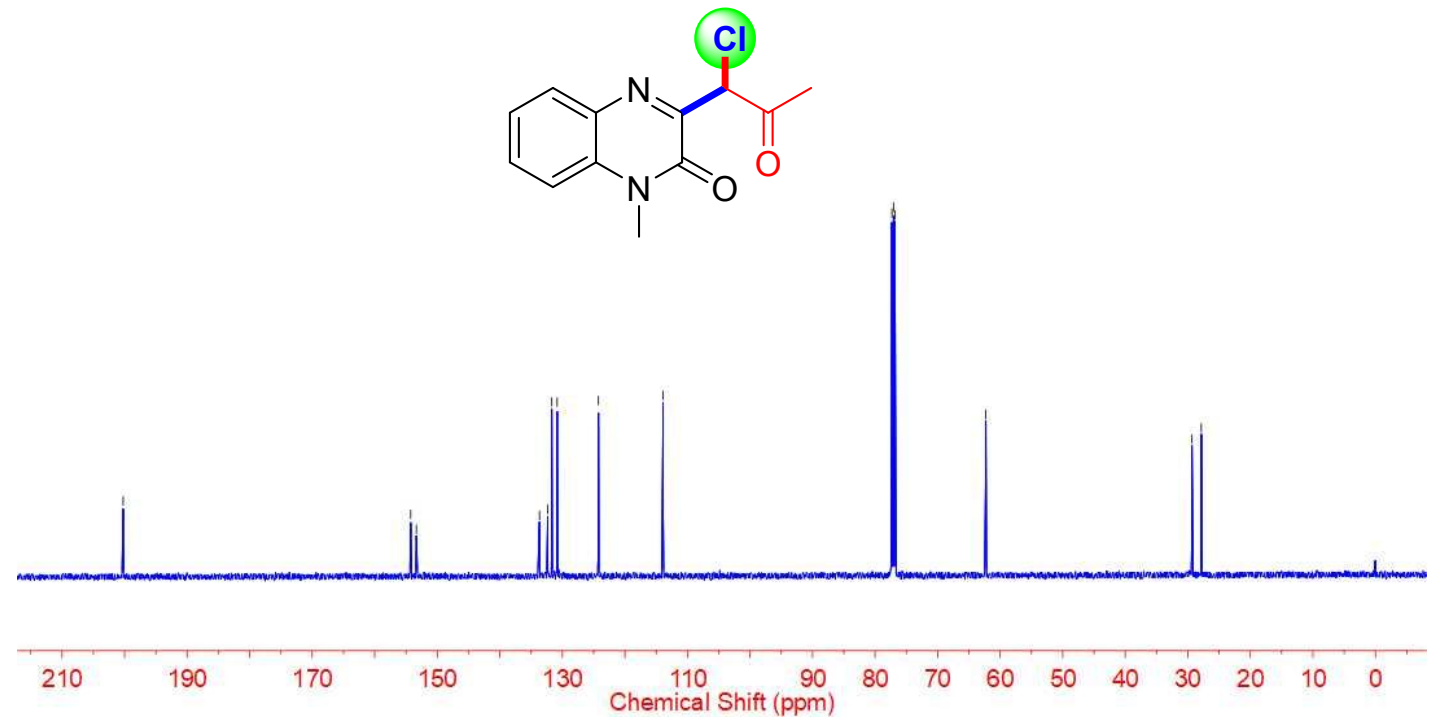

Figure S2. ${ }^{13} \mathrm{C}$ NMR $\left(126 \mathrm{MHz}, \mathrm{CDCl}_{3}\right)$ spectrum of 3a. 


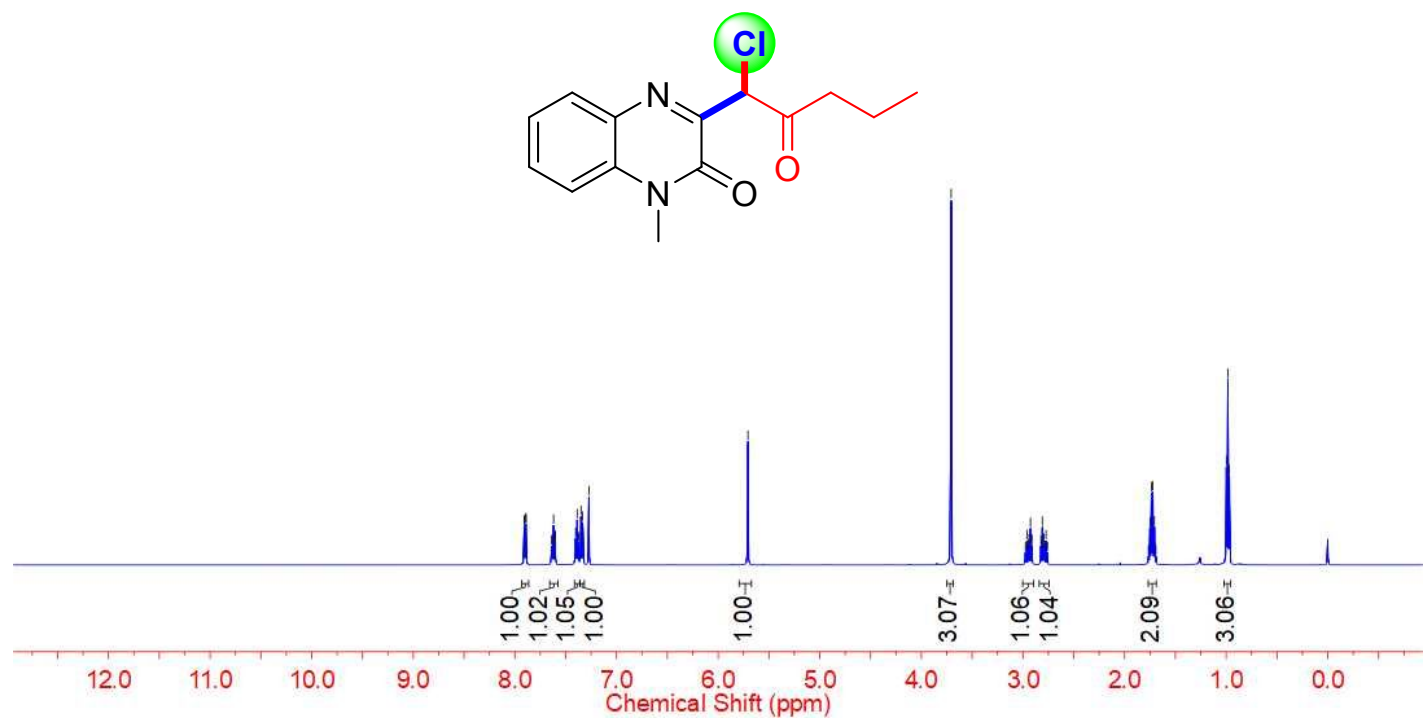

Figure S3. ${ }^{1} \mathrm{H}$ NMR $\left(500 \mathrm{MHz}, \mathrm{CDCl}_{3}\right)$ spectrum of $\mathbf{3 b}$.

त)<smiles>CCCC(=O)C(C)(Cl)c1nc2ccccc2n(C)c1=O</smiles>

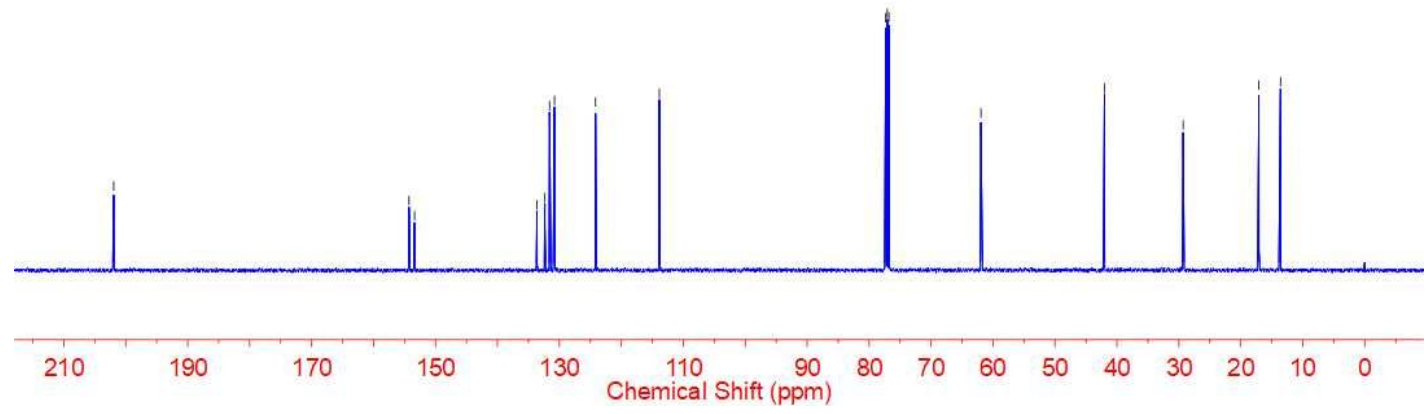

Figure S4. ${ }^{13} \mathrm{C}$ NMR (126 $\left.\mathrm{MHz}, \mathrm{CDCl}_{3}\right)$ spectrum of $\mathbf{3 b}$. 
<smiles>CCCCCC(=O)C(C)(C)c1nc2ccccc2n(C)c1=O</smiles>

Figure S5. ${ }^{1} \mathrm{H}$ NMR $\left(500 \mathrm{MHz}, \mathrm{CDCl}_{3}\right)$ spectrum of 3c.

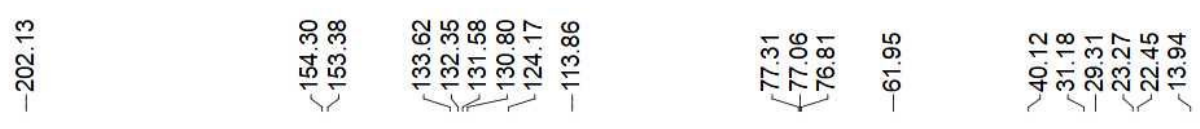<smiles>CCCCCC(=O)C(C)(C)c1nc2ccccc2n(C)c1=O</smiles>

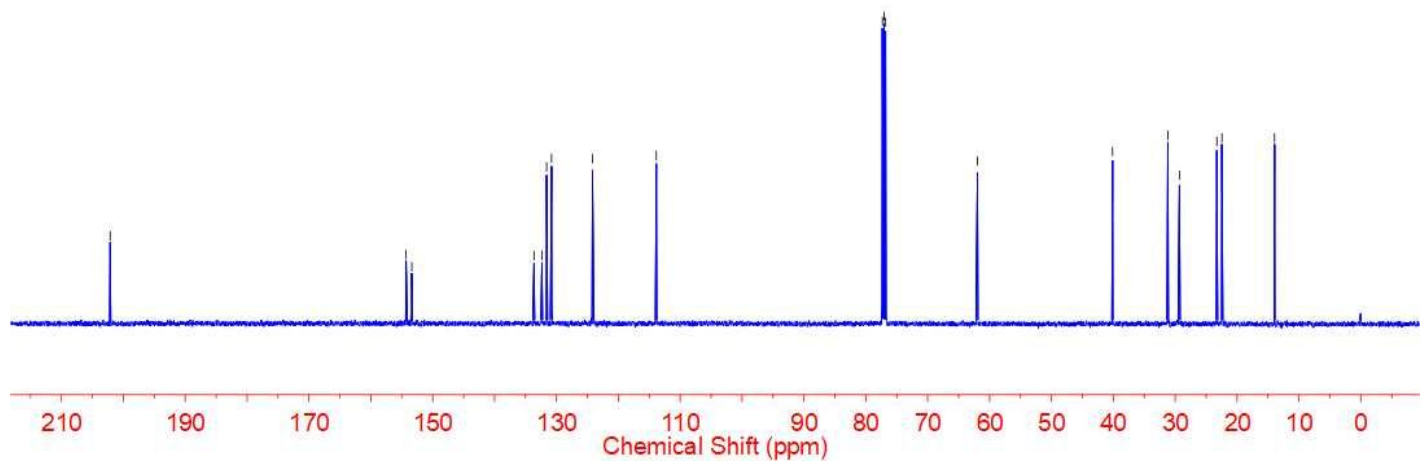

Figure S6. ${ }^{13} \mathrm{C}$ NMR $\left(126 \mathrm{MHz}, \mathrm{CDCl}_{3}\right)$ spectrum of $3 \mathbf{c}$. 
<smiles>CC(C)CCC(=O)C(Cl)(Cl)c1nc2ccccc2n(C)c1=O</smiles>

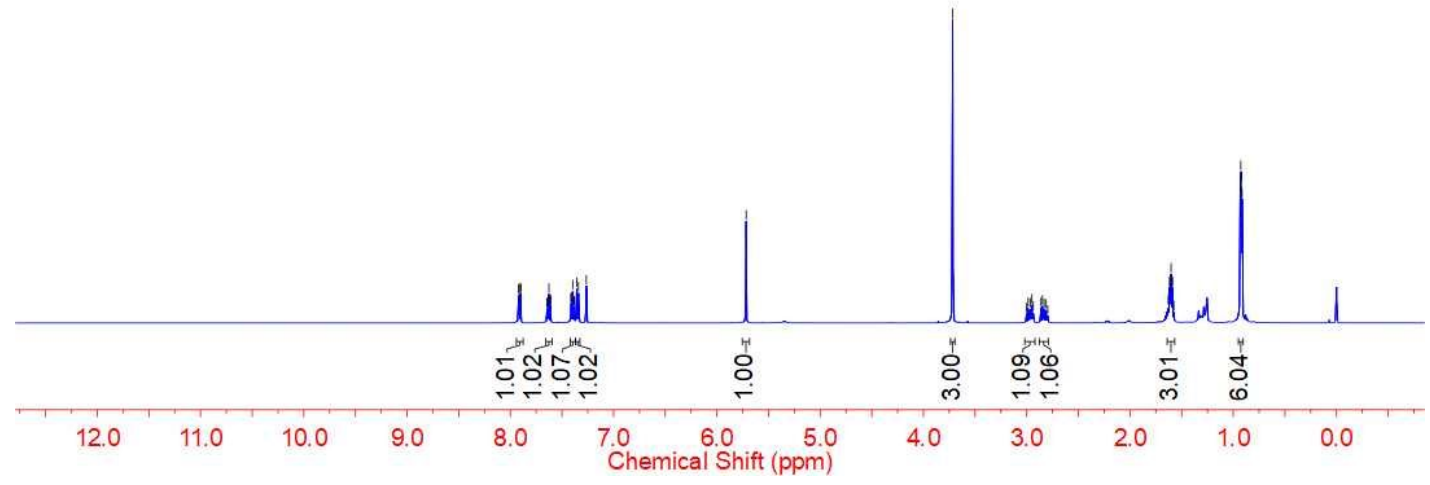

Figure S7. ${ }^{1} \mathrm{H}$ NMR (500 $\left.\mathrm{MHz}, \mathrm{CDCl}_{3}\right)$ spectrum of $3 d$.

\begin{tabular}{|c|c|c|c|c|c|}
\hline & 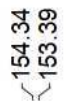 & 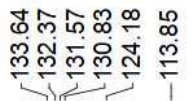 & 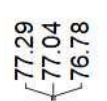 & $\frac{\mathscr{3}}{\infty}$ & 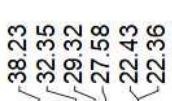 \\
\hline
\end{tabular}<smiles>CC(C)CCC(=O)C(C)(C)c1nc2ccccc2n(C)c1=O</smiles>

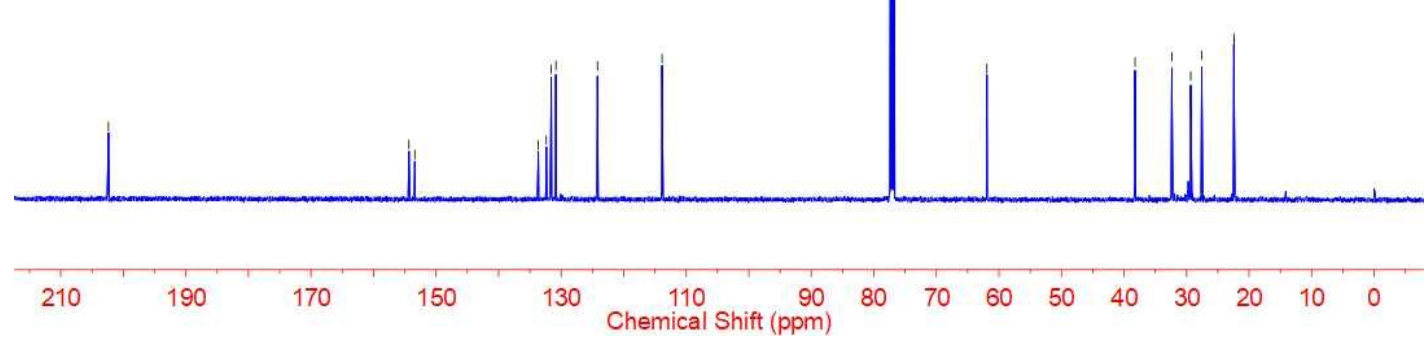

Figure S8. ${ }^{13} \mathrm{C}$ NMR (126 MHz, $\left.\mathrm{CDCl}_{3}\right)$ spectrum of $\mathbf{3 d}$. 


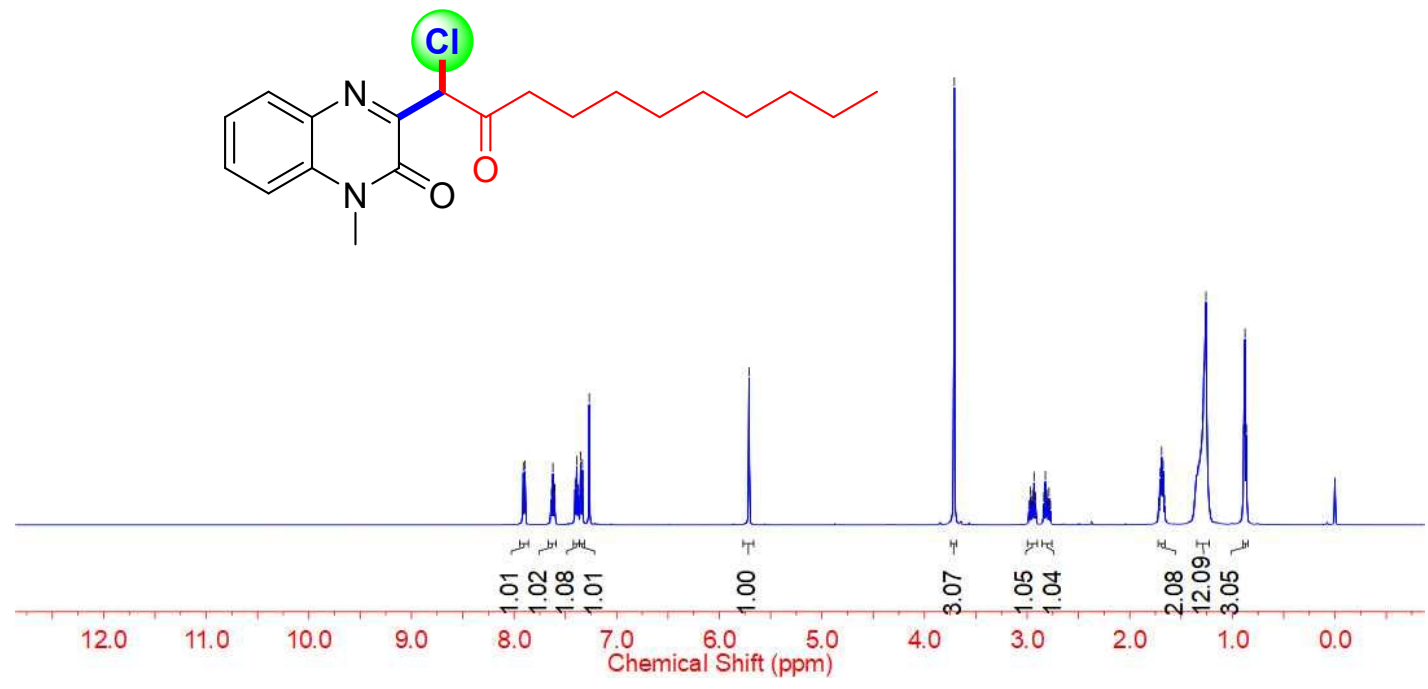

Figure S9. ${ }^{1} \mathrm{H} \mathrm{NMR}\left(500 \mathrm{MHz}, \mathrm{CDCl}_{3}\right)$ spectrum of $3 \mathbf{e}$.

ஸें<smiles>CCCCCCCCCC(=O)C(C)(Cl)c1nc2ccccc2n(C)c1=O</smiles>

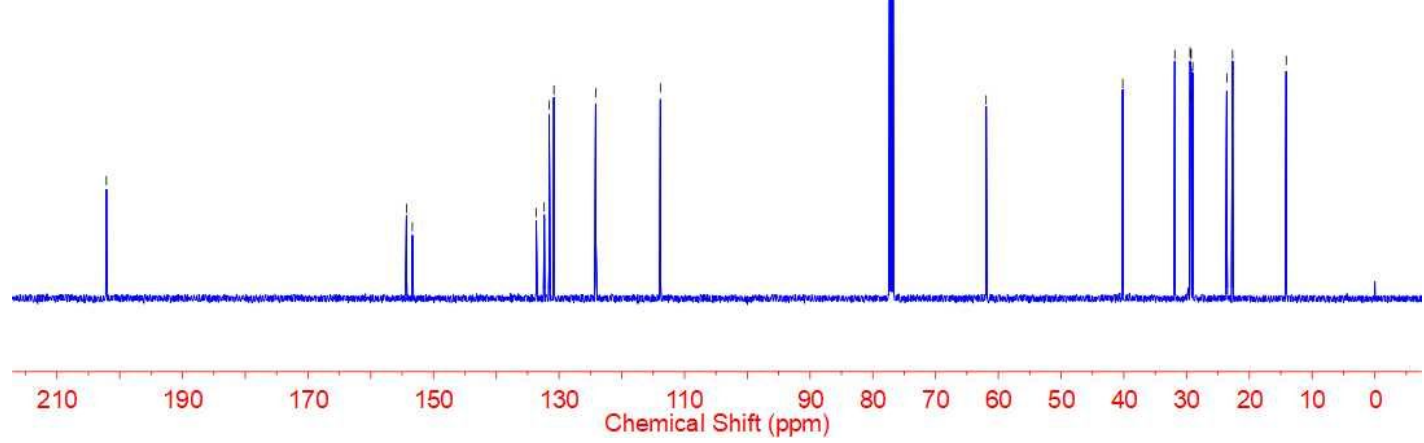

Figure S10. ${ }^{13} \mathrm{C}$ NMR $\left(126 \mathrm{MHz}, \mathrm{CDCl}_{3}\right)$ spectrum of $3 \mathbf{e}$. 
<smiles>Cn1c(=O)c(C(Cl)(Cl)C(=O)C2CC2)nc2ccccc21</smiles>

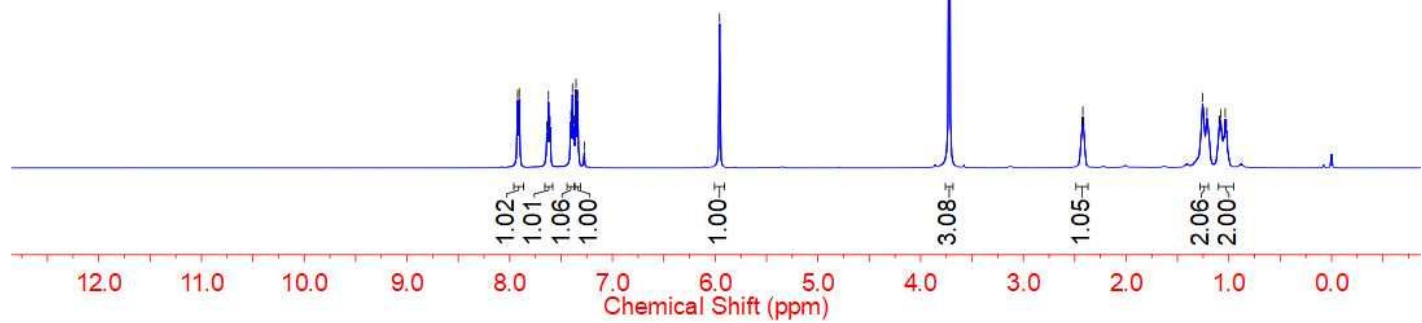

Figure S11. ${ }^{1} \mathrm{H}$ NMR $\left(500 \mathrm{MHz}, \mathrm{CDCl}_{3}\right)$ spectrum of $\mathbf{3 f}$.

ঙุर<smiles>Cn1c(=O)c(C(Cl)(Cl)C(=O)C2CC2)nc2ccccc21</smiles>

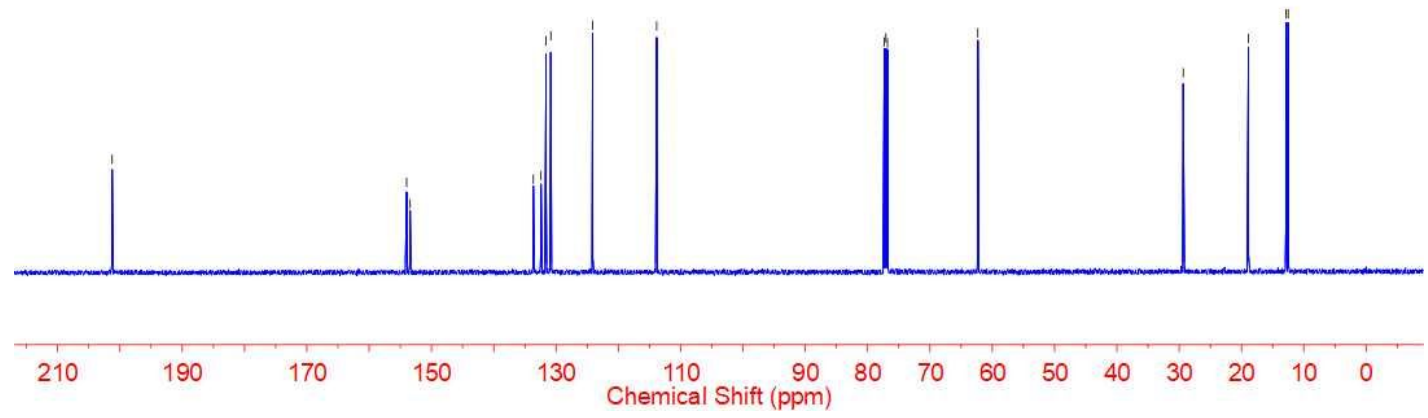

Figure S12. ${ }^{13} \mathrm{C}$ NMR (126 $\left.\mathrm{MHz}, \mathrm{CDCl}_{3}\right)$ spectrum of $\mathbf{3 f}$. 
<smiles>Cn1c(=O)c(C(C)(C)C(=O)C2CCCCC2)nc2ccccc21</smiles>

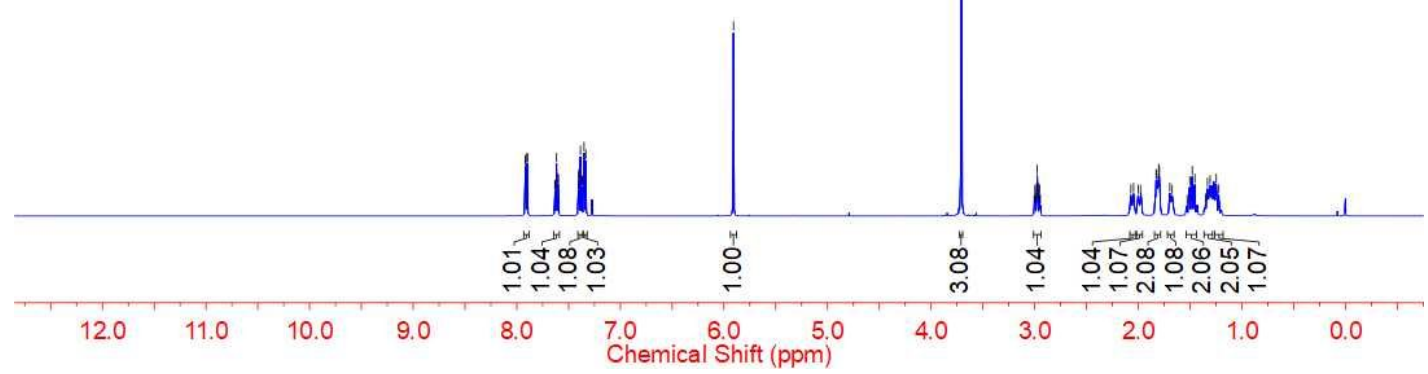

Figure S13. ${ }^{1} \mathrm{H}$ NMR $\left(500 \mathrm{MHz}, \mathrm{CDCl}_{3}\right)$ spectrum of $\mathbf{3 g}$.

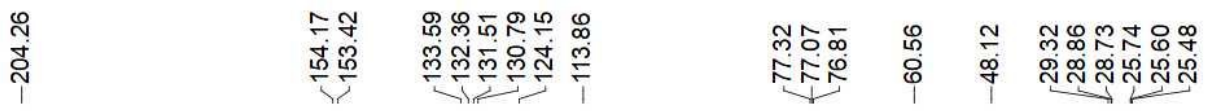<smiles>Cn1c(=O)c(C(C)(Cl)C(=O)C2CCCCC2)nc2ccccc21</smiles>

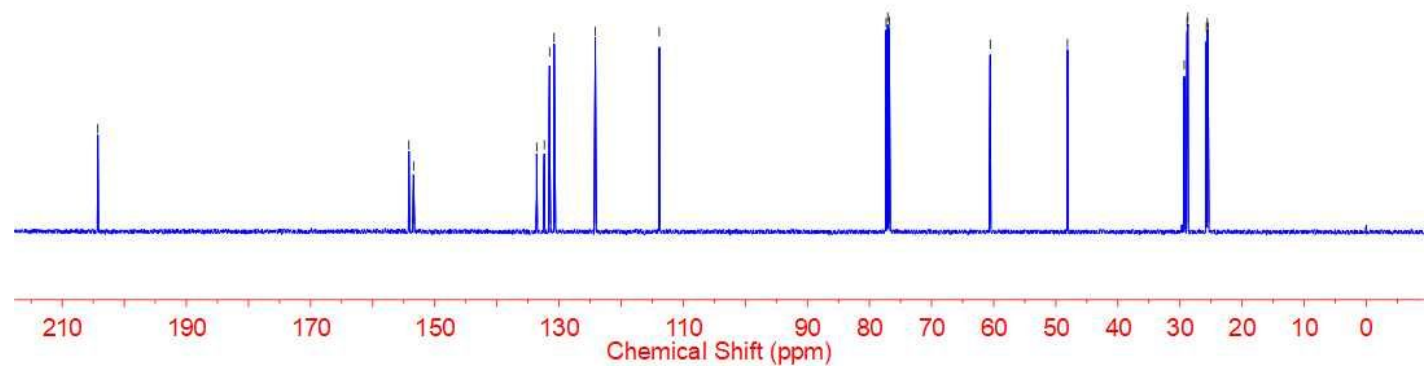

Figure S14. ${ }^{13} \mathrm{C} \mathrm{NMR}\left(126 \mathrm{MHz}, \mathrm{CDCl}_{3}\right)$ spectrum of $\mathbf{3 g}$. 


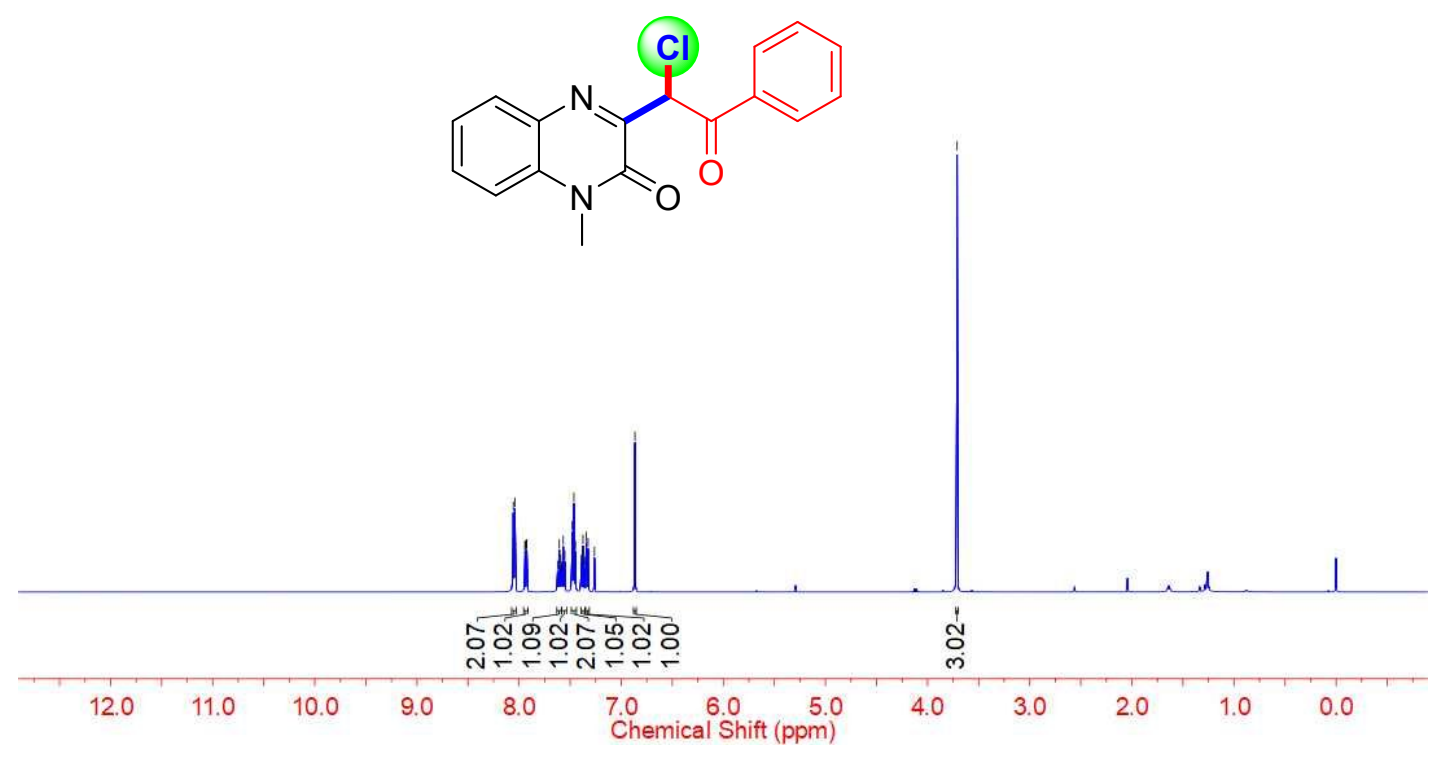

Figure S15. ${ }^{1} \mathrm{H}$ NMR $\left(500 \mathrm{MHz}, \mathrm{CDCl}_{3}\right)$ spectrum of $\mathbf{3 h}$.

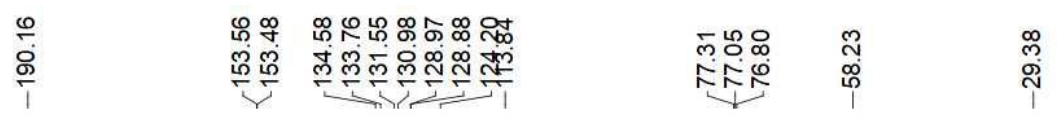<smiles>Cn1c(=O)c(C(Cl)(Cl)C(=O)c2ccccc2)nc2ccccc21</smiles>

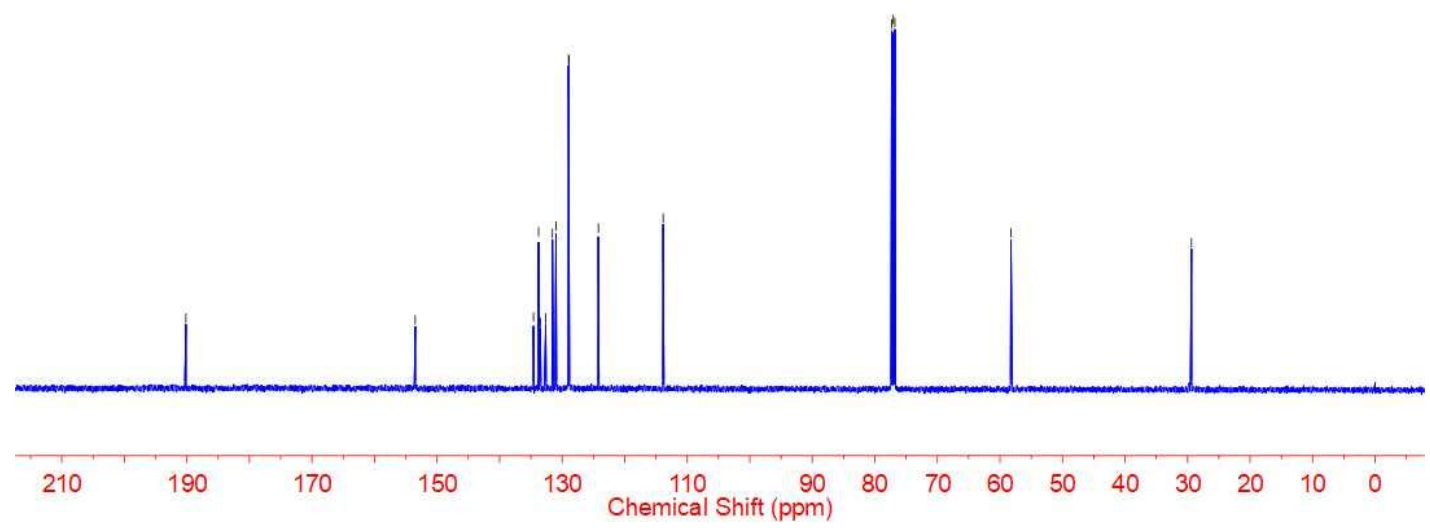

Figure S16. ${ }^{13} \mathrm{C}$ NMR $\left(126 \mathrm{MHz}, \mathrm{CDCl}_{3}\right)$ spectrum of $\mathbf{3 h}$. 
<smiles>Cn1c(=O)c(C(Cl)(Cl)C(=O)c2cccc(Br)c2)nc2ccccc21</smiles>

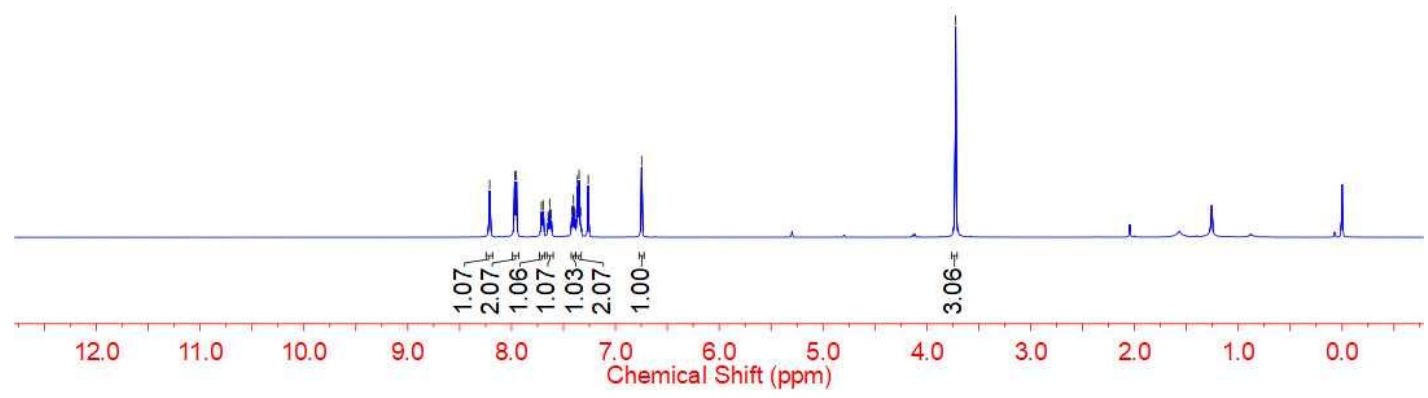

Figure S17. ${ }^{1} \mathrm{H}$ NMR $\left(500 \mathrm{MHz}, \mathrm{CDCl}_{3}\right)$ spectrum of $3 \mathbf{i}$.

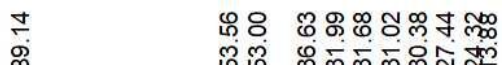<smiles>Cn1c(=O)c(C(Cl)(Cl)C(=O)c2cccc(Br)c2)nc2ccccc21</smiles>

Figure S18. ${ }^{13} \mathrm{C}$ NMR $\left(126 \mathrm{MHz}, \mathrm{CDCl}_{3}\right)$ spectrum of $3 \mathbf{i}$. 


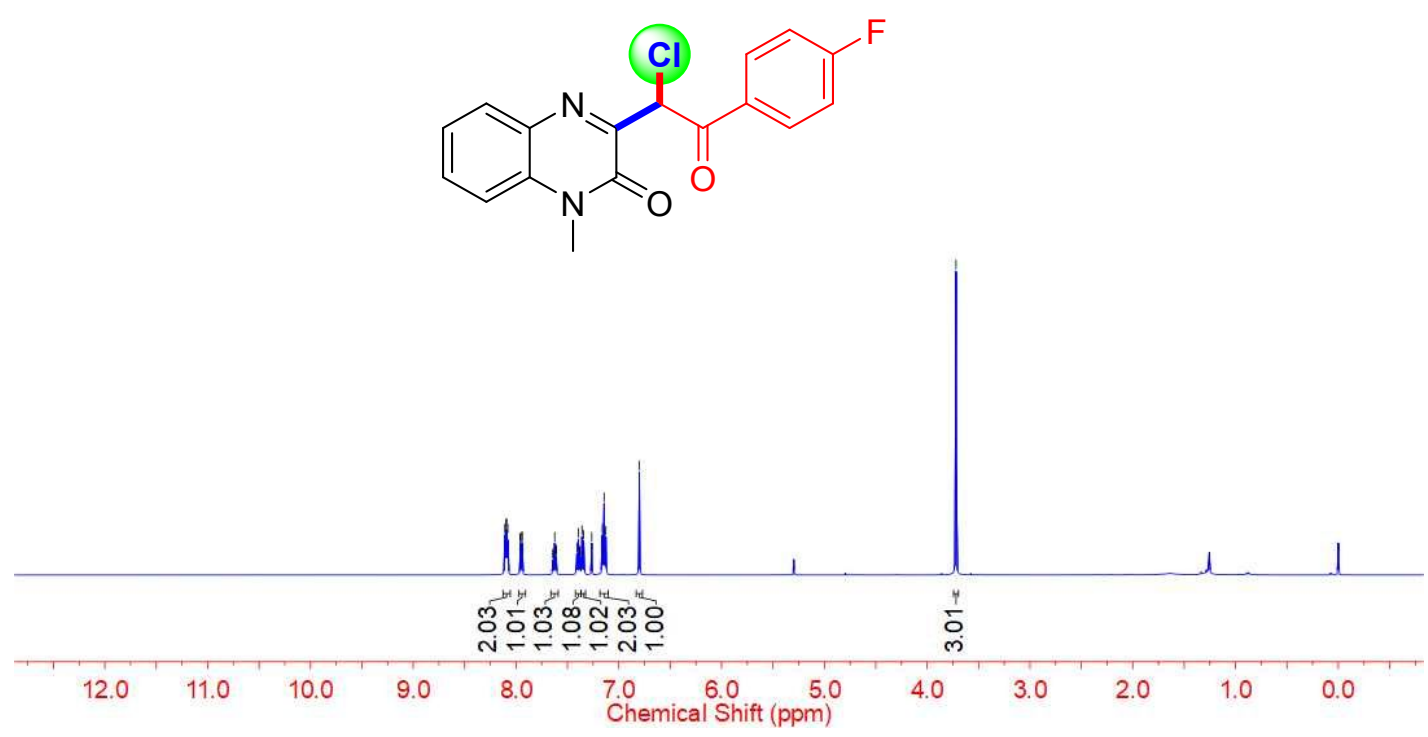

Figure S19. ${ }^{1} \mathrm{H}$ NMR $\left(500 \mathrm{MHz}, \mathrm{CDCl}_{3}\right)$ spectrum of $\mathbf{3 j}$.

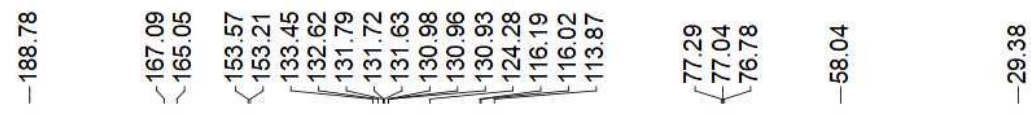

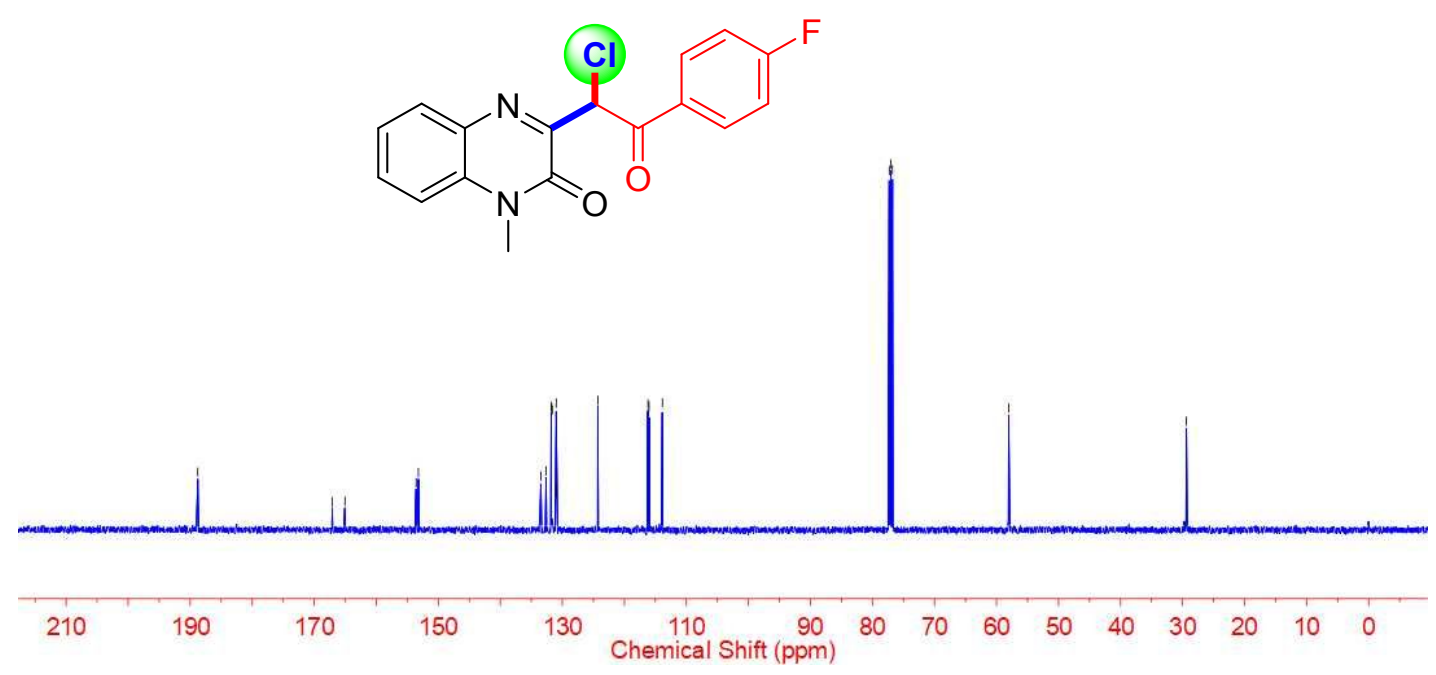

Figure S20. ${ }^{13} \mathrm{C}$ NMR $\left(126 \mathrm{MHz}, \mathrm{CDCl}_{3}\right)$ spectrum of $\mathbf{3 j}$. 

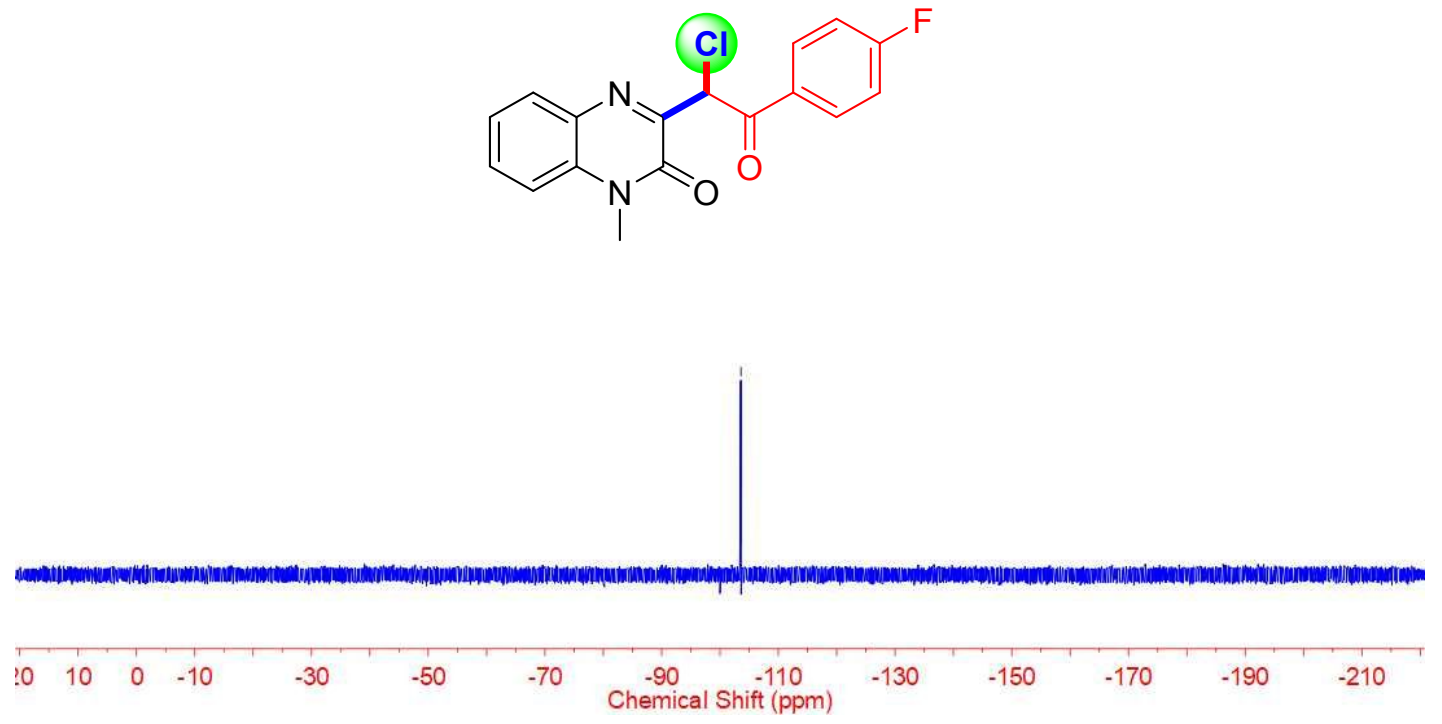

Figure S21. ${ }^{19} \mathrm{~F}$ NMR (471 MHz, $\left.\mathrm{CDCl}_{3}\right)$ spectrum of $\mathbf{3 j}$.<smiles>Cn1c(=O)c(C(Cl)(Cl)C(=O)c2ccco2)nc2ccccc21</smiles>

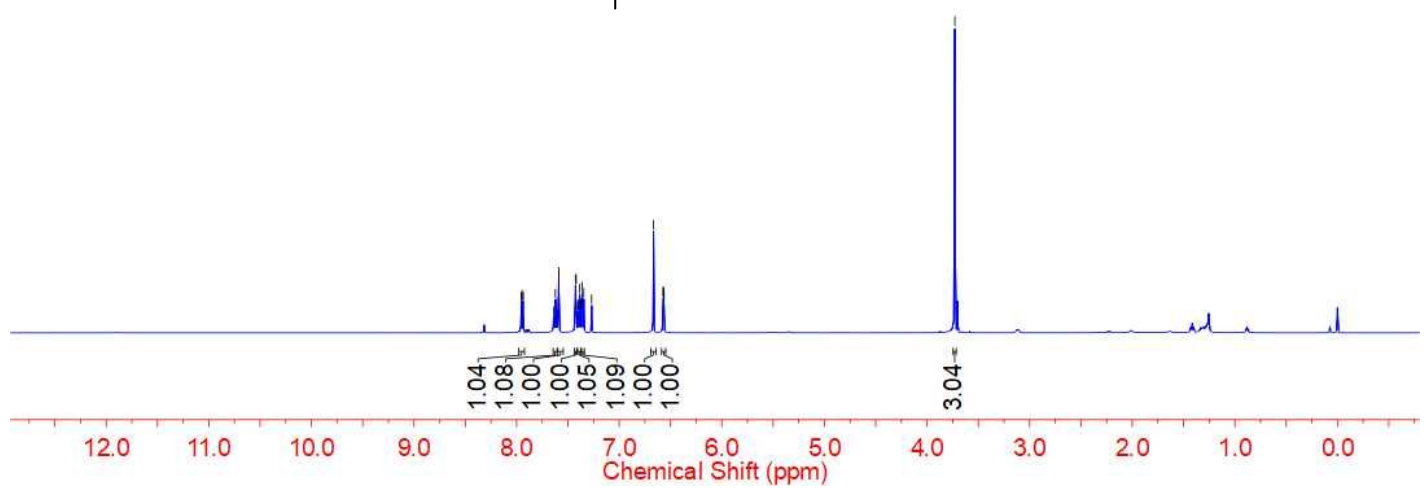

Figure S22. ${ }^{1} \mathrm{H}$ NMR $\left(500 \mathrm{MHz}, \mathrm{CDCl}_{3}\right)$ spectrum of $\mathbf{3 k}$. 
<smiles>Cn1c(=O)c(C(Cl)(Cl)C(=O)c2ccco2)nc2ccccc21</smiles>
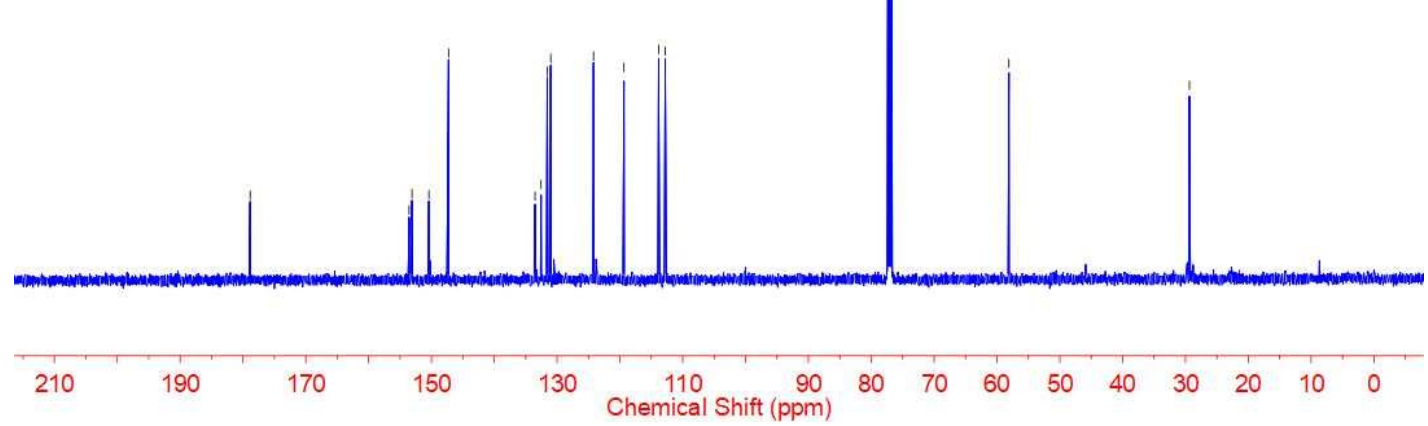

Figure S23. ${ }^{13} \mathrm{C}$ NMR $\left(126 \mathrm{MHz}, \mathrm{CDCl}_{3}\right)$ spectrum of 3k.<smiles>Cn1c(=O)c(C(Cl)(Cl)C(=O)c2ccsc2)nc2ccccc21</smiles>

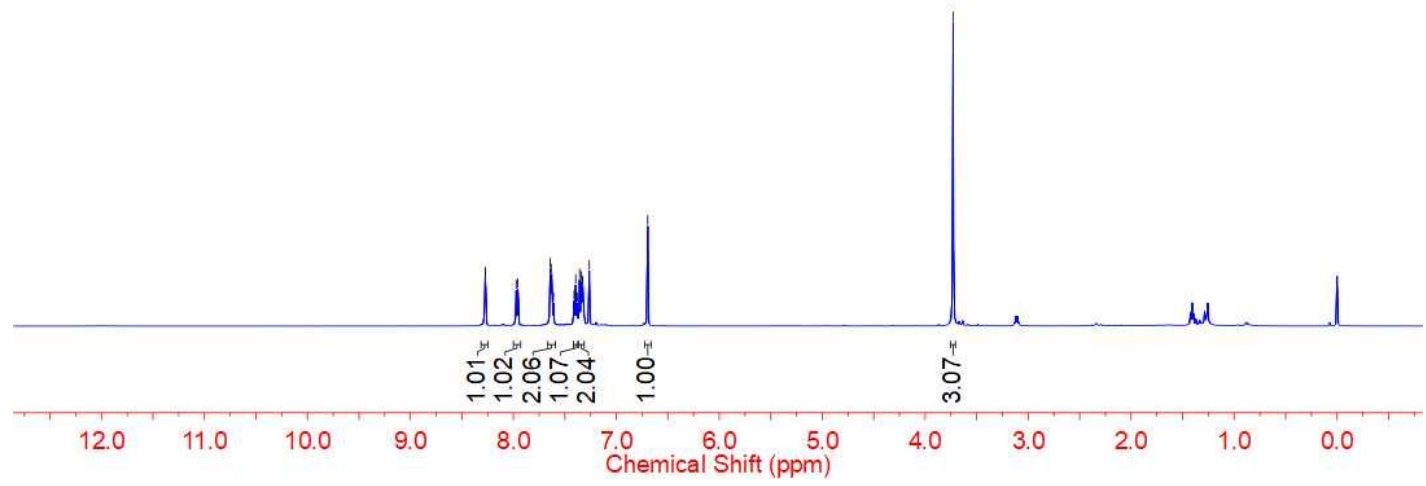

Figure S24. ${ }^{1} \mathrm{H}$ NMR $\left(500 \mathrm{MHz}, \mathrm{CDCl}_{3}\right)$ spectrum of 31. 


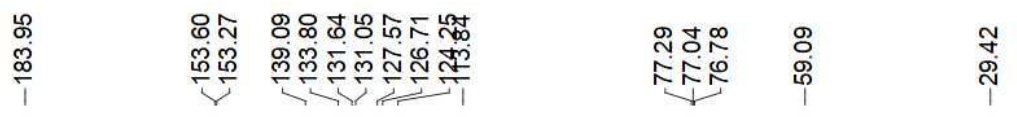<smiles>Cn1c(=O)c(C(Cl)(Cl)C(=O)c2ccsc2)nc2ccccc21</smiles>
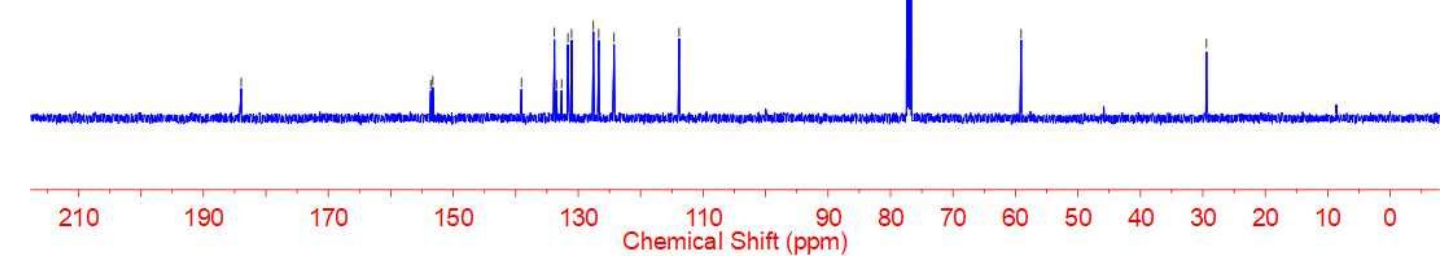

Figure S25. ${ }^{13} \mathrm{C}$ NMR $\left(126 \mathrm{MHz}, \mathrm{CDCl}_{3}\right)$ spectrum of $\mathbf{3 I}$.

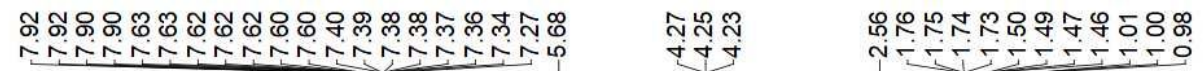

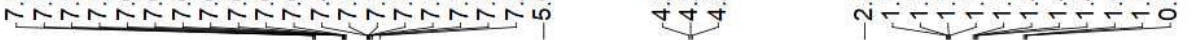<smiles>CCCCn1c(=O)c(C(Cl)(Cl)C(C)=O)nc2ccccc21</smiles>
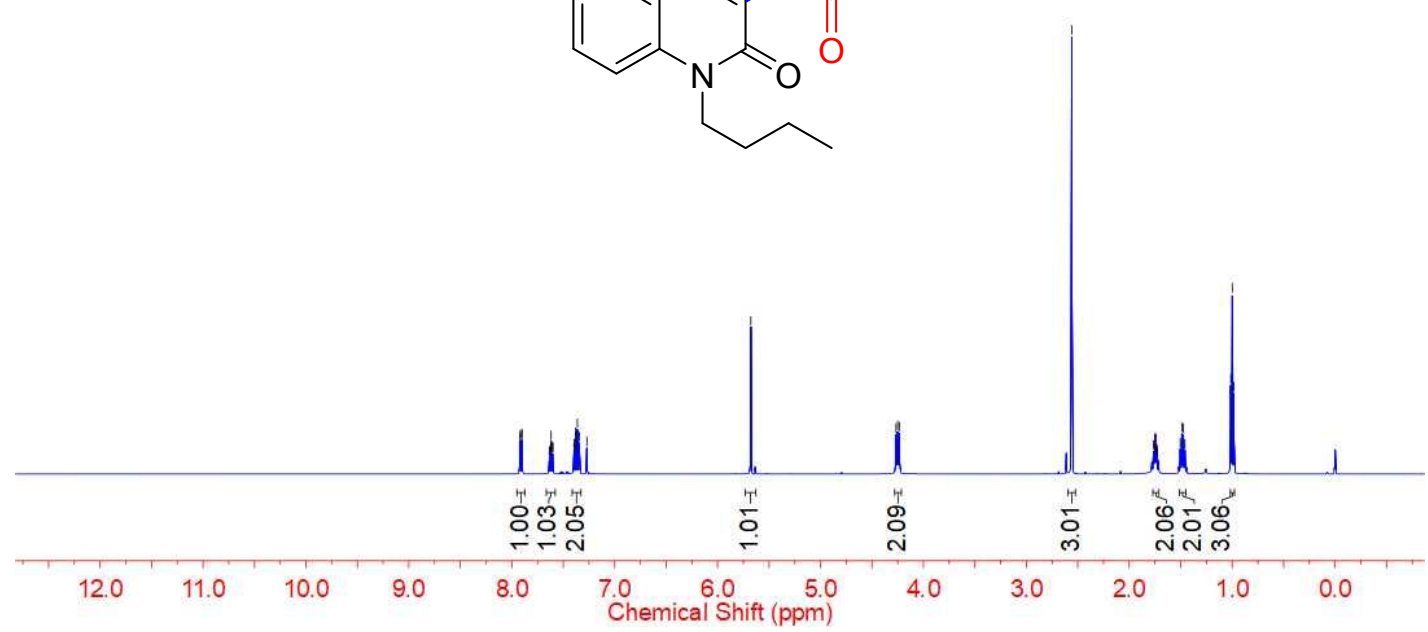

Figure S26. ${ }^{1} \mathrm{H}$ NMR ( $\left.500 \mathrm{MHz}, \mathrm{CDCl}_{3}\right)$ spectrum of $\mathbf{3 m}$. 


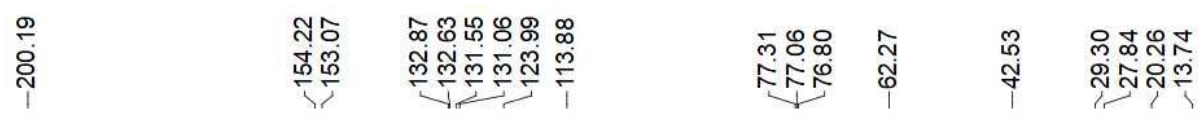

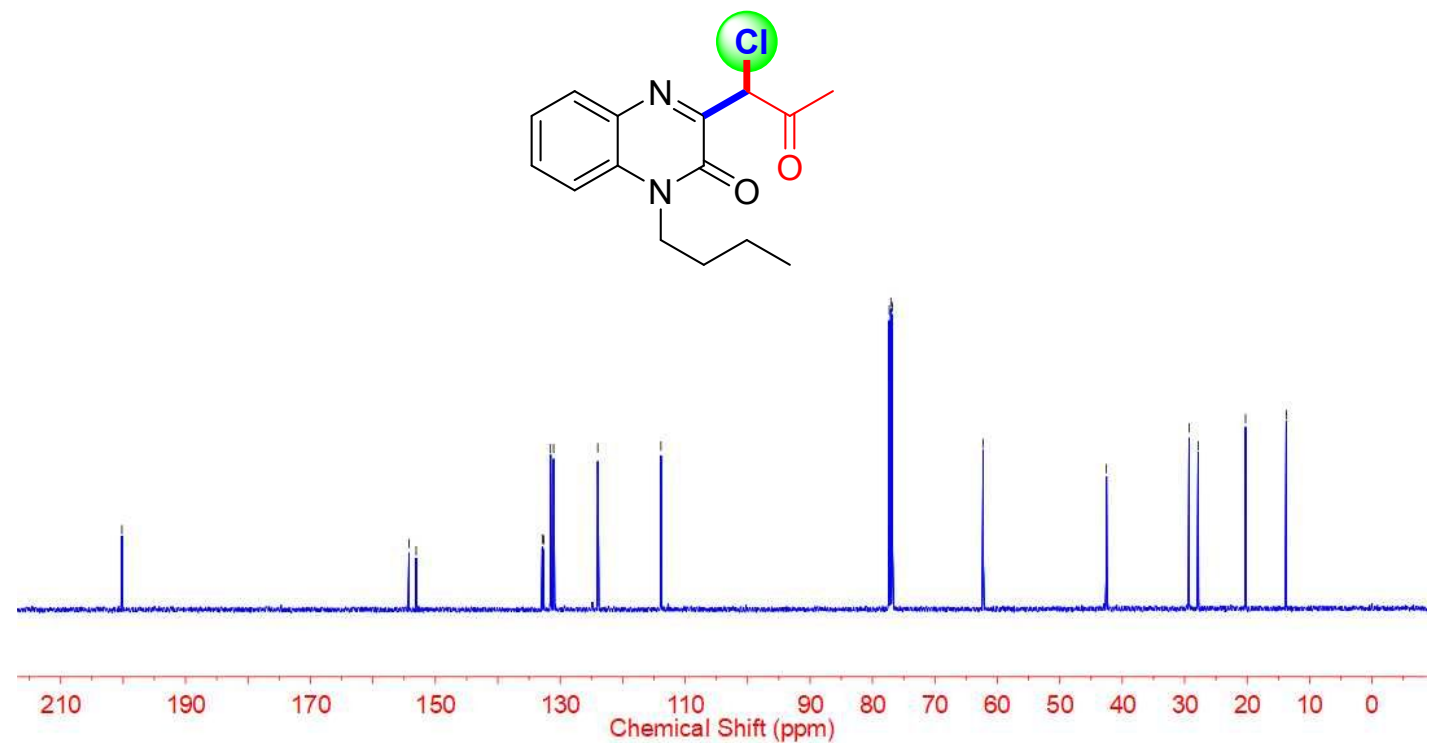

Figure S27. ${ }^{13} \mathrm{C}$ NMR $\left(126 \mathrm{MHz}, \mathrm{CDCl}_{3}\right)$ spectrum of $\mathbf{3 m}$.

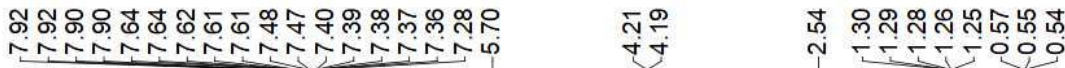

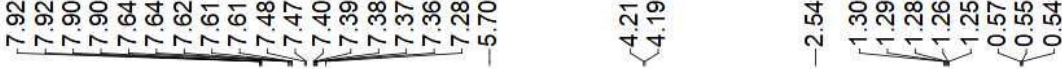

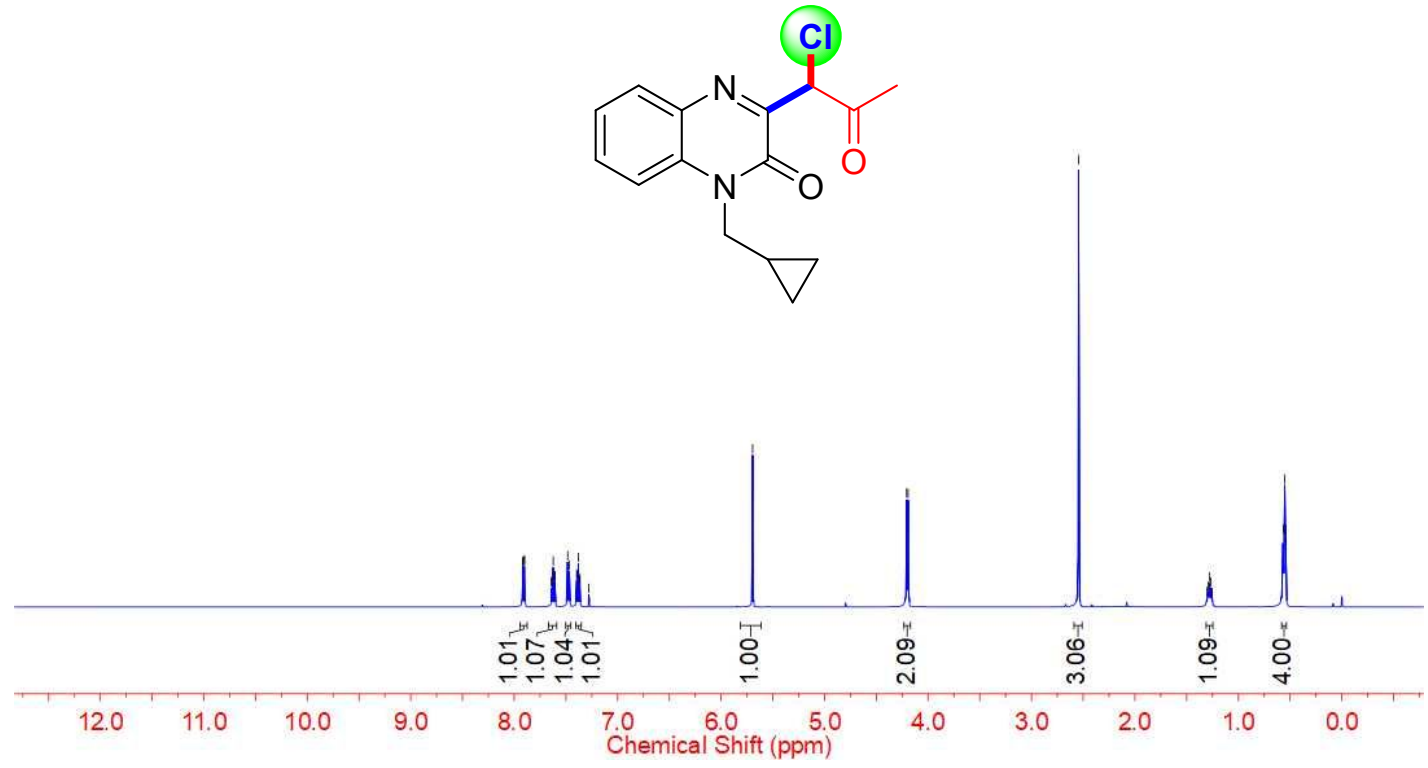

Figure S28. ${ }^{1} \mathrm{H}$ NMR $\left(500 \mathrm{MHz}, \mathrm{CDCl}_{3}\right)$ spectrum of $\mathbf{3 n}$. 


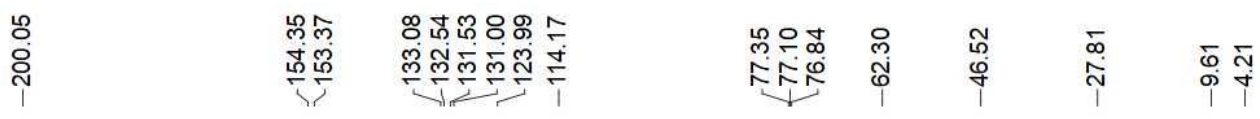

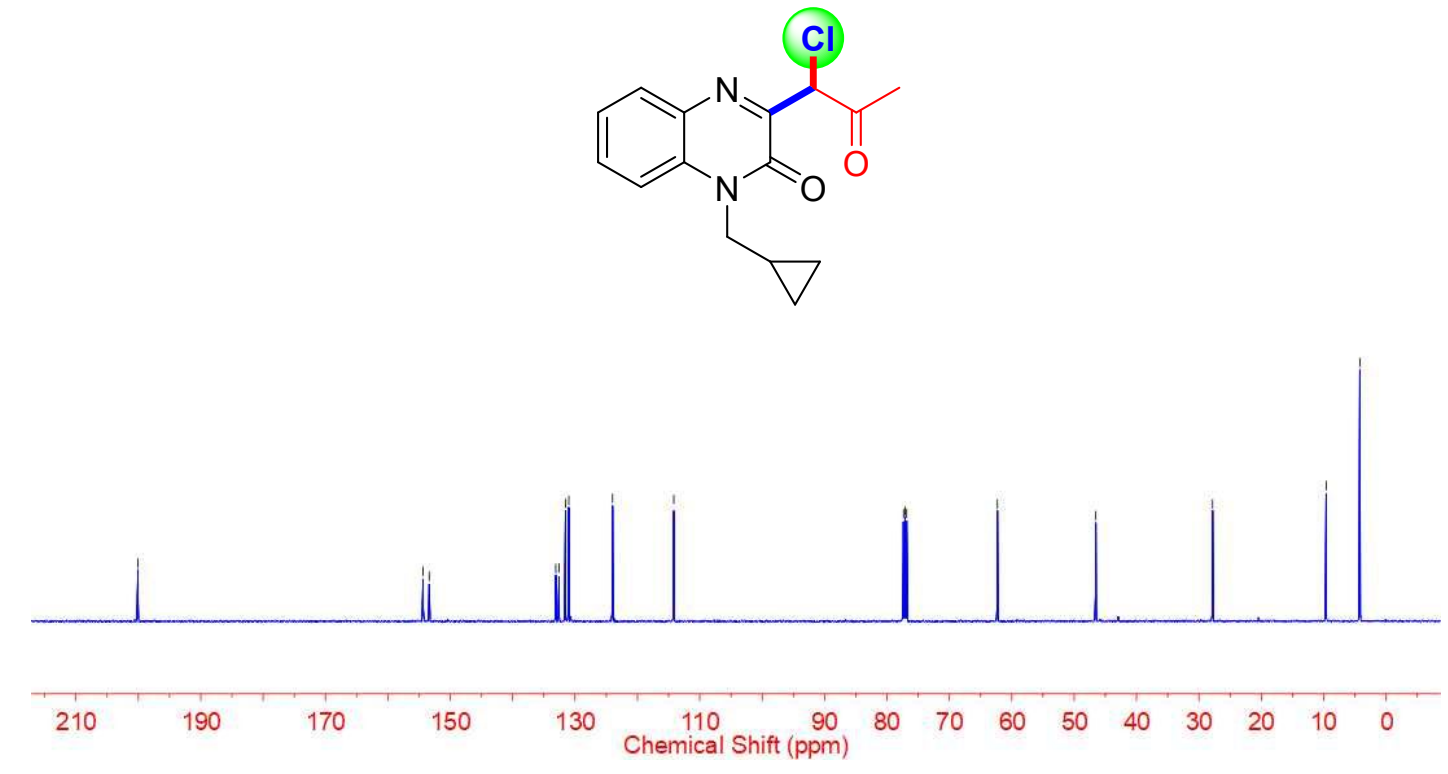

Figure S29. ${ }^{13} \mathrm{C} \mathrm{NMR}\left(126 \mathrm{MHz}, \mathrm{CDCl}_{3}\right)$ spectrum of $\mathbf{3 n}$.

ת<smiles>COC(=O)Cn1c(=O)c(C(C)(C)C(C)=O)nc2ccccc21</smiles>

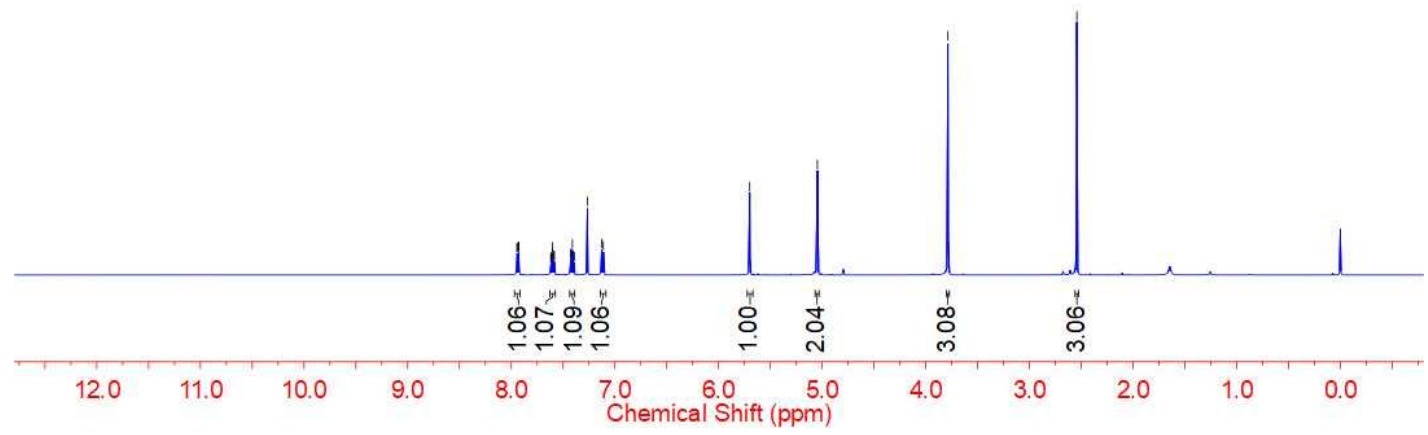

Figure S30. ${ }^{1} \mathrm{H}$ NMR $\left(500 \mathrm{MHz}, \mathrm{CDCl}_{3}\right)$ spectrum of $3 \mathbf{0 .}$ 


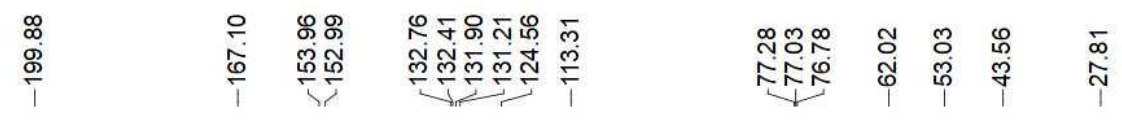

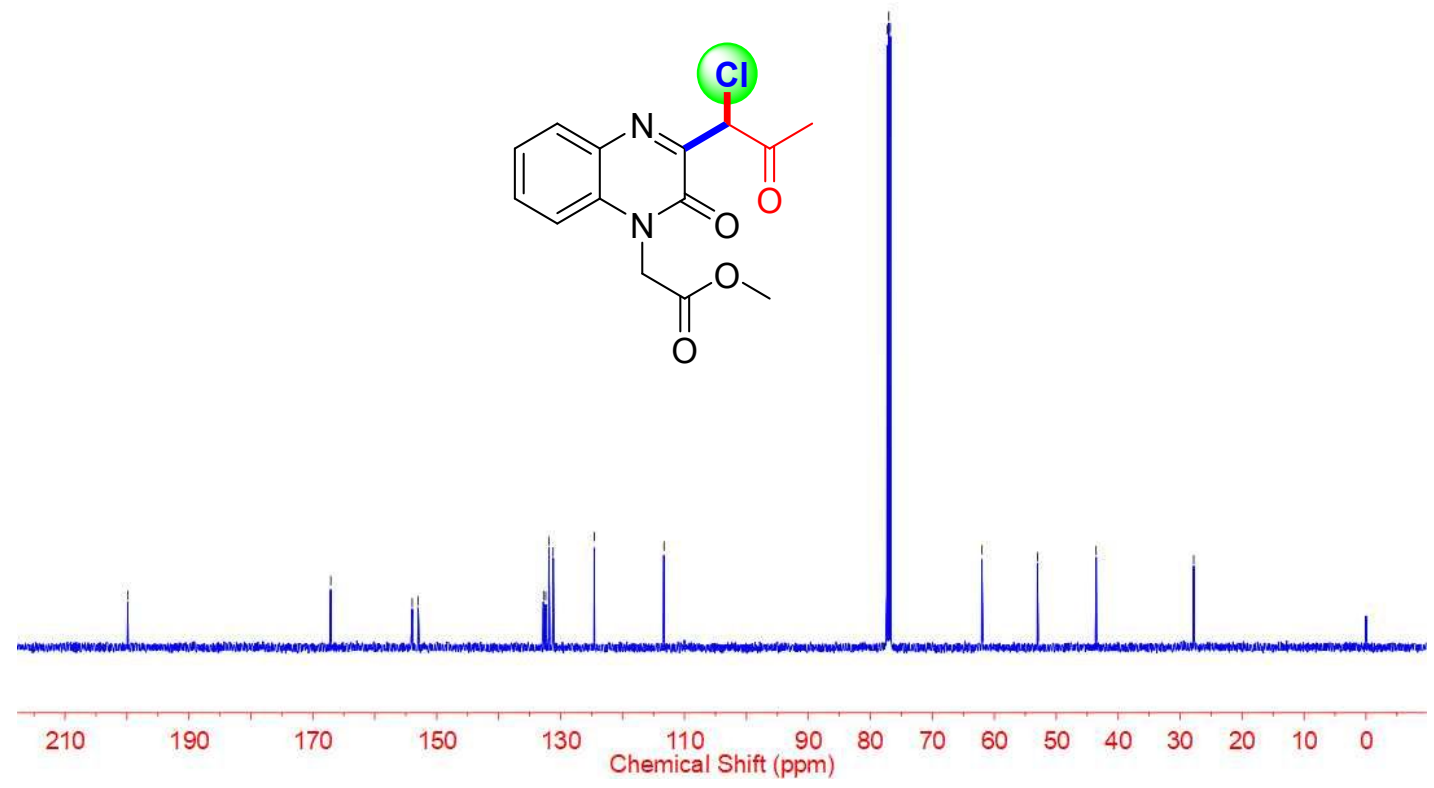

Figure S31. ${ }^{13} \mathrm{C}$ NMR $\left(126 \mathrm{MHz}, \mathrm{CDCl}_{3}\right)$ spectrum of $\mathbf{3 o}$.

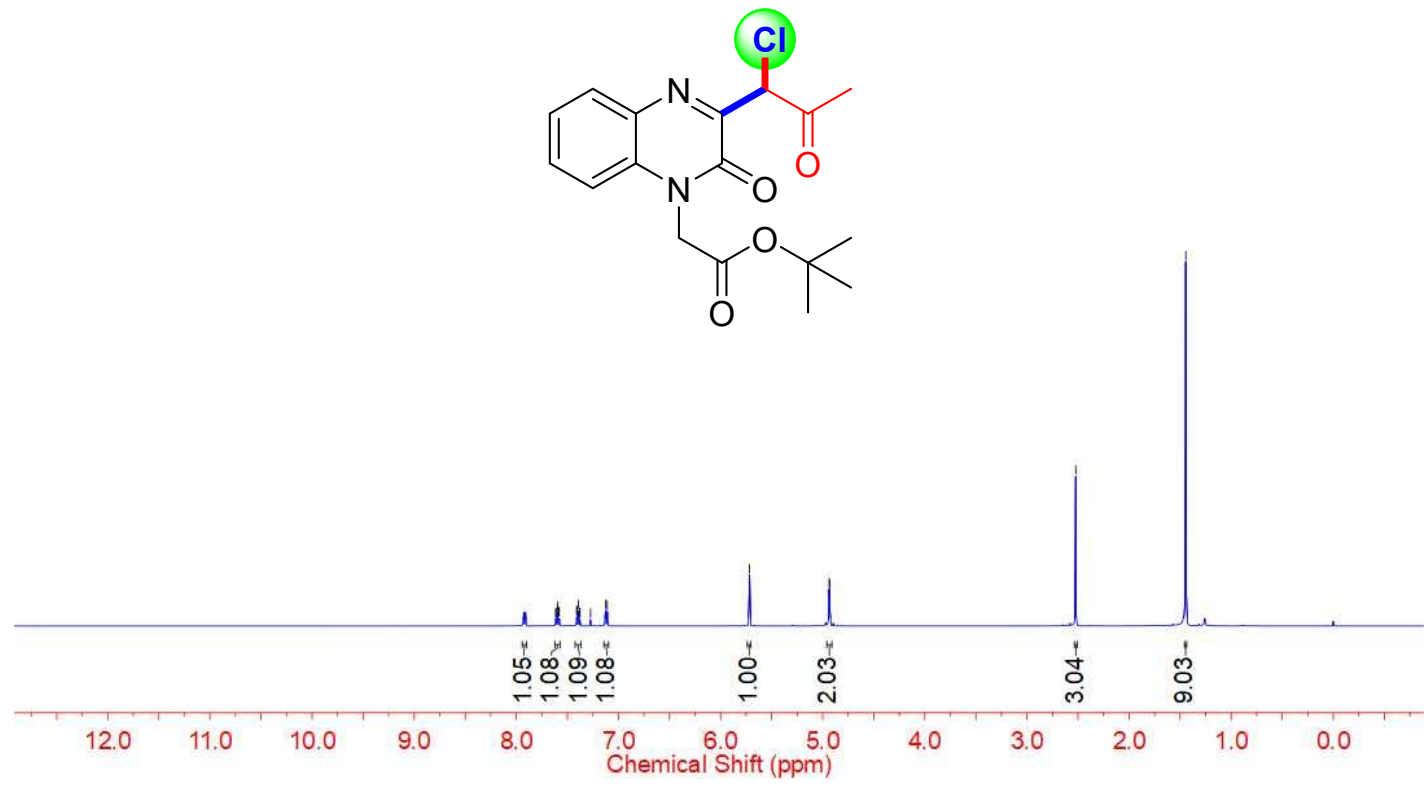

Figure S32. ${ }^{1} \mathrm{H}$ NMR $\left(500 \mathrm{MHz}, \mathrm{CDCl}_{3}\right)$ spectrum of 3p. 


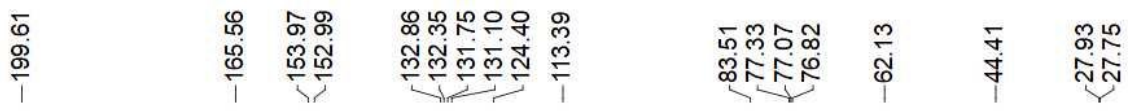

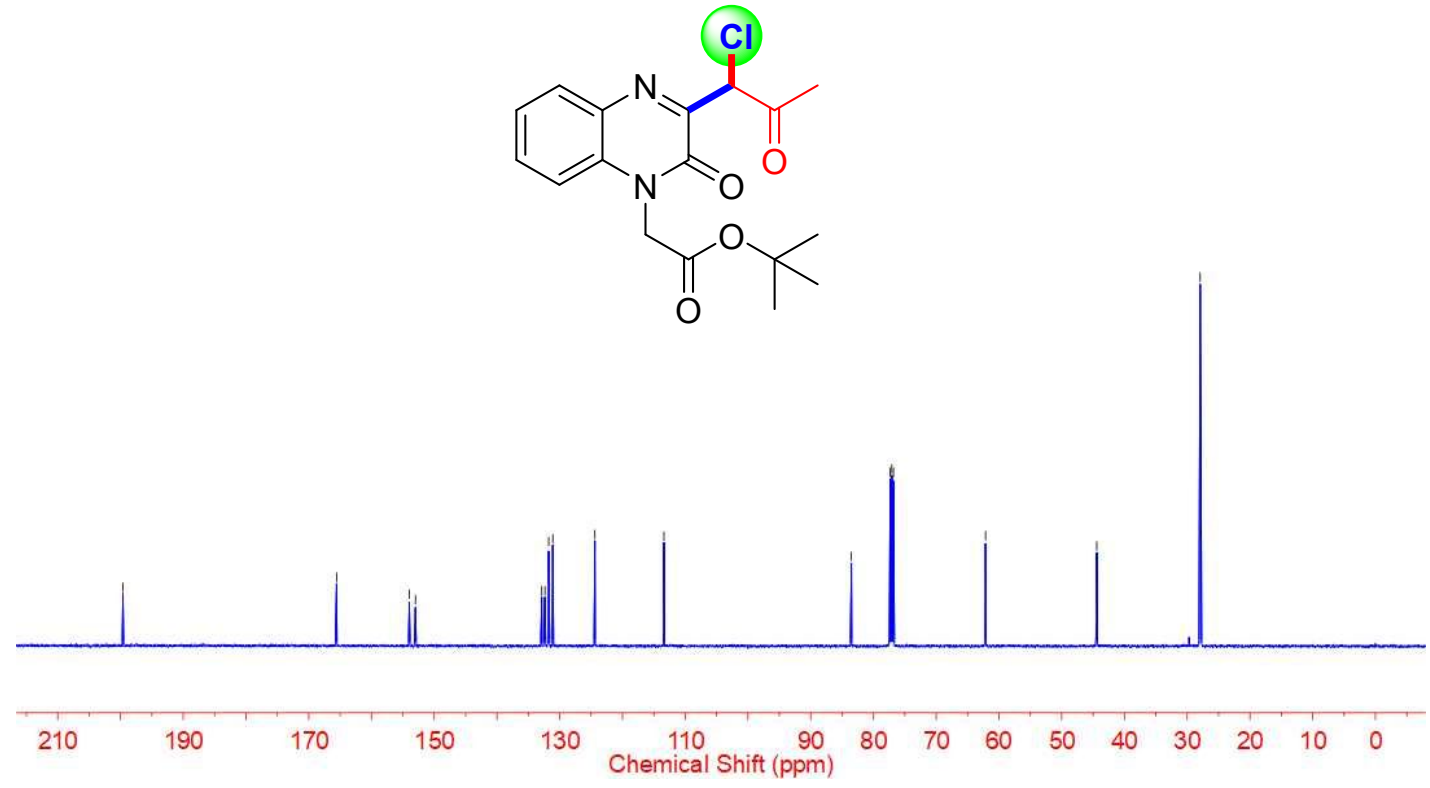

Figure S33. ${ }^{13} \mathrm{C}$ NMR $\left(126 \mathrm{MHz}, \mathrm{CDCl}_{3}\right)$ spectrum of $\mathbf{3 p}$.

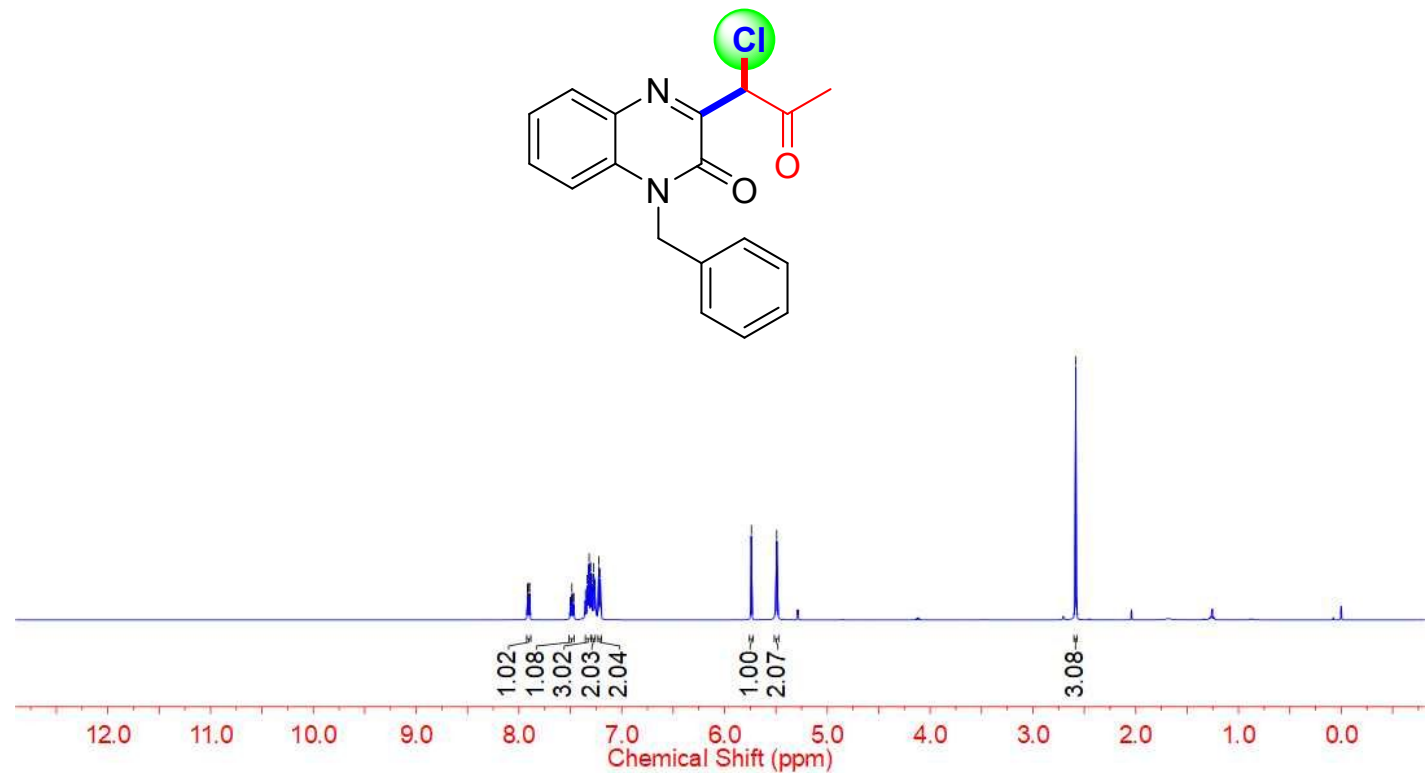

Figure S34. ${ }^{1} \mathrm{H}$ NMR $\left(500 \mathrm{MHz}, \mathrm{CDCl}_{3}\right)$ spectrum of 3q. 


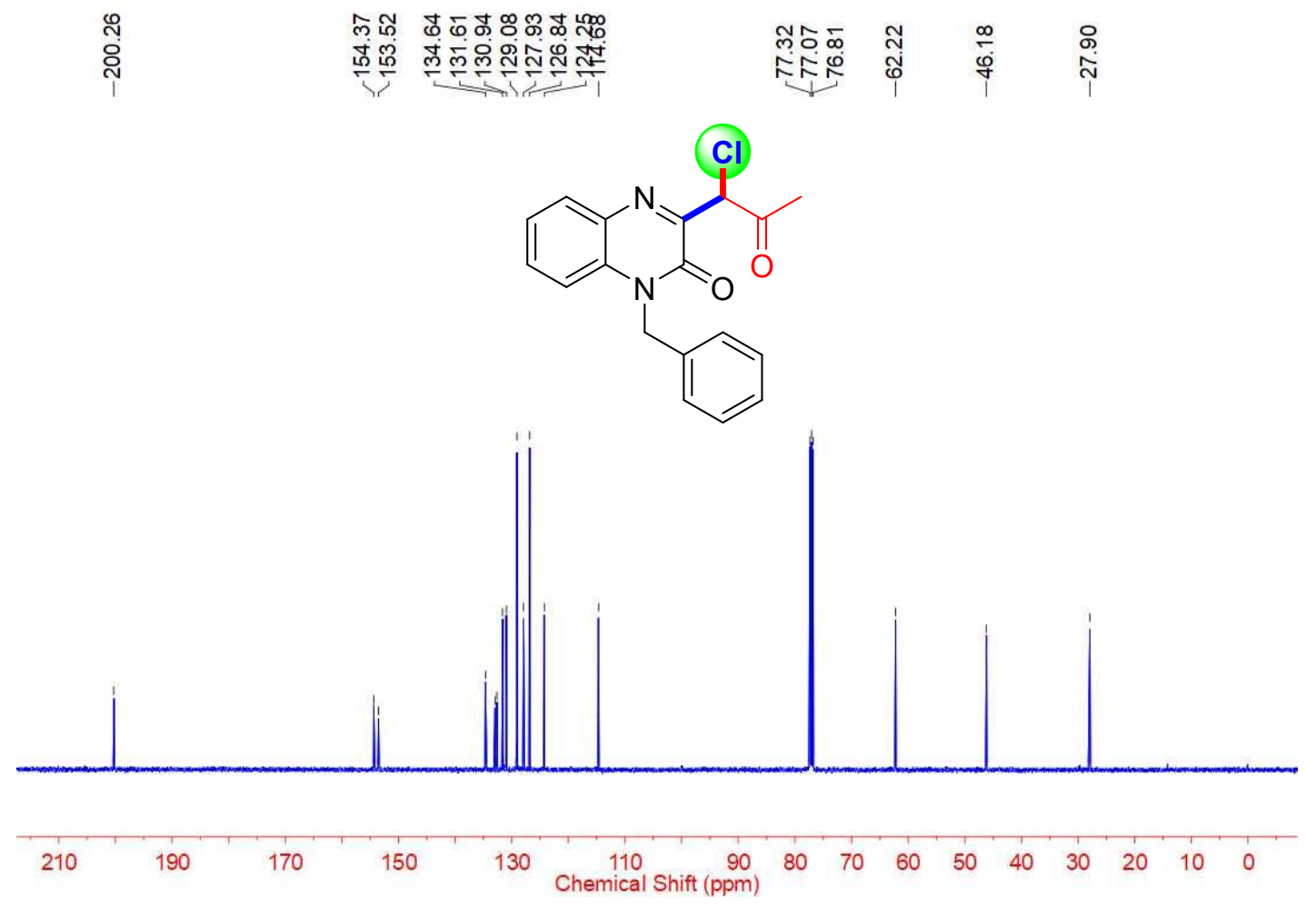

Figure S35. ${ }^{13} \mathrm{C}$ NMR $\left(126 \mathrm{MHz}, \mathrm{CDCl}_{3}\right)$ spectrum of $\mathbf{3 q}$.

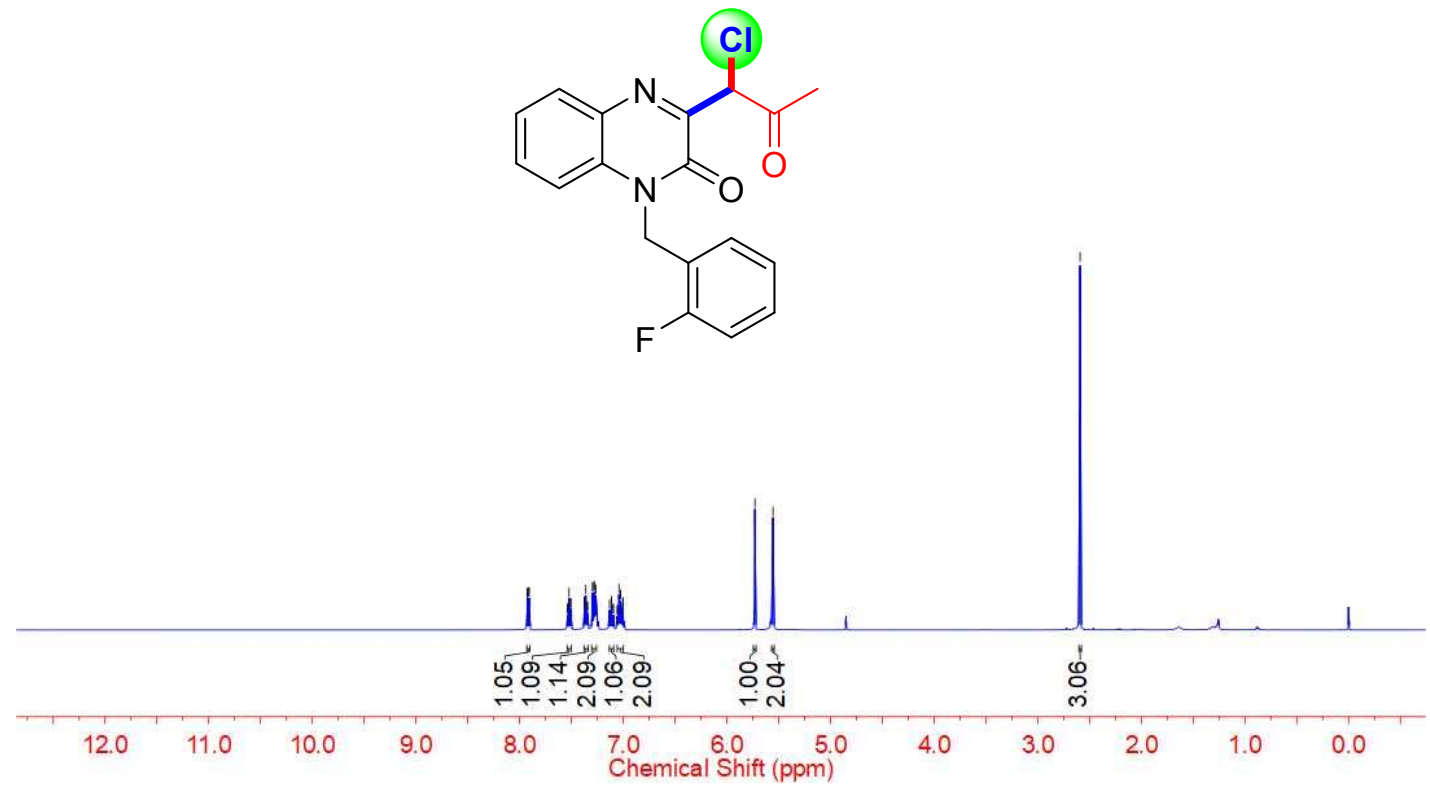

Figure S36. ${ }^{1} \mathrm{H}$ NMR $\left(500 \mathrm{MHz}, \mathrm{CDCl}_{3}\right)$ spectrum of $\mathbf{3 r}$. 


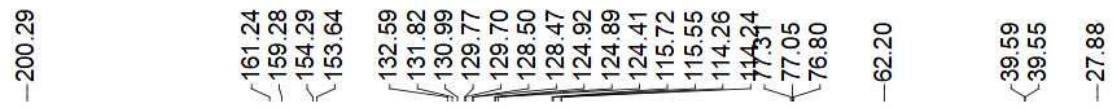

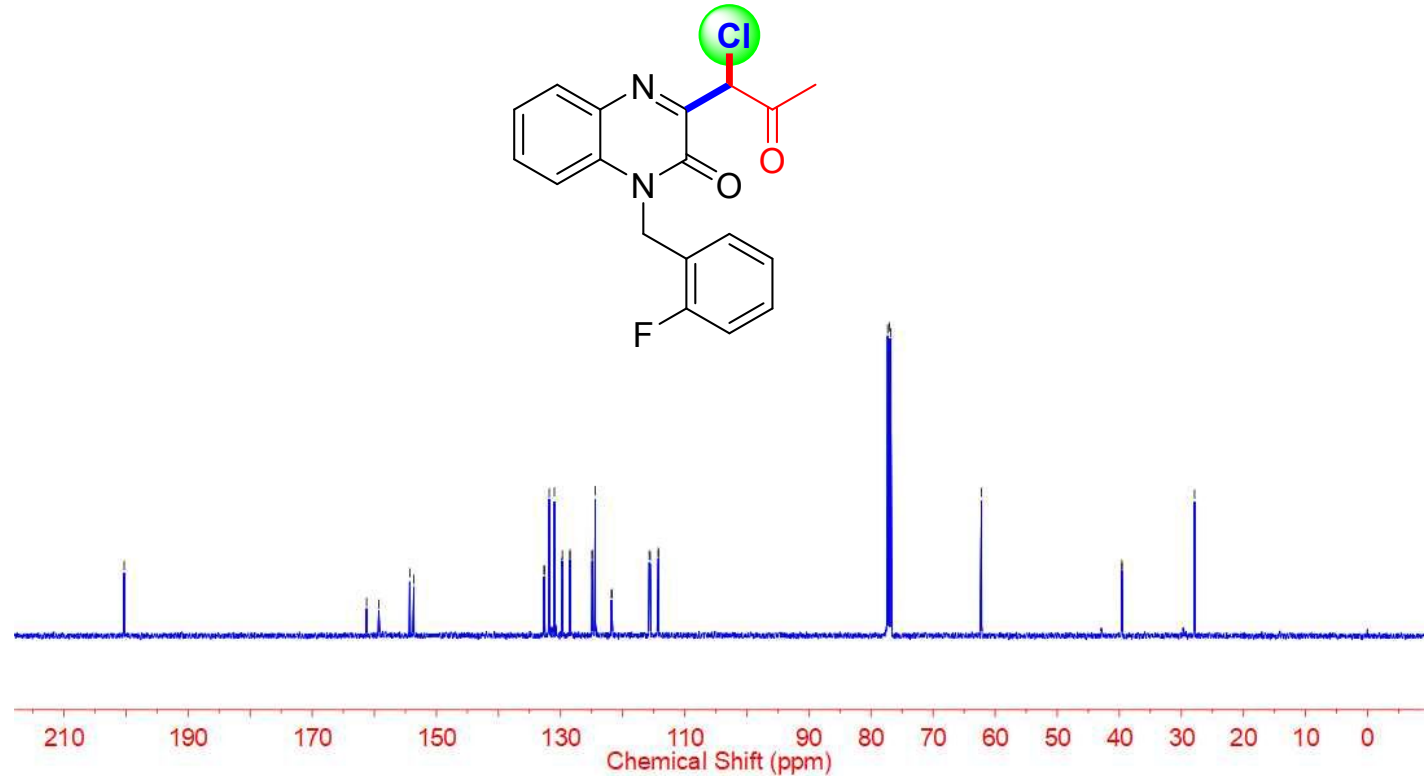

Figure S37. ${ }^{13} \mathrm{C}$ NMR $\left(126 \mathrm{MHz}, \mathrm{CDCl}_{3}\right)$ spectrum of $\mathbf{3 r}$.

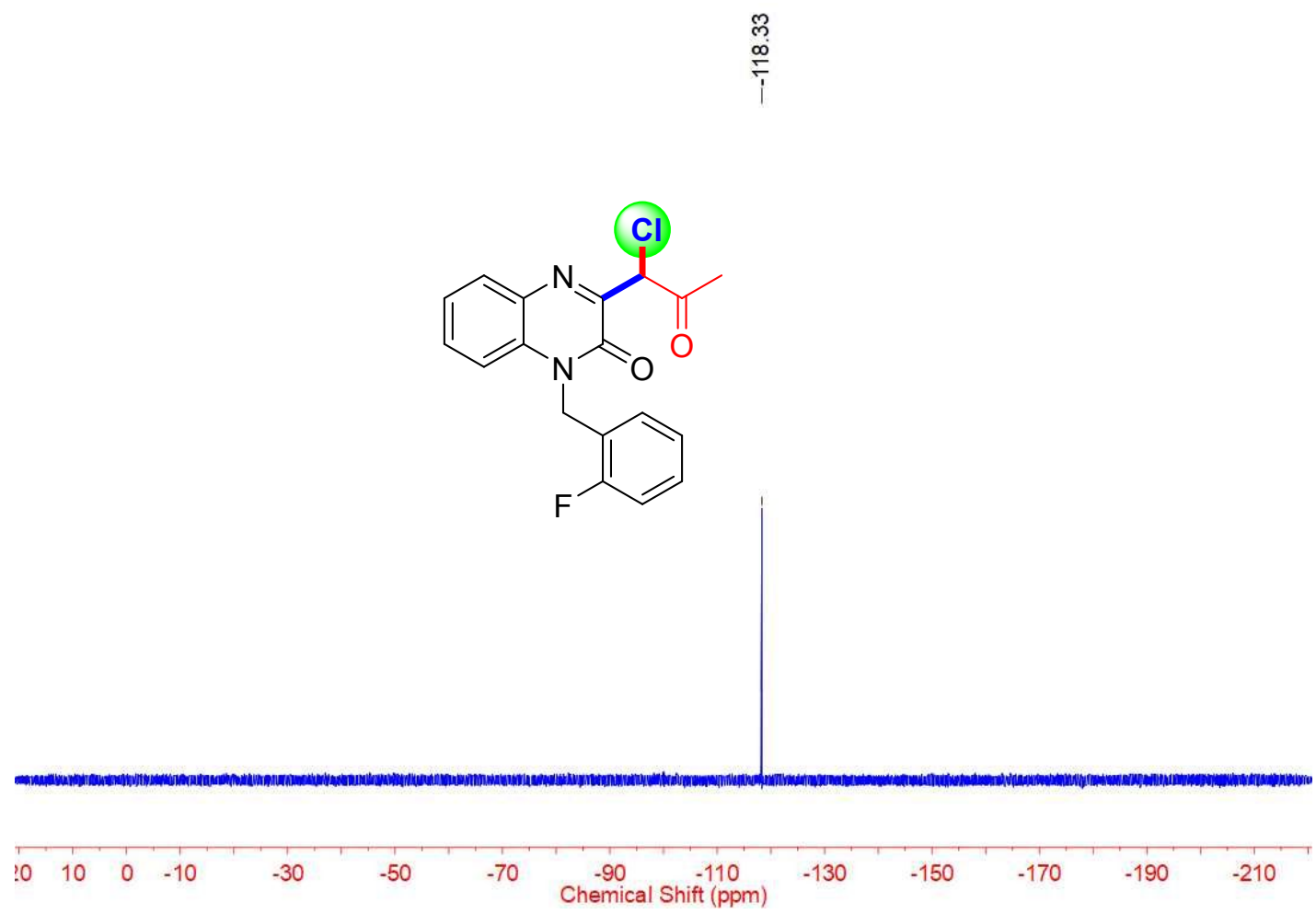

Figure S38. ${ }^{19} \mathrm{~F}$ NMR $\left(471 \mathrm{MHz}, \mathrm{CDCl}_{3}\right)$ spectrum of $\mathbf{3 r}$. 


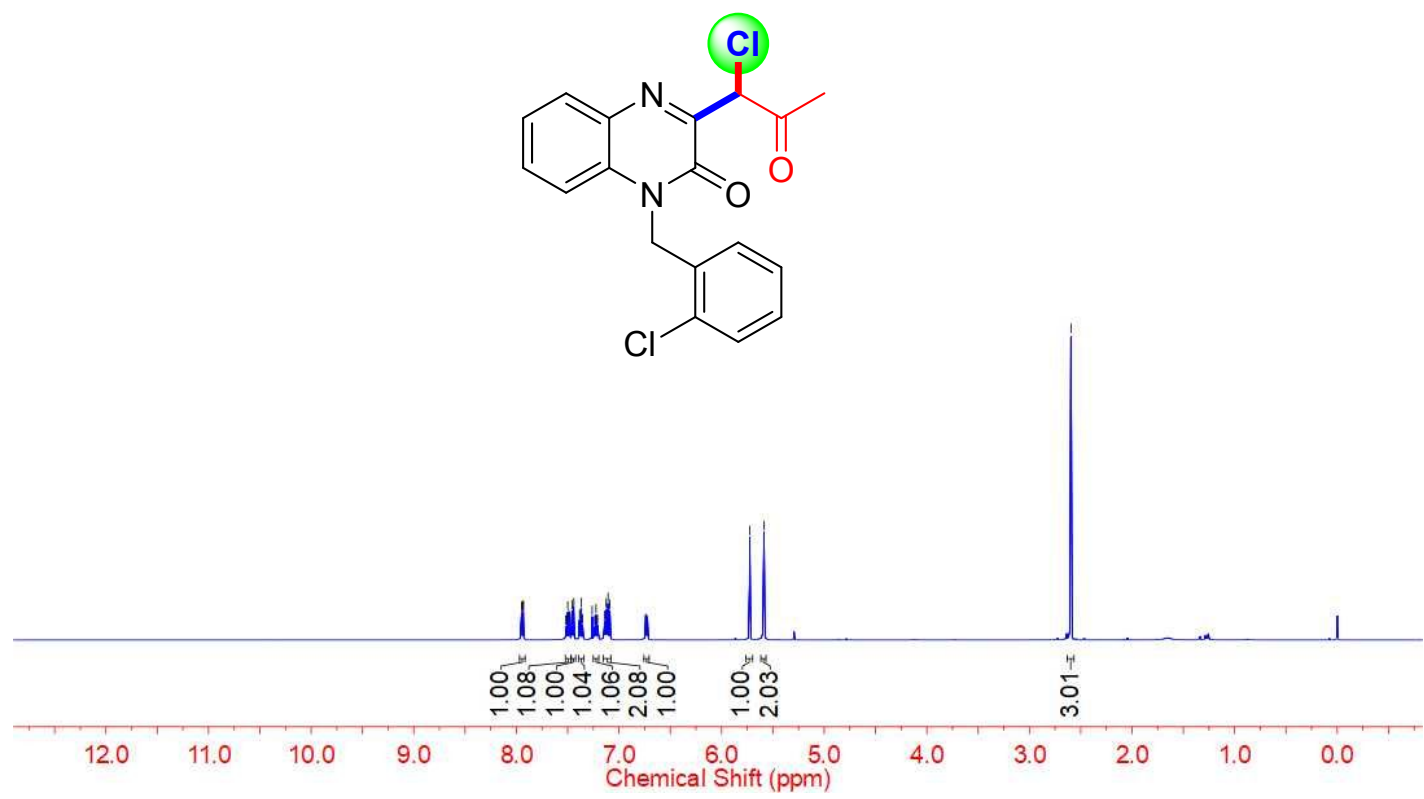

Figure S39. ${ }^{1} \mathrm{H}$ NMR $\left(500 \mathrm{MHz}, \mathrm{CDCl}_{3}\right)$ spectrum of $\mathbf{3 s}$.

वें

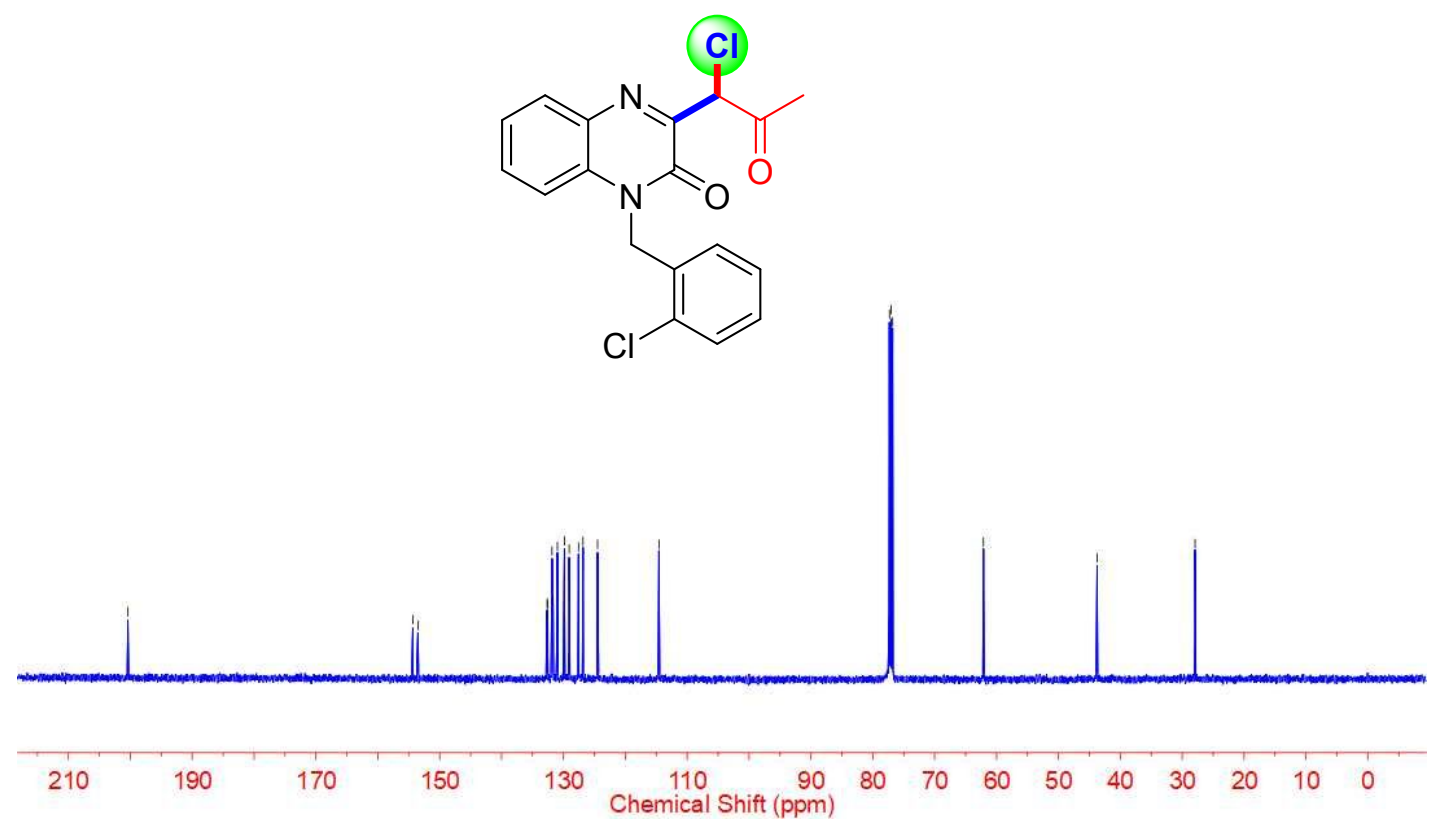

Figure S40. ${ }^{13} \mathrm{C}$ NMR $\left(126 \mathrm{MHz}, \mathrm{CDCl}_{3}\right)$ spectrum of $\mathbf{3 s}$. 


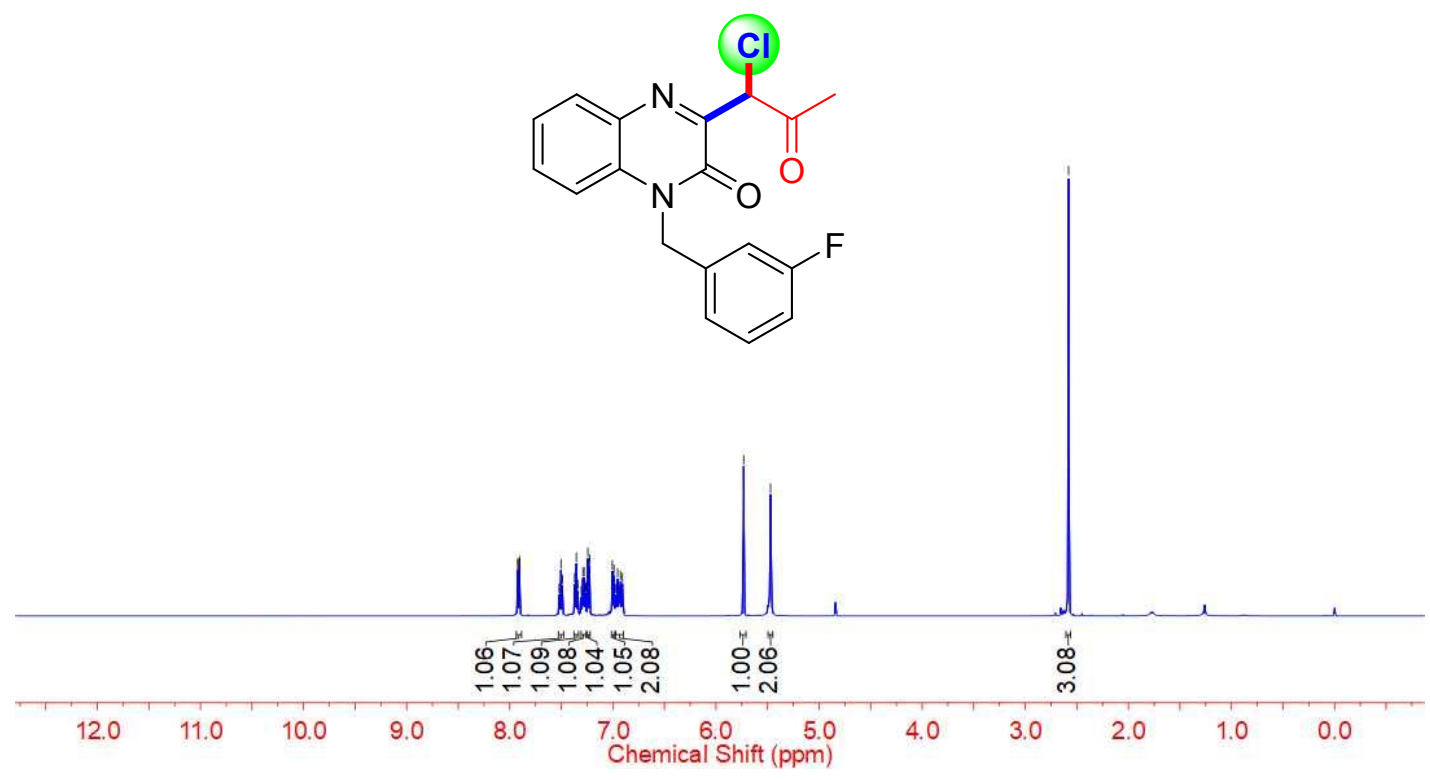

Figure S41. ${ }^{1} \mathrm{H}$ NMR $\left(500 \mathrm{MHz}, \mathrm{CDCl}_{3}\right)$ spectrum of $3 t$.

ָָ

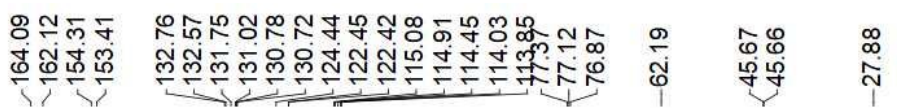<smiles>CC(=O)c1nc2ccccc2n(Cc2cccc(F)c2)c1=O</smiles>

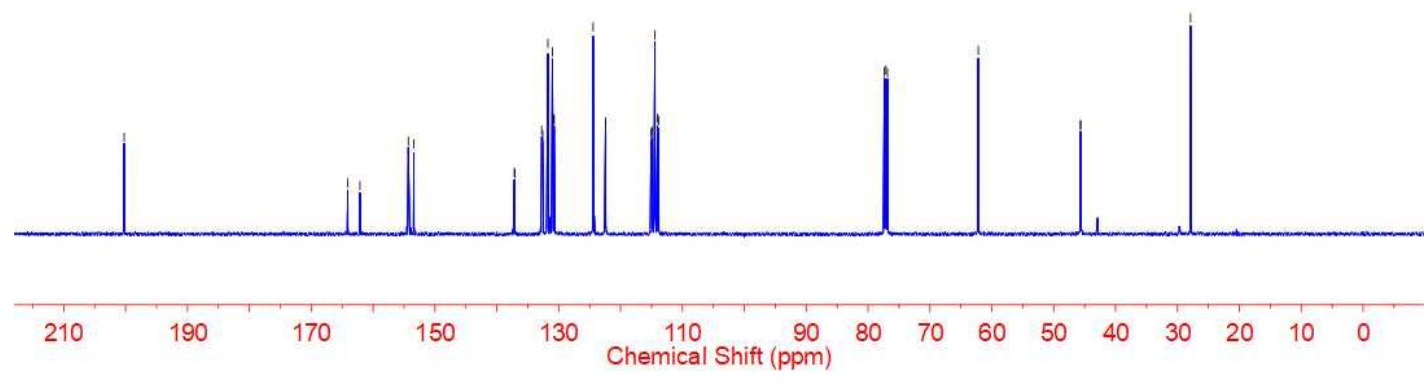

Figure S42. ${ }^{13} \mathrm{C}$ NMR $\left(126 \mathrm{MHz}, \mathrm{CDCl}_{3}\right)$ spectrum of $3 t$. 


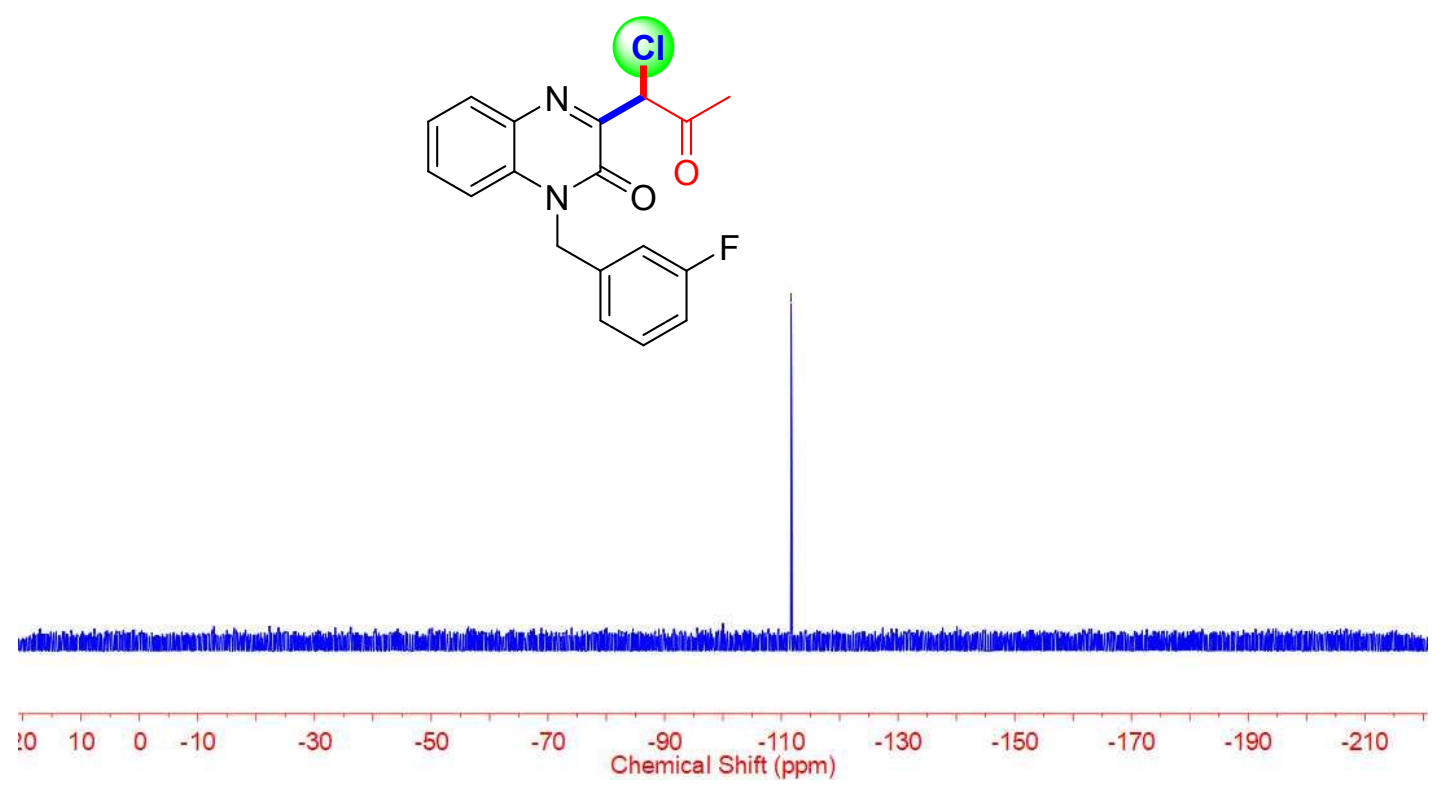

Figure S43. ${ }^{19} \mathrm{~F}$ NMR (471 MHz, $\mathrm{CDCl}_{3}$ ) spectrum of $3 \mathbf{t}$.

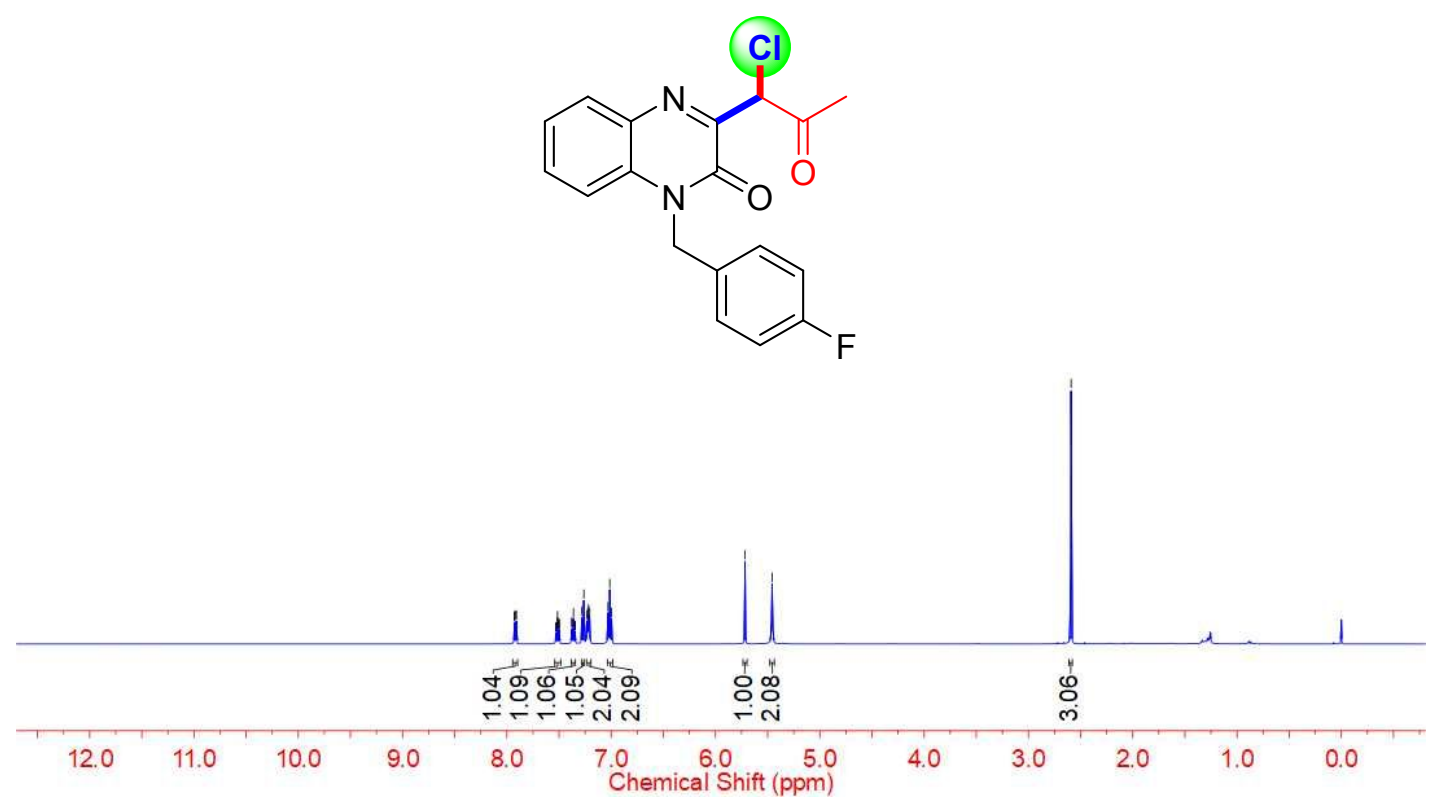

Figure S44. ${ }^{1} \mathrm{H}$ NMR $\left(500 \mathrm{MHz}, \mathrm{CDCl}_{3}\right)$ spectrum of $\mathbf{3 u}$. 
유 암<smiles>CC(=O)C(C)(C)c1nc2ccccc2n(Cc2ccc(F)cc2)c1=O</smiles>

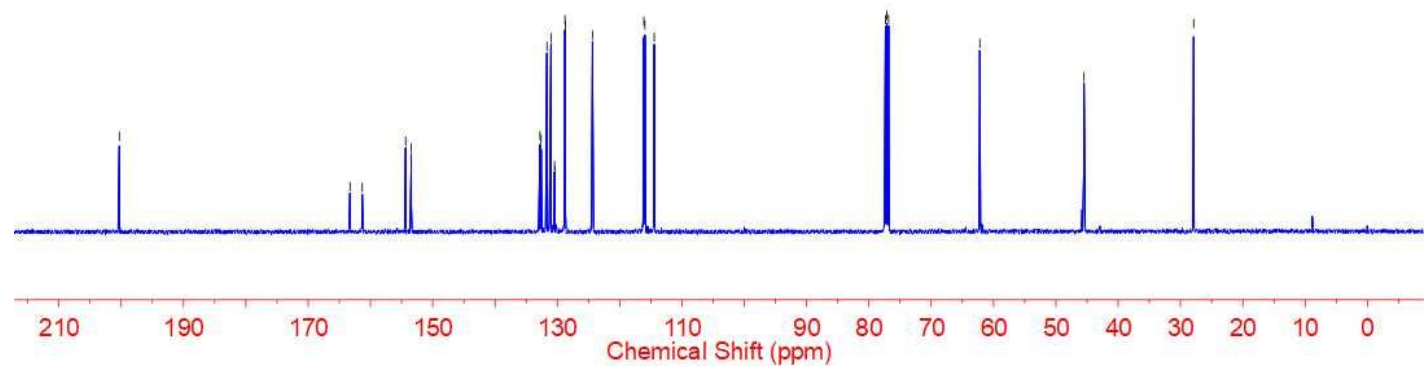

Figure S45. ${ }^{13} \mathrm{C}$ NMR $\left(126 \mathrm{MHz}, \mathrm{CDCl}_{3}\right)$ spectrum of $\mathbf{3 u}$.<smiles>CC(=O)C(C)(C)c1nc2ccccc2n(Cc2ccc(F)cc2)c1=O</smiles>

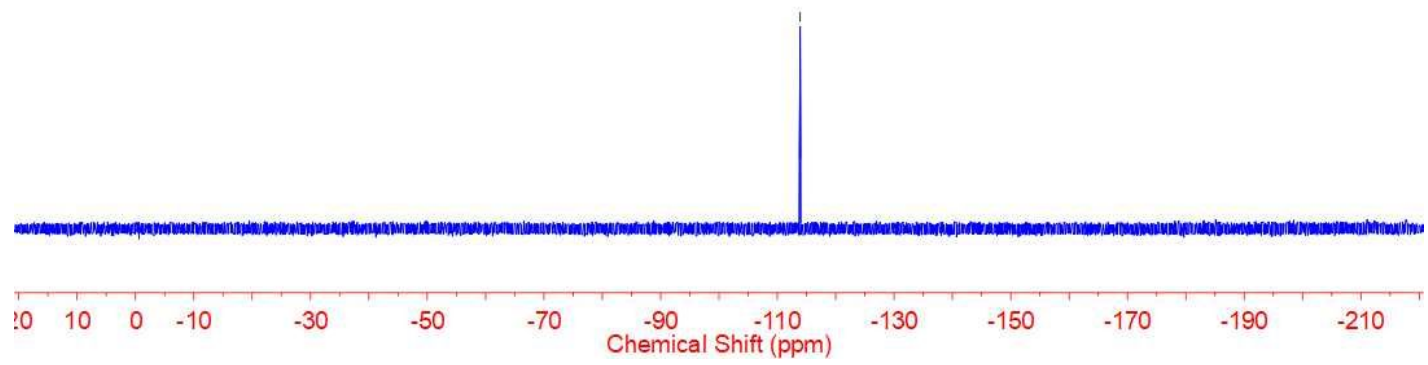

Figure S46. ${ }^{19} \mathrm{~F}$ NMR $\left(471 \mathrm{MHz}, \mathrm{CDCl}_{3}\right)$ spectrum of $\mathbf{3 u}$. 

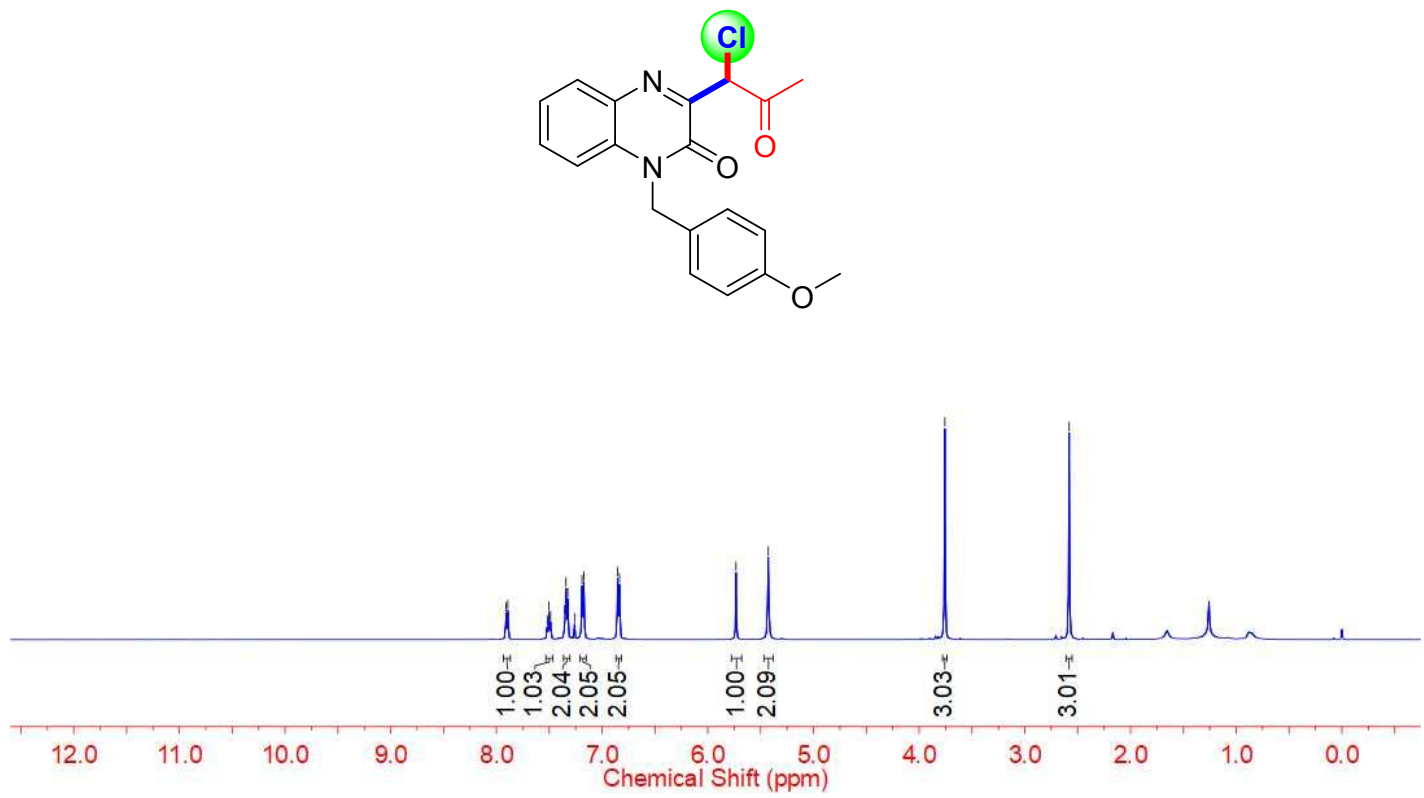

Figure S47. ${ }^{1} \mathrm{H}$ NMR $\left(500 \mathrm{MHz}, \mathrm{CDCl}_{3}\right)$ spectrum of $\mathbf{3 v}$.

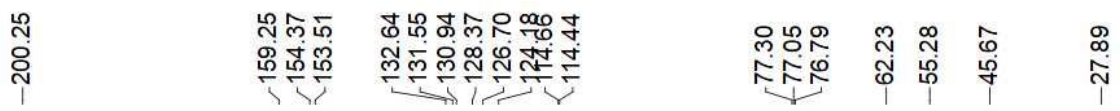

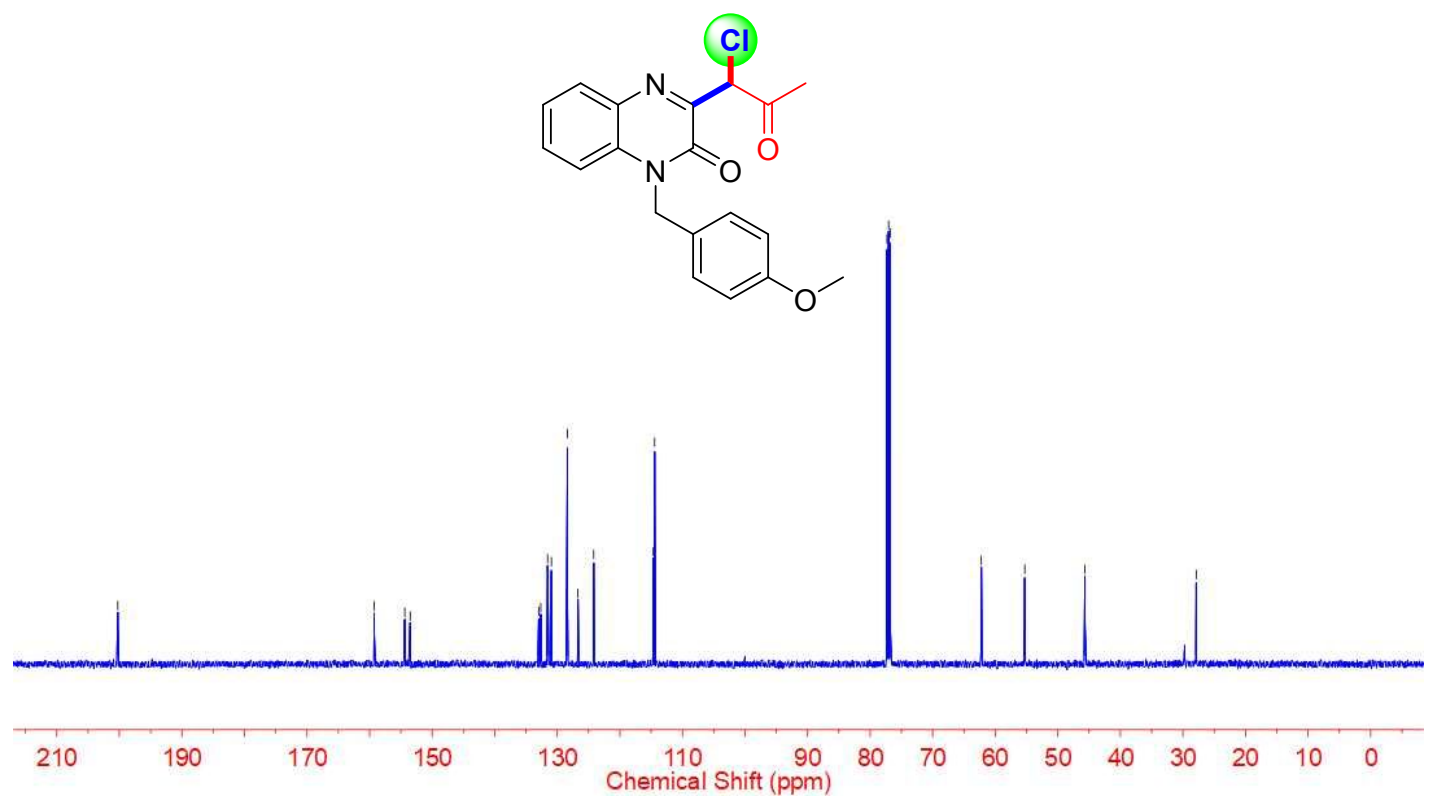

Figure S48. ${ }^{13} \mathrm{C}$ NMR $\left(126 \mathrm{MHz}, \mathrm{CDCl}_{3}\right)$ spectrum of $\mathbf{3 v}$. 


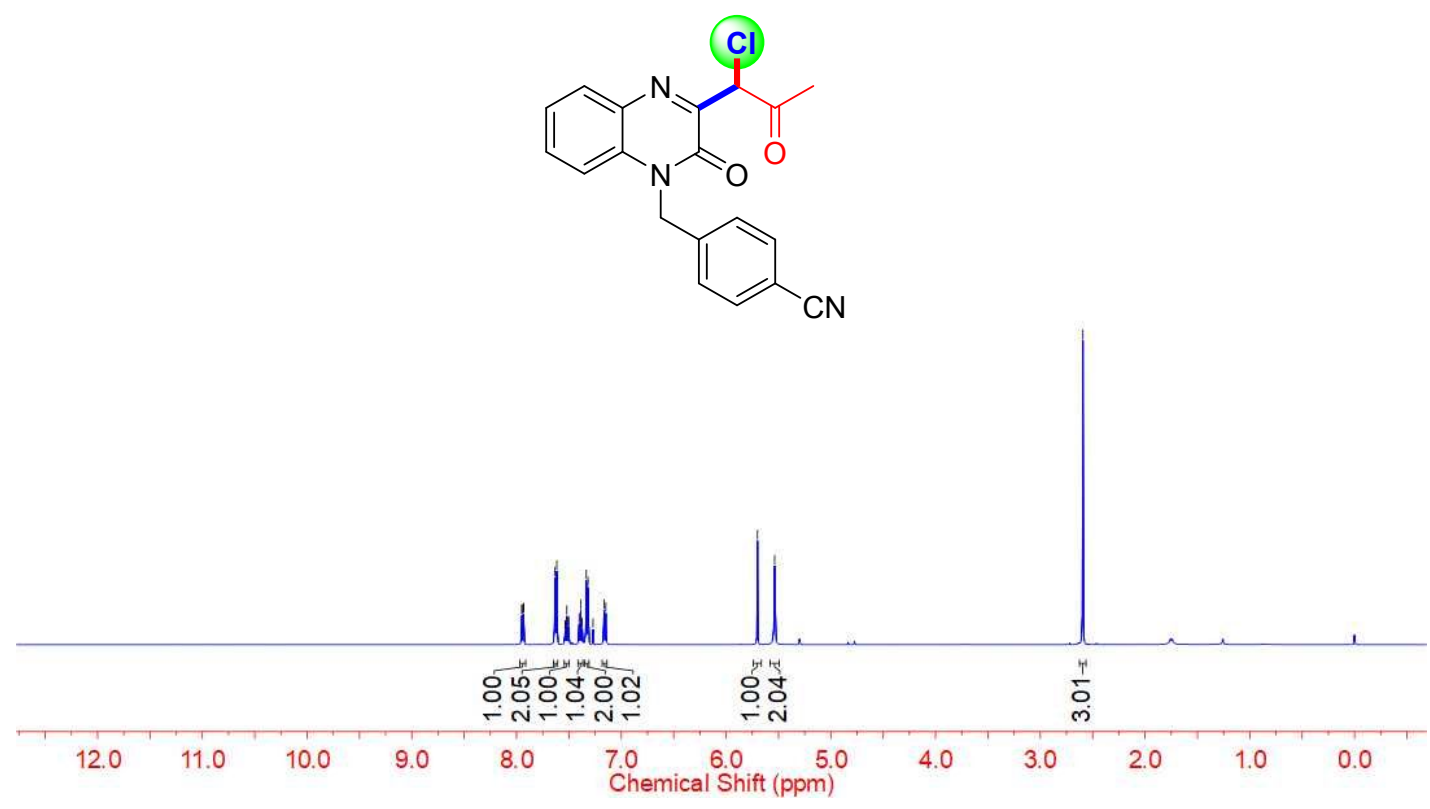

Figure S49. ${ }^{1} \mathrm{H}$ NMR $\left(500 \mathrm{MHz}, \mathrm{CDCl}_{3}\right)$ spectrum of $\mathbf{3 w}$.

ชั่
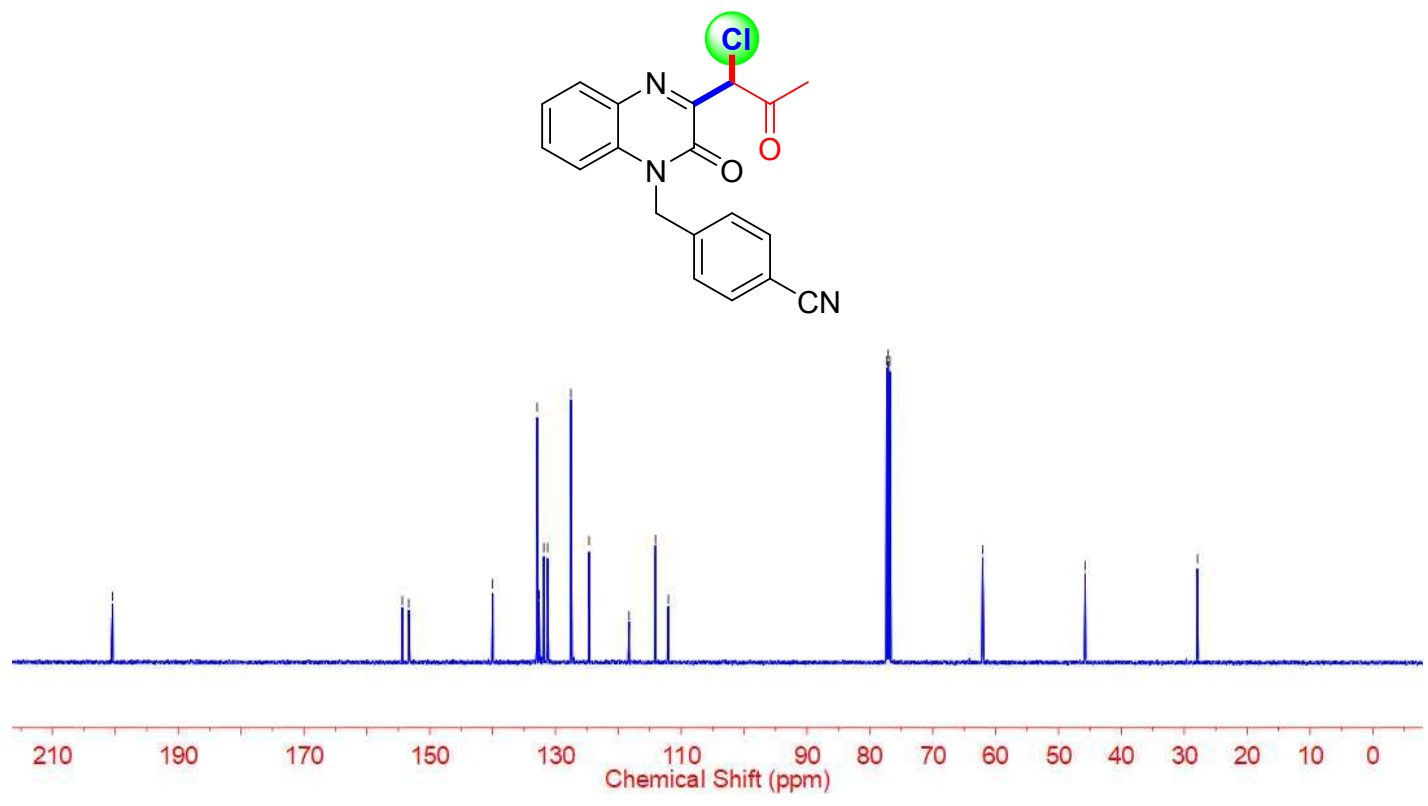

Figure S50. ${ }^{13} \mathrm{C}$ NMR $\left(126 \mathrm{MHz}, \mathrm{CDCl}_{3}\right)$ spectrum of $\mathbf{3 w}$. 


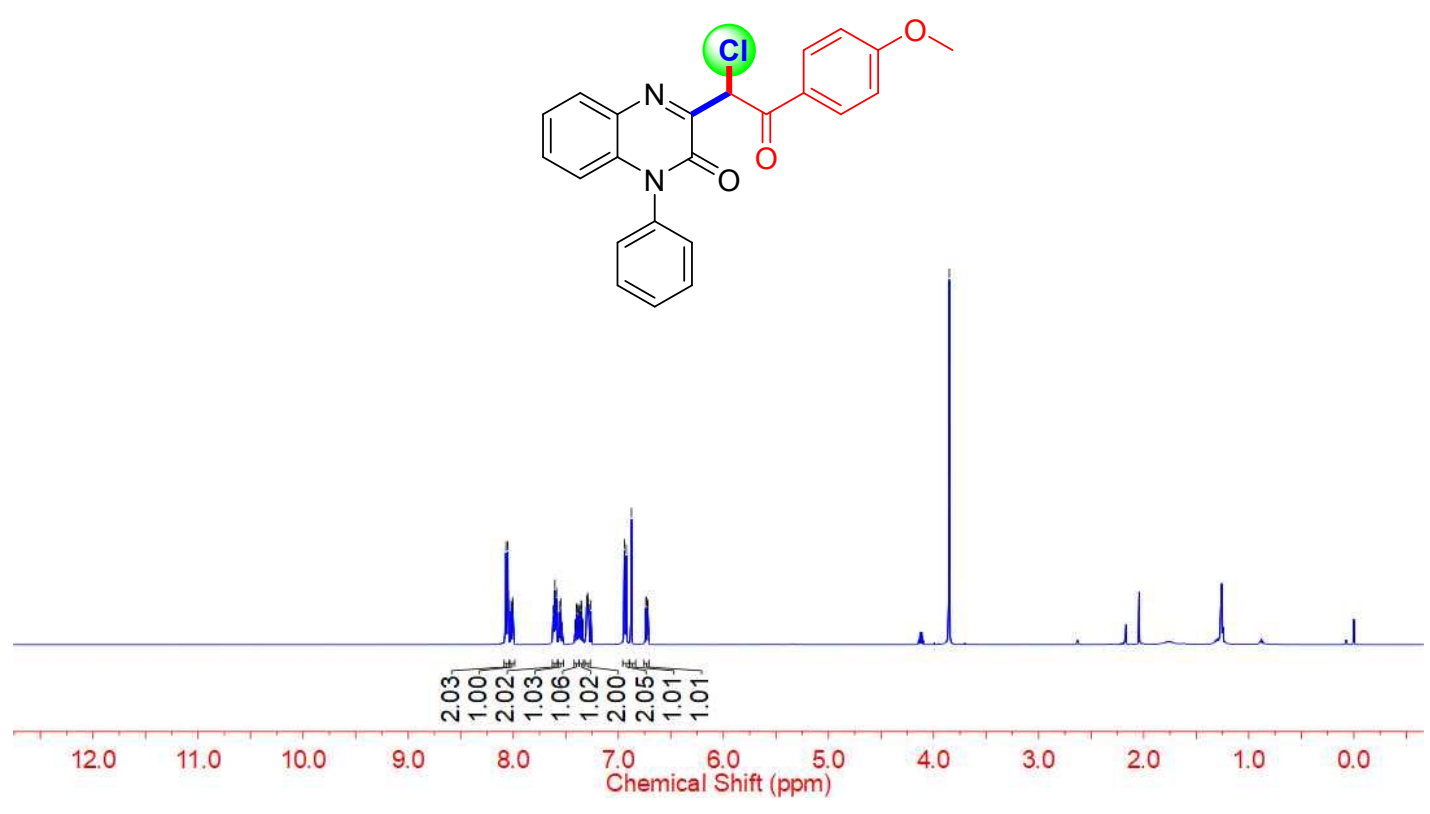

Figure S51. ${ }^{1} \mathrm{H}$ NMR $\left(500 \mathrm{MHz}, \mathrm{CDCl}_{3}\right)$ spectrum of $\mathbf{3 x}$.

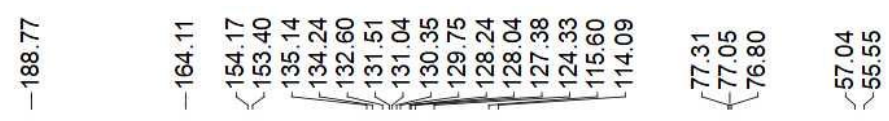<smiles>COc1ccc(C(=O)C(Cl)(Cl)c2nc3ccccc3n(-c3ccccc3)c2=O)cc1</smiles>

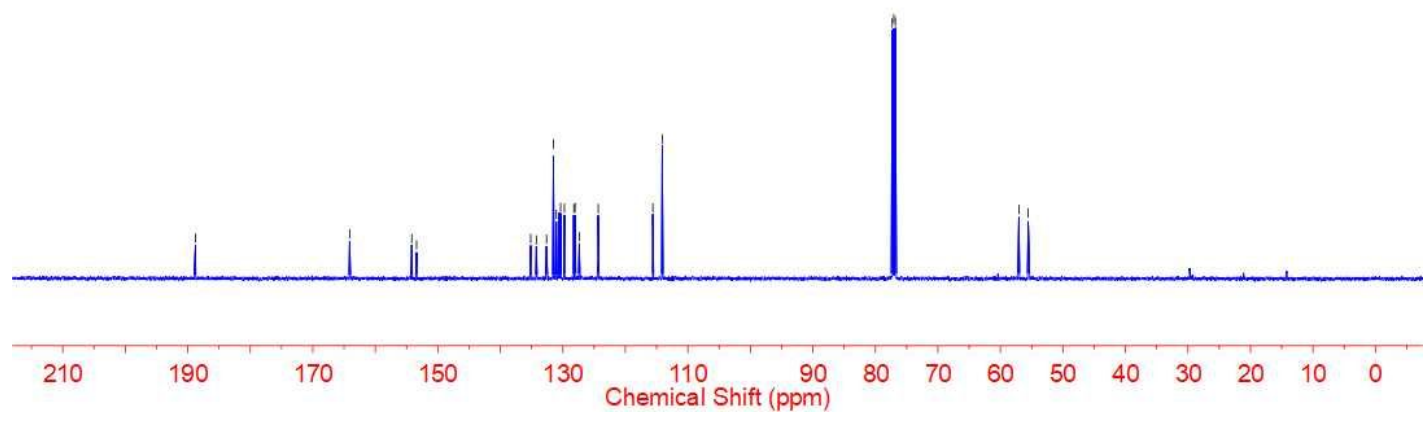

Figure S52. ${ }^{13} \mathrm{C}$ NMR $\left(126 \mathrm{MHz}, \mathrm{CDCl}_{3}\right)$ spectrum of $\mathbf{3 x}$. 

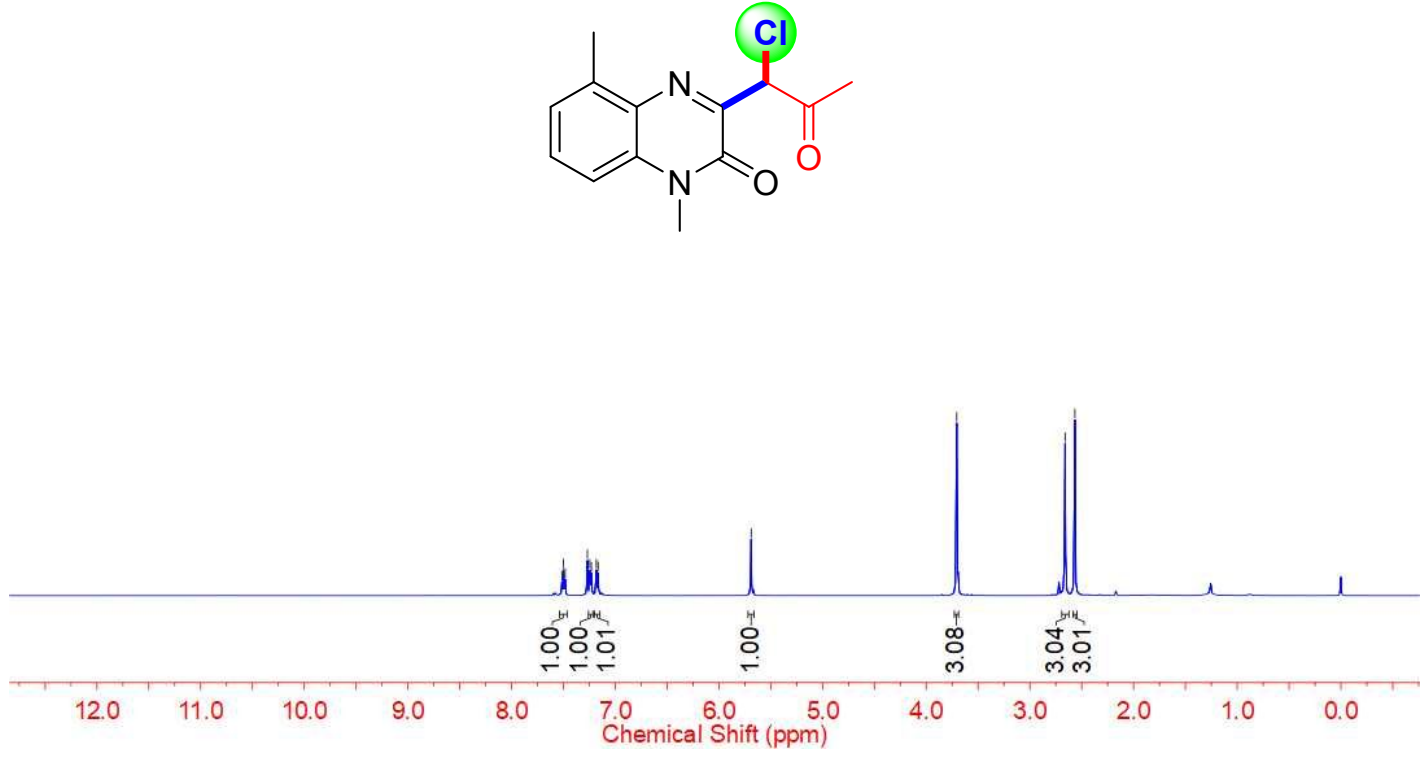

Figure S53. ${ }^{1} \mathrm{H}$ NMR $\left(500 \mathrm{MHz}, \mathrm{CDCl}_{3}\right)$ spectrum of $\mathbf{3 y}$.

i⿱<smiles>CC(=O)C(Cl)(Cl)c1nc2c(C)cccc2n(C)c1=O</smiles>

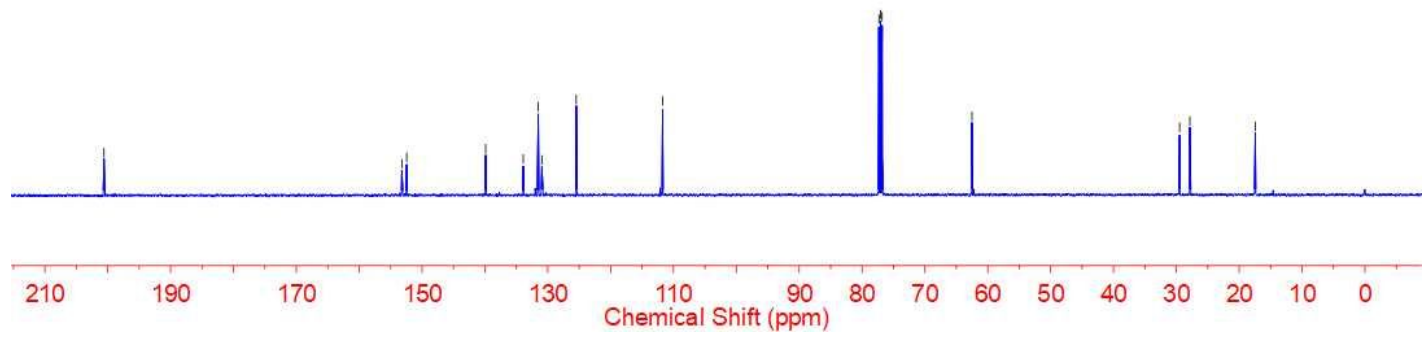

Figure S54. ${ }^{13} \mathrm{C}$ NMR $\left(126 \mathrm{MHz}, \mathrm{CDCl}_{3}\right)$ spectrum of $\mathbf{3 y}$. 
<smiles></smiles>

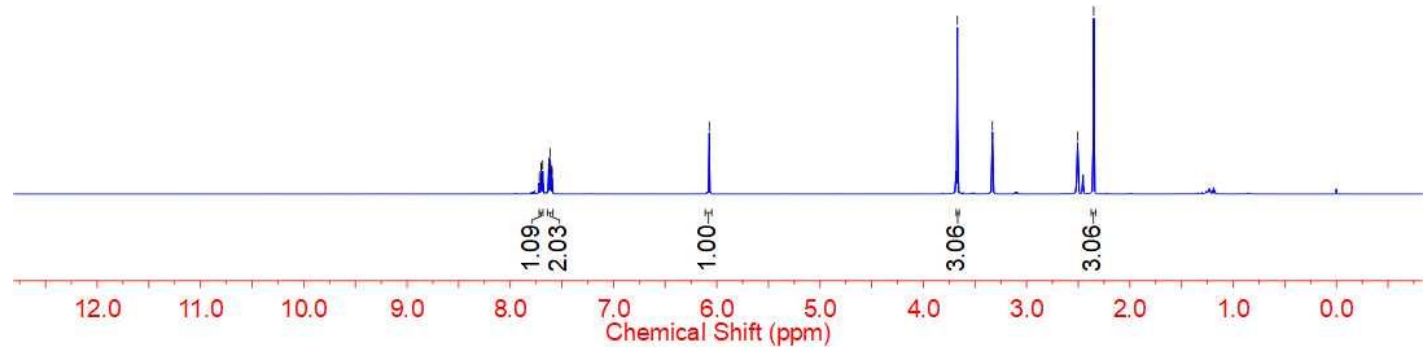

Figure S55. ${ }^{1} \mathrm{H}$ NMR (500 MHz, DMSO) spectrum of $\mathbf{3 z}$.

\begin{tabular}{|c|c|c|c|}
\hline 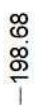 & 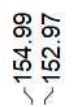 & 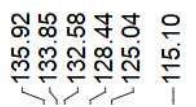 & 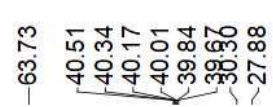 \\
\hline
\end{tabular}<smiles>CC(=O)C(Cl)(Cl)c1nc2c(Cl)cccc2n(C)c1=O</smiles>

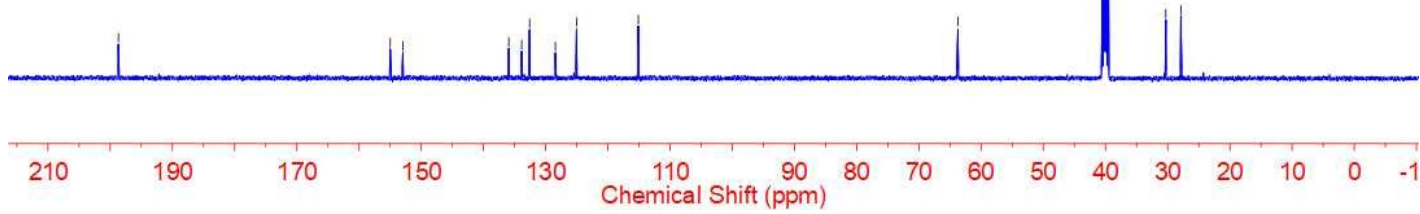

Figure S56. ${ }^{13} \mathrm{C}$ NMR (126 MHz, DMSO) spectrum of 3z. 


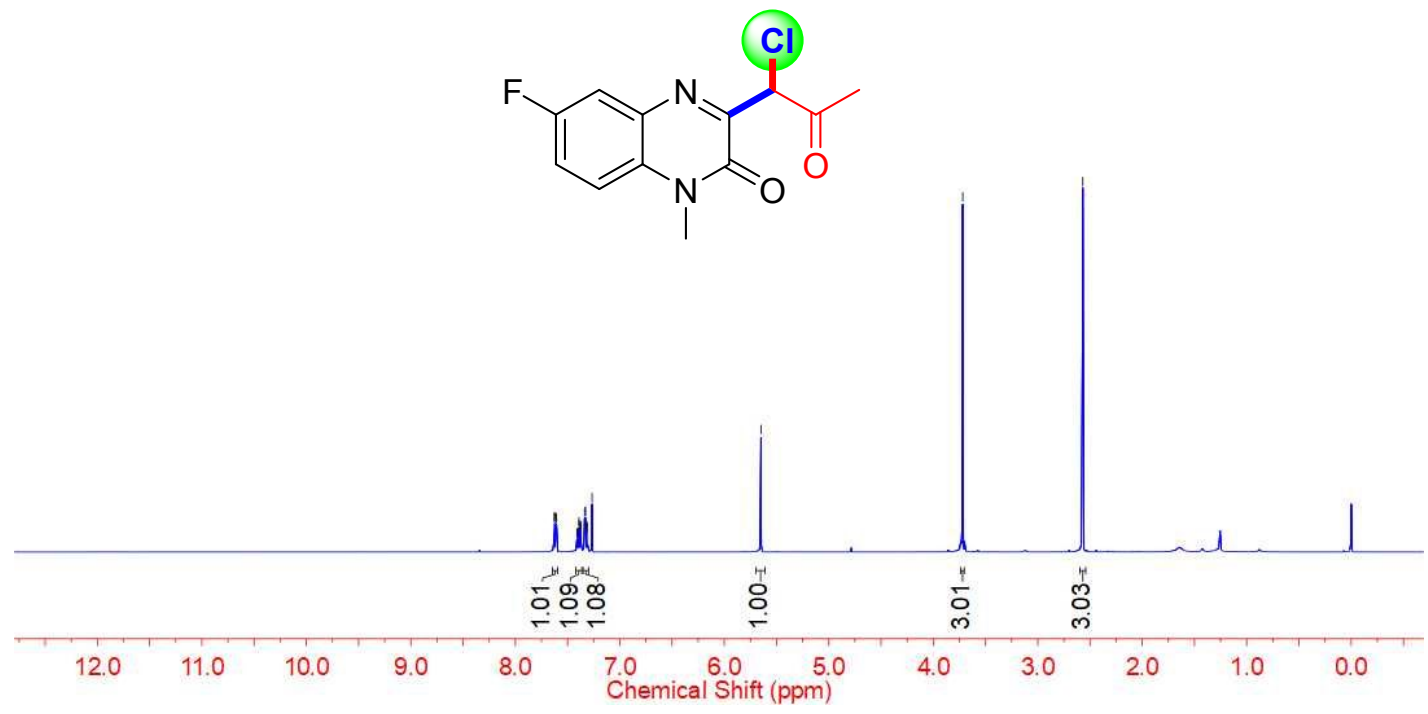

Figure S57. ${ }^{1} \mathrm{H}$ NMR (500 MHz, $\mathrm{CDCl}_{3}$ ) spectrum of $3 \mathbf{a a}$.

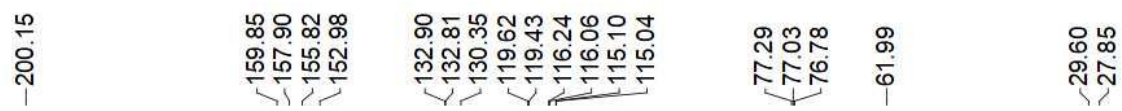<smiles>CC(=O)c1nc2cc(F)ccc2n(C)c1=O</smiles>

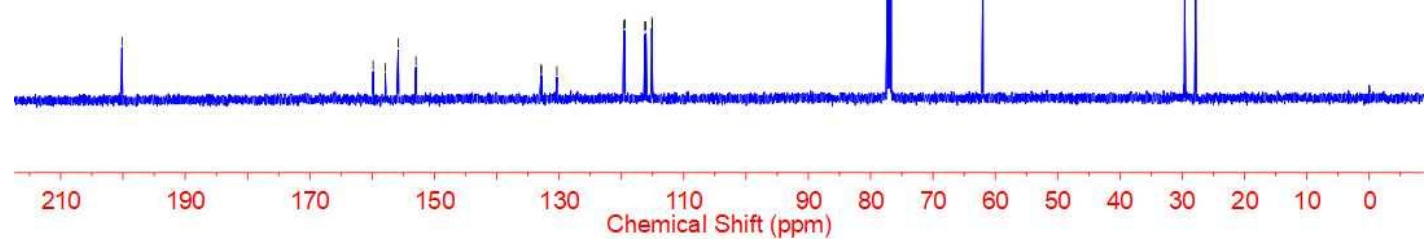

Figure S58. ${ }^{13} \mathrm{C}$ NMR $\left(126 \mathrm{MHz}, \mathrm{CDCl}_{3}\right)$ spectrum of $\mathbf{3 a a}$. 

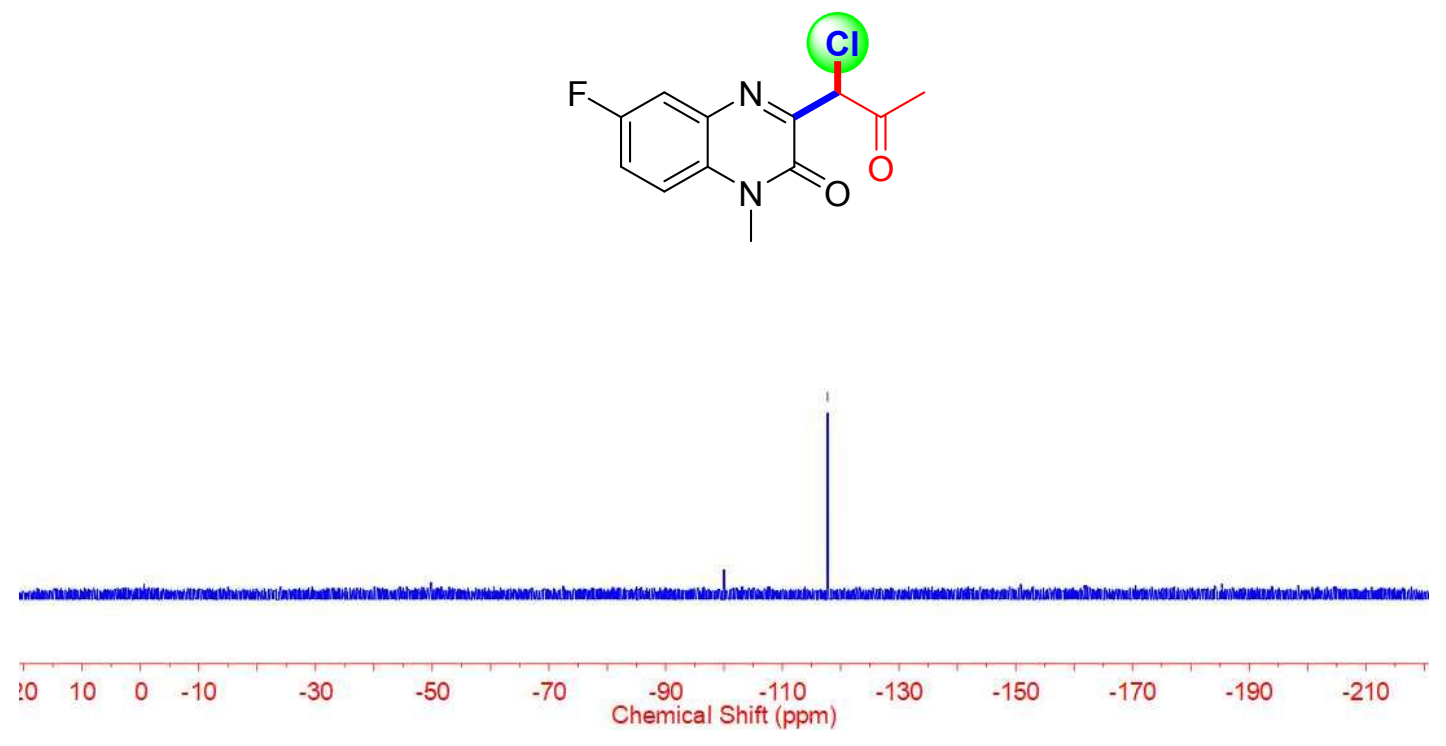

Figure S59. ${ }^{19} \mathrm{~F}$ NMR $\left(471 \mathrm{MHz}, \mathrm{CDCl}_{3}\right)$ spectrum of 3aa.

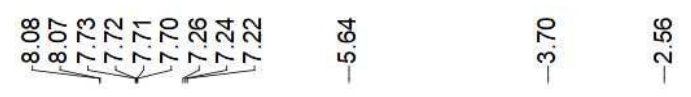<smiles>CC(=O)C(Cl)(Cl)c1nc2cc(Br)ccc2n(C)c1=O</smiles>

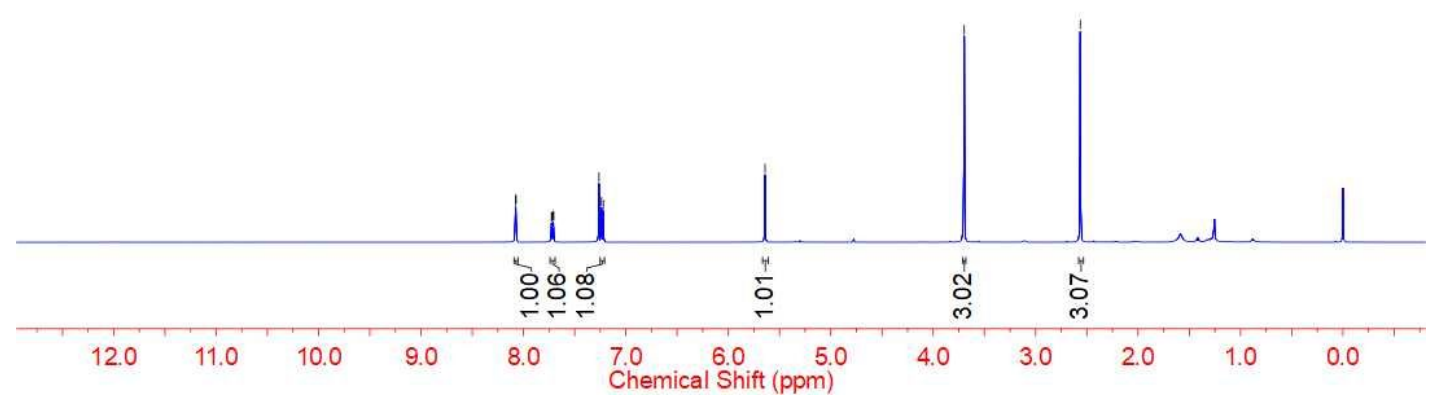

Figure S60. ${ }^{1} \mathrm{H}$ NMR $\left(500 \mathrm{MHz}, \mathrm{CDCl}_{3}\right)$ spectrum of $\mathbf{3 a b}$. 
<smiles>CC(=O)c1nc2cc(Br)ccc2n(C)c1=O</smiles>

Figure S61. ${ }^{13} \mathrm{C}$ NMR (126 MHz, $\left.\mathrm{CDCl}_{3}\right)$ spectrum of 3ab.

\begin{tabular}{|c|}
\hline 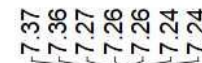 \\
\hline
\end{tabular}<smiles>COc1ccc2nc(C(C)(Cl)C(C)=O)c(=O)n(C)c2c1</smiles>

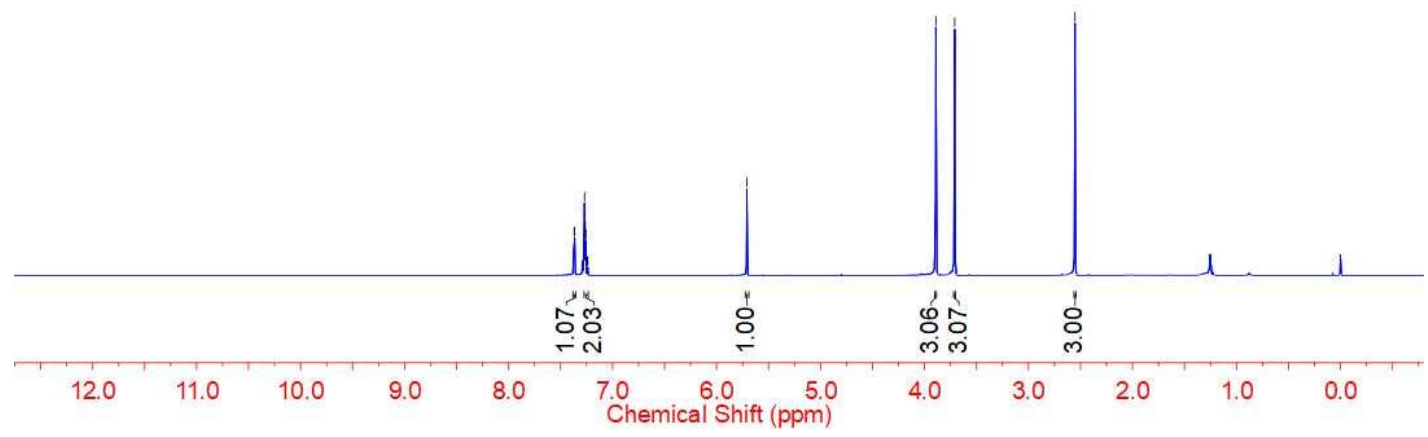

Figure S62. ${ }^{1} \mathrm{H}$ NMR $\left(500 \mathrm{MHz}, \mathrm{CDCl}_{3}\right)$ spectrum of 3ac. 


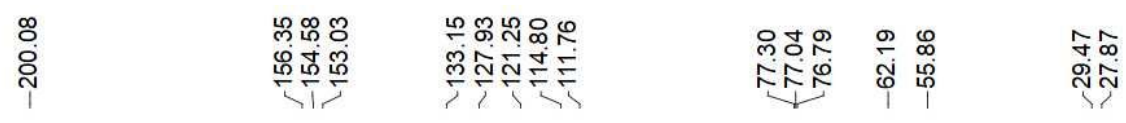<smiles>COc1ccc2nc(C(Cl)(Cl)C(C)=O)c(=O)n(C)c2c1</smiles>

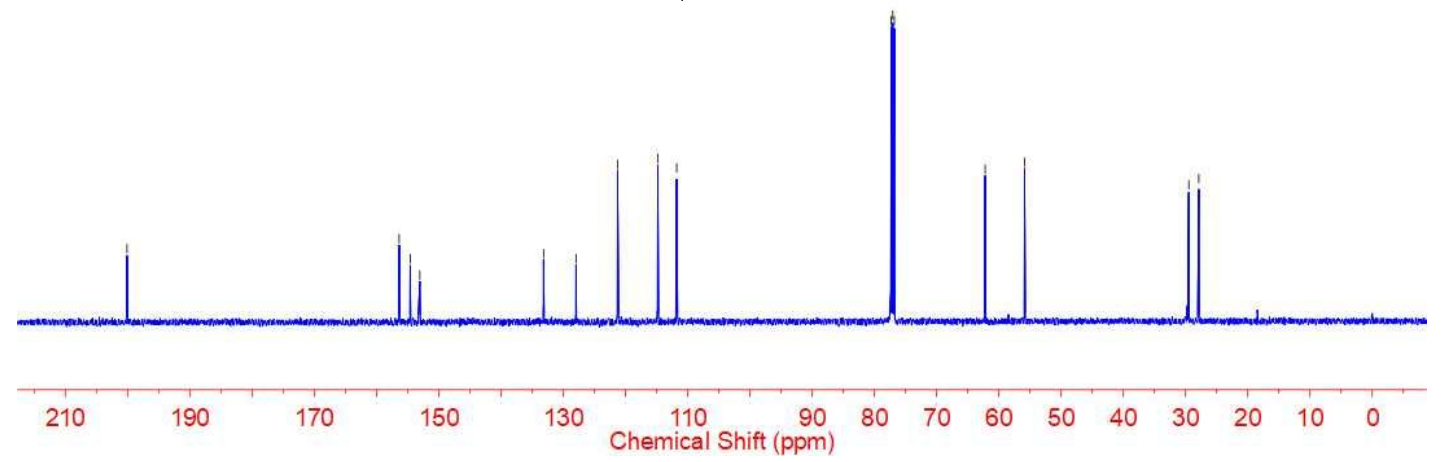

Figure S63. ${ }^{13} \mathrm{C}$ NMR (126 $\left.\mathrm{MHz}, \mathrm{CDCl}_{3}\right)$ spectrum of 3ac.

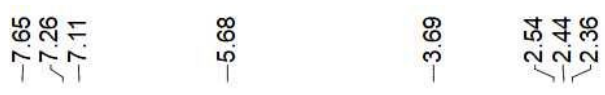<smiles>CC(=O)C(Cl)c1nc2cc(C)c(C)cc2n(C)c1=O</smiles>

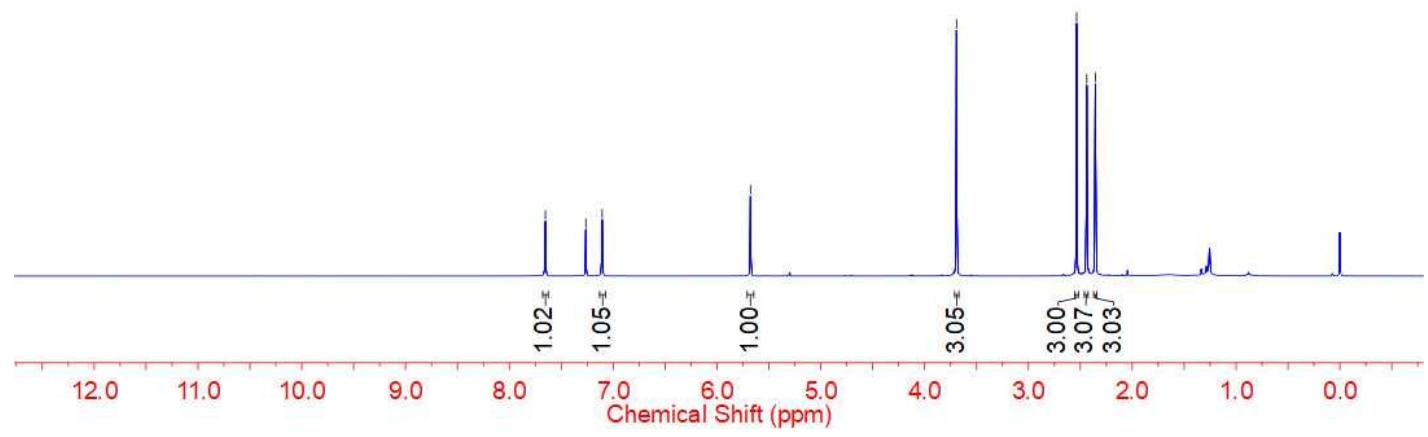

Figure S64. ${ }^{1} \mathrm{H}$ NMR $\left(500 \mathrm{MHz}, \mathrm{CDCl}_{3}\right)$ spectrum of $\mathbf{3 a d}$. 


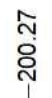<smiles></smiles>

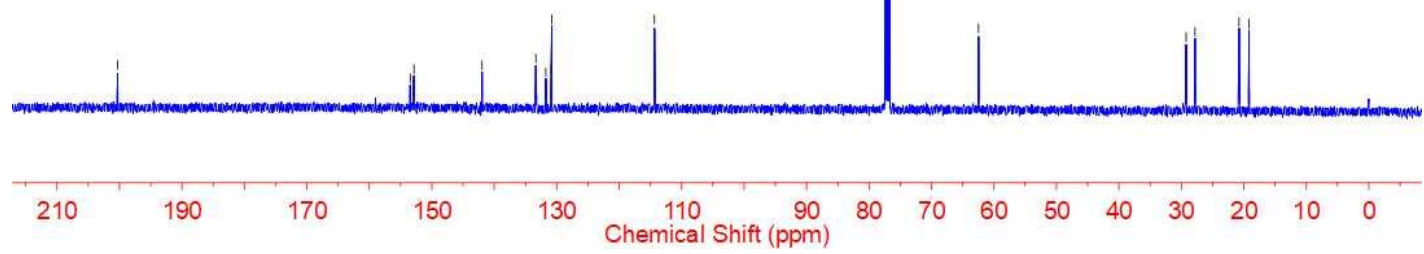

Figure S65. ${ }^{13} \mathrm{C}$ NMR (126 MHz, $\left.\mathrm{CDCl}_{3}\right)$ spectrum of 3ad.<smiles>CC(C)Cc1ccc(C(=O)C(Cl)(Cl)c2nc3ccccc3n(C)c2=O)cc1</smiles>

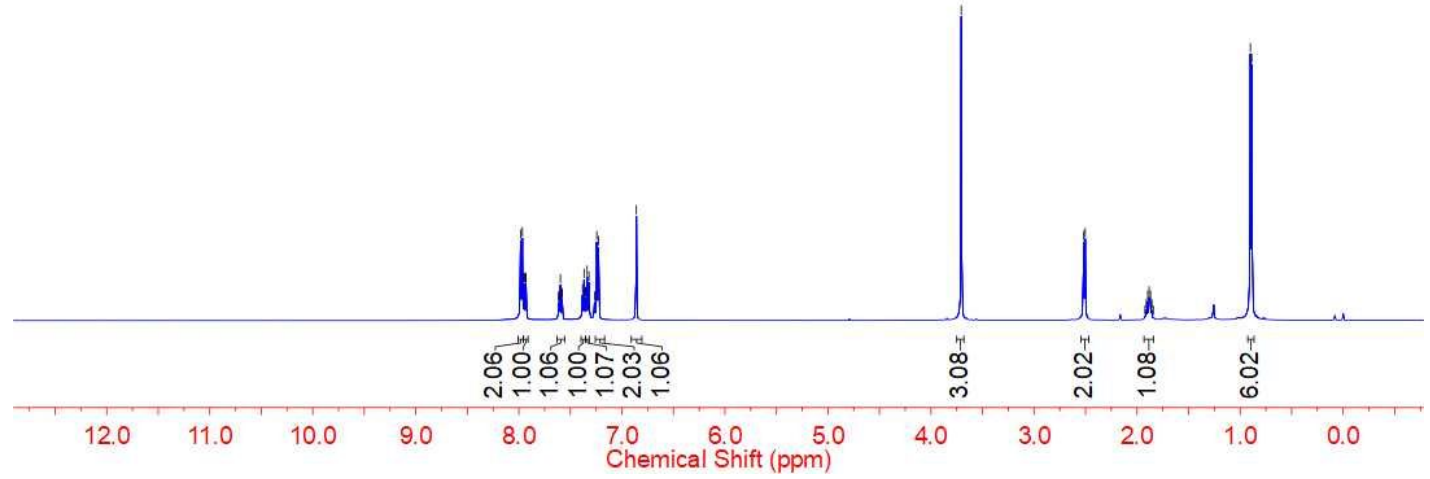

Figure S66. ${ }^{1} \mathrm{H}$ NMR (500 MHz, $\left.\mathrm{CDCl}_{3}\right)$ spectrum of 3ae. 


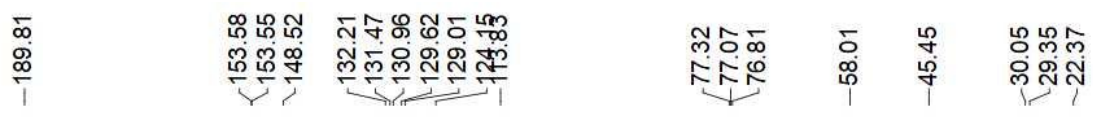
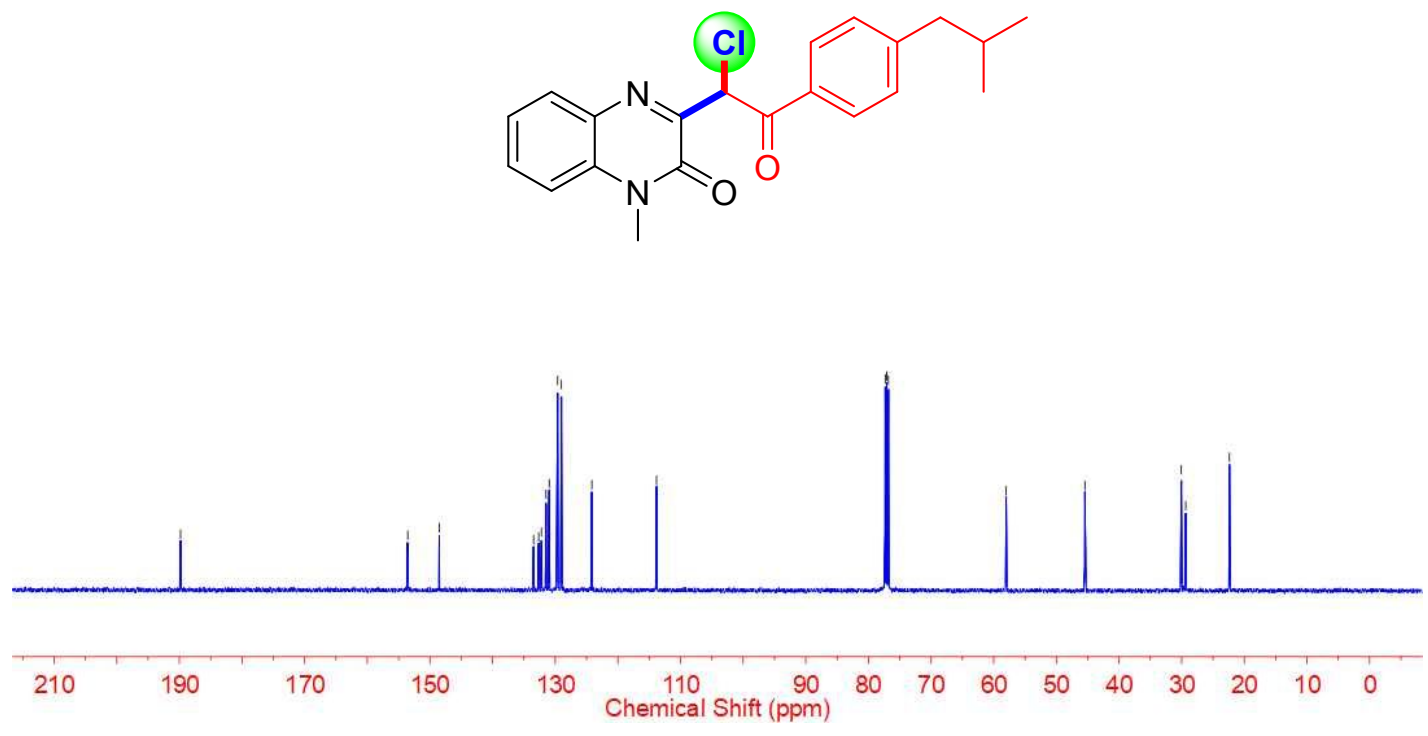

Figure S67. ${ }^{13} \mathrm{C}$ NMR $\left(126 \mathrm{MHz}, \mathrm{CDCl}_{3}\right)$ spectrum of 3ae.

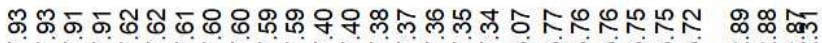

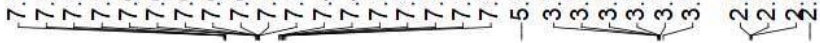

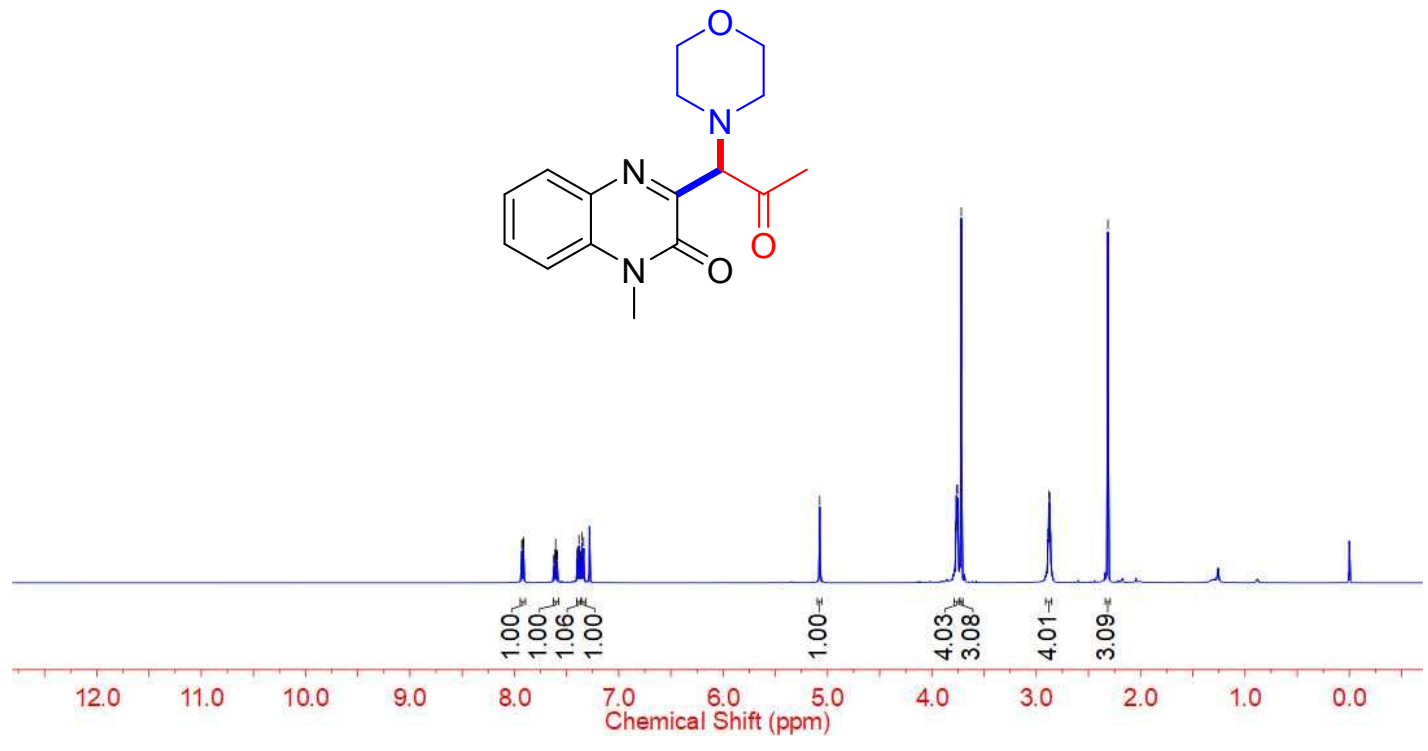

Figure S68. ${ }^{1} \mathrm{H}$ NMR $\left(500 \mathrm{MHz}, \mathrm{CDCl}_{3}\right)$ spectrum of $4 \mathbf{a}$. 


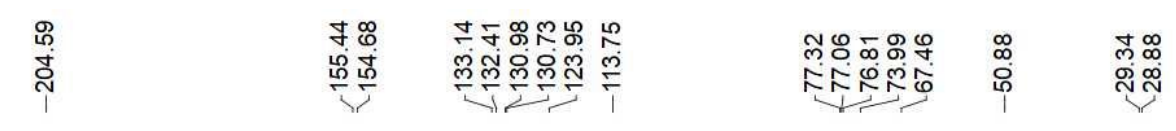<smiles>CC(=O)[C@H](c1nc2ccccc2n(C)c1=O)N1CCOCC1</smiles>

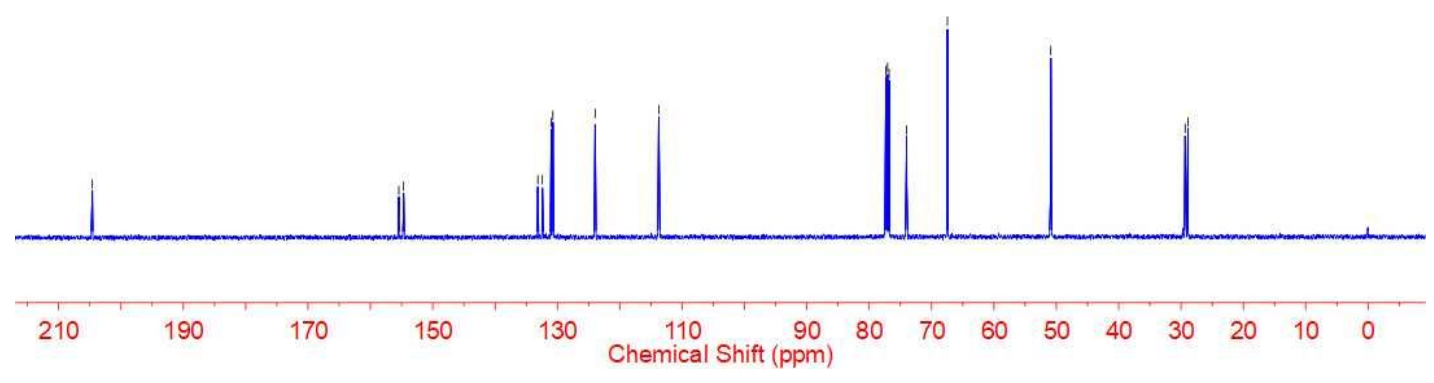

Figure S69. ${ }^{13} \mathrm{C}$ NMR $\left(126 \mathrm{MHz}, \mathrm{CDCl}_{3}\right)$ spectrum of $4 \mathbf{a}$.<smiles>CCOP(=O)(OCC)C(C(C)=O)c1nc2ccccc2n(C)c1=O</smiles>

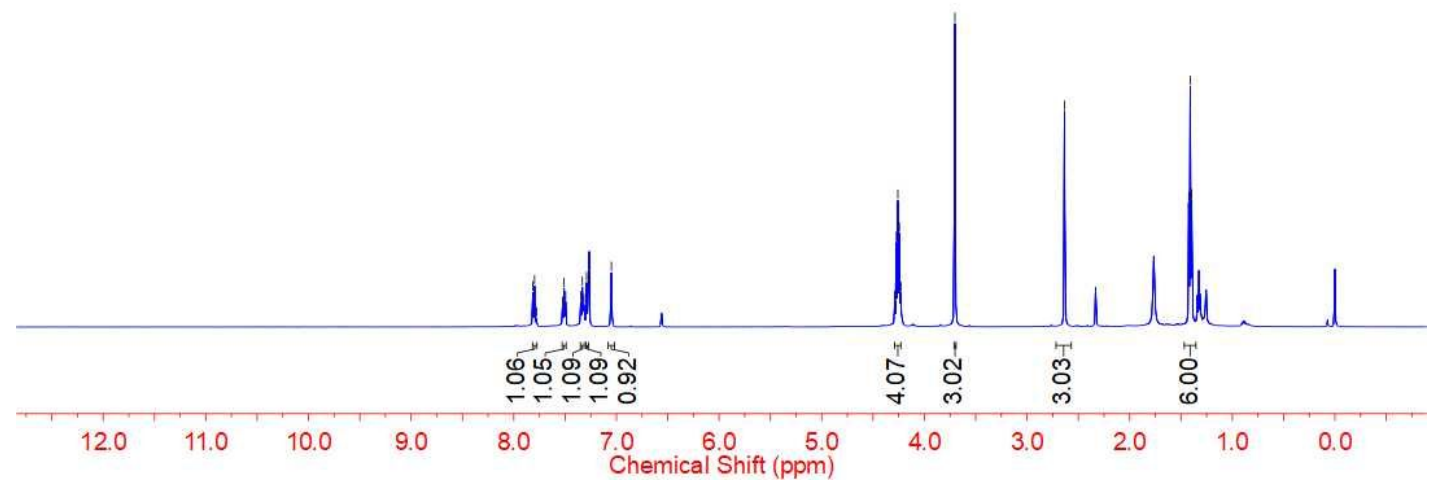

Figure S70. ${ }^{1} \mathrm{H}$ NMR $\left(500 \mathrm{MHz}, \mathrm{CDCl}_{3}\right)$ spectrum of $\mathbf{4 b}$. 

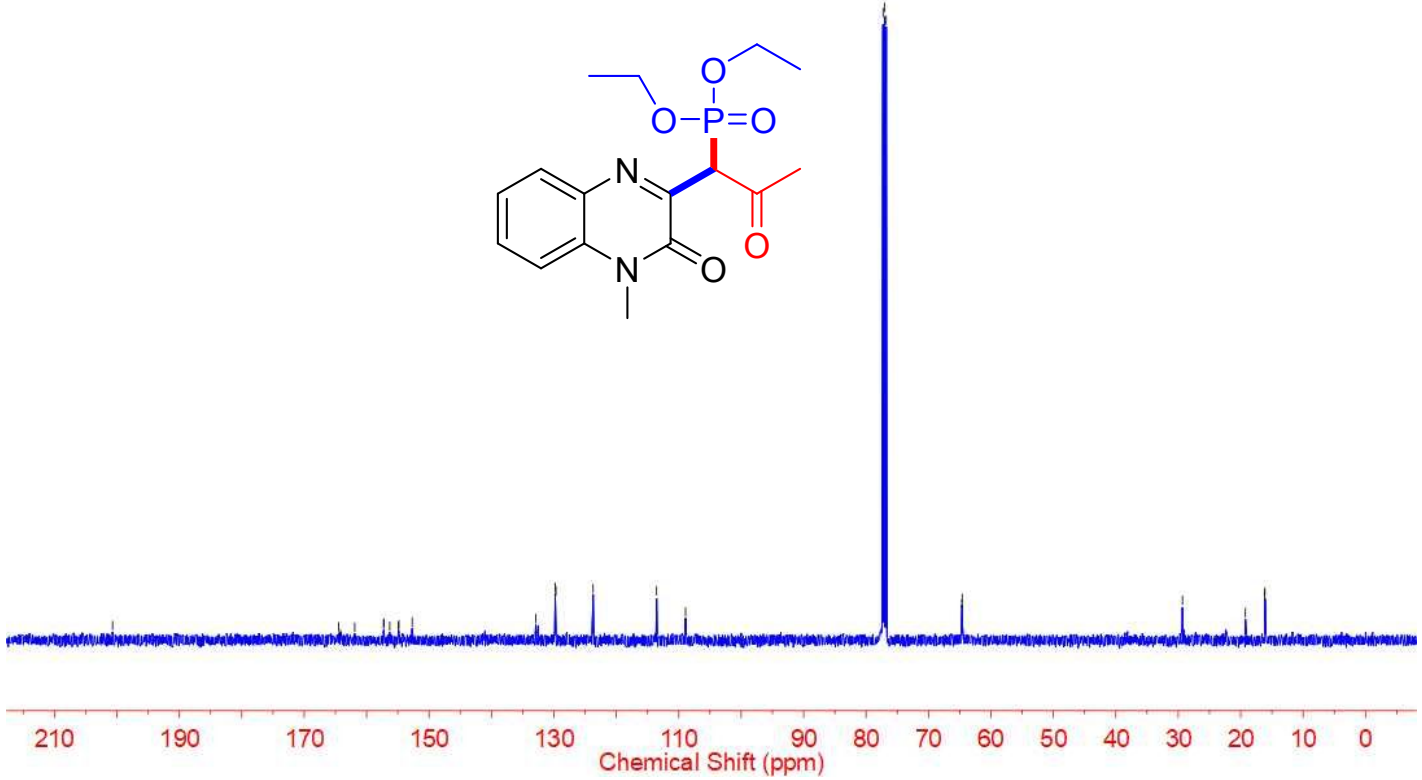

Figure S71. ${ }^{13} \mathrm{C}$ NMR (126 MHz, $\left.\mathrm{CDCl}_{3}\right)$ spectrum of $4 \mathbf{b}$.
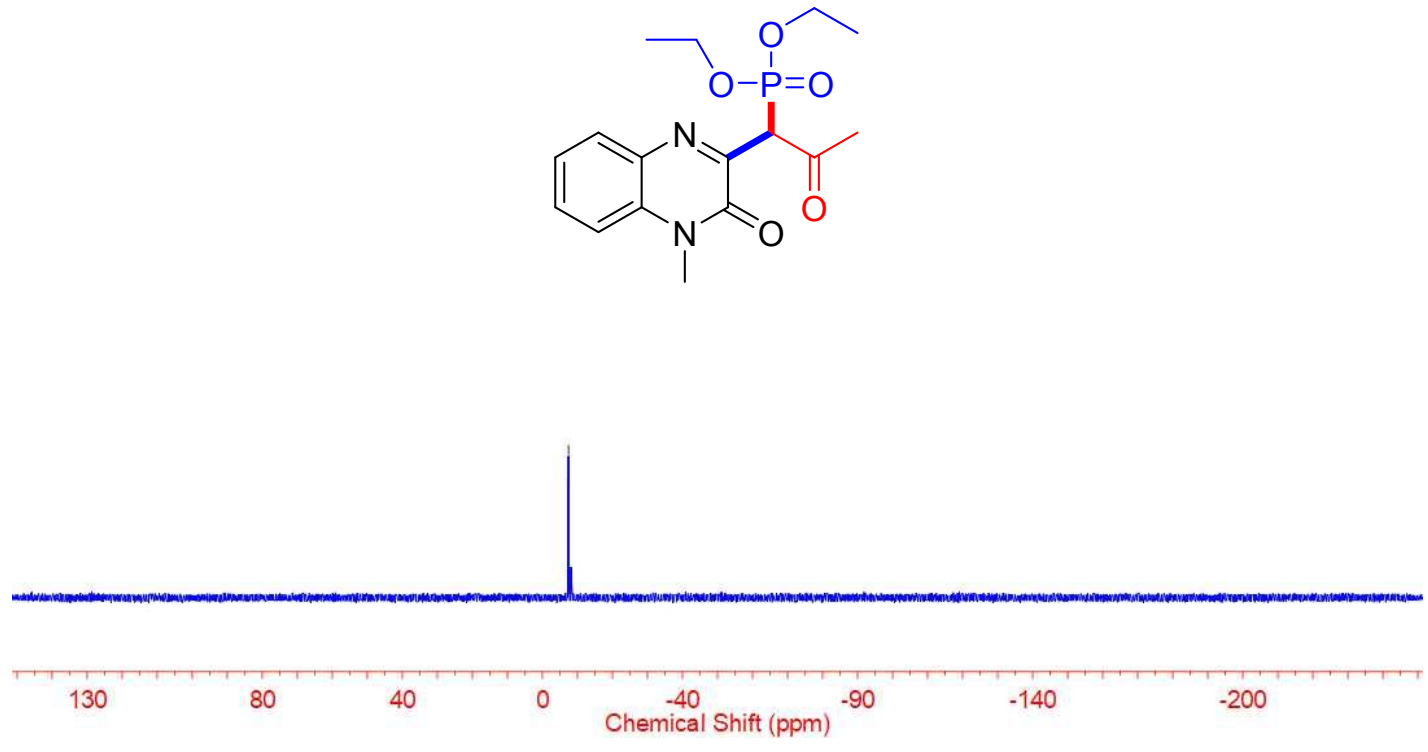

Figure S72. ${ }^{31} \mathrm{P}$ NMR $\left(202 \mathrm{MHz}, \mathrm{CDCl}_{3}\right)$ spectrum of $\mathbf{4 b}$. 
<smiles>Cn1c(=O)c(CCl)nc2ccccc21</smiles>

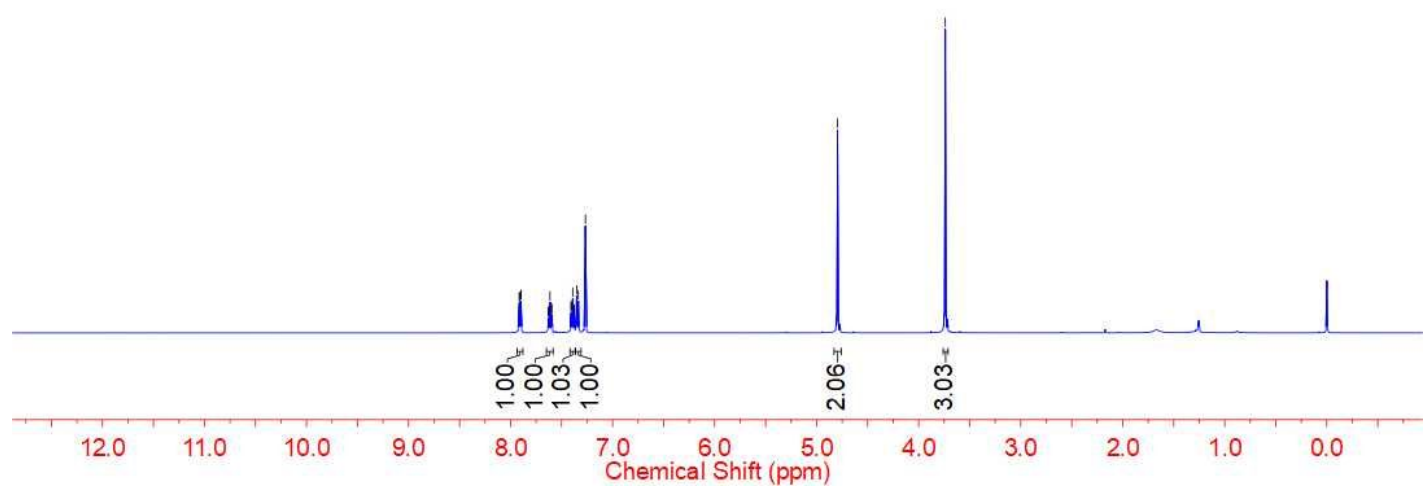

Figure S73. ${ }^{1} \mathrm{H}$ NMR $\left(500 \mathrm{MHz}, \mathrm{CDCl}_{3}\right)$ spectrum of $\mathbf{4 c}$.

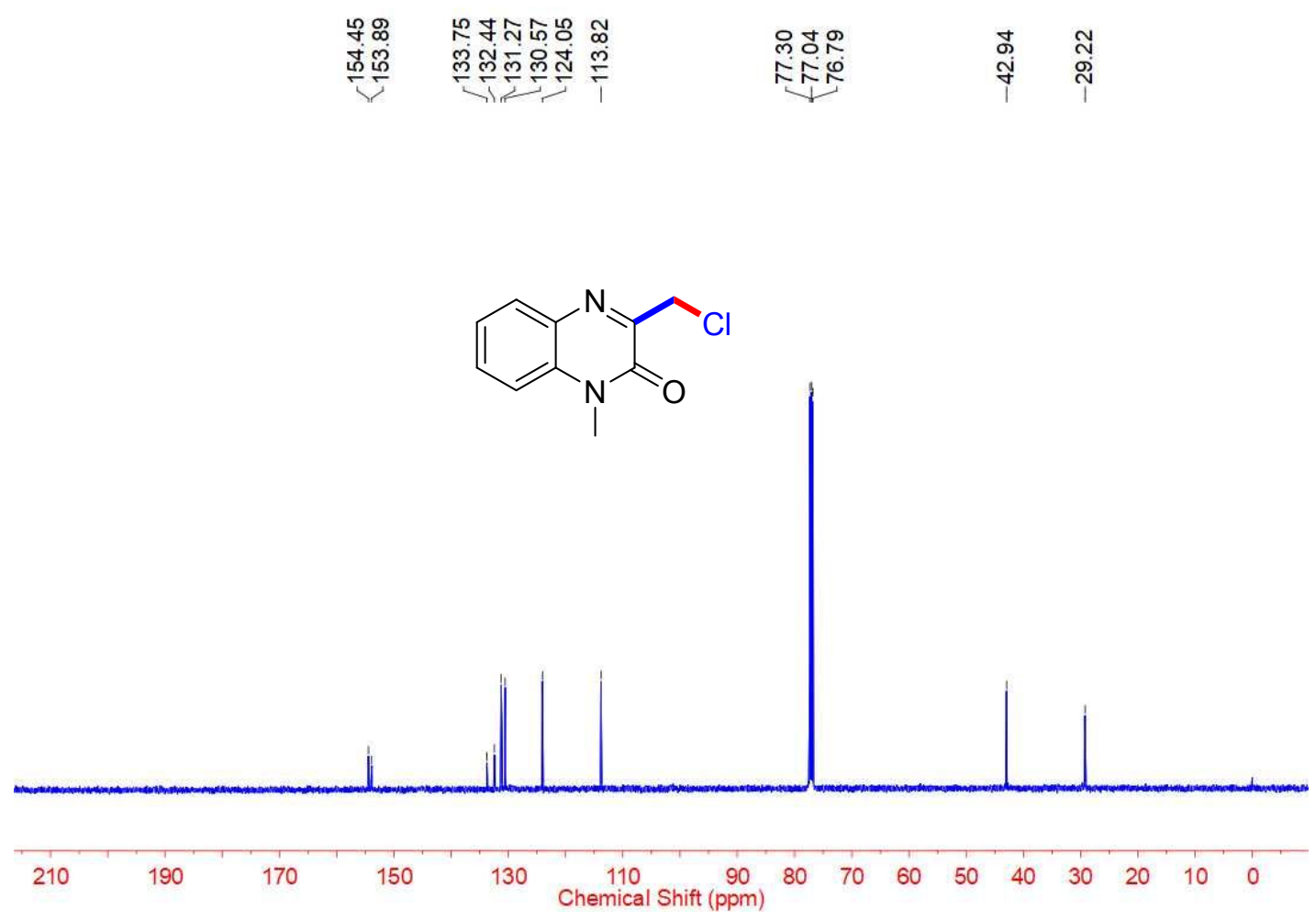

Figure S74. ${ }^{13} \mathrm{C}$ NMR $\left(126 \mathrm{MHz}, \mathrm{CDCl}_{3}\right)$ spectrum of $\mathbf{4 c}$. 
<smiles>Cc1ccc(S(=O)(=O)Cc2nc3ccccc3n(C)c2=O)cc1</smiles>

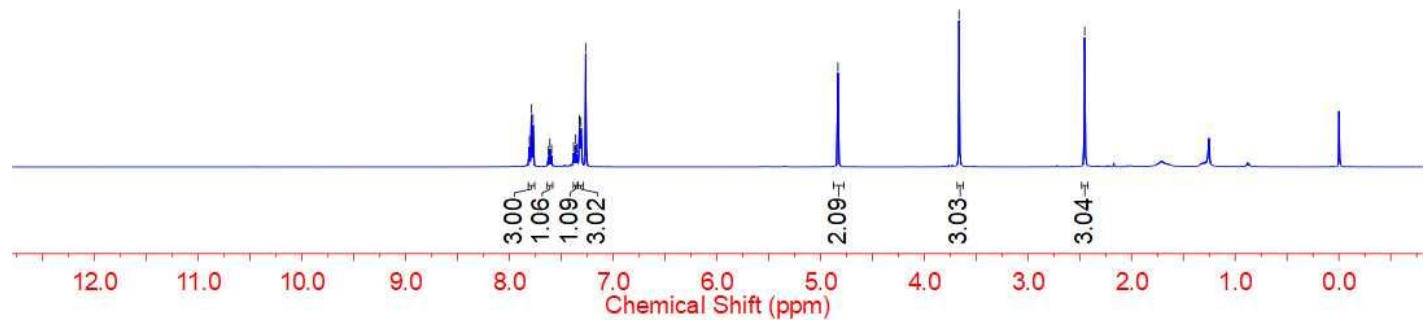

Figure S75. ${ }^{1} \mathrm{H}$ NMR $\left(500 \mathrm{MHz}, \mathrm{CDCl}_{3}\right)$ spectrum of $\mathbf{4 d}$.
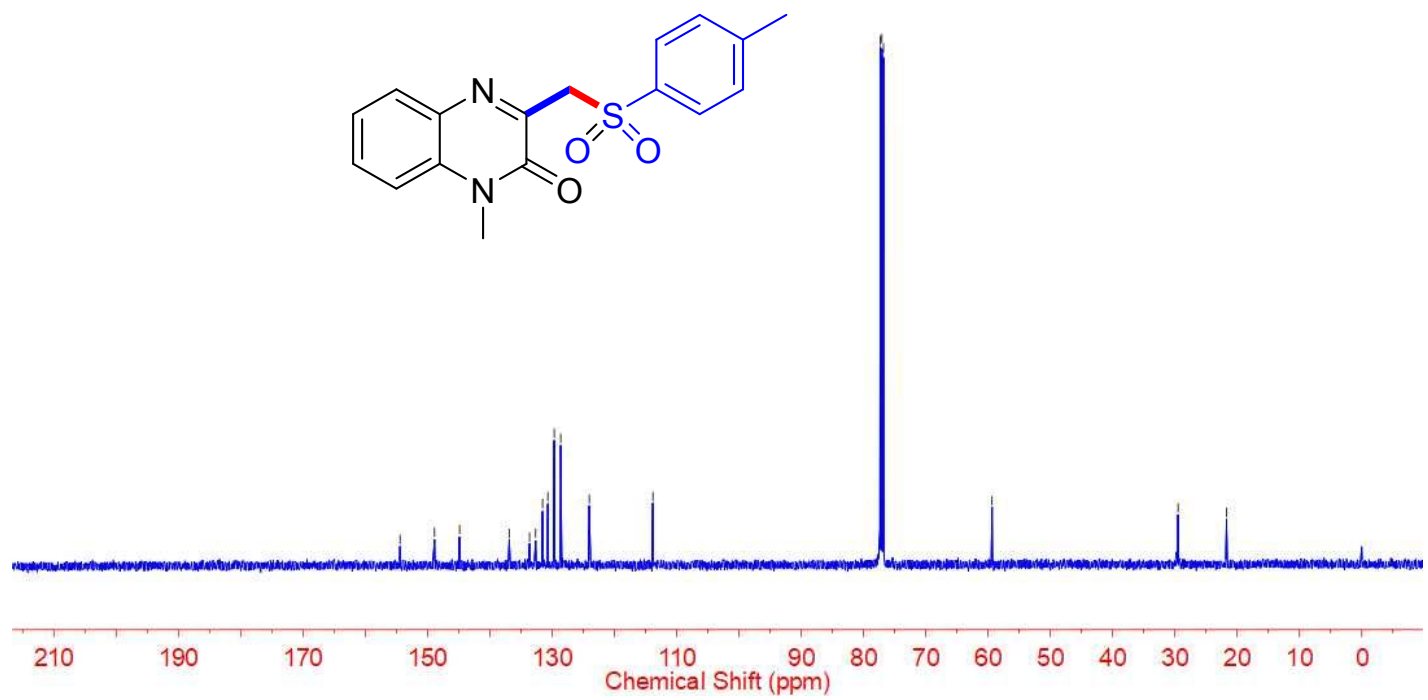

Figure S76. ${ }^{13} \mathrm{C}$ NMR $\left(126 \mathrm{MHz}, \mathrm{CDCl}_{3}\right)$ spectrum of $\mathbf{4 d}$. 


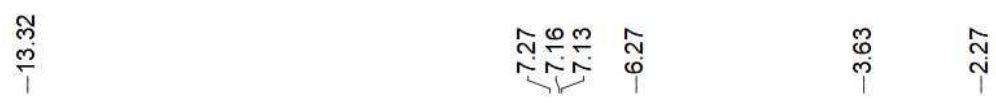<smiles>CC(=O)/C=C1\Nc2ccccc2N(C)C1=O</smiles>

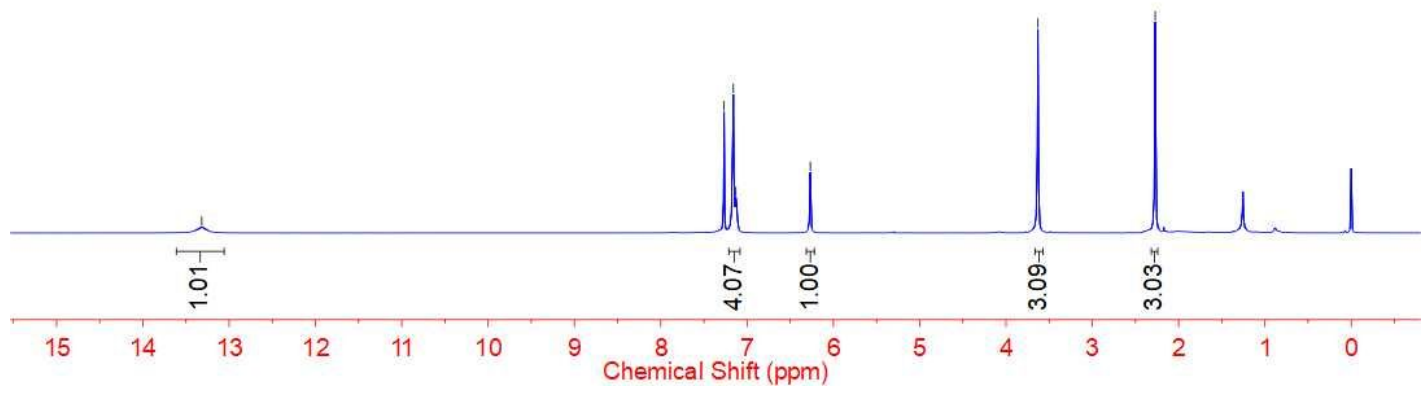

Figure S77. ${ }^{1} \mathrm{H} \mathrm{NMR}\left(500 \mathrm{MHz}, \mathrm{CDCl}_{3}\right)$ spectrum of $\mathbf{5}$.<smiles>CC(=O)C[C@@H]1Nc2ccccc2N(C)C1=O</smiles>

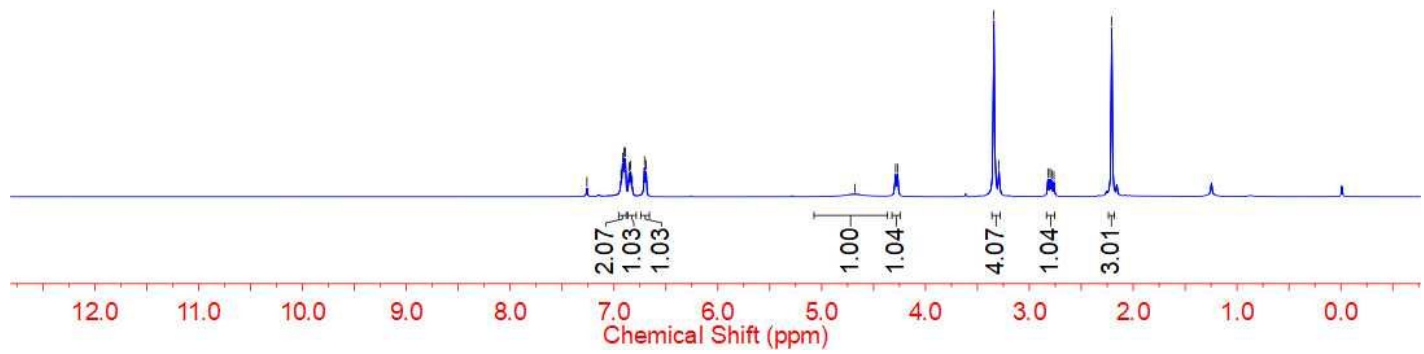

Figure S78. ${ }^{1} \mathrm{H}$ NMR $\left(500 \mathrm{MHz}, \mathrm{CDCl}_{3}\right)$ spectrum of 6 . 

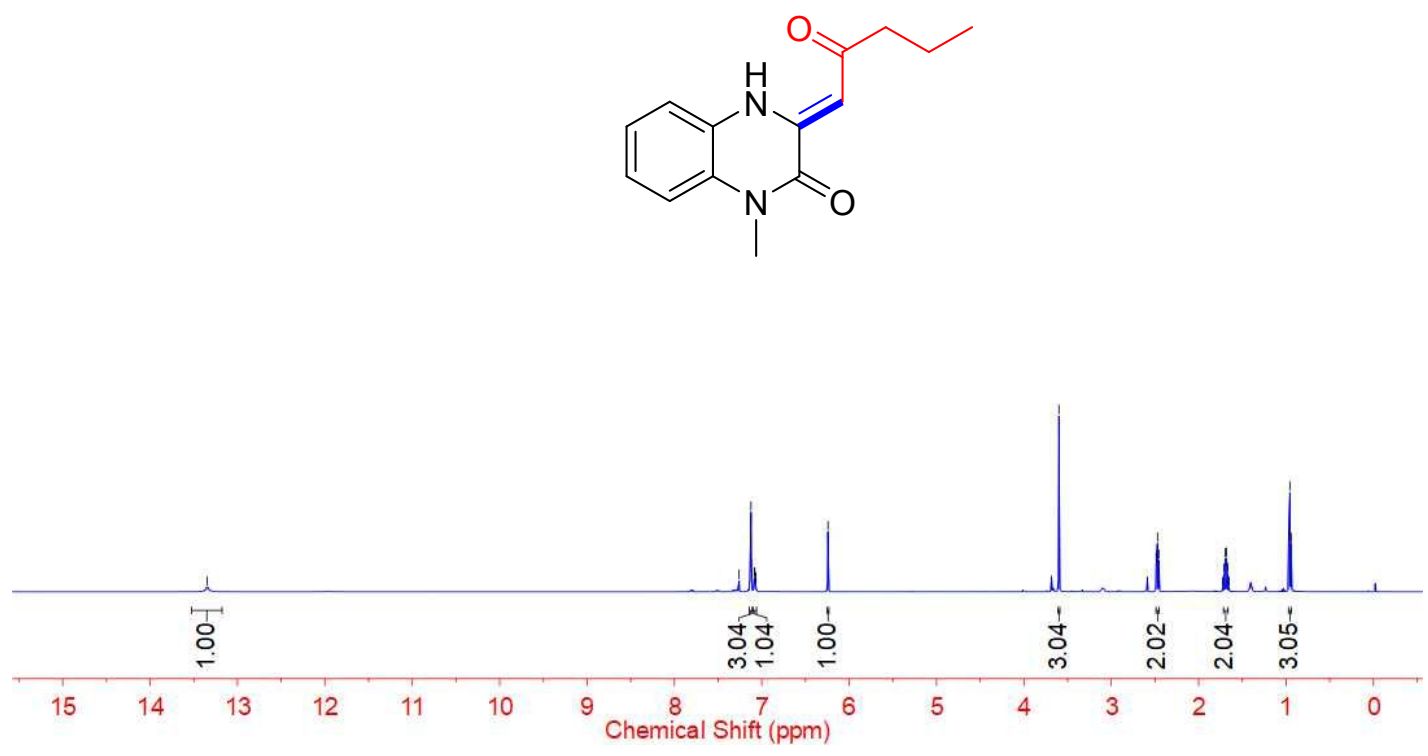

Figure S79. ${ }^{1} \mathrm{H} \mathrm{NMR}\left(500 \mathrm{MHz}, \mathrm{CDCl}_{3}\right)$ spectrum of 7.
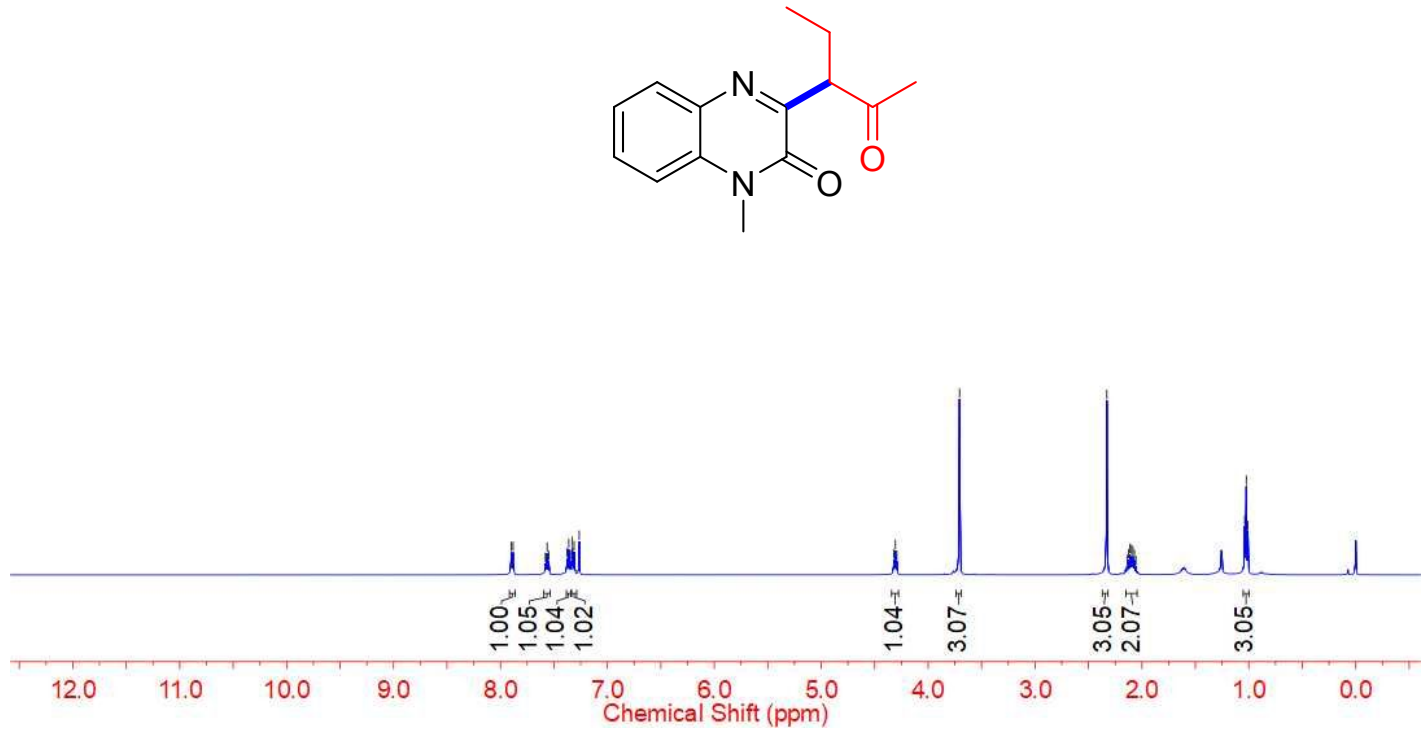

Figure S80. ${ }^{1} \mathrm{H}$ NMR $\left(500 \mathrm{MHz}, \mathrm{CDCl}_{3}\right)$ spectrum of 8. 


\section{Copies of HRMS Spectra}

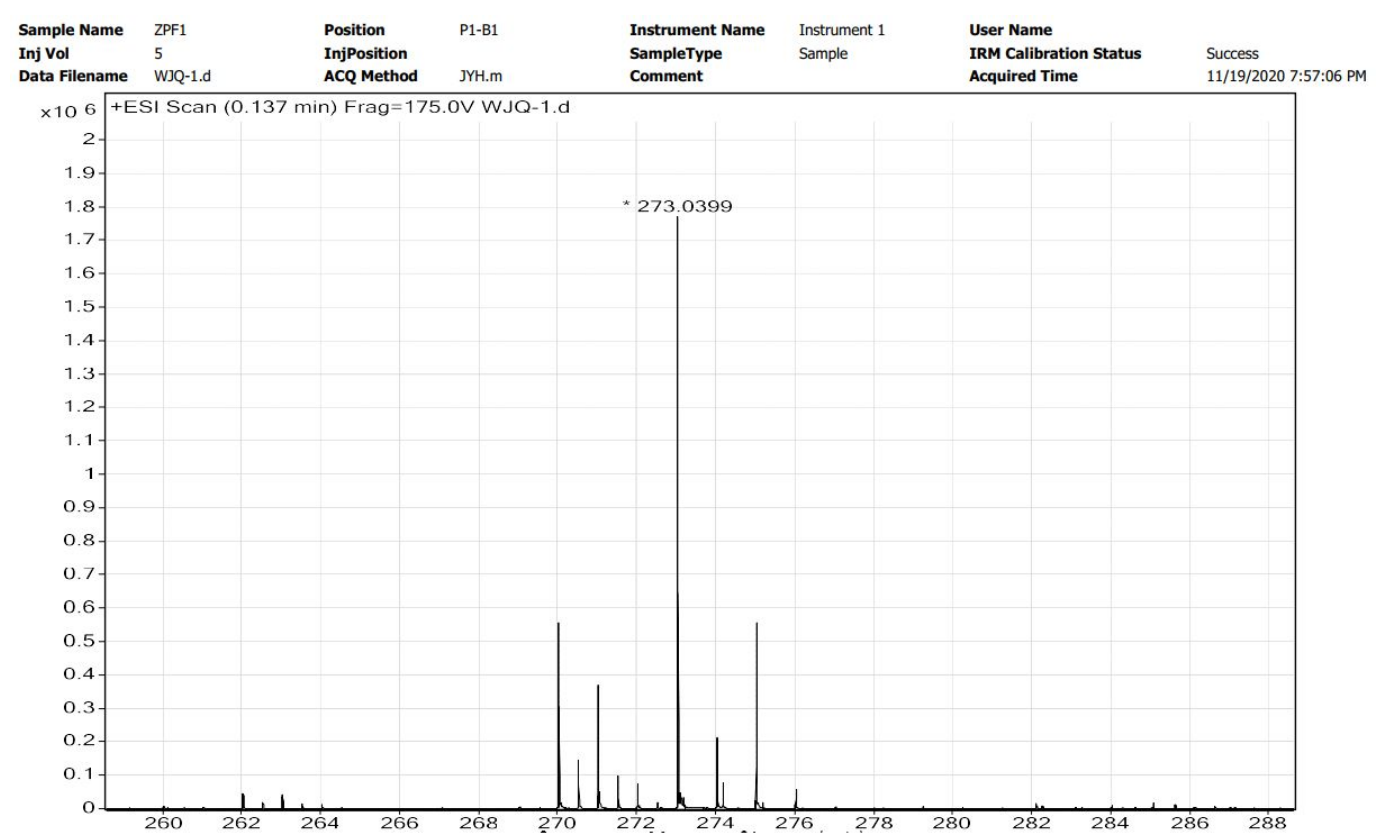

\begin{tabular}{|c|c|c|c|c|c|c|c|}
\hline Formula (M) & Score (MFG) & Mass & Mass (MFG) & $\mathrm{m} / \mathrm{z}($ Calc) & Diff (ppm) & DBE & $\mathrm{m} / \mathrm{z}$ \\
\hline $\mathrm{C}_{12} \mathrm{H}_{11} \mathrm{ClN}_{2} \mathrm{O}_{2}$ & 99.79 & 250.0507 & 250.0509 & 273.0401 & 0.91 & 8 & 273.0399 \\
\hline
\end{tabular}

Figure S81. HRMS spectrum of $\mathbf{3 a}$.

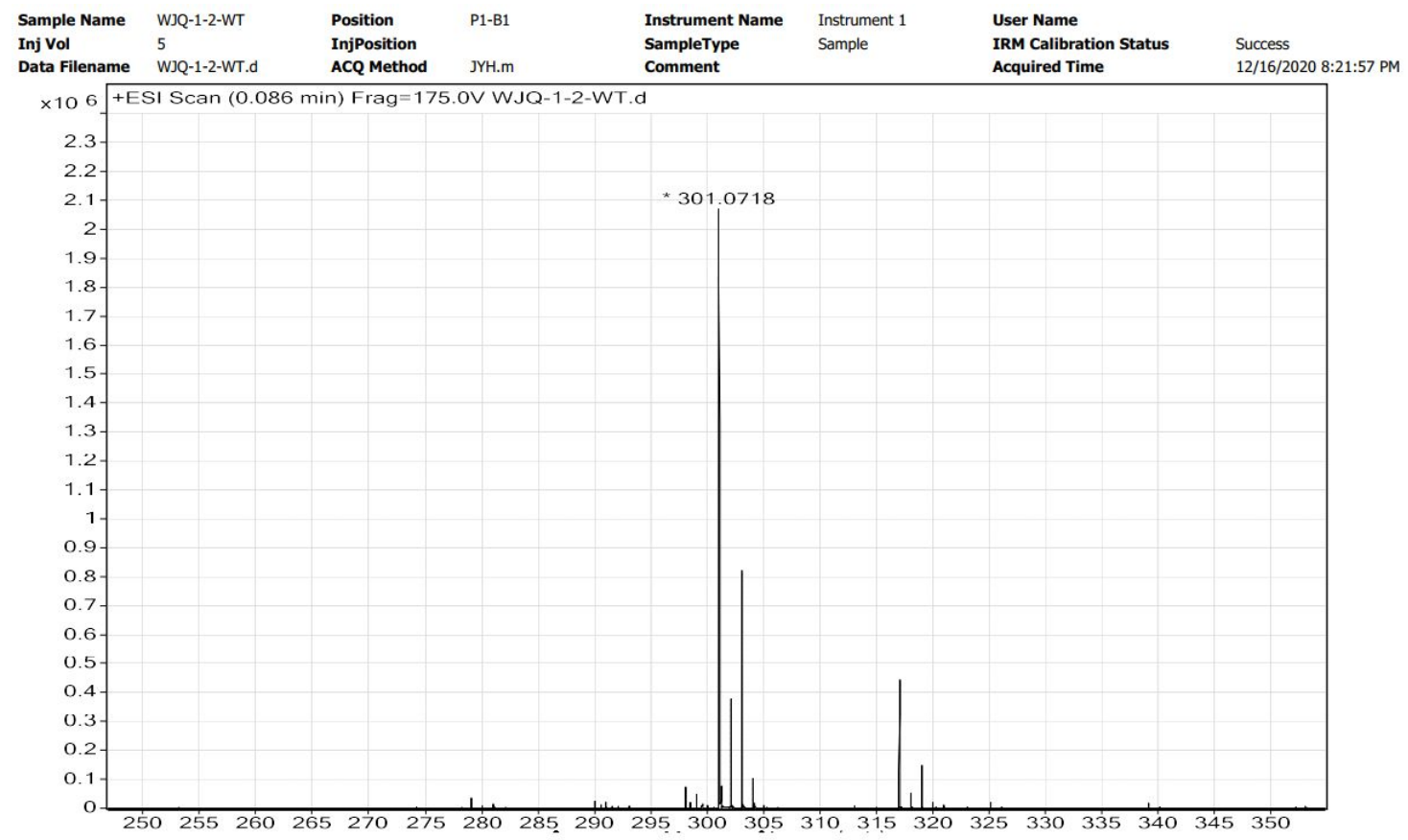

\begin{tabular}{|c|c|c|c|c|c|c|c|}
\hline Formula (M) & Score (MFG) & Mass & Mass (MFG) & $\mathrm{m} / \mathrm{z}($ Calc) & Diff (ppm) & DBE & $\mathrm{m} / \mathrm{z}$ \\
\hline $\mathrm{C}_{14} \mathrm{H}_{15} \mathrm{ClN}_{2} \mathrm{O}_{2}$ & 99.48 & 278.0826 & 278.0822 & 301.0714 & -1.34 & 8 & 301.0718 \\
\hline
\end{tabular}

Figure S82. HRMS spectrum of $\mathbf{3 b}$. 


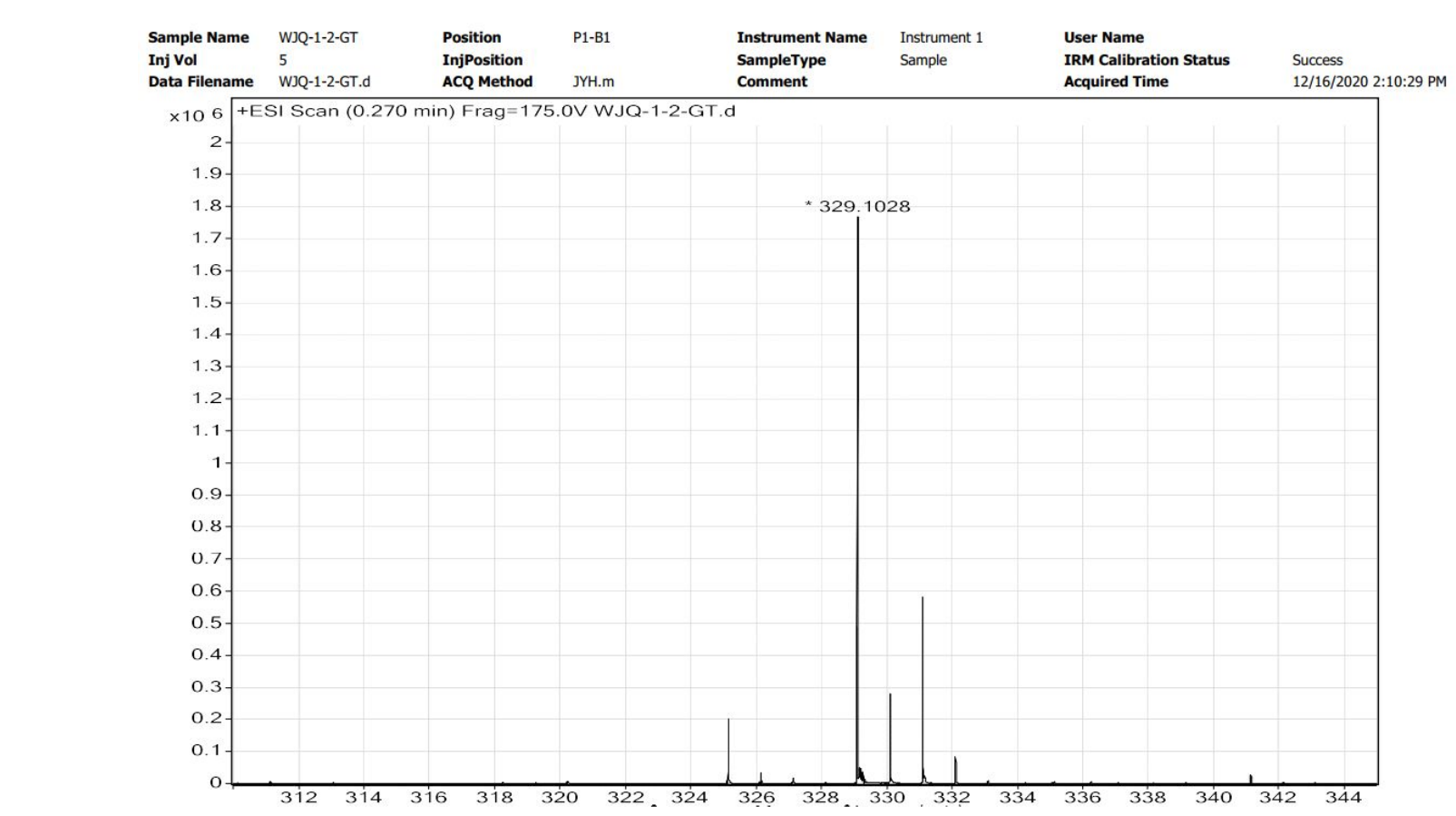

\begin{tabular}{|c|c|c|c|c|c|c|c|}
\hline Formula (M) & Score (MFG) & Mass & Mass (MFG) & $\mathrm{m} / \mathrm{z}(\mathrm{Calc})$ & Diff $(\mathrm{ppm})$ & DBE & $\mathrm{m} / \mathrm{z}$ \\
\hline $\mathrm{C}_{16} \mathrm{H}_{19} \mathrm{ClN}_{2} \mathrm{O}_{2}$ & 99.98 & 306.1136 & 306.1135 & 329.1027 & -0.24 & 8 & 329.1028 \\
\hline
\end{tabular}

Figure S83. HRMS spectrum of 3c.

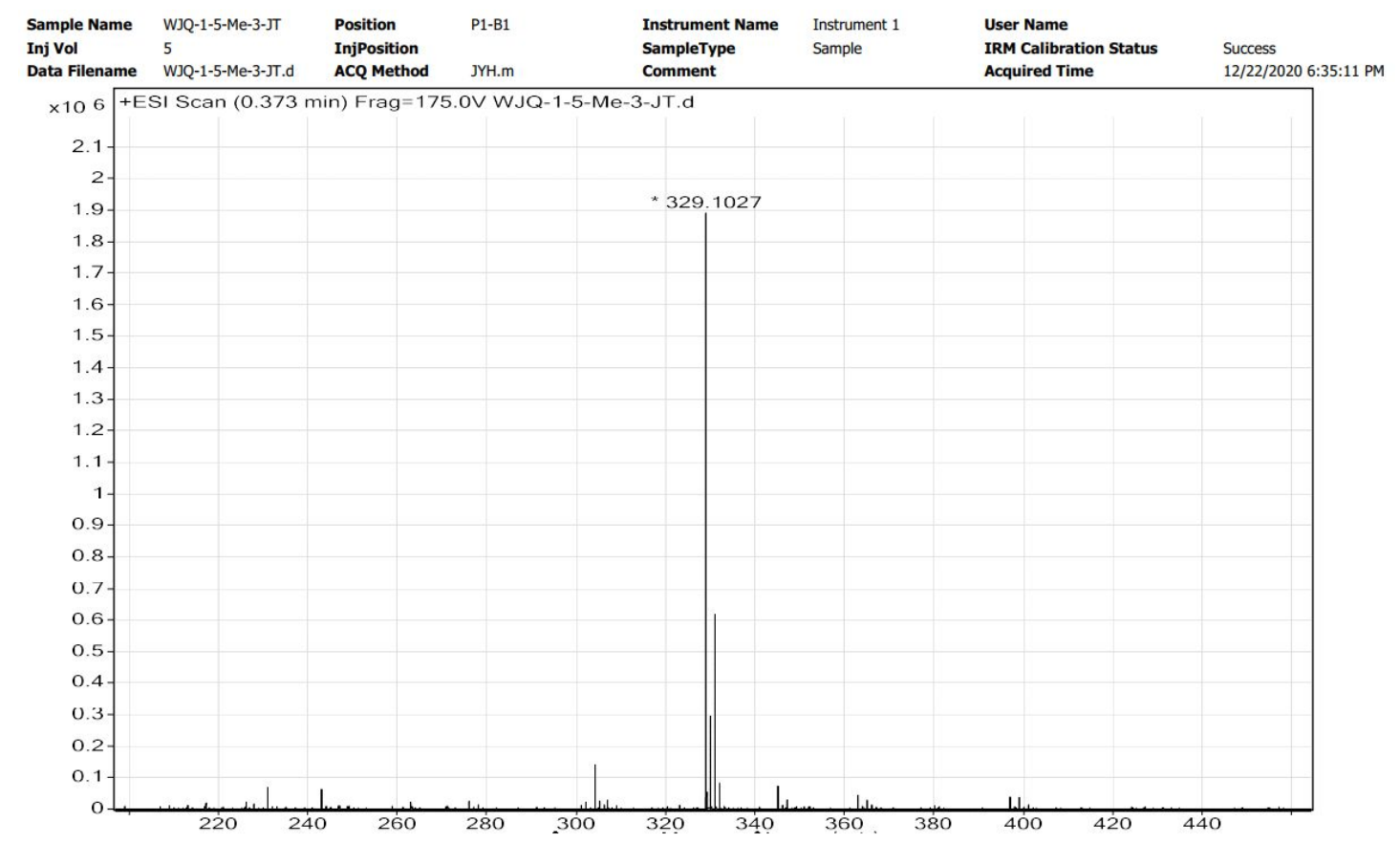

\begin{tabular}{|c|c|c|c|c|c|c|c|}
\hline Formula (M) & Score (MFG) & Mass & Mass (MFG) & $\mathrm{m} / \mathrm{z}($ Calc) & Diff (ppm) & DBE & $\mathrm{m} / \mathrm{z}$ \\
\hline $\mathrm{C}_{16} \mathrm{H}_{19} \mathrm{ClN}_{2} \mathrm{O}_{2}$ & 100 & 306.1135 & 306.1135 & 329.1027 & 0.09 & 8 & 329.1027 \\
\hline
\end{tabular}

Figure S84. HRMS spectrum of 3d. 


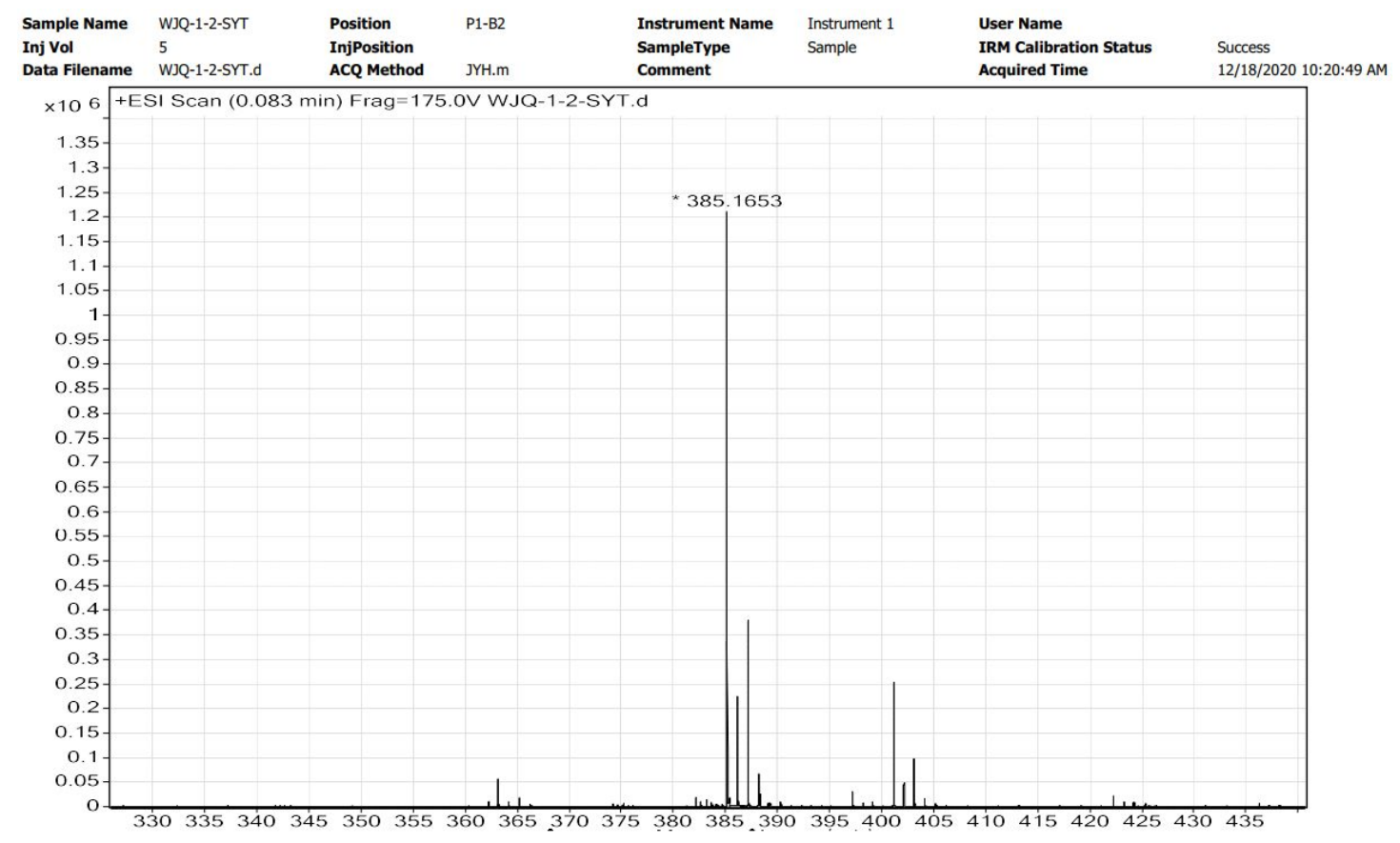

\begin{tabular}{|c|c|c|c|c|c|c|c|}
\hline Formula (M) & Score (MFG) & Mass & Mass (MFG) & $\mathrm{m} / \mathrm{z}($ Calc) & Diff (ppm) & DBE & $\mathrm{m} / \mathrm{z}$ \\
\hline $\mathrm{C}_{20} \mathrm{H}_{27} \mathrm{ClN}_{2} \mathrm{O}_{2}$ & 100 & 362.1761 & 362.1761 & 385.1653 & 0.07 & 8 & 385.1653 \\
\hline
\end{tabular}

Figure S85. HRMS spectrum of $\mathbf{3 e}$.

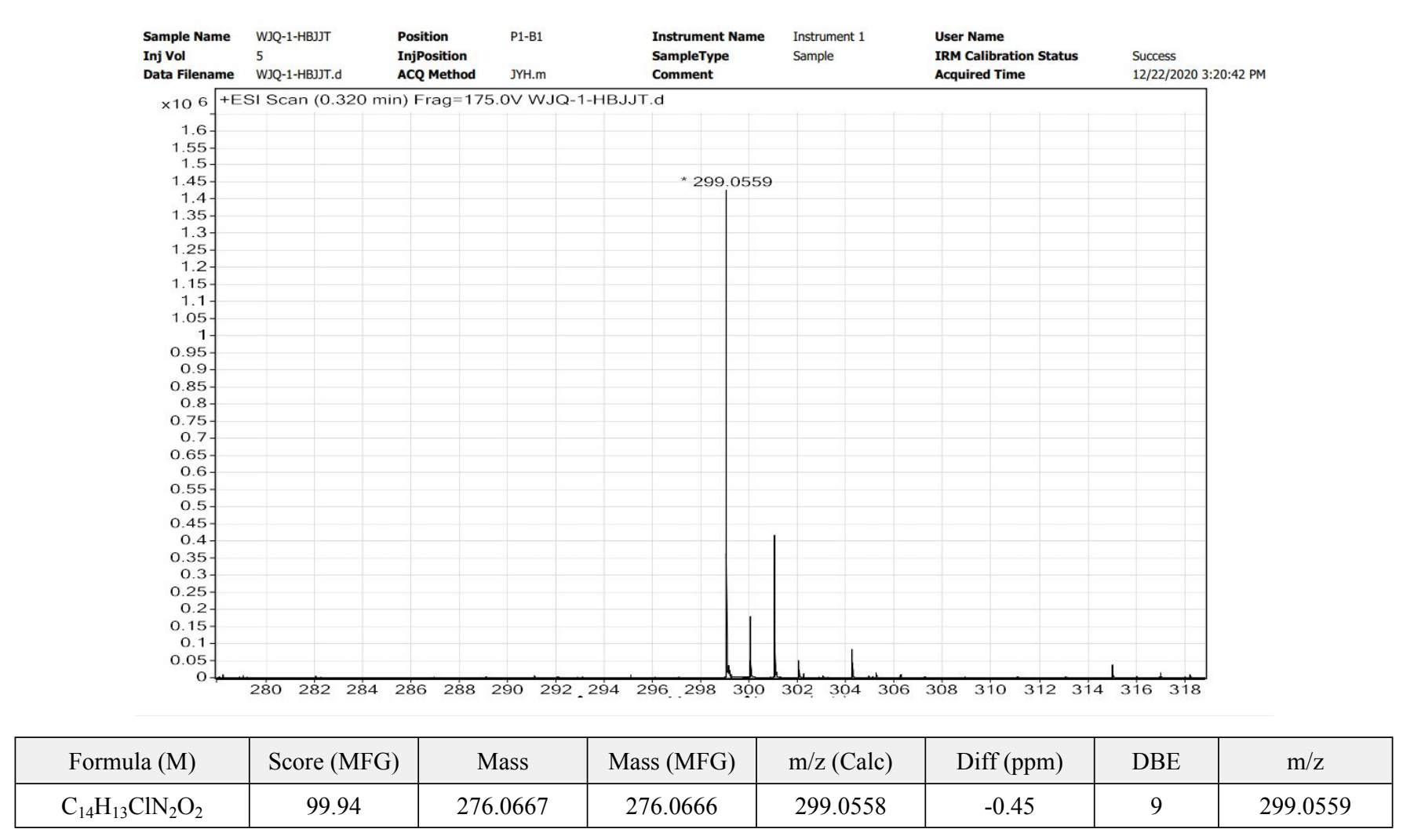

Figure S86. HRMS spectrum of $\mathbf{3 f}$. 


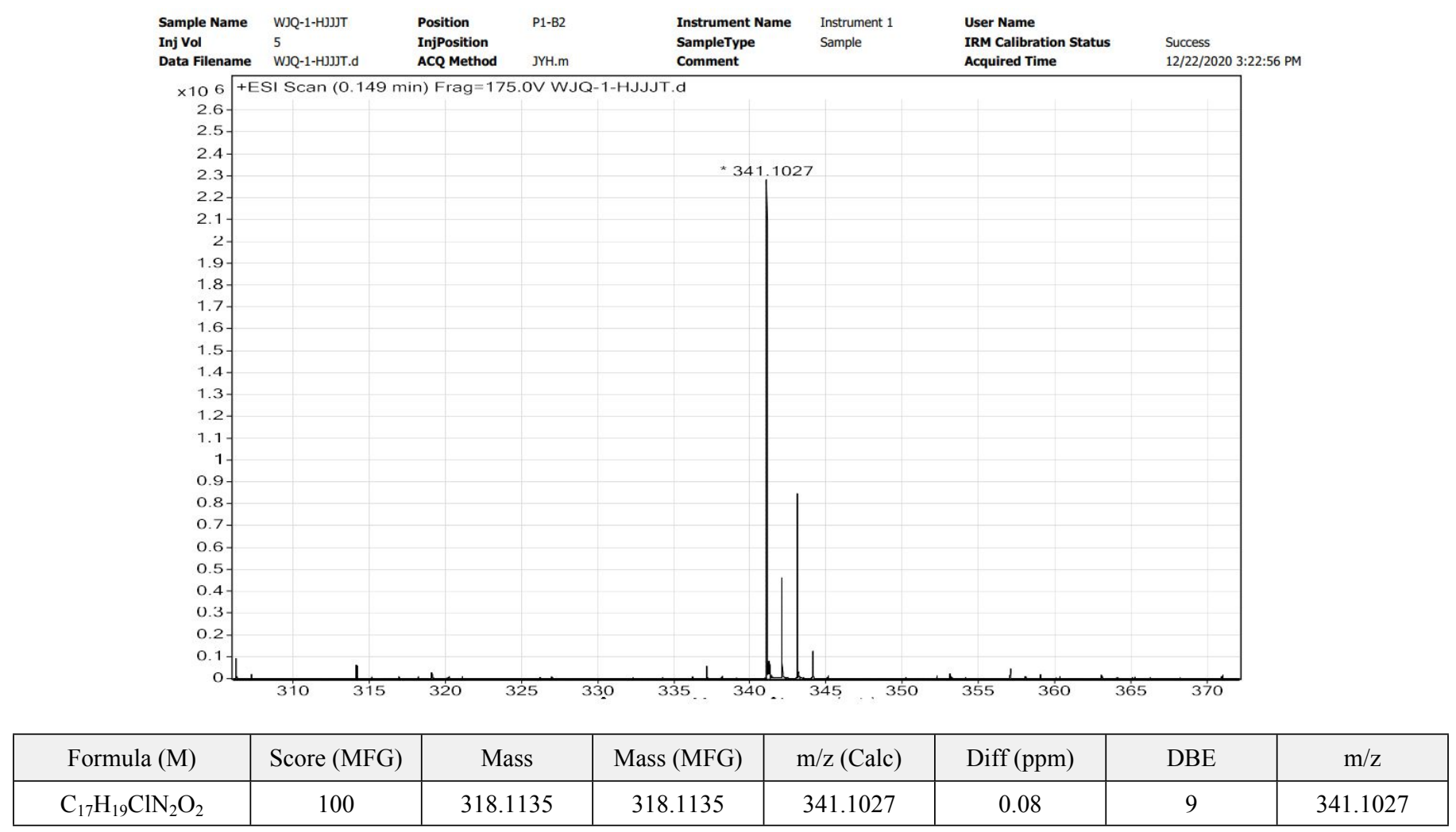

Figure S87. HRMS spectrum of $\mathbf{3 g}$.

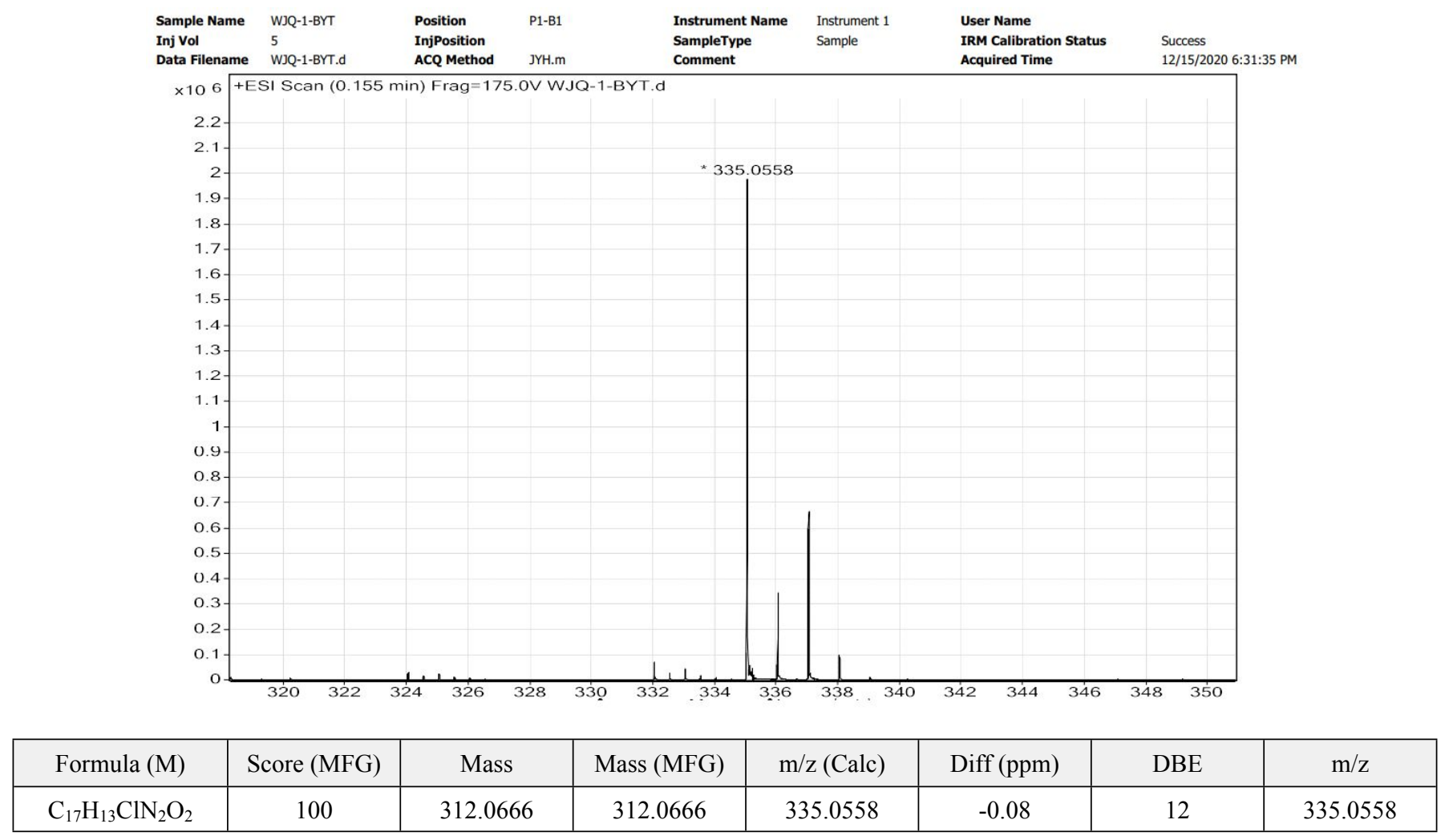

Figure S88. HRMS spectrum of $\mathbf{3 h}$. 


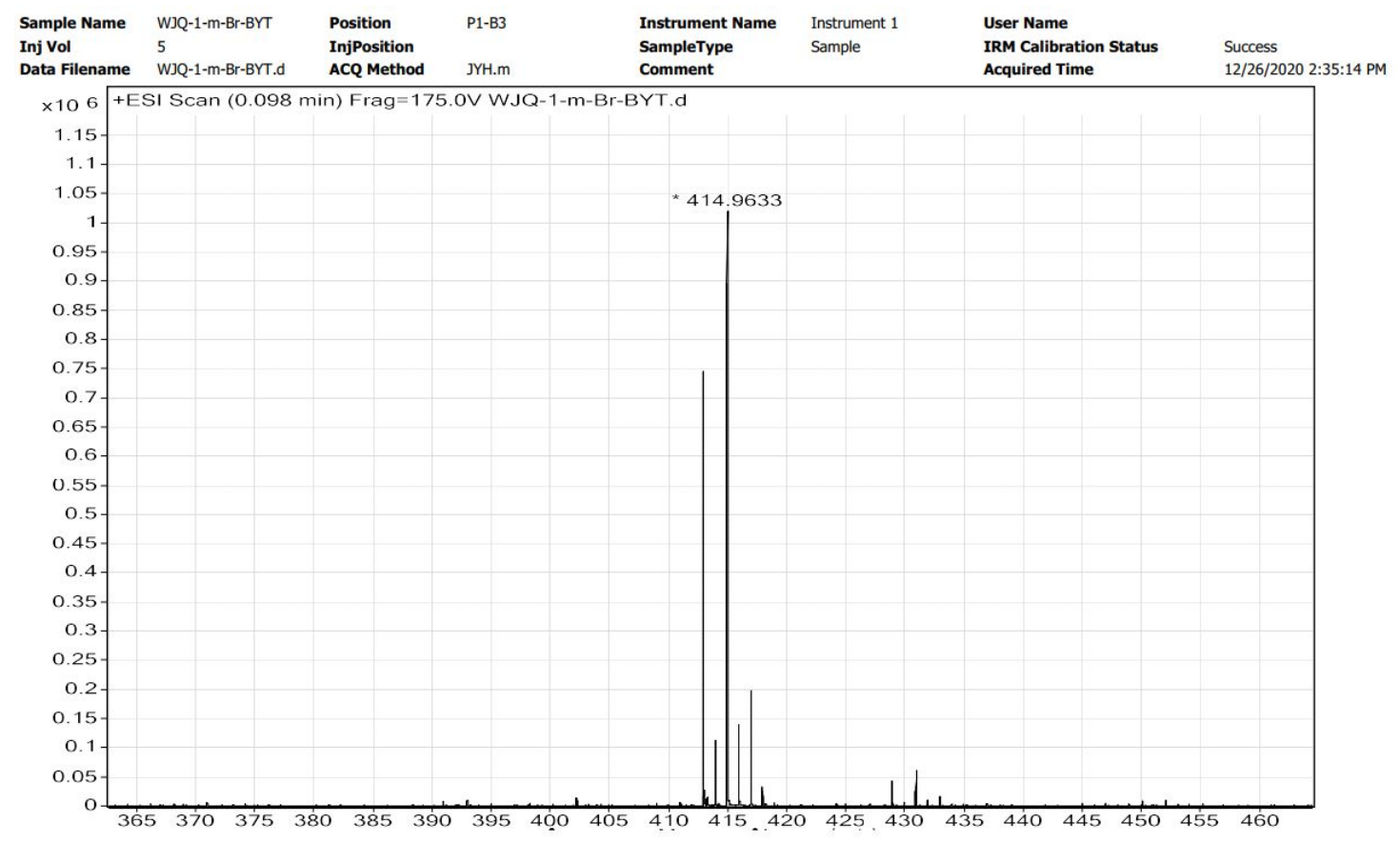

\begin{tabular}{|c|c|c|c|c|c|c|c|}
\hline Formula (M) & Score (MFG) & Mass & Mass (MFG) & $\mathrm{m} / \mathrm{z}($ Calc) & Diff (ppm) & DBE & $\mathrm{m} / \mathrm{z}$ \\
\hline $\mathrm{C}_{17} \mathrm{H}_{12} \mathrm{BrClN}_{2} \mathrm{O}_{2}$ & 98.33 & 389.9763 & 389.9771 & 412.9663 & 2.02 & 12 & 412.9655 \\
\hline
\end{tabular}

Figure S89. HRMS spectrum of $\mathbf{3 i}$.

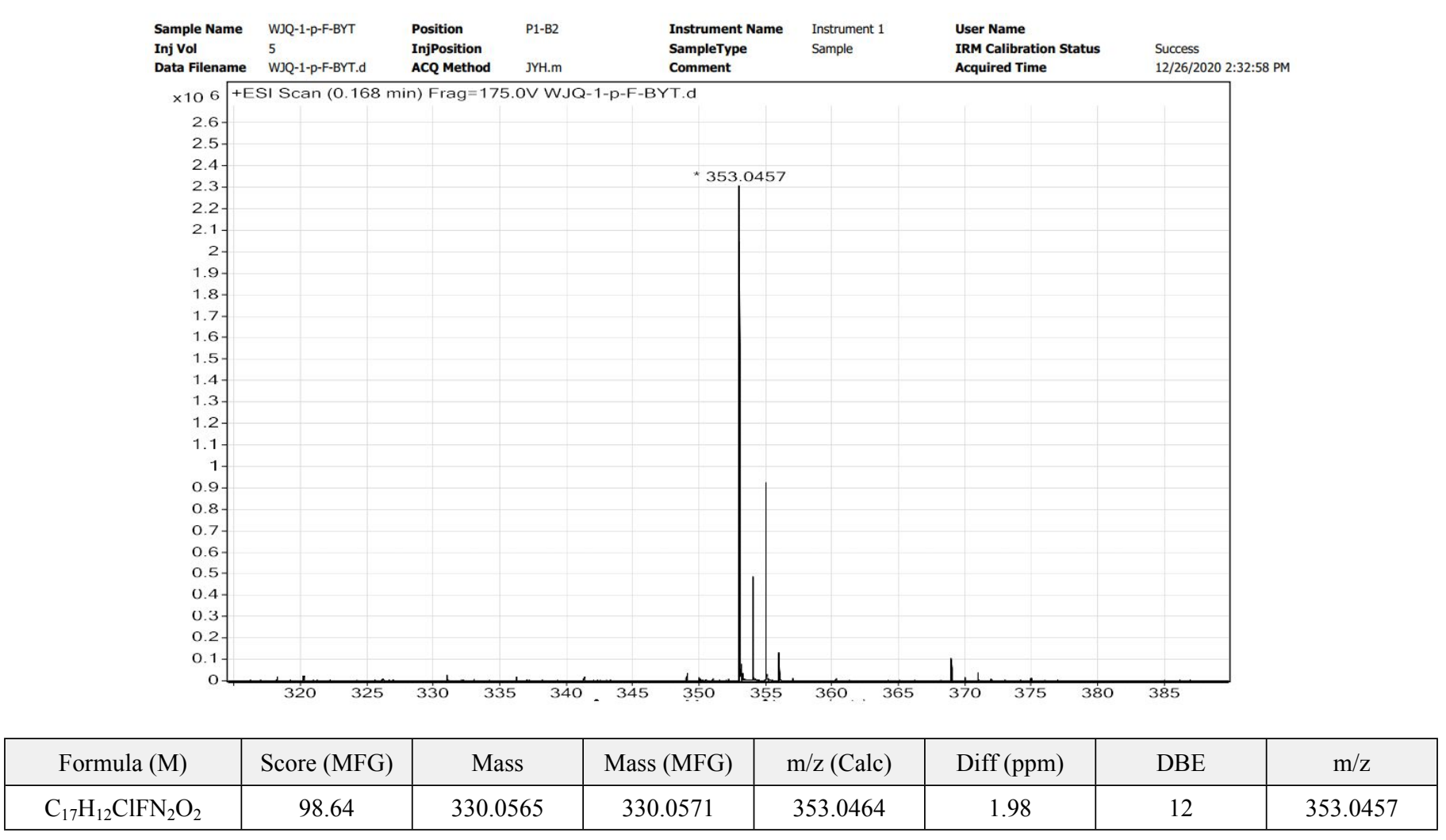

Figure S90. HRMS spectrum of $\mathbf{3 j}$. 


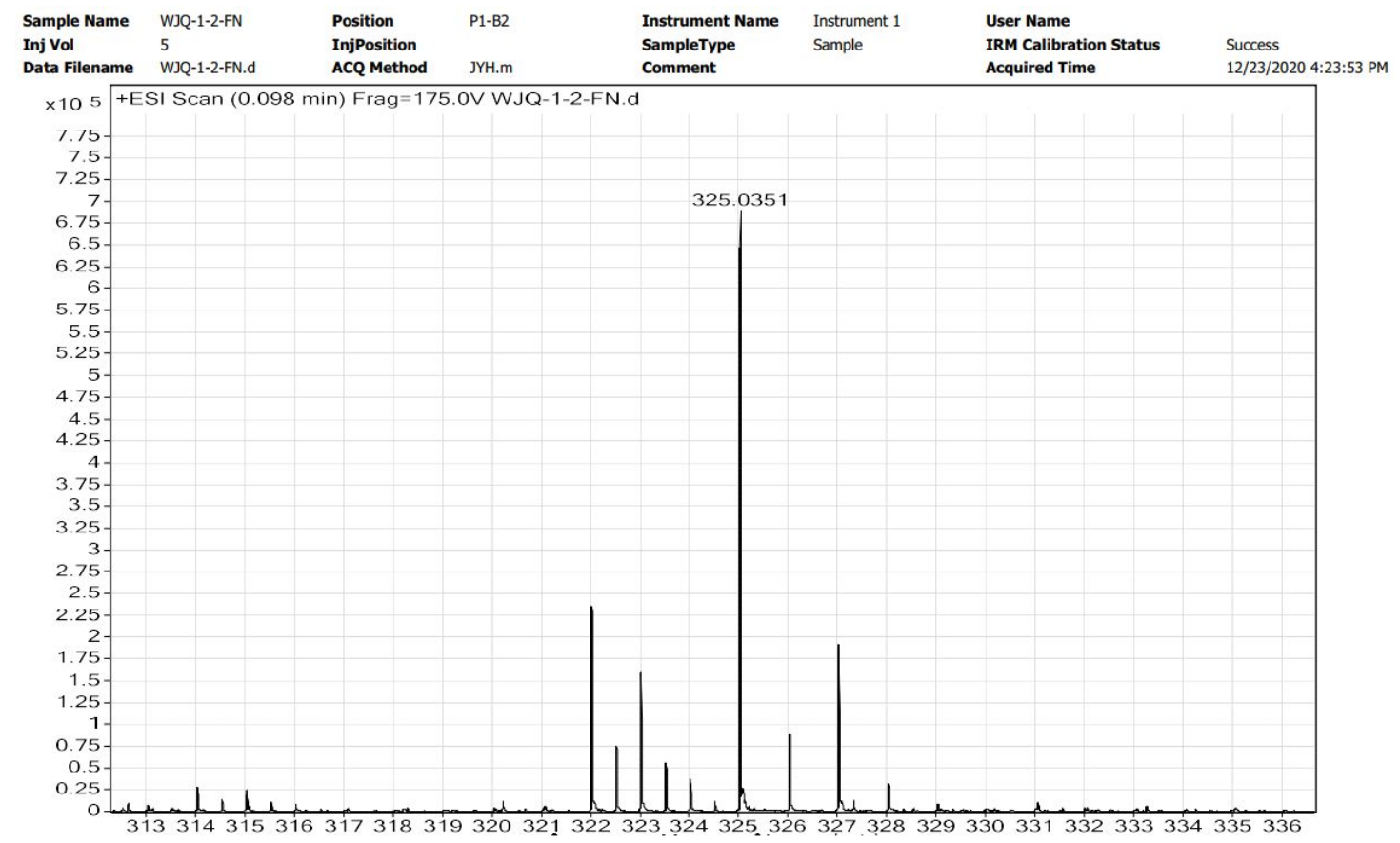

\begin{tabular}{|c|c|c|c|c|c|c|c|}
\hline Formula (M) & Score (MFG) & Mass & Mass (MFG) & $\mathrm{m} / \mathrm{z}($ Calc) & Diff (ppm) & DBE & $\mathrm{m} / \mathrm{z}$ \\
\hline $\mathrm{C}_{15} \mathrm{H}_{11} \mathrm{ClN}_{2} \mathrm{O}_{3}$ & 99.99 & 302.0459 & 302.0458 & 325.035 & -0.2 & 11 & 325.0351 \\
\hline
\end{tabular}

Figure S91. HRMS spectrum of $\mathbf{3 k}$.

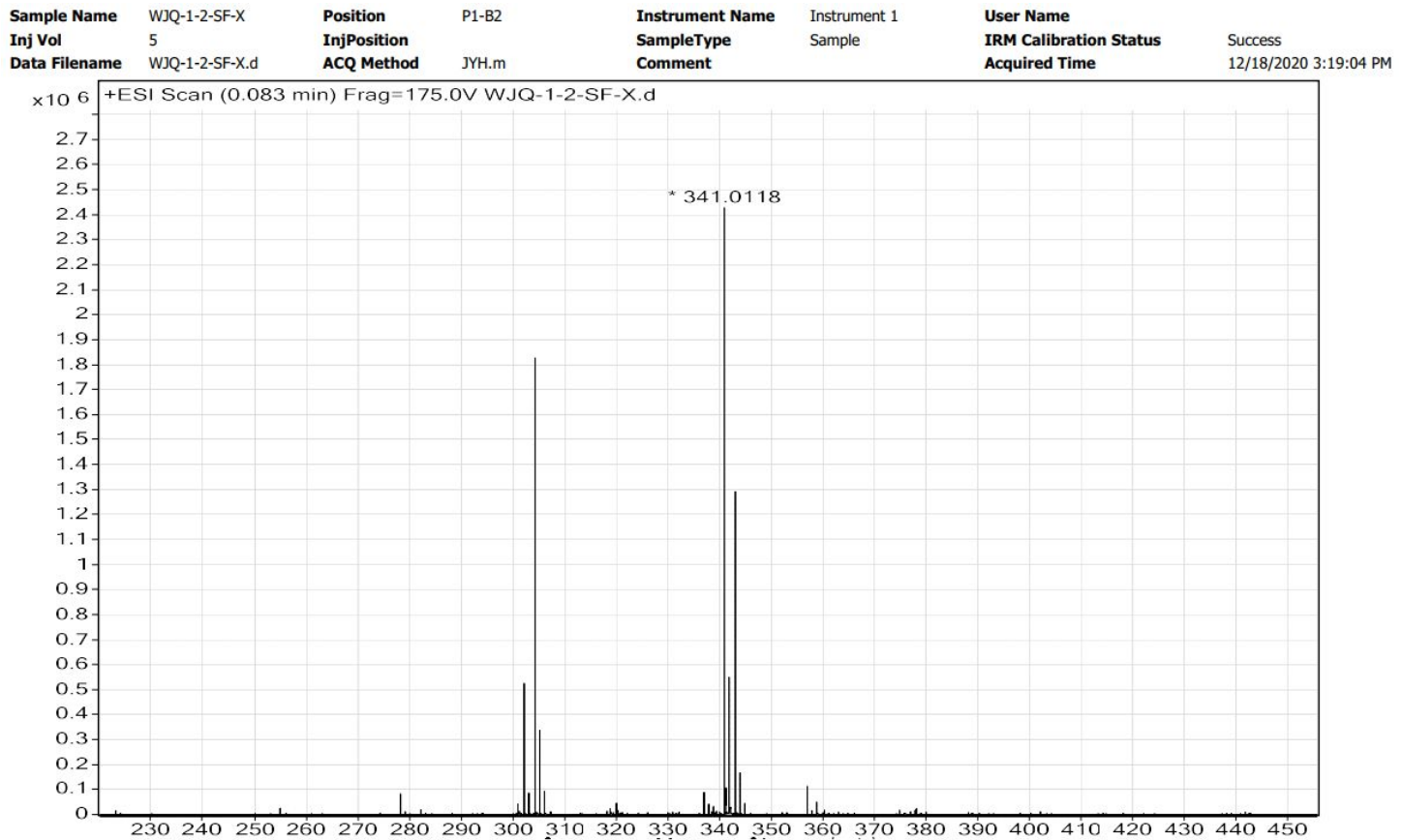

\begin{tabular}{|c|c|c|c|c|c|c|c|}
\hline Formula (M) & Score (MFG) & Mass & Mass (MFG) & $\mathrm{m} / \mathrm{z}($ Calc) & Diff $(\mathrm{ppm})$ & DBE & $\mathrm{m} / \mathrm{z}$ \\
\hline $\mathrm{C}_{15} \mathrm{H}_{11} \mathrm{ClN}_{2} \mathrm{O}_{2} \mathrm{~S}$ & 99.48 & 318.0226 & 318.023 & 341.0122 & 1.25 & 11 & 341.0118 \\
\hline
\end{tabular}

Figure S92. HRMS spectrum of $\mathbf{3 1}$. 


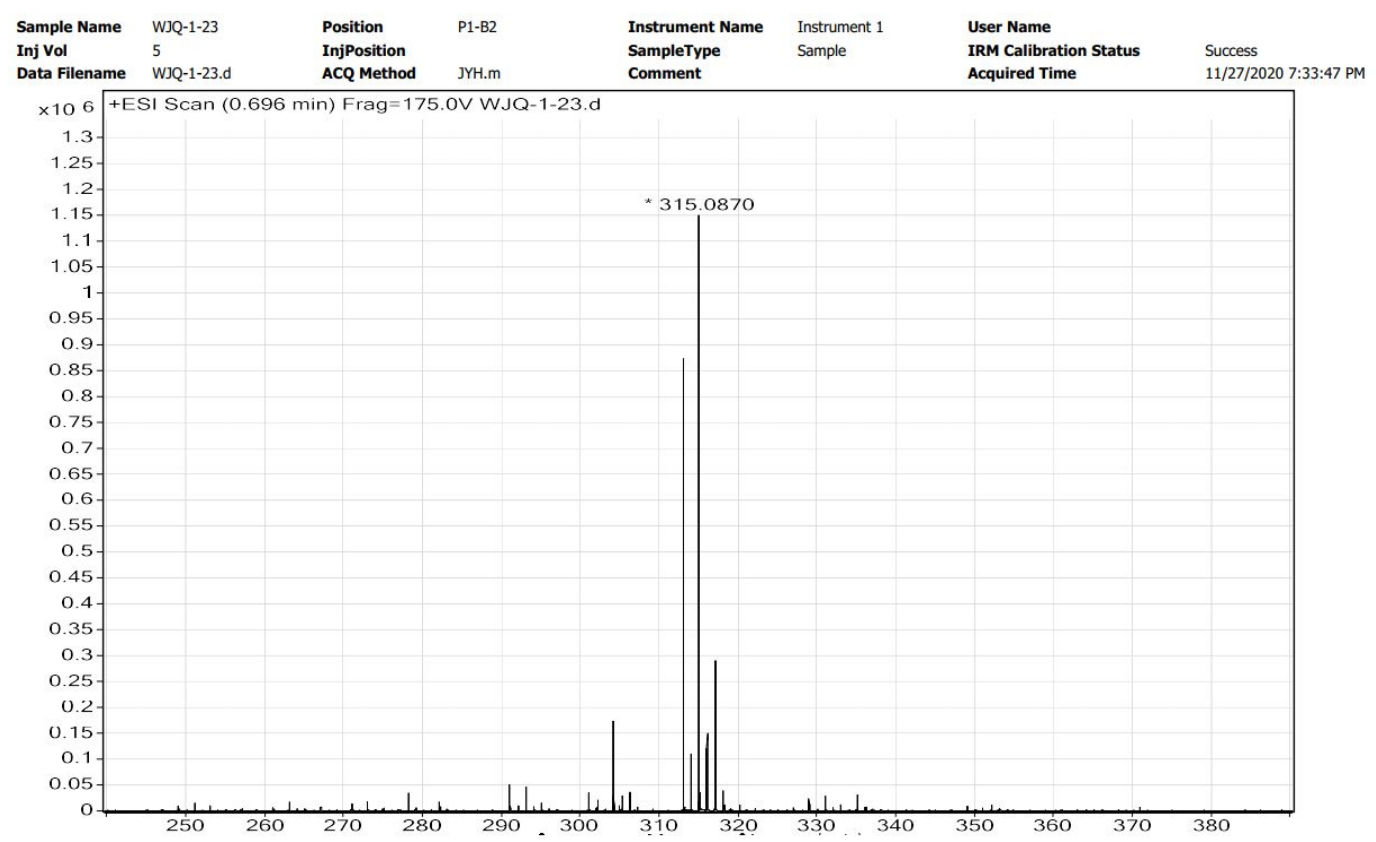

\begin{tabular}{|c|c|c|c|c|c|c|c|}
\hline Formula (M) & Score (MFG) & Mass & Mass (MFG) & $\mathrm{m} / \mathrm{z}($ Calc) & Diff (ppm) & DBE & $\mathrm{m} / \mathrm{z}$ \\
\hline $\mathrm{C}_{15} \mathrm{H}_{17} \mathrm{ClN}_{2} \mathrm{O}_{2}$ & 99.98 & 292.0978 & 292.0979 & 315.0871 & 0.26 & 8 & 315.0870 \\
\hline
\end{tabular}

Figure S93. HRMS spectrum of $\mathbf{3 m}$.

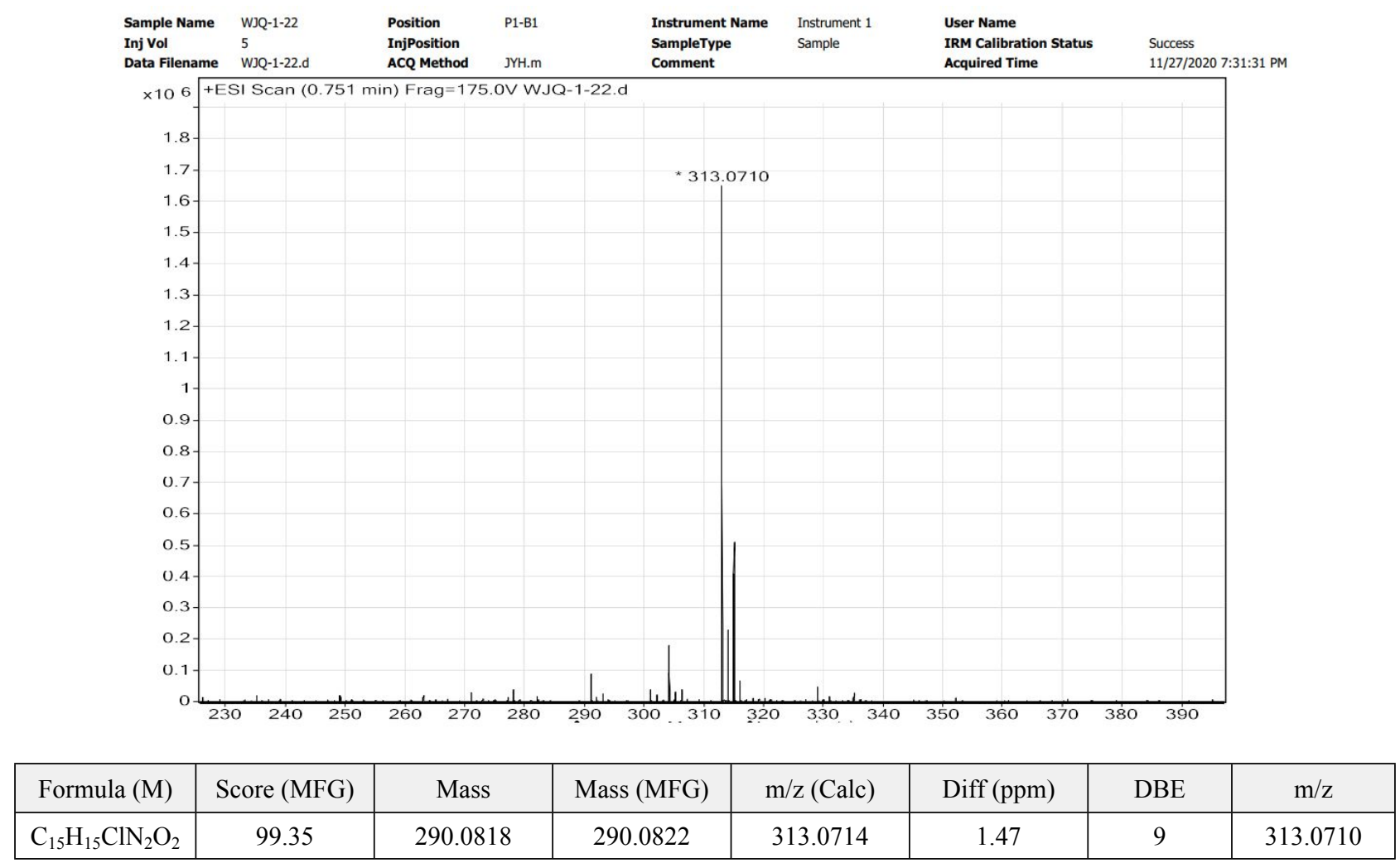

Figure S94. HRMS spectrum of $\mathbf{3 n}$. 


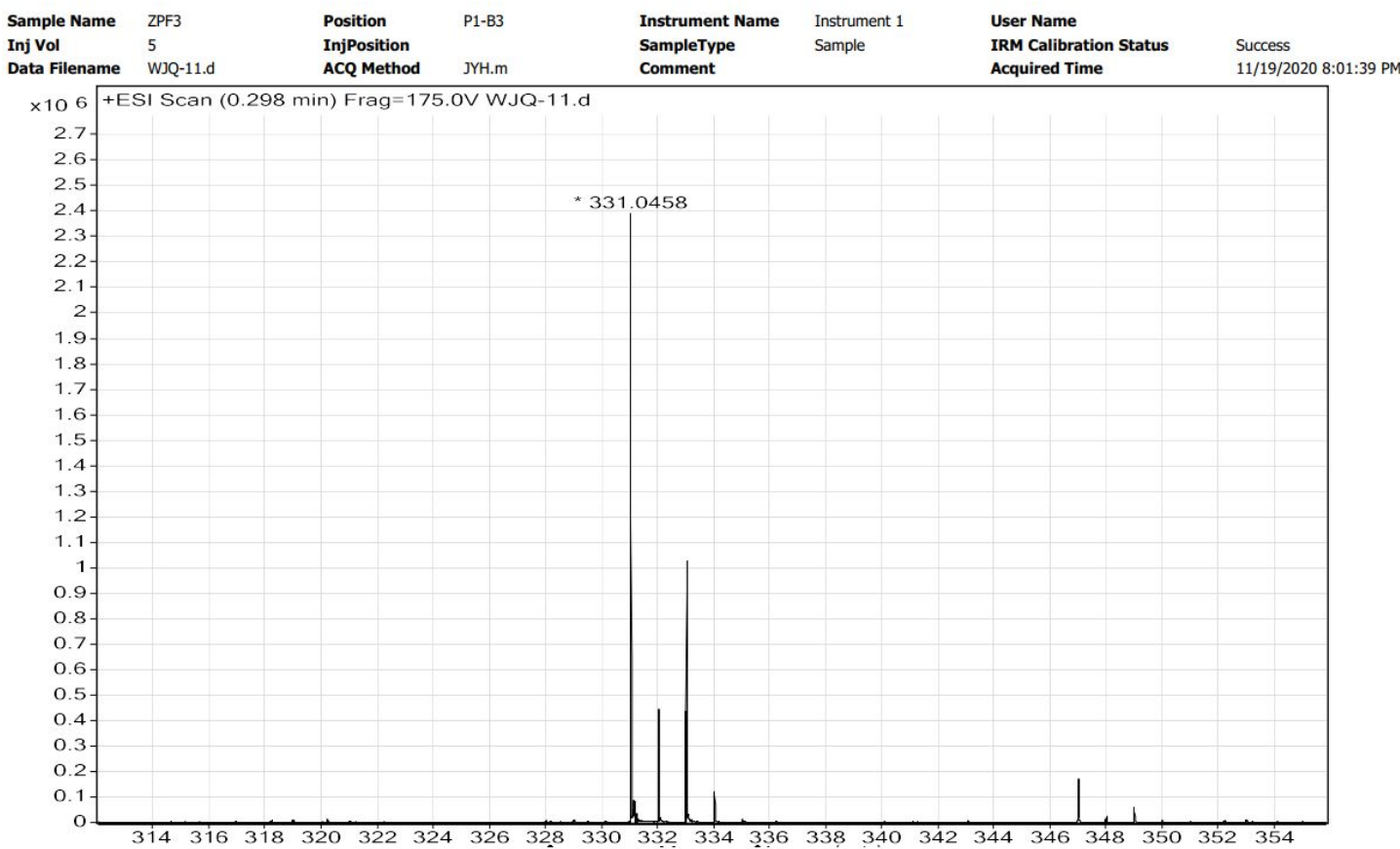

\begin{tabular}{|c|c|c|c|c|c|c|c|}
\hline Formula (M) & Score (MFG) & Mass & Mass (MFG) & $\mathrm{m} / \mathrm{z}(\mathrm{Calc})$ & Diff (ppm) & DBE & $\mathrm{m} / \mathrm{z}$ \\
\hline $\mathrm{C}_{14} \mathrm{H}_{13} \mathrm{ClN}_{2} \mathrm{O}_{4}$ & 99.87 & 308.0566 & 308.0564 & 331.0456 & -0.63 & 9 & 331.0458 \\
\hline
\end{tabular}

Figure S95. HRMS spectrum of $\mathbf{3 0 .}$

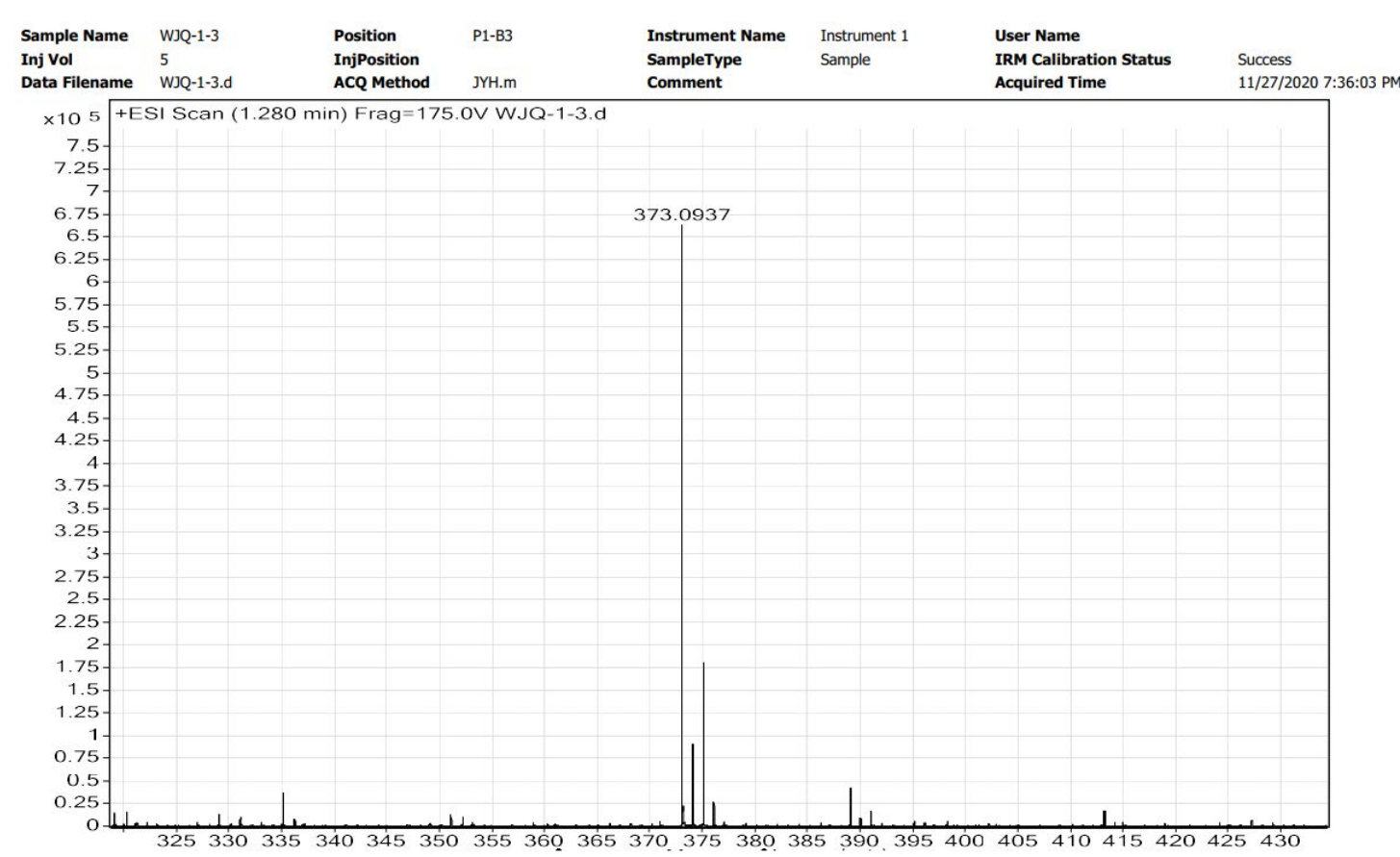

\begin{tabular}{|c|c|c|c|c|c|c|c|}
\hline Formula (M) & Score (MFG) & Mass & Mass (MFG) & $\mathrm{m} / \mathrm{z}($ Calc) & Diff (ppm) & DBE & $\mathrm{m} / \mathrm{z}$ \\
\hline $\mathrm{C}_{17} \mathrm{H}_{19} \mathrm{ClN}_{2} \mathrm{O}_{4}$ & 96.19 & 350.1045 & 350.1033 & 373.0926 & -3.27 & 9 & 373.0937 \\
\hline
\end{tabular}

Figure S96. HRMS spectrum of 3p. 


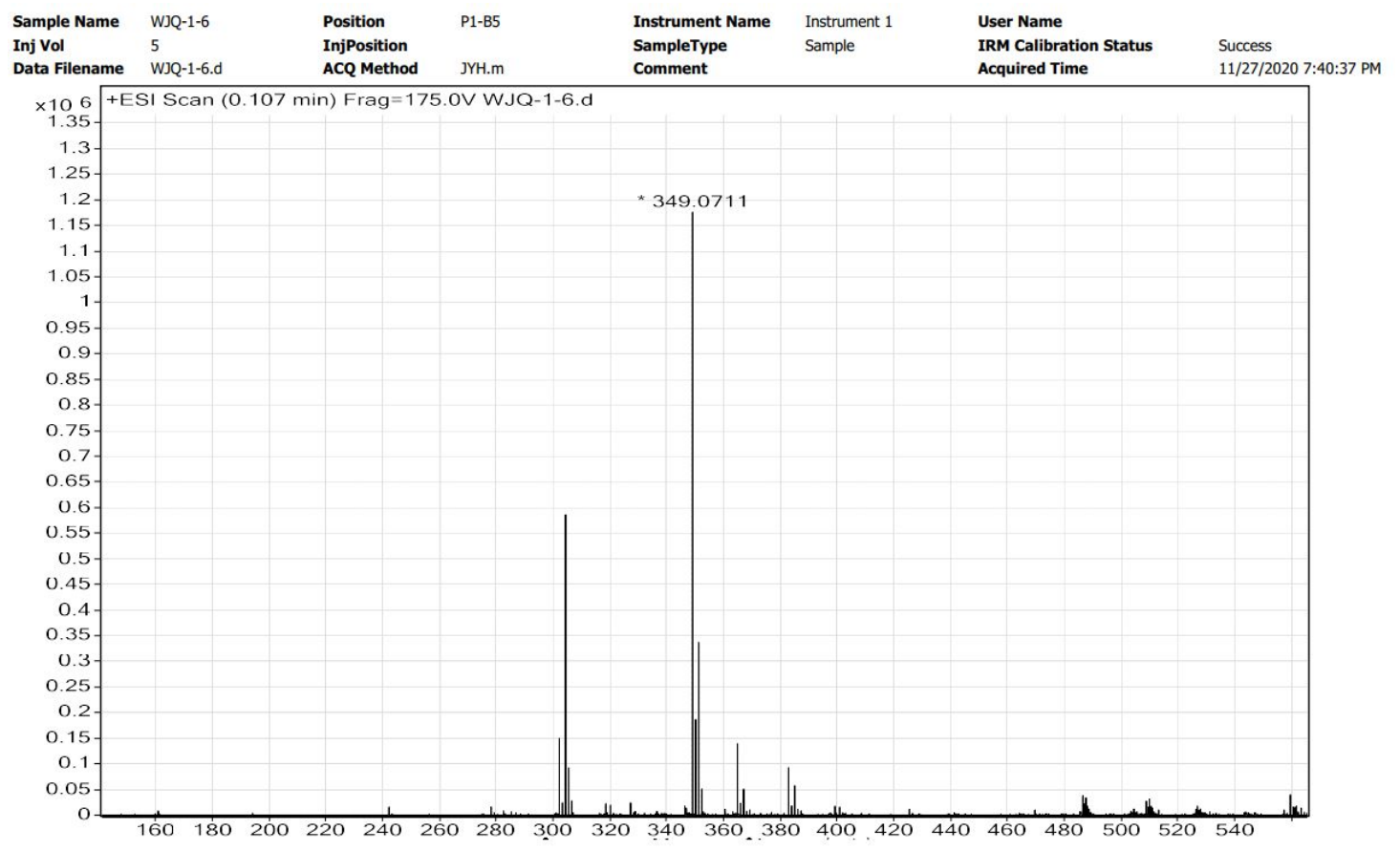

\begin{tabular}{|c|c|c|c|c|c|c|c|}
\hline Formula (M) & Score (MFG) & Mass & Mass (MFG) & $\mathrm{m} / \mathrm{z}($ Calc) & Diff (ppm) & DBE & $\mathrm{m} / \mathrm{z}$ \\
\hline $\mathrm{C}_{18} \mathrm{H}_{15} \mathrm{ClN}_{2} \mathrm{O}_{2}$ & 99.65 & 326.0819 & 326.0822 & 349.0714 & 1 & 12 & 349.0711 \\
\hline
\end{tabular}

Figure S97. HRMS spectrum of 3q.

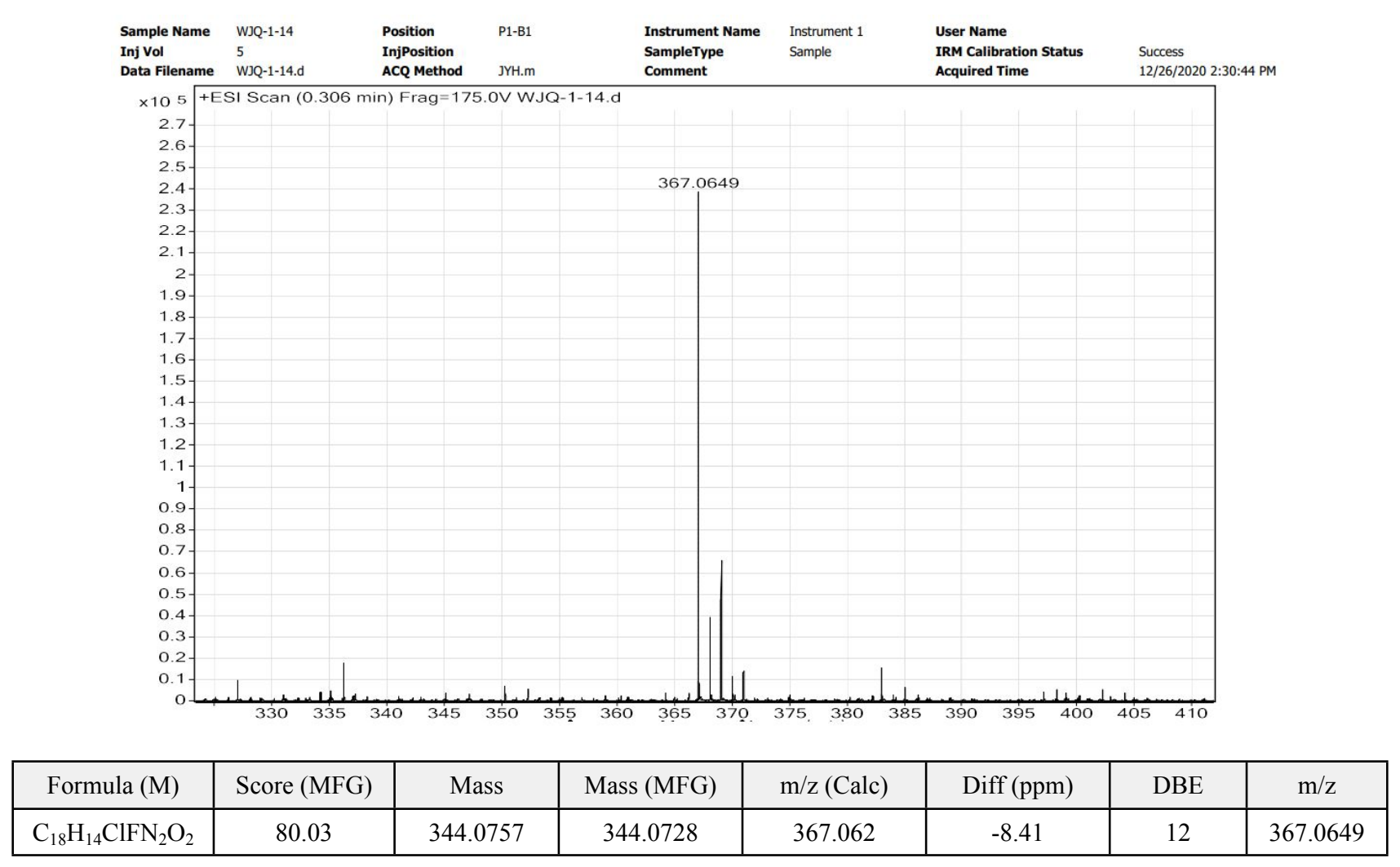

Figure S98. HRMS spectrum of 3r. 


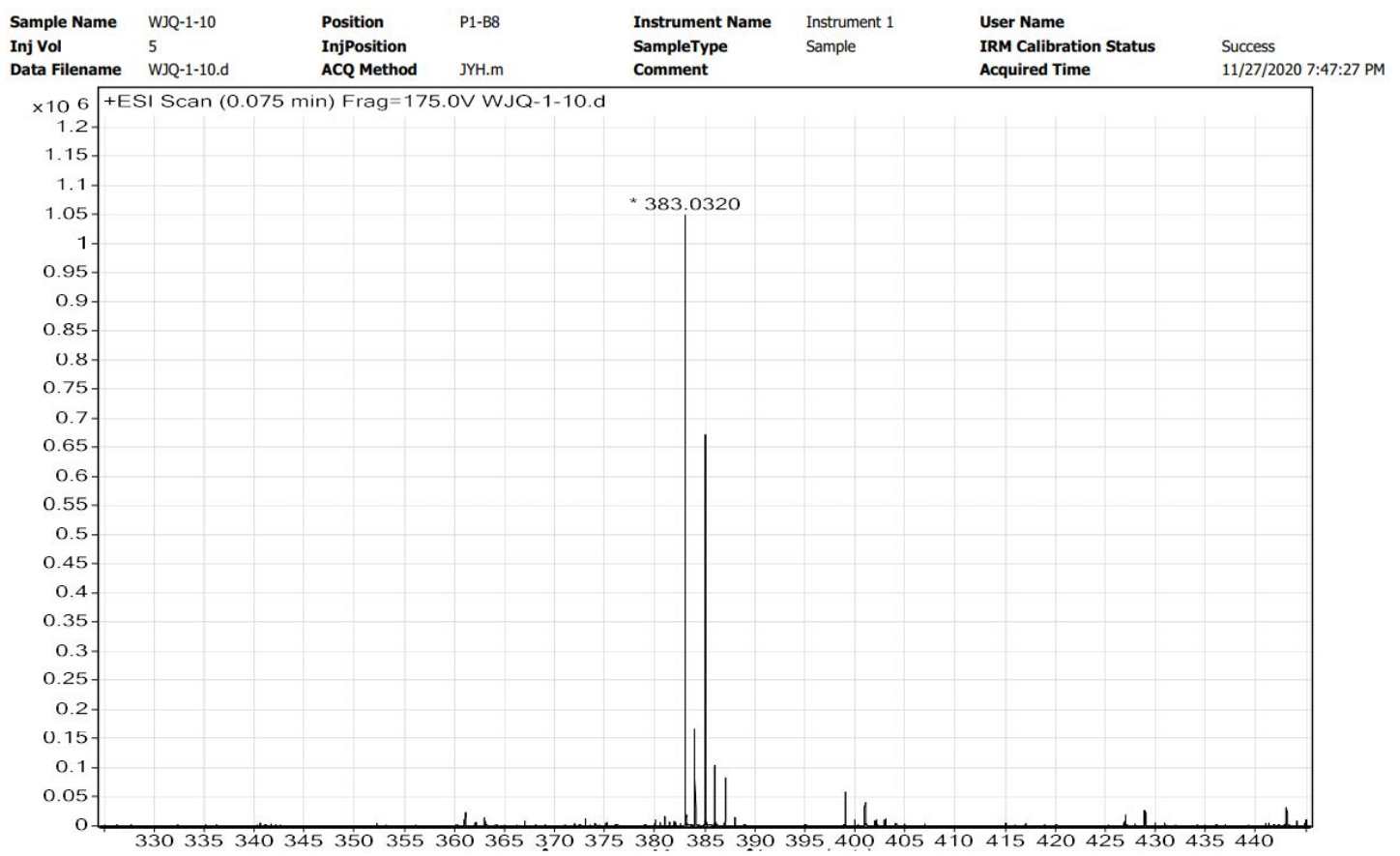

\begin{tabular}{|c|c|c|c|c|c|c|c|}
\hline Formula (M) & Score (MFG) & Mass & Mass (MFG) & $\mathrm{m} / \mathrm{z}($ Calc) & Diff (ppm) & DBE & $\mathrm{m} / \mathrm{z}$ \\
\hline $\mathrm{C}_{18} \mathrm{H}_{14} \mathrm{Cl}_{2} \mathrm{~N}_{2} \mathrm{O}_{2}$ & 99.39 & 360.0428 & 360.0432 & 383.0325 & 1.26 & 12 & 383.032 \\
\hline
\end{tabular}

Figure S99. HRMS spectrum of $\mathbf{3 s}$.

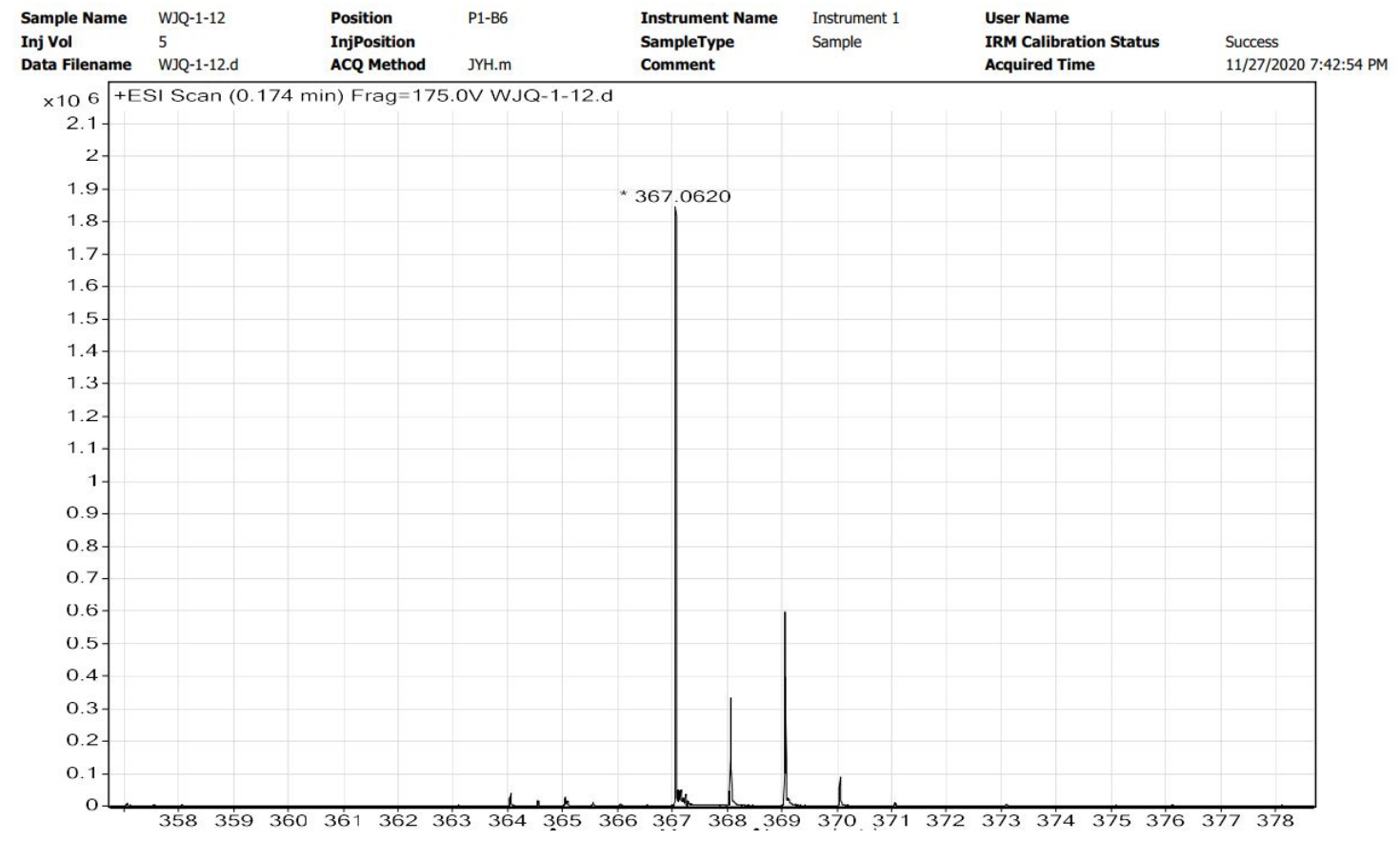

\begin{tabular}{|c|c|c|c|c|c|c|c|}
\hline Formula (M) & Score (MFG) & Mass & Mass (MFG) & $\mathrm{m} / \mathrm{z}(\mathrm{Calc})$ & Diff $(\mathrm{ppm})$ & DBE & $\mathrm{m} / \mathrm{z}$ \\
\hline $\mathrm{C}_{18} \mathrm{H}_{14} \mathrm{ClFN}_{2} \mathrm{O}_{2}$ & 100 & 344.0728 & 344.0728 & 367.0620 & 0.01 & 12 & 367.0620 \\
\hline
\end{tabular}

Figure S100. HRMS spectrum of $3 t$. 


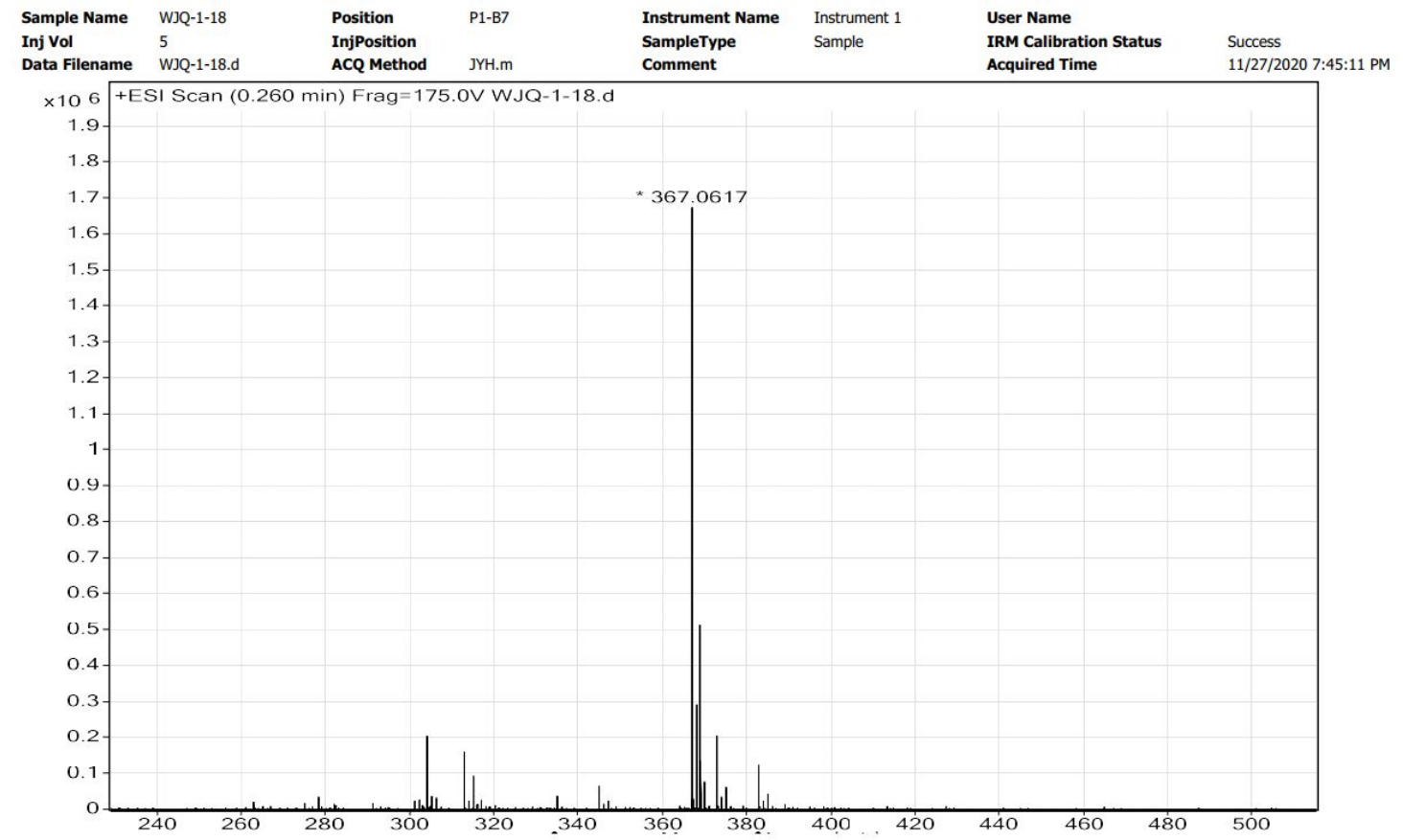

\begin{tabular}{|c|c|c|c|c|c|c|c|}
\hline Formula (M) & Score (MFG) & Mass & Mass (MFG) & $\mathrm{m} / \mathrm{z}($ Calc) & Diff (ppm) & DBE & $\mathrm{m} / \mathbf{z}$ \\
\hline $\mathrm{C}_{18} \mathrm{H}_{14} \mathrm{ClFN}_{2} \mathrm{O}_{2}$ & 99.71 & 344.0725 & 344.0728 & 367.0620 & 0.89 & 12 & 367.0617 \\
\hline
\end{tabular}

Figure S101. HRMS spectrum of $\mathbf{3 u}$.

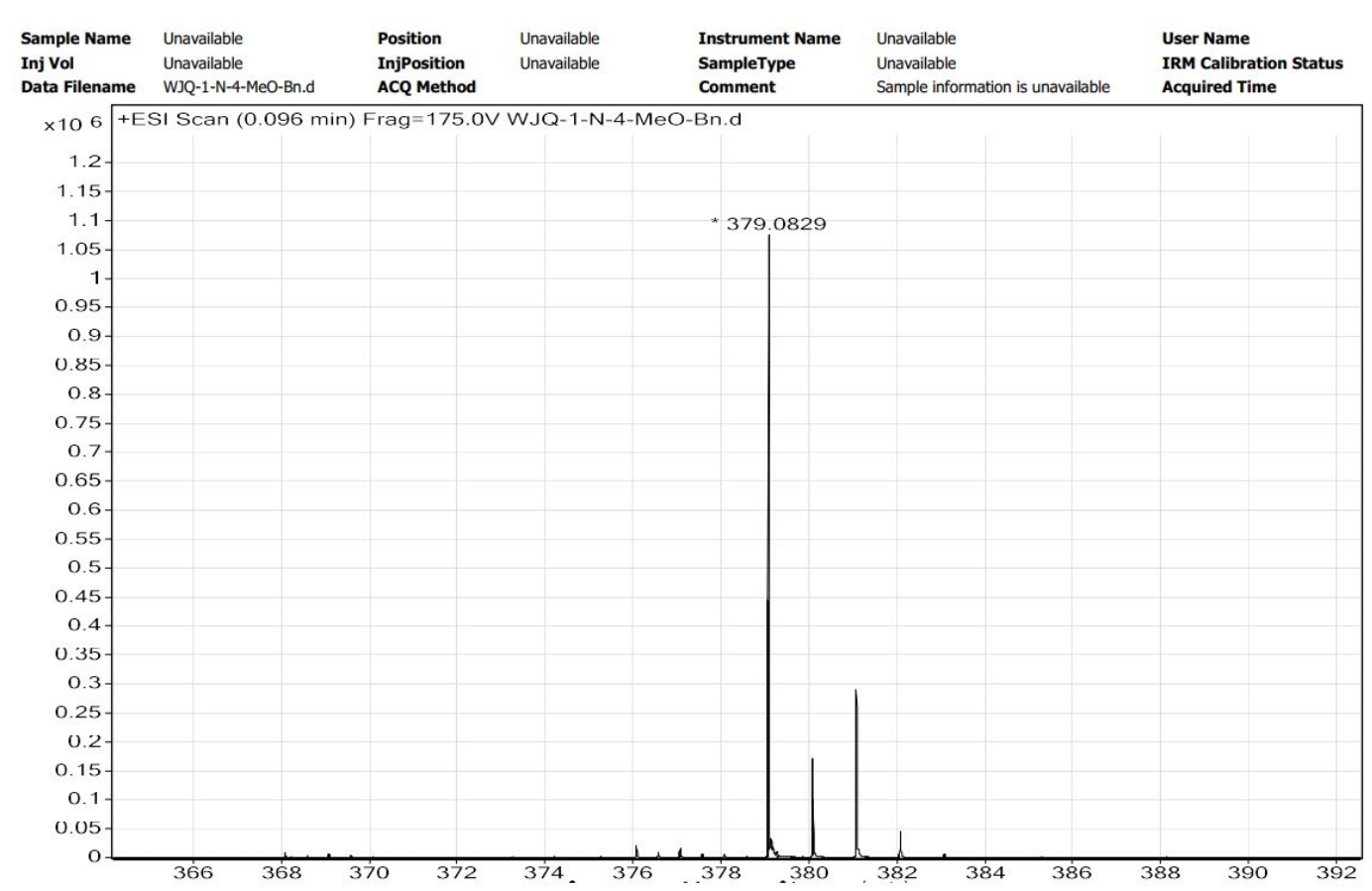

\begin{tabular}{|c|c|c|c|c|c|c|c|}
\hline Formula (M) & Score (MFG) & Mass & Mass (MFG) & $\mathrm{m} / \mathrm{z}(\mathrm{Calc})$ & Diff $(\mathrm{ppm})$ & DBE & $\mathrm{m} / \mathrm{z}$ \\
\hline $\mathrm{C}_{19} \mathrm{H}_{17} \mathrm{ClN}_{2} \mathrm{O}_{3}$ & 97.6 & 356.0937 & 356.0928 & 379.0820 & -2.55 & 12 & 379.0829 \\
\hline
\end{tabular}

Figure S102. HRMS spectrum of $\mathbf{3 v}$. 


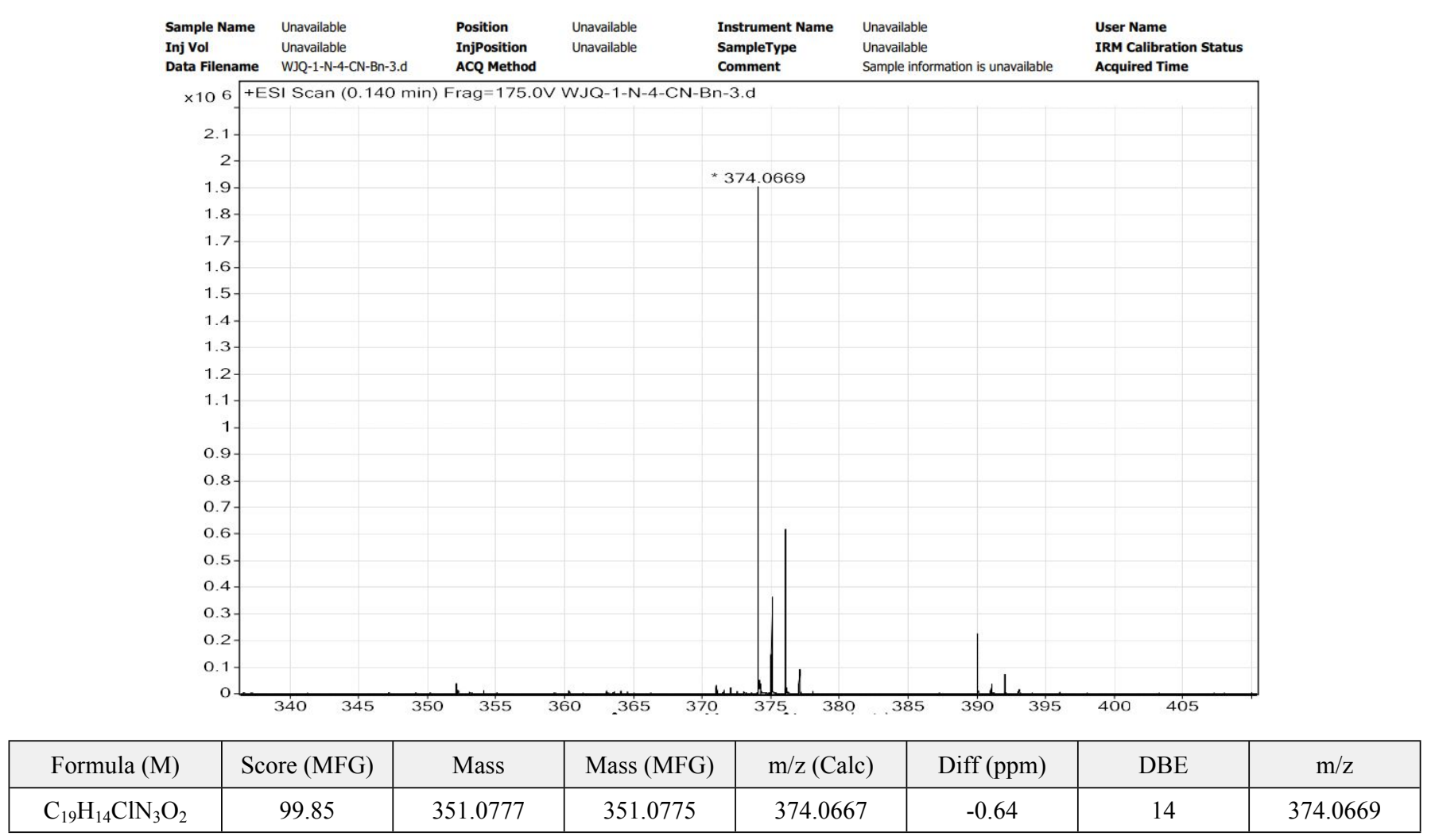

Figure S103. HRMS spectrum of $\mathbf{3 w}$.

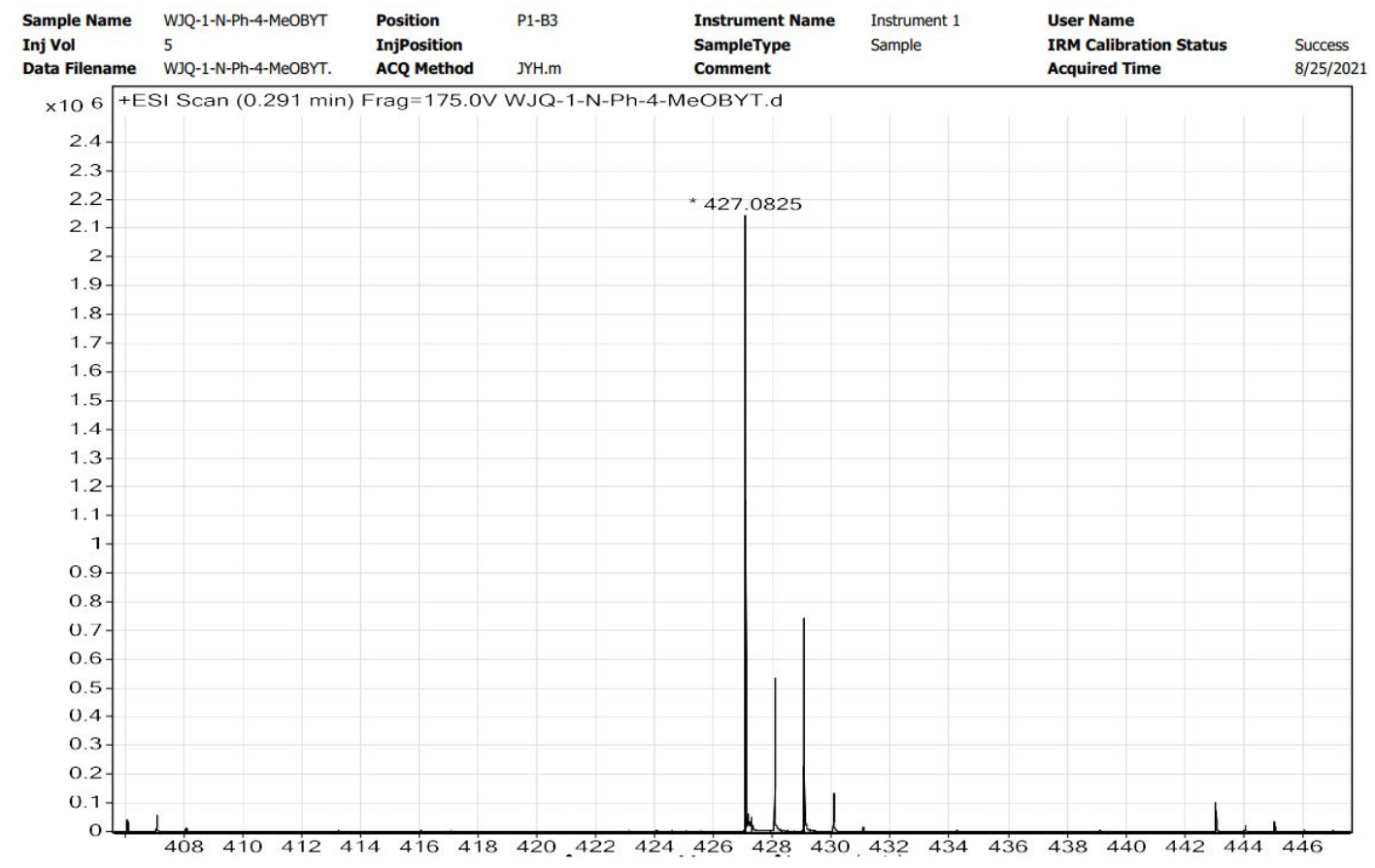

\begin{tabular}{|c|c|c|c|c|c|c|c|}
\hline Formula (M) & Score (MFG) & Mass & Mass (MFG) & $\mathrm{m} / \mathrm{z}($ Calc) & Diff (ppm) & DBE & $\mathrm{m} / \mathrm{z}$ \\
\hline $\mathrm{C}_{23} \mathrm{H}_{17} \mathrm{ClN}_{2} \mathrm{O}_{3}$ & 99.33 & 404.0933 & 404.0928 & 427.0820 & -1.26 & 16 & 427.0825 \\
\hline
\end{tabular}

Figure S104. HRMS spectrum of $\mathbf{3 x}$. 


\begin{tabular}{|c|c|c|c|c|c|c|c|}
\hline Sample Name & WJQ-1-5-Me-Ph & Position & P1-B1 & Instrument Name & Instrument 1 & $\begin{array}{l}\text { User Name } \\
\text { IRM Calibration Status }\end{array}$ & \\
\hline $\begin{array}{l}\text { Inj Vol } \\
\text { Data Filename }\end{array}$ & $\begin{array}{l}5 \\
\text { WJQ-1-5-Me-Ph-0410.d }\end{array}$ & $\begin{array}{l}\text { InjPosition } \\
\text { ACQ Method }\end{array}$ & JYH.m & $\begin{array}{l}\text { SampleType } \\
\text { Comment }\end{array}$ & & $\begin{array}{l}\text { IRM Calibration Status } \\
\text { Acquired Time }\end{array}$ & $\begin{array}{l}\text { Success } \\
\text { 4/10/2021 2:45:03 PM }\end{array}$ \\
\hline
\end{tabular}

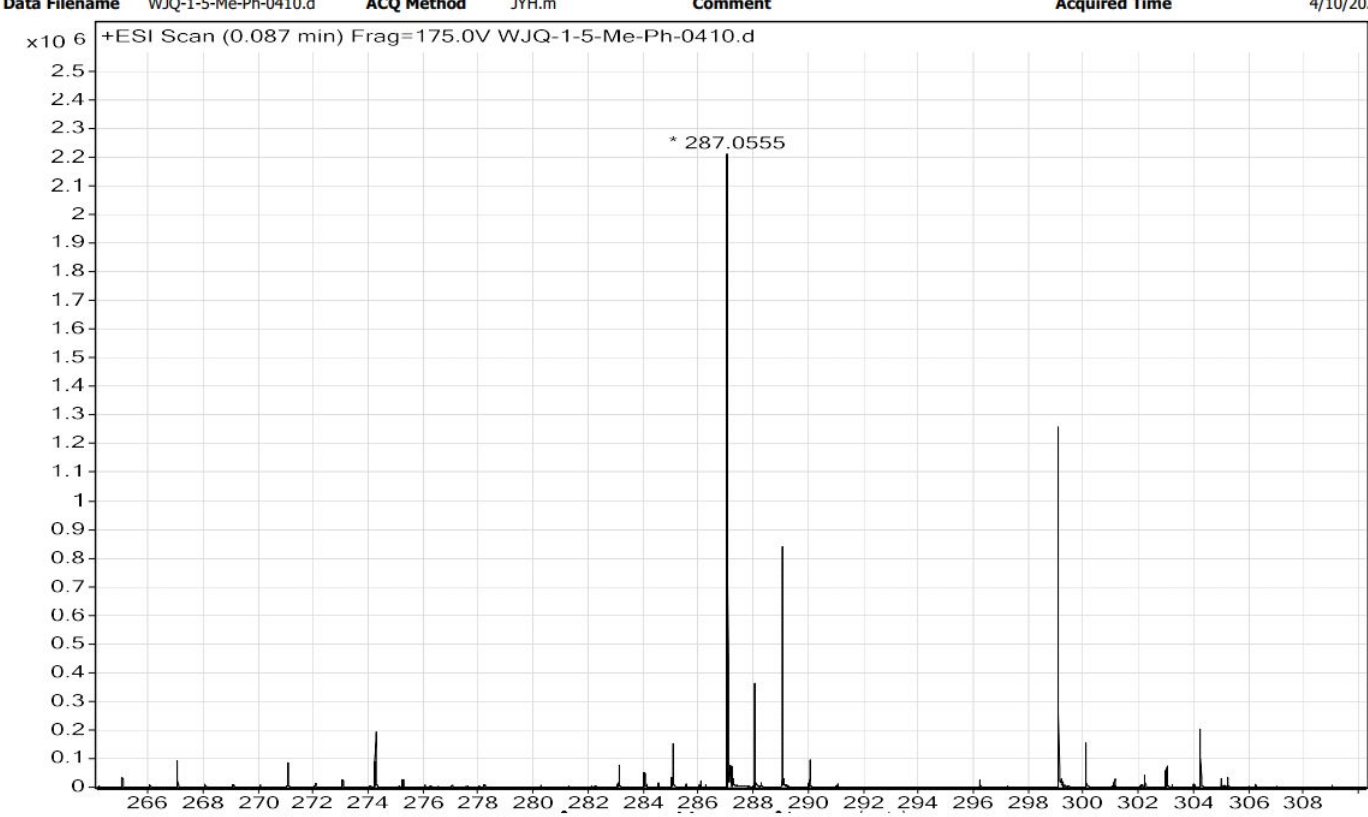

\begin{tabular}{|c|c|c|c|c|c|c|c|}
\hline Formula (M) & Score (MFG) & Mass & Mass (MFG) & $\mathrm{m} / \mathrm{z}(\mathrm{Calc})$ & Diff $(\mathrm{ppm})$ & DBE & $\mathrm{m} / \mathrm{z}$ \\
\hline $\mathrm{C}_{13} \mathrm{H}_{13} \mathrm{ClN}_{2} \mathrm{O}_{2}$ & 99.77 & 264.0663 & 264.0666 & 287.0558 & 1.05 & 8 & 287.0555 \\
\hline
\end{tabular}

Figure S105. HRMS spectrum of $\mathbf{3 y}$.

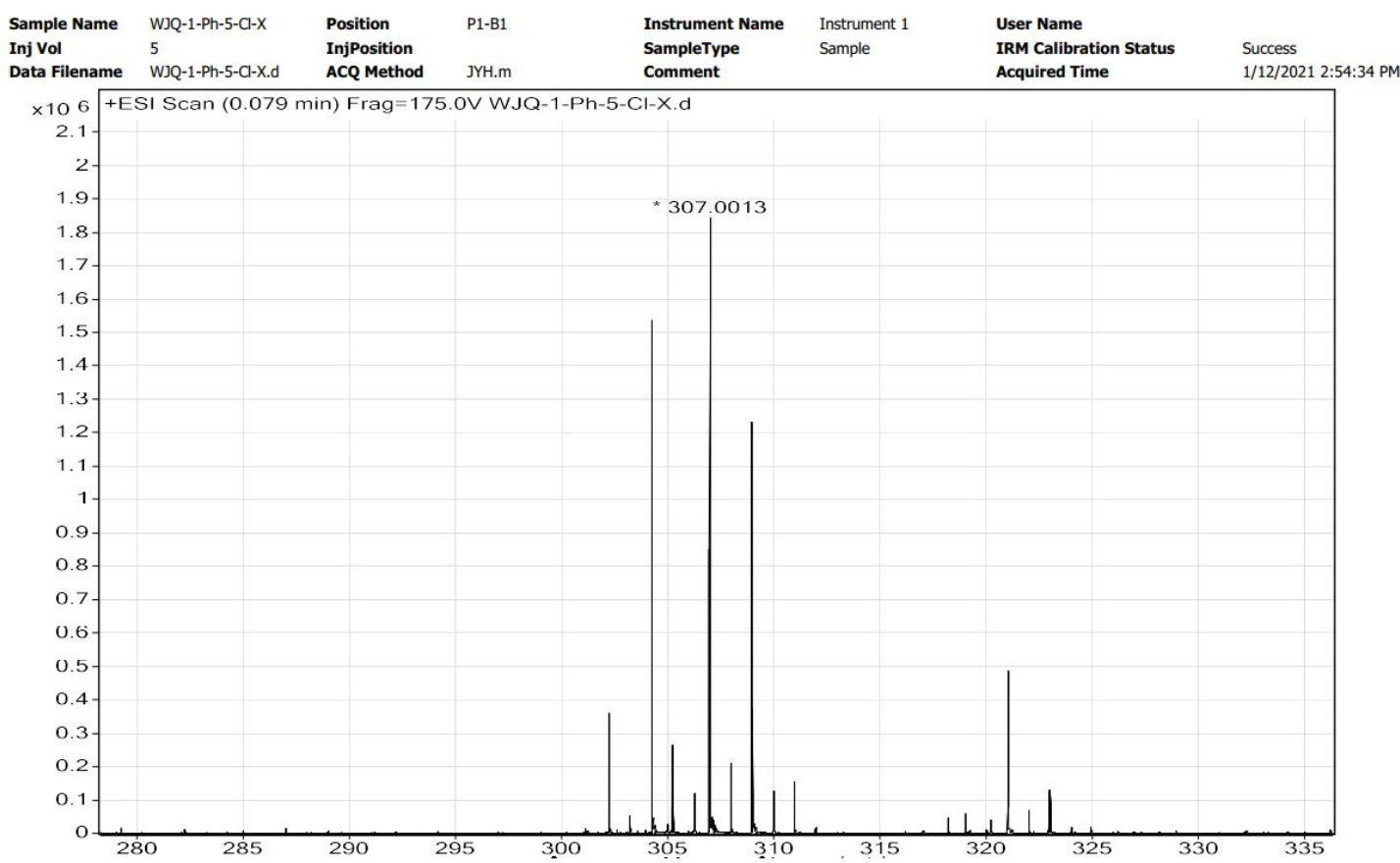

\begin{tabular}{|c|c|c|c|c|c|c|c|}
\hline Formula (M) & Score (MFG) & Mass & Mass (MFG) & $\mathrm{m} / \mathrm{z}(\mathrm{Calc})$ & Diff (ppm) & DBE & $\mathrm{m} / \mathrm{z}$ \\
\hline $\mathrm{C}_{12} \mathrm{H}_{10} \mathrm{Cl}_{2} \mathrm{~N}_{2} \mathrm{O}_{2}$ & 99.92 & 284.0121 & 284.0119 & 307.0012 & -0.51 & 8 & 307.0013 \\
\hline
\end{tabular}

Figure S106. HRMS spectrum of $\mathbf{3 z}$. 


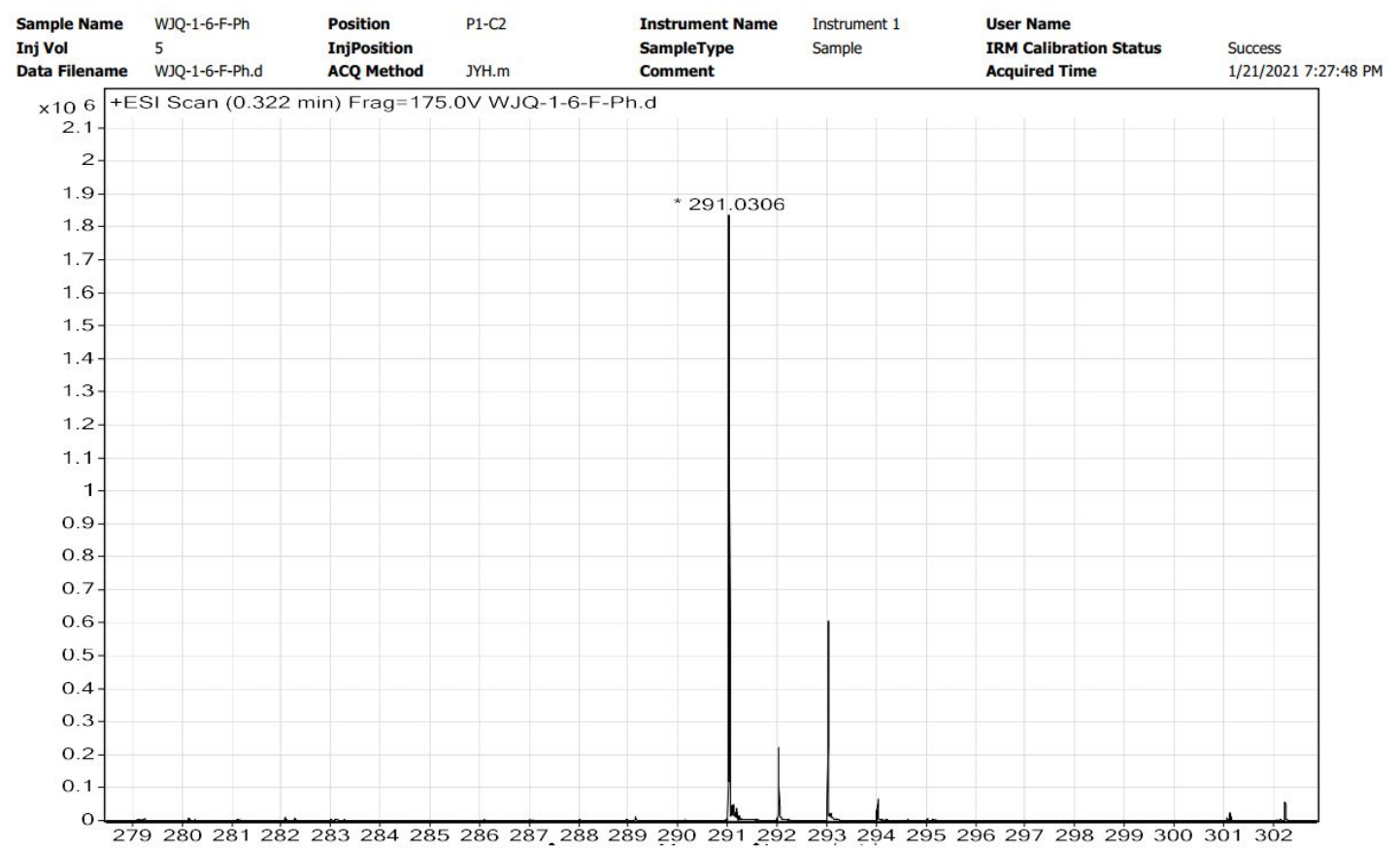

\begin{tabular}{|c|c|c|c|c|c|c|c|}
\hline Formula (M) & Score (MFG) & Mass & Mass (MFG) & $\mathrm{m} / \mathrm{z}(\mathrm{Calc})$ & Diff (ppm) & DBE & $\mathrm{m} / \mathrm{z}$ \\
\hline $\mathrm{C}_{12} \mathrm{H}_{10} \mathrm{ClFN}_{2} \mathrm{O}_{2}$ & 99.96 & 268.0414 & 268.0415 & 291.0307 & 0.39 & 8 & 291.0306 \\
\hline
\end{tabular}

Figure S107. HRMS spectrum of 3aa.

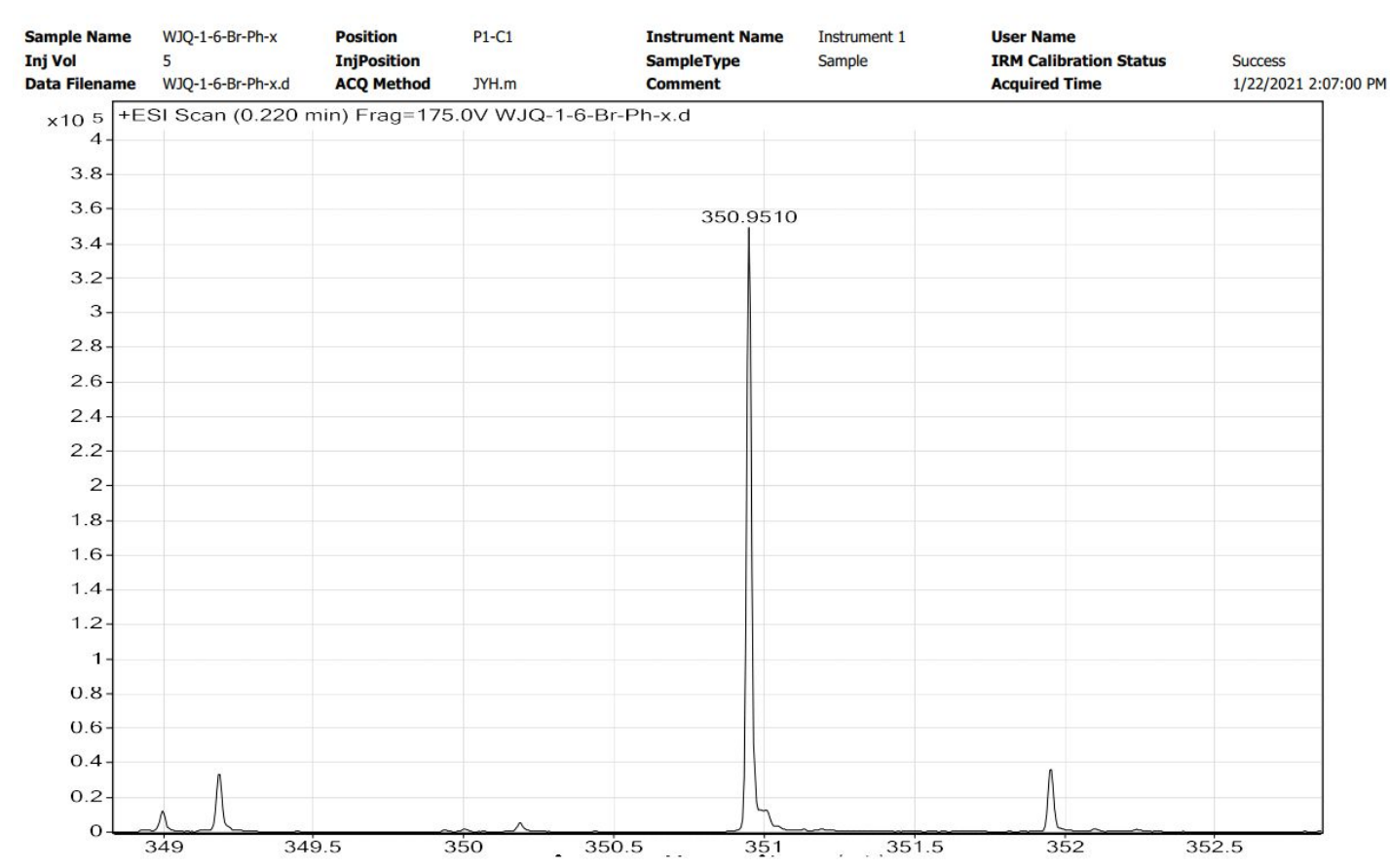

\begin{tabular}{|c|c|c|c|c|c|c|c|}
\hline Formula (M) & Score (MFG) & Mass & Mass (MFG) & $\mathrm{m} / \mathrm{z}($ Calc) & Diff (ppm) & DBE & $\mathrm{m} / \mathrm{z}$ \\
\hline $\mathrm{C}_{12} \mathrm{H}_{10} \mathrm{BrClN}_{2} \mathrm{O}_{2}$ & 99.58 & 327.9618 & 327.9614 & 350.9506 & -1.1 & 8 & 350.9510 \\
\hline
\end{tabular}

Figure S108. HRMS spectrum of 3ab. 


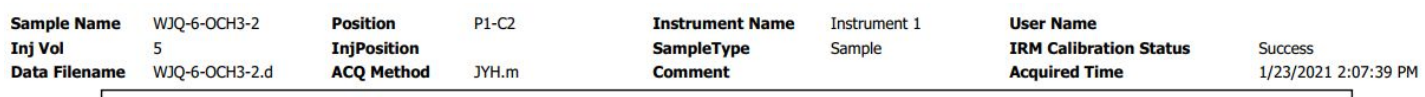

x10 5 +ESI Scan (0.185 min) Frag=175.0V WJQ-6-OCH3-2.d

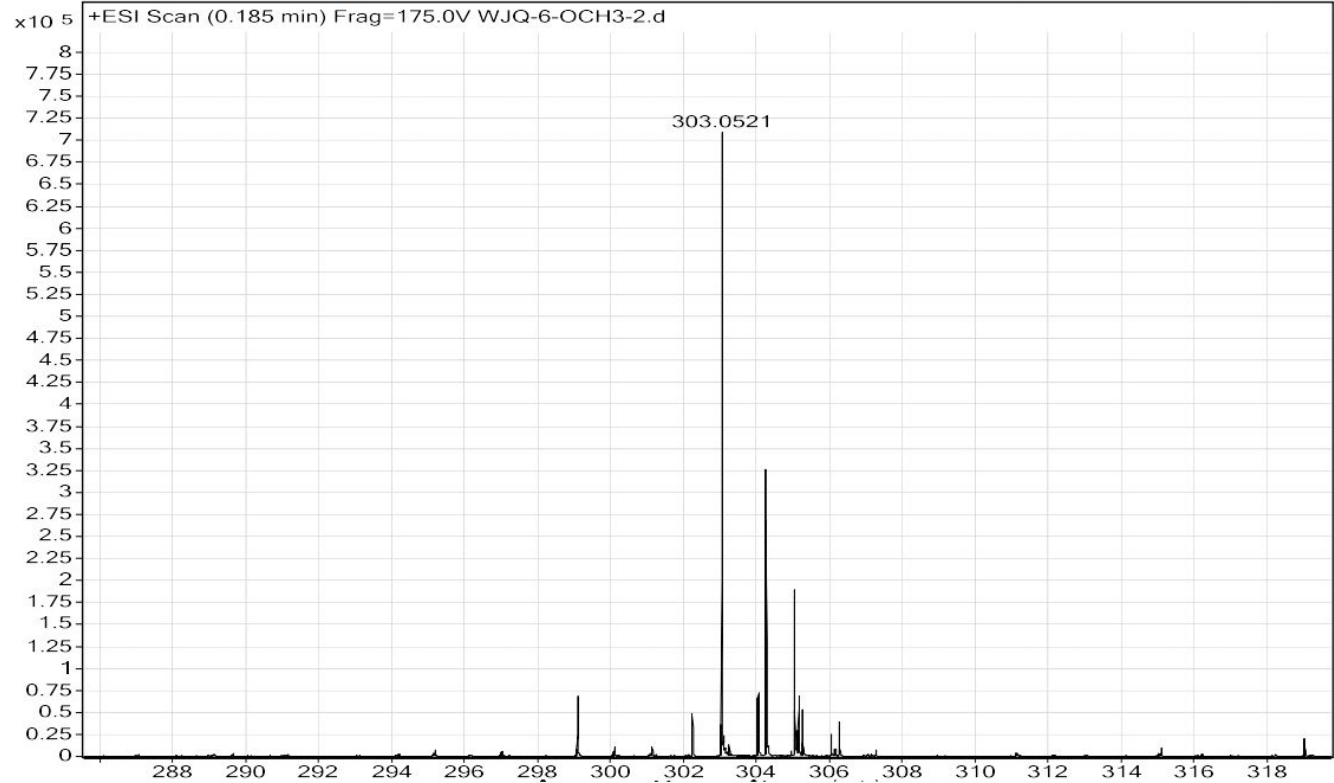

\begin{tabular}{|c|c|c|c|c|c|c|c|}
\hline Formula (M) & Score (MFG) & Mass & Mass (MFG) & $\mathrm{m} / \mathrm{z}($ Calc) & Diff (ppm) & DBE & $\mathrm{m} / \mathrm{z}$ \\
\hline $\mathrm{C}_{13} \mathrm{H}_{13} \mathrm{ClN}_{2} \mathrm{O}_{3}$ & 93.13 & 280.0629 & 280.0615 & 303.0507 & -5.03 & 8 & 303.0521 \\
\hline
\end{tabular}

Figure S109. HRMS spectrum of 3ac.

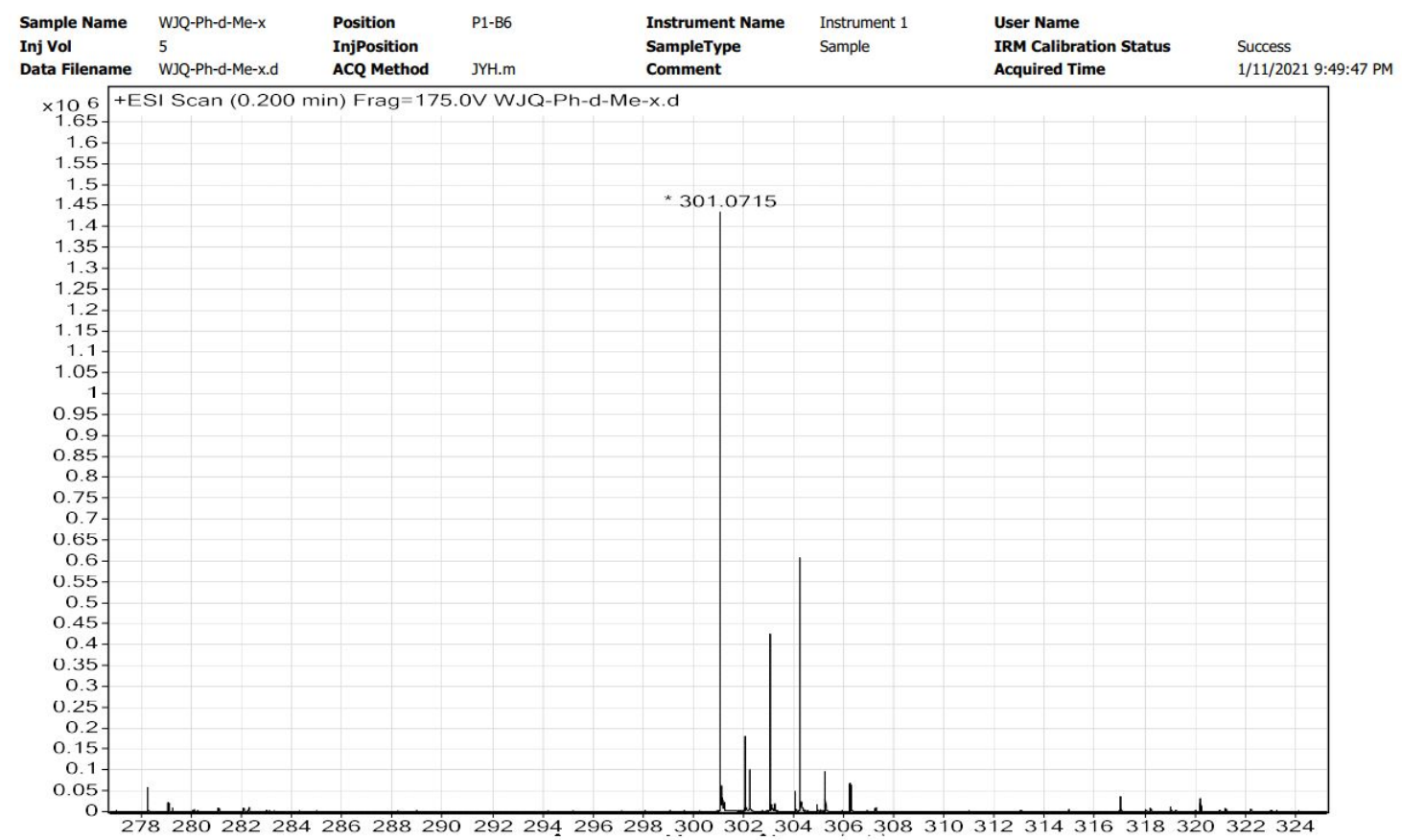

\begin{tabular}{|c|c|c|c|c|c|c|c|}
\hline Formula (M) & Score (MFG) & Mass & Mass (MFG) & $\mathrm{m} / \mathrm{z}(\mathrm{Calc})$ & Diff $(\mathrm{ppm})$ & DBE & $\mathrm{m} / \mathrm{z}$ \\
\hline $\mathrm{C}_{14} \mathrm{H}_{15} \mathrm{ClN}_{2} \mathrm{O}_{2}$ & 99.98 & 278.0823 & 278.0822 & 301.0714 & -0.26 & 8 & 301.0715 \\
\hline
\end{tabular}

Figure S110. HRMS spectrum of $\mathbf{3 a d}$. 


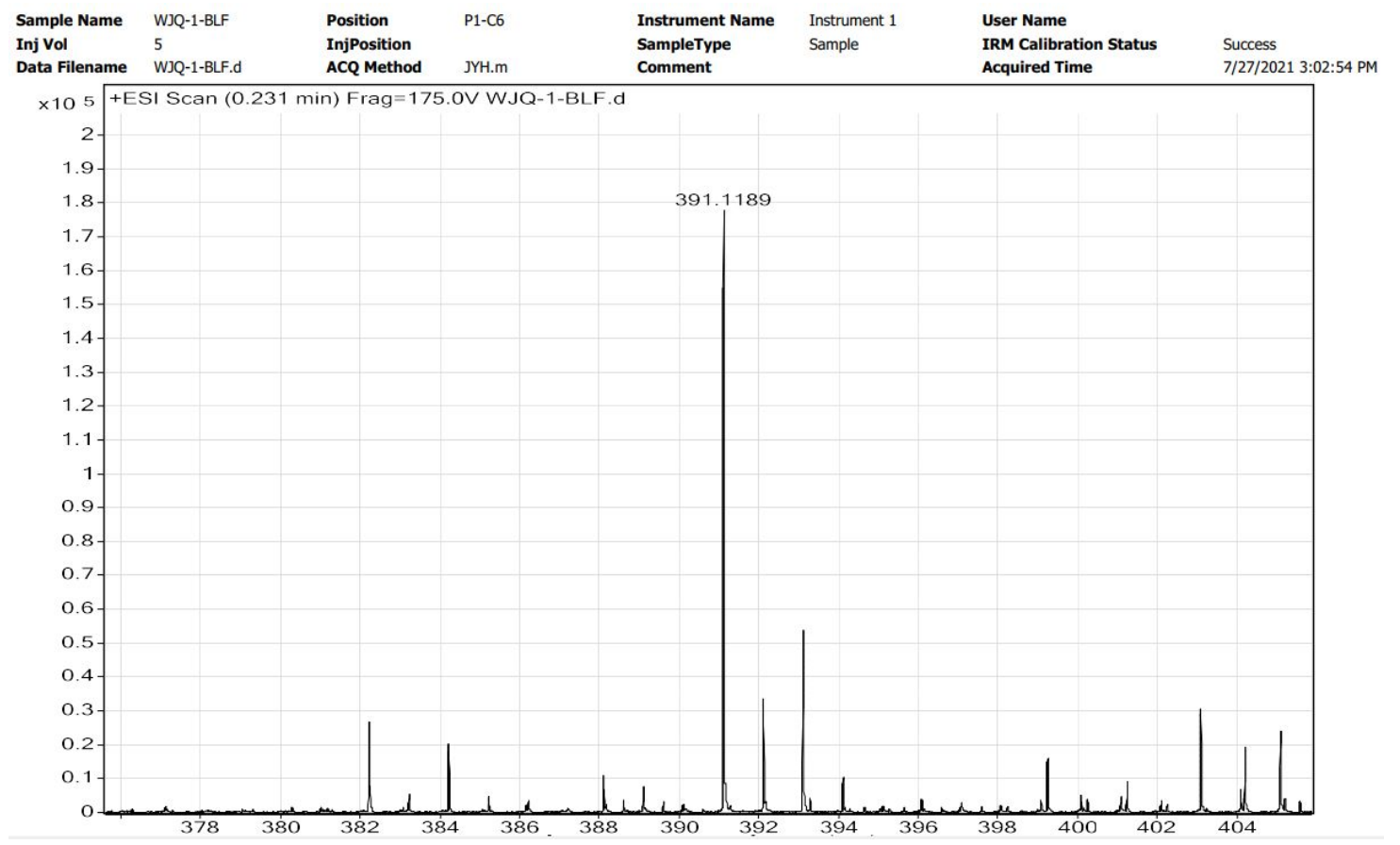

\begin{tabular}{|c|c|c|c|c|c|c|c|}
\hline Formula (M) & Score (MFG) & Mass & Mass (MFG) & $\mathrm{m} / \mathrm{z}($ Calc) & Diff (ppm) & DBE & $\mathrm{m} / \mathbf{z}$ \\
\hline $\mathrm{C}_{21} \mathrm{H}_{21} \mathrm{ClN}_{2} \mathrm{O}_{2}$ & 99.22 & 368.1297 & 368.1292 & 391.1184 & -1.42 & 12 & 391.1189 \\
\hline
\end{tabular}

Figure S111. HRMS spectrum of 3ae.

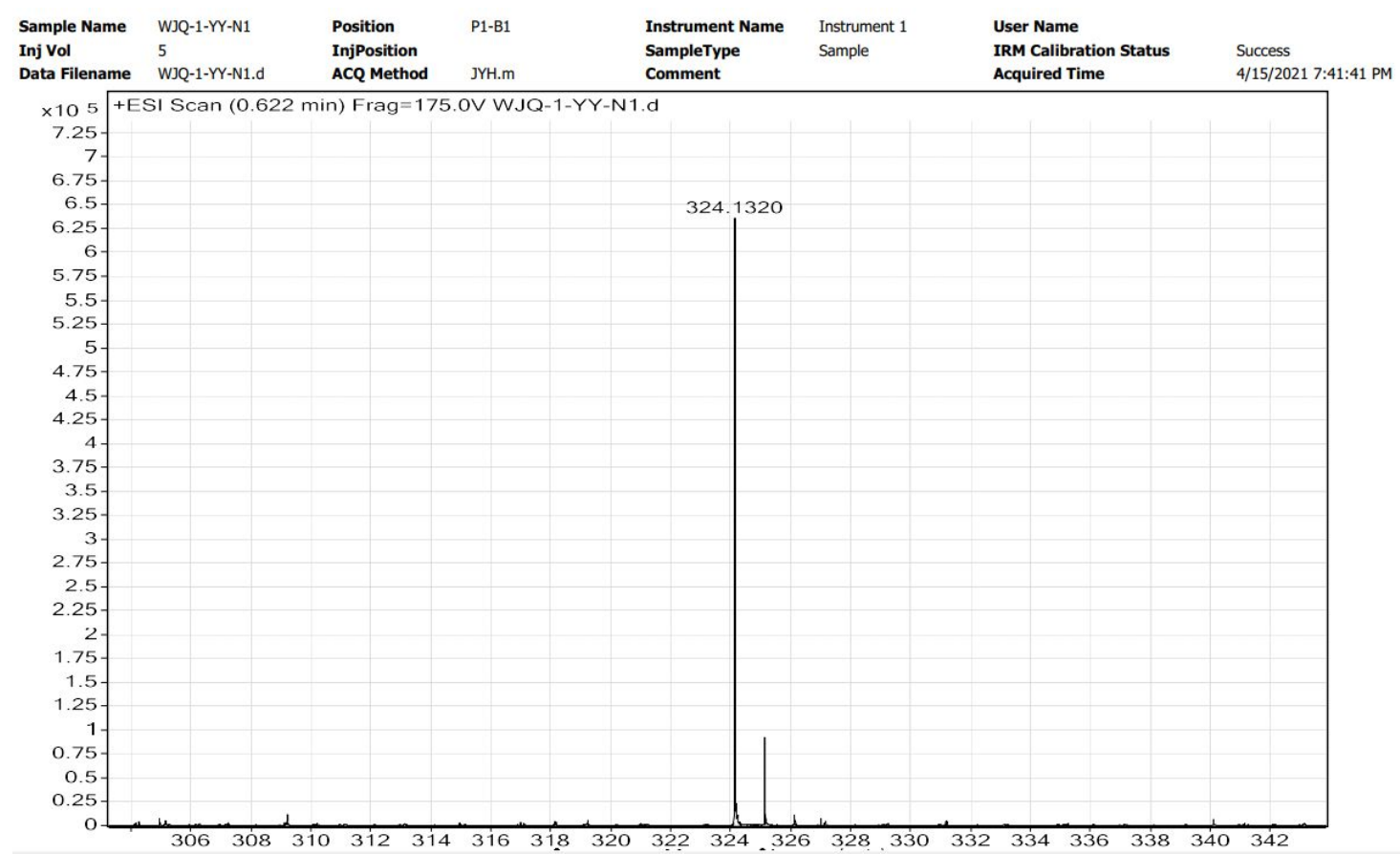

\begin{tabular}{|c|c|c|c|c|c|c|c|}
\hline Formula (M) & Score (MFG) & Mass & Mass (MFG) & $\mathrm{m} / \mathrm{z}($ Calc) & Diff $(\mathrm{ppm})$ & DBE & $\mathrm{m} / \mathrm{z}$ \\
\hline $\mathrm{C}_{16} \mathrm{H}_{19} \mathrm{~N}_{3} \mathrm{O}_{3}$ & 99.93 & 301.1428 & 301.1426 & 324.1319 & -0.46 & 9 & 324.132 \\
\hline
\end{tabular}

Figure S112. HRMS spectrum of $\mathbf{4 a}$. 


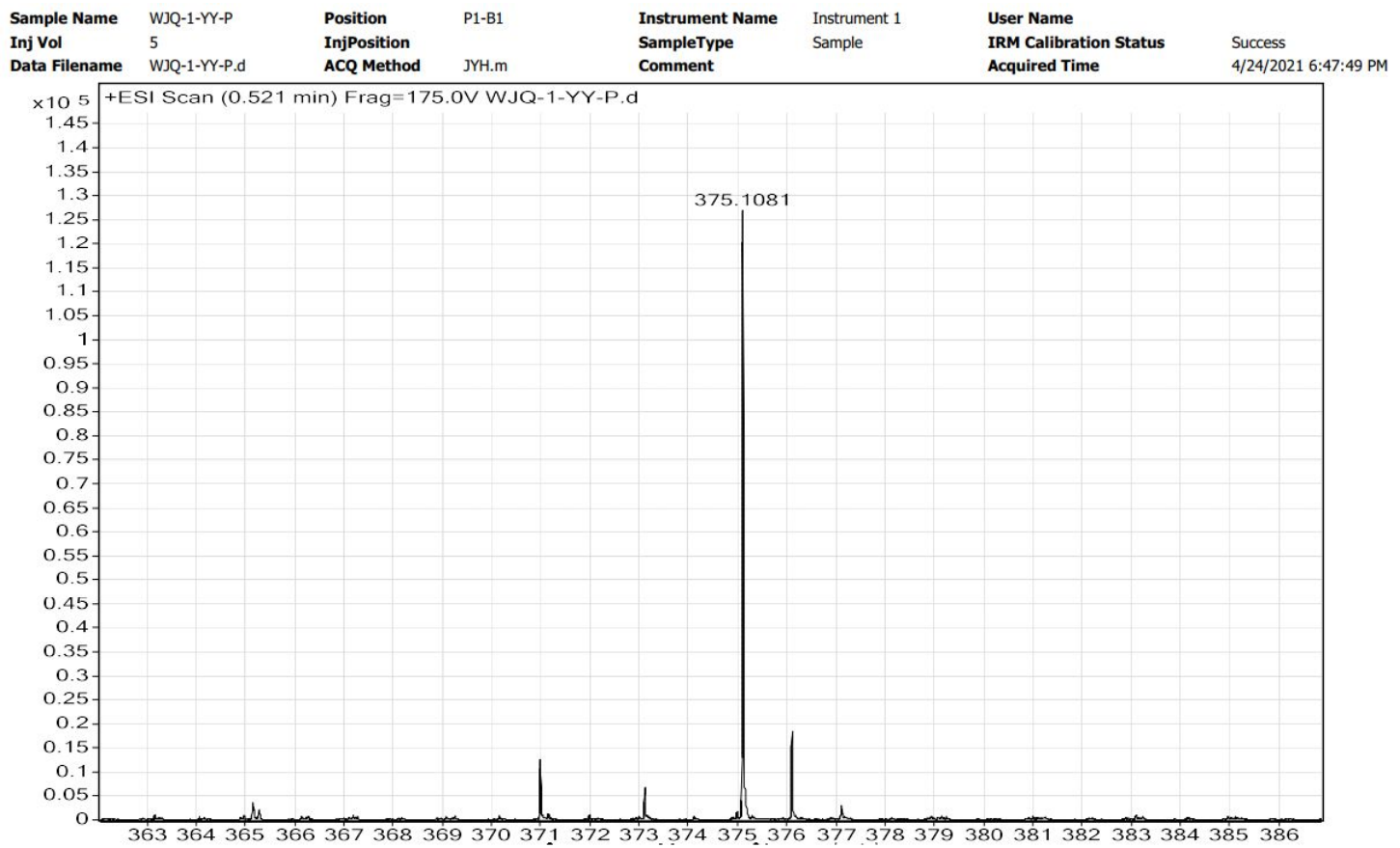

\begin{tabular}{|c|c|c|c|c|c|c|c|}
\hline Formula (M) & Score (MFG) & Mass (MFG) & Mass & $\mathrm{m} / \mathrm{z}(\mathrm{Calc})$ & Diff $(\mathrm{ppm})$ & DBE & $\mathrm{m} / \mathrm{z}$ \\
\hline $\mathrm{C}_{16} \mathrm{H}_{21} \mathrm{~N}_{2} \mathrm{O}_{5} \mathrm{P}$ & 99.98 & 352.1188 & 352.1189 & 375.1080 & -0.2 & 8 & 375.1081 \\
\hline
\end{tabular}

Figure S113. HRMS spectrum of $\mathbf{4 b}$.

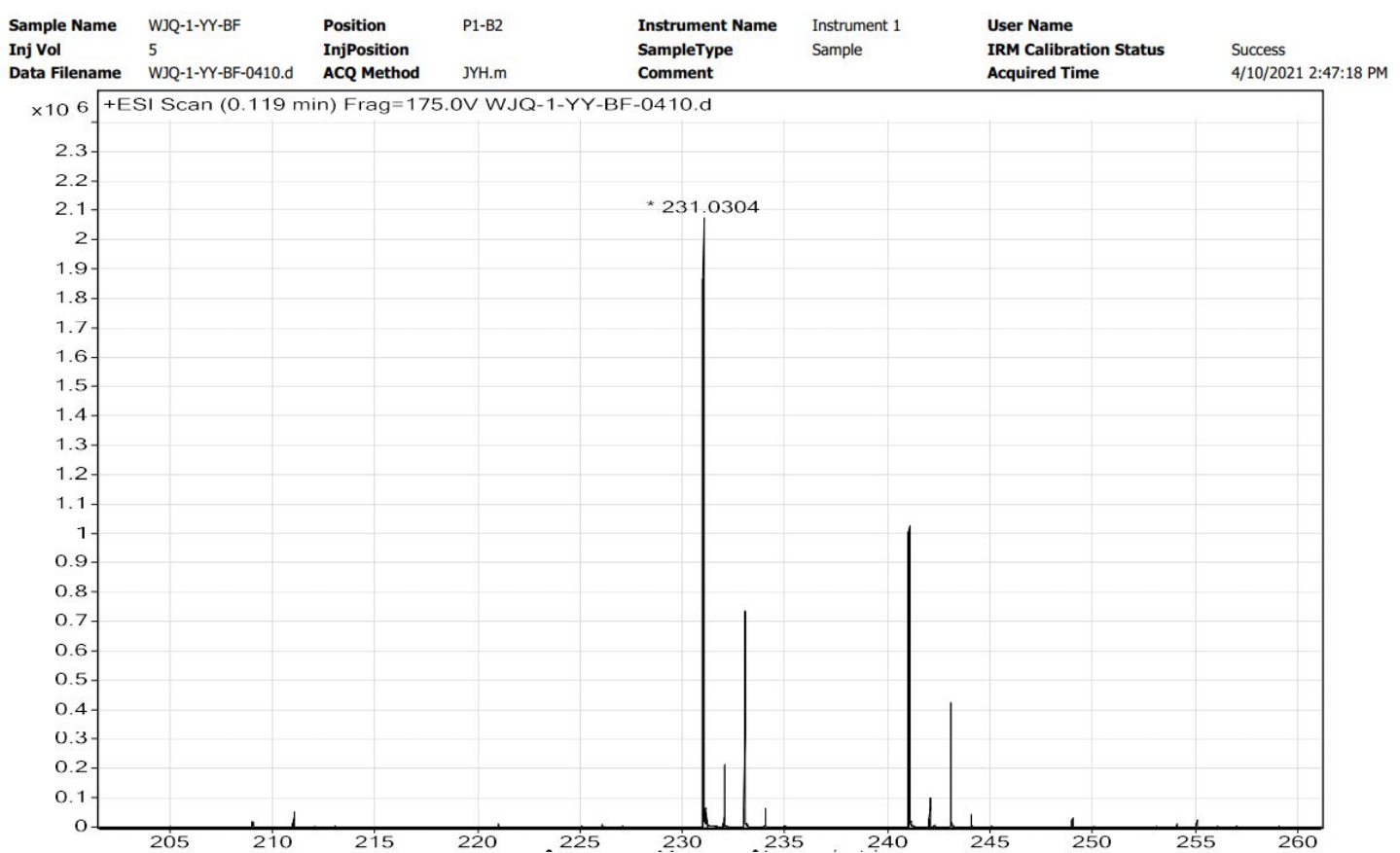

\begin{tabular}{|c|c|c|c|c|c|c|c|}
\hline Formula (M) & Score (MFG) & Mass & Mass (MFG) & $\mathrm{m} / \mathrm{z}($ Calc) & Diff (ppm) & DBE & $\mathrm{m} / \mathrm{z}$ \\
\hline $\mathrm{C}_{10} \mathrm{H}_{9} \mathrm{ClN}_{2} \mathrm{O}$ & 96.77 & 208.0412 & 208.0403 & 231.0296 & -4.03 & 7 & 231.0304 \\
\hline
\end{tabular}

Figure S114. HRMS spectrum of 4c. 


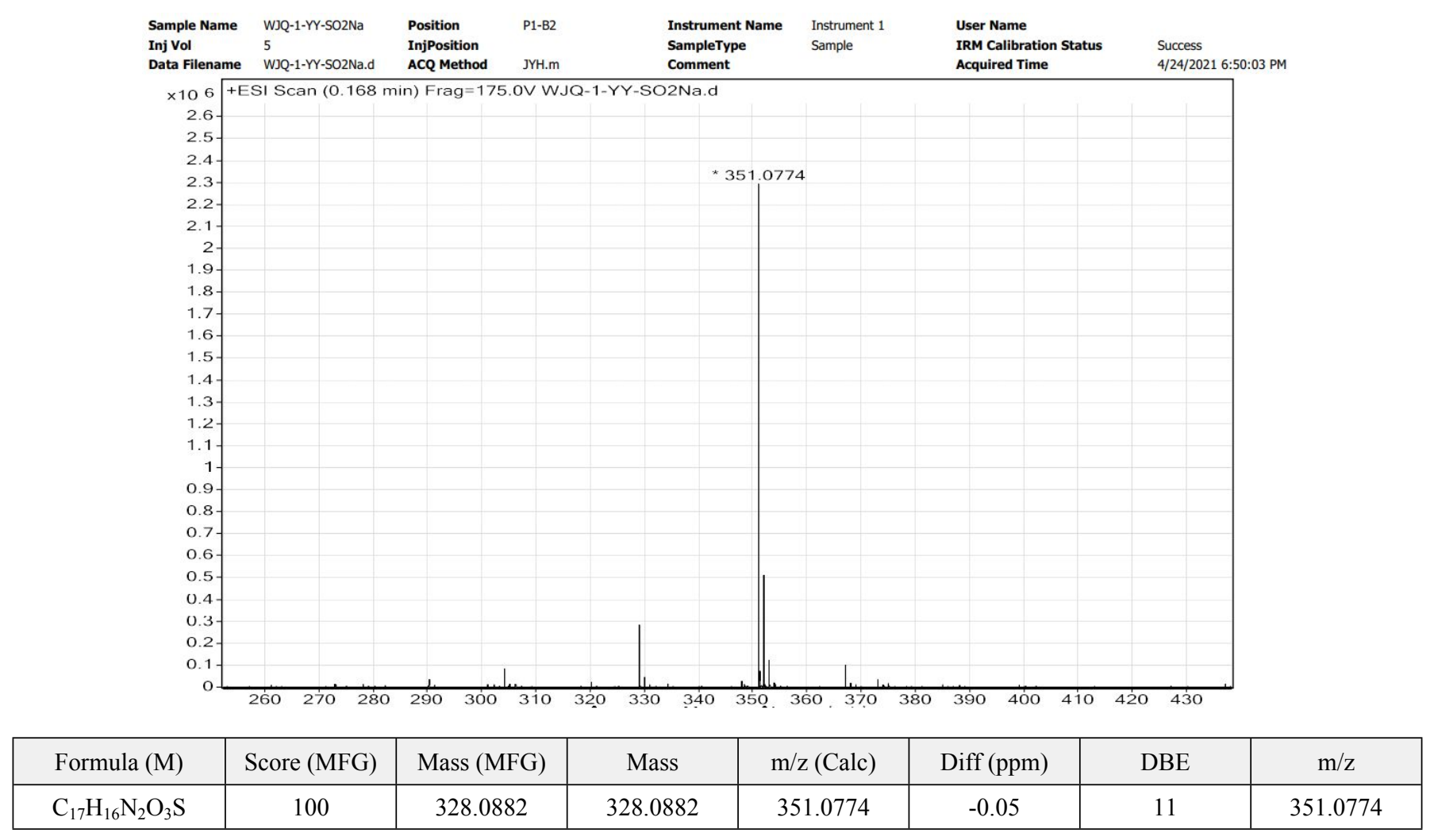

Figure S115. HRMS spectrum of 4d. 